Supporting Information

\title{
A Domino Route toward Polysubstituted Pyrroles from 2-Imidazolines and Electron-Deficient Alkynes
}

Nikita E. Golantsov,* Alexandra S. Golubenkova, Alexey A. Festa, Alexey V. Varlamov, Leonid G. Voskressensky*

Organic Chemistry Department, Science Faculty, Peoples' Friendship University of Russia (RUDN University), 6 Miklukho-Maklaya St., Moscow 117198, Russia

\section{Corresponding Authors}

Email: golantsov-ne@rudn.ru, lvoskressensky@ sci.pfu.edu.ru 


\section{Content}

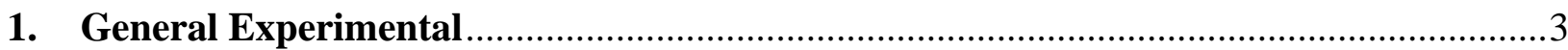

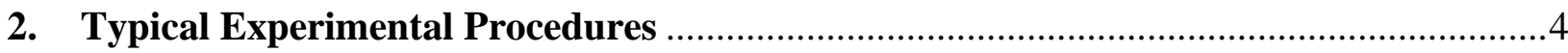

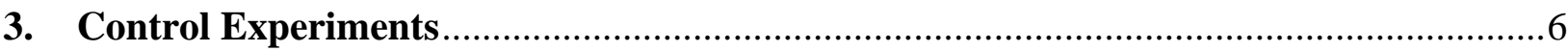

3.1 Procedure for the microwave-assisted synthesis of pyrrole 4a and pyrrolopyrazine 5 ....

3.2 Procedure for the detecting non-aromatic intermediate 7 and its transformation into pyrrole 4a and pyrrolopyrazine 5 7

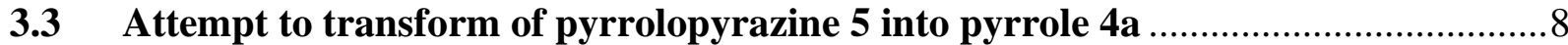

3.4 Procedure for the thermal synthesis of pyrrolopyrazine 5 from imidazolidine $3 a$...8

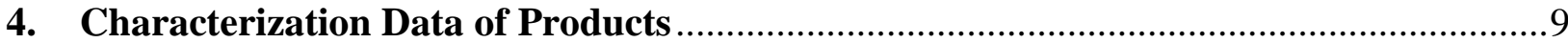

4.1 $\quad N$-aryl-2-imidazolines and 1-methyl-2-phenyl-2-imidazoline …............................

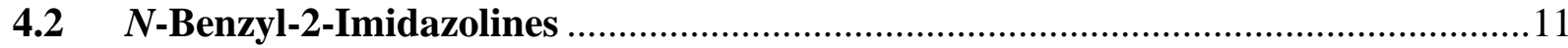

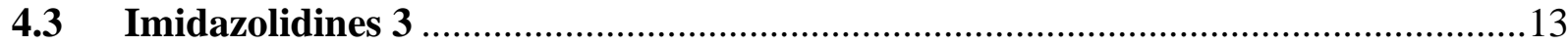

4.4 Dimethyl 2-benzoyl-1-[2-(arylamino)ethyl]-1H-pyrrole-3,4-dicarboxylates 4:......22

4.5 Dimethyl 2-benzoyl-1-[2-(arylamino)ethyl]-1 $H$-pyrrole-3,4-dicarboxylates ..........26

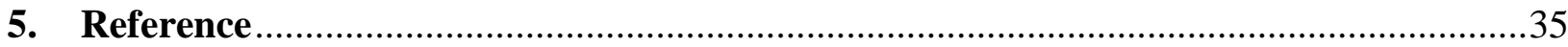

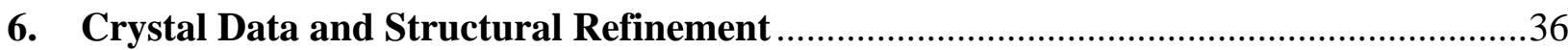

6.1 Crystal data and structural refinement for imidazolidine $\mathbf{3 g}$....................................36

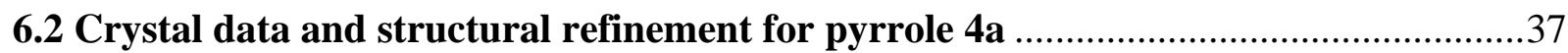

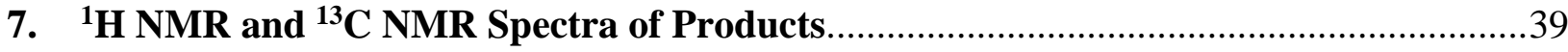




\section{General Experimental}

NMR measurements were performed on Jeol JNM-ECA 600 spectrometer with operating frequencies of $600 \mathrm{MHz}$ and $150 \mathrm{MHz}$, respectively, at room temperature. The chemical shifts are reported in parts per million (ppm) relative to the solvent residual peak of DMSO-d6 $\left({ }^{1} \mathrm{H}: 2.50\right.$ ppm, ${ }^{13} \mathrm{C}$ : $39.52 \mathrm{ppm}$, septet), $\mathrm{CDCl}_{3}\left({ }^{1} \mathrm{H}: 7.26 \mathrm{ppm}\right.$, singlet, ${ }^{13} \mathrm{C}: 77.16 \mathrm{ppm}$, triplet $), \mathrm{C}_{6} \mathrm{D}_{6}\left({ }^{1} \mathrm{H}\right.$ : $7.16 \mathrm{ppm},{ }^{13} \mathrm{C}$ : $128.06 \mathrm{ppm}$, triplet). The following abbreviations were used to explain multiplicities: $\mathrm{s}=$ singlet, $\mathrm{d}=$ doublet, $\mathrm{t}=$ triplet, $\mathrm{q}=$ quartet, $\mathrm{dd}=$ doublet of doublets, $\mathrm{m}=$ multiplet, br $=$ broad singlet.

LC-ESI measurements were carried out on LCMS-8040 Triple quadrupole liquid chromatograph mass spectrometer (Shimadzu).

HRMS measurements were carried out on a MicrOTOF-Q II system (Bruker).

IR spectra were recorded on an Infralum FT-801 FT/IR instrument.

TLC was performed using Sorbfil silica plates with short-wave UV light (254 nm) as visualizing agent.

Melting points were determined on a SMP-10 apparatus and were uncorrected.

Preparative column chromatography purification was performed using silica gel (60-75 mesh). Microwave irradiation experiments were carried out in a dedicated Anton Paar Monowave 400 microwave apparatus, operating at a frequency of $2.45 \mathrm{GHz}$ with continuous irradiation power from 0 to $300 \mathrm{~W}$ with utilization of the standard absorbance level of $300 \mathrm{~W}$ maximum power. The reactions were carried out in 10- or 30-mL glass tubes, sealed with Teflon septum and placed in the microwave cavity. The reaction mixture was irradiated at a required ceiling temperature using maximum power for the stipulated time. Then it was cooled to ambient temperature with gas jet cooling.

All other reagents for synthesis were purchased from commercial sources and were used without further purification or were prepared according to standard literature procedures.

Solvents were distilled and dried according to standard methods prior to use. 


\section{Typical Experimental Procedures}

\subsection{General procedure for the synthesis of $N$-aryl-2-imidazolines and 1-methyl-2-phenyl-2- imidazoline 1 (GP1)}

$\mathrm{N}$-Aryl-2-imidazolines and $\mathrm{N}$-methyl-2-imidazoline were prepared according to a previously described procedure ${ }^{[1]}$.

$\mathrm{N}$-Benzoyl ethanolamine $(25 \mathrm{mmol})$ reacted with thionyl chloride $(7.3 \mathrm{~mL}, 0.1 \mathrm{~mol})$ at reflux for $4 \mathrm{~h}$ using magnetic stirrer Hei-Standard (Heidolph) as the heat source. Excess thionyl chloride was evaporated in vacuo. The residue was dissolved in dry diethyl ether $(50 \mathrm{~mL})$ and filtered to remove insoluble impurities. To this solution was added dry triethylamine $(12.5 \mathrm{~mL}, 75 \mathrm{mmol})$, followed by corresponding amine ( $28 \mathrm{mmol})$. After stirring overnight at room temperature $10 \% \mathrm{NaOH}(100$ $\mathrm{mL})$ was added and stirred for $24 \mathrm{~h}$. The aqueous phase was extracted with AcOEt $(50 \mathrm{~mL} \times 3)$ and the combined organic layer was dried over anhydrous $\mathrm{Na}_{2} \mathrm{SO}_{4}$ and evaporated in vacuo. The crude product was purified by preparative chromatography on $\mathrm{SiO}_{2}$ to afford corresponding imidazolines.

Most of the prepared $N$-arylimidazolines and $N$-methyl-2-imidazoline are known and fully characterized. 2-Phenyl-1-[4-(trifluoromethyl)phenyl]-4,5-dihydro-1H-imidazole (1f) is new compound (vide infra).

\subsection{General procedure for the synthesis of $N$-benzyl-2-imidazolines 1 (GP2)}

$\mathrm{N}$-Benzyl-2-imidazolines were prepared according to a previously described procedure by reacting $\mathrm{N}$-benzylethylenediamine with the corresponding benzaldehyde in the presence of NBS. ${ }^{[2]}$

The mixture of aldehyde $(38 \mathrm{mmol})$ and $\mathrm{N}$-benzylethelenediamine $(40 \mathrm{mmol})$ in dry $\mathrm{CH}_{2} \mathrm{Cl}_{2}(125$ $\mathrm{ml}$ ) was stirred at $0^{\circ} \mathrm{C}$ for $30 \mathrm{~min}$, NBS (40 mmol) was added to the mixture and the resulting solution was stirred overnight at rt. Reaction was diluted by $\mathrm{CH}_{2} \mathrm{Cl}_{2}(125 \mathrm{~mL})$ and quenched by the addition of mixture of $\mathrm{Na}_{2} \mathrm{~S}_{2} \mathrm{O}_{5}$ aq $(140 \mathrm{~mL})$ and $10 \% \mathrm{NaOH}$ aq $(70 \mathrm{~mL})$, then organic layer was washed by $10 \% \mathrm{NaOH}$ aq $(70 \mathrm{~mL})$, dried over anhydrous $\mathrm{Na}_{2} \mathrm{SO}_{4}$ and evaporated in vacuo. The residue was purified by $\mathrm{SiO}_{2}$ column chromatography. 1-Benzyl-2-phenyl-4,5-dihydro- $1 H$ imidazole (1b) and 1-benzyl-2-(2-thienyl)-4,5-dihydro-1H-imidazole (11) are known and fully characterized. The majority of the prepared imidazolines have not been reported before (vide infra).

\subsection{General procedure for the synthesis of imidazolidines 3 (GP3)}

Methyl propiolate or but-3-yn-2-one $(3.55 \mathrm{mmol})$ was added to the solution of the corresponding imidazoline 1a-l $(1.7 \mathrm{mmol})$ in dry acetonitrile or toluene $(8 \mathrm{~mL})$. The reaction was monitored by 
TLC. After completion of the reaction, the solvent was evaporated in vacuo. The crude product was purified by chromatography on $\mathrm{SiO}_{2}$ to afford imidazolidines 3a-r.

2.4 General procedure for the synthesis of the dimethyl 2-benzoyl-1-[2-(arylamino)ethyl]$1 H$-pyrrole-3,4-dicarboxylates 4 (GP4)

A solution of corresponding imidazolidine $3(0.5 \mathrm{mmol})$ in $o$-xylene $(10 \mathrm{~mL})$ was refluxed for 0.5 $\mathrm{h}$ under vigorous stirring in the air using magnetic stirrer Hei-Standard (Heidolph) as the heat source. After removal of $o$-xylene in vacuo, the crude oily pyrroles were purified by column chromatography.

\subsection{One pot procedure for the synthesis of dimethyl 2-benzoyl-1-[2-(arylamino)ethyl]-1H- pyrrole-3,4-dicarboxylates 4 (GP5)}

Methyl propiolate or but-3-yn-2-one $(2.31 \mathrm{mmol})$ was added to the solution of the corresponding imidazoline $1(1.1 \mathrm{mmol})$ in dry $o$-xylene $(15 \mathrm{~mL})$. The reaction progress was monitored by TLC. After consumption of imidazoline, the reaction mixture was refluxed in an open vessel for 30 min using magnetic stirrer Hei-Standard (Heidolph) as the heat source. After removal of $o$-xylene in vacuo, the crude oily pyrroles were purified by column chromatography.

\subsection{General procedure for the synthesis of the dimethyl 2-benzoyl-1-[2-(arylamino)ethyl] -1H-pyrrole-3,4-dicarboxylates 6 (GP6)}

A mixture of imidazolidine $3(1 \mathrm{mmol})$ and corresponding acylating agent $(1.1 \mathrm{mmol})$ was refluxed in an open vessel in $o$-xylene (12 mL) for 30 min using magnetic stirrer Hei-Standard (Heidolph) as the heat source. After removal of $o$-xylene in vacuo, the crude oily pyrroles were purified by column chromatography.

\subsection{One pot procedure for the pseudo-five-component synthesis of the dimethyl 2- benzoyl-1-[2-(arylamino)ethyl] -1H-pyrrole-3,4-dicarboxylates 6 (GP7)}

Methyl propiolate or but-3-yn-2-one $(2.31 \mathrm{mmol})$ was added to the solution of the corresponding imidazoline $1(1.1 \mathrm{mmol})$ in dry $o$-xylene $(12 \mathrm{~mL})$. The reaction progress was monitored by TLC. After consumption of imidazoline, the corresponding acylating agent $(1.1 \mathrm{mmol})$ was added and the reaction mixture was refluxed in an open vessel for 30 min using magnetic stirrer Hei-Standard (Heidolph) as the heat source. After removal of o-xylene in vacuo, the crude oily pyrroles were purified by column chromatography. 


\section{Control Experiments}

\subsection{Procedure for the microwave-assisted synthesis of pyrrole $4 a$ and pyrrolopyrazine 5}

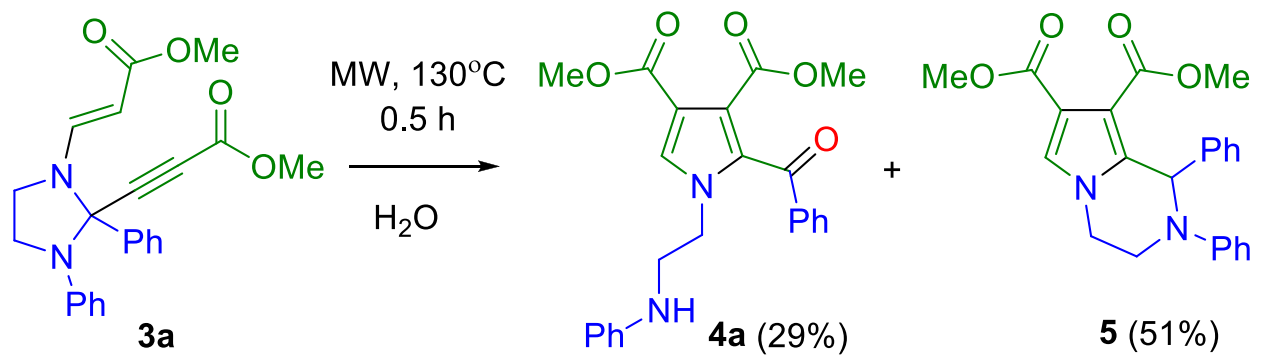

Under argon a solution of methyl (2E)-3-[2-(3-methoxy-3-oxoprop-1-yn-1-yl)-2,3diphenylimidazolidin-1-yl]prop-2-enoate 3a (195 mg, $0.5 \mathrm{mmol})$ in water (2 mL) was irradiated in 10-mL glass vessel sealed with Teflon septum in microwave reactor Monowave 300 (Anton Paar $\mathrm{GmbH}$ ) at temperature $130^{\circ} \mathrm{C}$ for $30 \mathrm{~min}$. Then it was cooled to ambient temperature with gas jet cooling, then the glass vessel was opened and left to stay on the air at room temperature for 12 h. After removal of water in vacuo, the residue was purified by column chromatography with chloroform: hexane (1:1) as eluent yielded pyrrole $\mathbf{4 a}(59 \mathrm{mg}, 29 \%)$ and pyrrolo[1,2-a]pyrazine 5 (100mg, 51\%).

\section{Dimethyl 1,2-diphenyl-1,2,3,4-tetrahydropyrrolo[1,2-a]pyrazine-7,8-dicarboxylate (5)}<smiles>CC(=O)c1cn2c(c1C(C)=O)C(c1ccccc1)N(c1ccccc1)CC2</smiles>

${ }^{1} \mathrm{H} \mathrm{NMR}\left(\mathrm{CDCl}_{3}, 600 \mathrm{MHz}\right): \delta 3.58$ - $3.65(\mathrm{~m}, 1 \mathrm{H}), 3.66$ - $3.71(\mathrm{~m}, 1$ H), $3.72(\mathrm{~s}, 3 \mathrm{H}), 3.83$ (s, $3 \mathrm{H}), 3.88$ (dt, 1H, J=12.6, $4.3 \mathrm{~Hz}$ ), 4.05 (ddd, $1 \mathrm{H}, J=12.6,9.1,5.0 \mathrm{~Hz}), 6.54(\mathrm{~s}, 1 \mathrm{H}), 6.88(\mathrm{t}, 1 \mathrm{H}, J=7.3 \mathrm{~Hz}), 6.96(\mathrm{~d}$, $2 \mathrm{H}, J=8.1 \mathrm{~Hz}), 7.16(\mathrm{~d}, 2 \mathrm{H}, J=7.1 \mathrm{~Hz}), 7.18(\mathrm{~s}, 1 \mathrm{H}), 7.21-7.30(\mathrm{~m}, 5$ H) ppm. ${ }^{13} \mathrm{C}\{1 \mathrm{H}\} \mathrm{NMR}\left(\mathrm{CDCl}_{3}, 150 \mathrm{MHz}\right): \delta 42.3,42.7,51.5,51.7,57.4,111.9,115.9,116.1$ (2C), 120.0, 126.0, 127.5 (2C), 127.6, 128.4 (2C), 129.6 (2C), 136.0, 140.2, 148.6, 164.3, 164.5 ppm. MS (ESI): m/z = $391[\mathrm{M}+\mathrm{H}]^{+}$. HRMS $\left(\mathrm{TOF} \mathrm{ES}^{+}\right): \mathrm{m} / \mathrm{z}[\mathrm{M}+\mathrm{H}]^{+}$calculated for $\mathrm{C}_{23} \mathrm{H}_{22} \mathrm{~N}_{2} \mathrm{O}_{4}$ : 391.1652; found: 391.1662. Light yellow oil. $R_{\mathrm{f}}($ Ethyl acetate:Hexane, 1:2) $=0.35$. IR $(\mathrm{KBr}$ plate): $751,1208,1502,1598,1704,1729 \mathrm{~cm}^{-1}$. 


\subsection{Procedure for the detecting non-aromatic intermediate 7 and its transformation into}

pyrrole 4a and pyrrolopyrazine 5

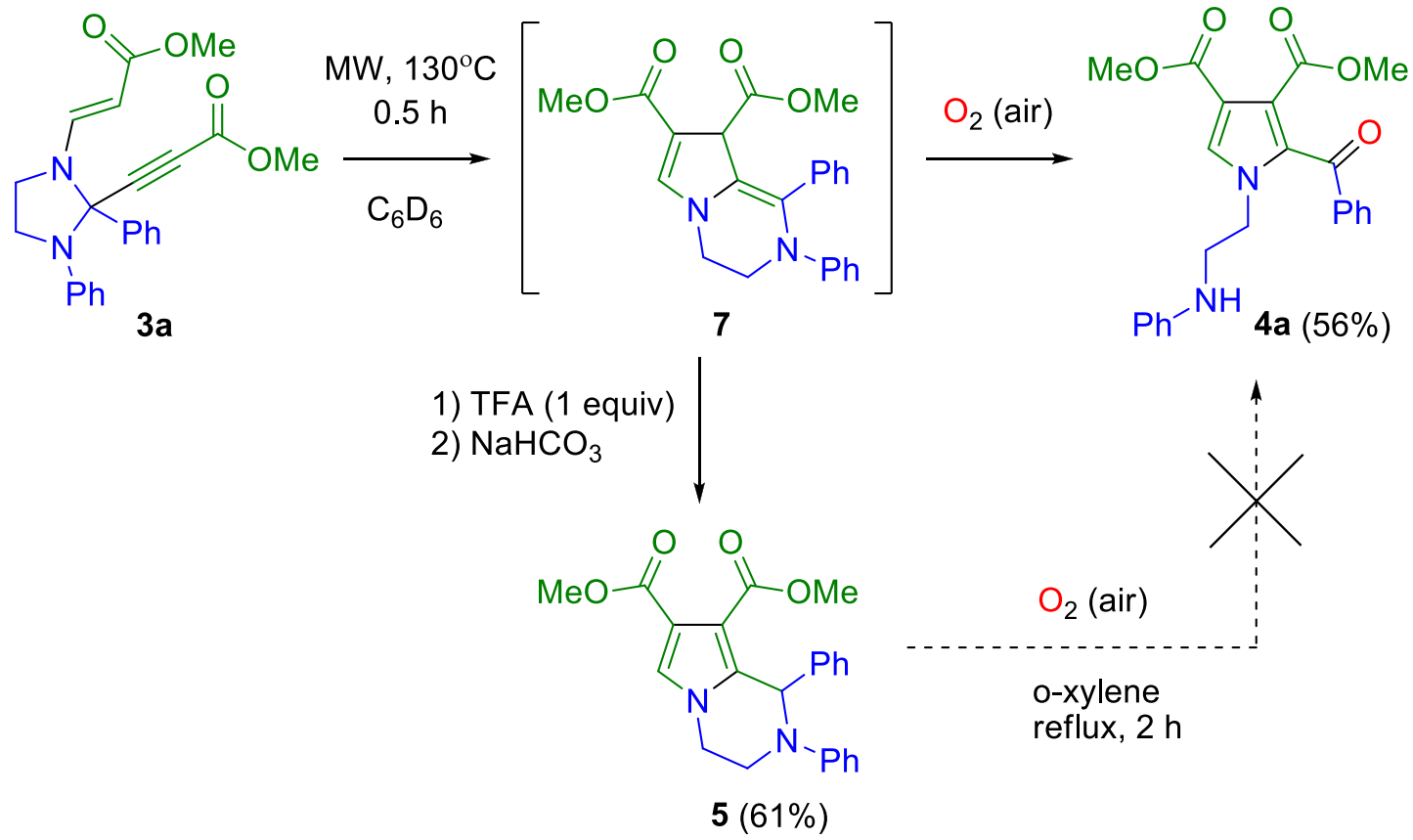

Under argon a solution of methyl (2E)-3-[2-(3-methoxy-3-oxoprop-1-yn-1-yl)-2,3diphenylimidazolidin-1-yl]prop-2-enoate 3a (195 mg, $0.5 \mathrm{mmol})$ in $\mathrm{C}_{6} \mathrm{D}_{6}(2 \mathrm{~mL})$ was irradiated in 10-mL glass vessel sealed with Teflon septum in microwave reactor Monowave 300 (Anton Paar $\mathrm{GmbH}$ ) at temperature $130^{\circ} \mathrm{C}$ for $30 \mathrm{~min}$. Then it was cooled to ambient temperature with gas jet cooling, $0.5 \mathrm{~mL}$ of the reaction mixture was immediately placed in NMR tube in argon atmosphere, and NMR spectrum was registered.

To the another part of the reaction mixture $(0.75 \mathrm{~mL}) 1$ equivalent of trifluoroacetic acid $(0.19$ mmol, $14 \mu \mathrm{L}$ ) was added, after 5 min the solution was quenched by the addition of $\mathrm{NaHCO}_{3}$ aq, then organic layer was dried over anhydrous $\mathrm{Na}_{2} \mathrm{SO}_{4}$, and $\mathrm{NMR}$ spectrum was registered. Evaporation of benzene- $\mathrm{d}_{6}$ in vacuo and purification by column chromatography with chloroform: hexane (1:1) as eluent yielded pyrrolopyrazine 5 (45mg, 61\%).

The remaining part of the reaction mixture $(0.75 \mathrm{~mL})$ was left standing in the opened vessel at room temperature for $12 \mathrm{~h}$, then benzene- $\mathrm{d}_{6}$ was evaporated in vacuo, the residue was purified by column chromatography with chloroform: hexane (1:1) as eluent yielded pyrrole $4 \mathbf{a}(42 \mathrm{mg}, 56 \%)$. 
Dimethyl 1,2-diphenyl-2,3,4,8-tetrahydropyrrolo[1,2-a]pyrazine-7,8-dicarboxylate (7)

$\mathrm{H}_{3} \mathrm{CO}_{2} \mathrm{C}$<smiles>CC1=CN2CCN(c3ccccc3)C(c3ccccc3)=C2C1CO</smiles>

${ }^{1} \mathrm{H}$ NMR $\left(\mathrm{C}_{6} \mathrm{D}_{6}, 600 \mathrm{MHz}\right): \delta 3.05$ - 3.10 (m, $\left.2 \mathrm{H}\right), 3.42$ - $3.46(\mathrm{~m}, 1 \mathrm{H})$, $3.57(\mathrm{~s}, 3 \mathrm{H}), 3.71(\mathrm{dt}, 1 \mathrm{H}, \quad J=13.1,3.0 \mathrm{~Hz}), 4.01(\mathrm{~s}, 3 \mathrm{H}), 5.68(\mathrm{~s}, 1 \mathrm{H})$, $7.08-7.12(\mathrm{~m}, 1 \mathrm{H}), 7.25-7.28(\mathrm{~m}, 3 \mathrm{H}), 7.29-7.33(\mathrm{~m}, 1 \mathrm{H}), 7.37-$ $7.40(\mathrm{~m}, 2 \mathrm{H}), 7.46-7.49(\mathrm{~m}, 2 \mathrm{H}), 7.87-7.89(\mathrm{~m}, 2 \mathrm{H}) \mathrm{ppm} .{ }^{13} \mathrm{C}\{1 \mathrm{H}\}$

NMR ( $\left.\mathrm{C}_{6} \mathrm{D}_{6}, 150 \mathrm{MHz}\right): \delta 43.6,49.0,50.5,50.5,51.6,116.0,119.9,121.9,122.8$ (2C), 126.0, 127.1, 128.4 (4C), 129.0 (2C), 130.3, 136.3, 146.5, 164.2, 171.1 ppm. MS (ESI): m/z = 389 [M$\mathrm{H}]^{+}$.

\subsection{Attempt to transform of pyrrolopyrazine 5 into pyrrole $4 a$}

A solution of dimethyl 1,2-diphenyl-1,2,3,4-tetrahydropyrrolo[1,2-a]pyrazine-7,8-dicarboxylate 5 $(195 \mathrm{mg}, 0.5 \mathrm{mmol})$ in $o$-xylene $(10 \mathrm{~mL})$ was refluxed for $2 \mathrm{~h}$ under vigorous stirring in the air using magnetic stirrer Hei-Standard (Heidolph) as the heat source. After removal of $o$-xylene in vacuo, the residue was purified by column chromatography with chloroform: hexane (1:1) as eluent yielded starting pyrrolopyrazine 5 (181mg, 93\%).

\subsection{Procedure for the thermal synthesis of pyrrolopyrazine 5 from imidazolidine $3 a$}

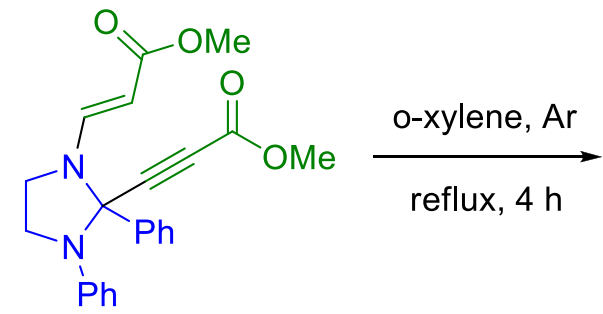

$3 a$<smiles>COC(=O)c1cn2c(c1C(=O)OC)C(c1ccccc1)N(c1ccccc1)CC2</smiles>

$5(42 \%)$

Under argon solution of methyl (2E)-3-[2-(3-methoxy-3-oxoprop-1-yn-1-yl)-2,3diphenylimidazolidin-1-yl]prop-2-enoate 3a (195mg, $0.5 \mathrm{mmol})$ in o-xylene (10 mL) was refluxed for $4 \mathrm{~h}$ under vigorous stirring using magnetic stirrer Hei-Standard (Heidolph) as the heat source. After removal of o-xylene in vacuo, the residue was purified by column chromatography with chloroform: hexane (1:1) as eluent yielded pyrrolopyrazine 5 (82mg, 42\%). 


\section{Characterization Data of Products}

\section{1 $\mathrm{N}$-aryl-2-imidazolines and 1-methyl-2-phenyl-2-imidazoline}

\section{1,2-Diphenyl-4,5-dihydro-1H-imidazole ${ }^{[4]}$ (1a)}

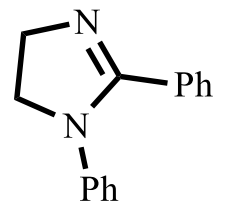

Following GP1 with $N$-benzoyl ethanolamine $\left(25 \mathrm{mmol}\right.$ ) after purification by $\mathrm{SiO}_{2}$ column chromatography $\left(\mathrm{CHCl}_{3}-\mathrm{MeOH}, 100: 0 \rightarrow 100: 1\right)$ yielded 1a $(3.85 \mathrm{~g}, 69 \%)$ as yellow solid.

${ }^{1} \mathrm{H}$ NMR $\left(\mathrm{CDCl}_{3}, 600 \mathrm{MHz}\right): \delta 4.05(\mathrm{~s}, 4 \mathrm{H}), 6.77$ - $6.81(\mathrm{~m}, 2 \mathrm{H}), 6.96$ - $7.01(\mathrm{~m}, 1 \mathrm{H}), 7.14$ - 7.17 $(\mathrm{m}, 2 \mathrm{H}), 7.26-7.29(\mathrm{~m}, 2 \mathrm{H}), 7.33-7.36(\mathrm{~m}, 1 \mathrm{H}), 7.47-7.50(\mathrm{~m}, 2 \mathrm{H}) \mathrm{ppm} .{ }^{13} \mathrm{C}\{1 \mathrm{H}\} \mathrm{NMR}$ $\left(\mathrm{CDCl}_{3}, 150 \mathrm{MHz}\right): \delta 52.9,54.1,122.7$ (2C), 123.5, 128.2 (2C), 128.8 (2C), 128.8, 130.1 (2C), 131.0, 143.1, 162.7 ppm. MS (ESI): m/z = $223[\mathrm{M}+\mathrm{H}]^{+}$. m.p. $74-75^{\circ} \mathrm{C}$. IR (KBr plate): 1074, 1124, $1273,1367,1494,1596,2871,2940,3032 \mathrm{~cm}^{-1}$.

\section{1-(4-Methoxyphenyl)-2-phenyl-4,5-dihydro-1H-imidazole ${ }^{[4]}(1 \mathrm{~b})$}

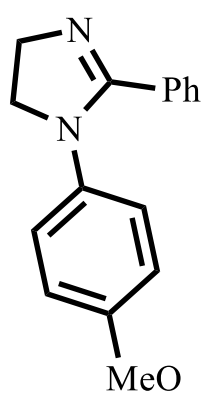

Following GP1 with $N$-benzoyl ethanolamine (25 mmol) after purification by $\mathrm{SiO}_{2}$ column chromatography $\left(\mathrm{CHCl}_{3}-\mathrm{MeOH}, 100: 0 \rightarrow 100: 1\right)$ yielded $\mathbf{1 b}(4.24 \mathrm{~g}, 67 \%)$ as brown solid.

${ }^{1} \mathrm{H}$ NMR $\left(\mathrm{CDCl}_{3}, 600 \mathrm{MHz}\right): \delta 3.73$ (s, $\left.3 \mathrm{H}\right), 3.95$ (dt, 2H, J=10.6, 9.6 Hz), 4.05 $(\mathrm{dt}, 2 \mathrm{H}, \quad J=10.6,9.6 \mathrm{~Hz}) 6.70-6.73(\mathrm{~m}, 2 \mathrm{H}),, 6.78-6.81(\mathrm{~m}, 2 \mathrm{H}), 7.24(\mathrm{t}, 2 \mathrm{H}$, $J=7.6 \mathrm{~Hz}), 7.30-7.34(\mathrm{~m}, 1 \mathrm{H}), 7.46-7.50(\mathrm{~m}, 2 \mathrm{H}) \mathrm{ppm} .{ }^{13} \mathrm{C}\{1 \mathrm{H}\} \mathrm{NMR}\left(\mathrm{CDCl}_{3}\right.$, $150 \mathrm{MHz}): \delta 53.3,55.2,55.5,114.2$ (2C), 125.3 (2C), 128.2, 128.8 (2C), 129.8 (2C), 131.3, 137.0, 156.4, 163.6 ppm. MS (ESI): $\mathrm{m} / \mathrm{z}=253[\mathrm{M}+\mathrm{H}]^{+}$. m.p. $79-80^{\circ} \mathrm{C}$. IR (KBr plate): 1245, 1272, 1381, $1513,1598,2934,3071 \mathrm{~cm}^{-1}$.

\section{1-(4-Chlorophenyl)-2-phenyl-4,5-dihydro- $1 H$-imidazole ${ }^{[4]}(1 \mathrm{c})$}

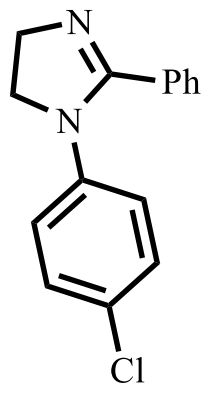

Following GP1 with $N$-benzoyl ethanolamine (25 mmol) after purification by $\mathrm{SiO}_{2}$ column chromatography $\left(\mathrm{CHCl}_{3}-\mathrm{MeOH}, 100: 0 \rightarrow 100: 1\right)$ yielded $1 \mathrm{c}(3.56 \mathrm{~g}, 50 \%)$ as yellow solid.

${ }^{1} \mathrm{H}$ NMR $\left(\mathrm{CDCl}_{3}, 600 \mathrm{MHz}\right): \delta 3.97$ - 4.07 (m, $\left.4 \mathrm{H}\right)$, 6.67-6.70 (m, $\left.2 \mathrm{H}\right)$ 7.08-7.11 (m, $2 \mathrm{H}), 7.26-7.30(\mathrm{~m}, 2 \mathrm{H}), 7.34-7.38(\mathrm{~m}, 1 \mathrm{H}), 7.43-7.47(\mathrm{~m}, 2 \mathrm{H}) \mathrm{ppm} .{ }^{13} \mathrm{C}$ $\{1 \mathrm{H}\} \mathrm{NMR}\left(\mathrm{CDCl}_{3}, 150 \mathrm{MHz}\right): \delta 53.2,53.9,123.6(2 \mathrm{C}), 128.3,128.4(2 \mathrm{C}), 128.7$ (2C), 128.8, 130.2 (2C), 131.0, 141.8, 162.2 ppm. MS (ESI): m/z = $285[\mathrm{M}+\mathrm{H}]^{+}$. m.p. 95-96 ${ }^{\circ} \mathrm{C}$. IR (KBr plate): 704, 772, 824, 1093, 1272, 1321, 1380, 1493, 1594, 1621, 2874, 2946, $3061 \mathrm{~cm}^{-1}$. 


\section{2-Phenyl-1-[4-(trifluoromethyl)phenyl]-4,5-dihydro-1H-imidazole (1d)}

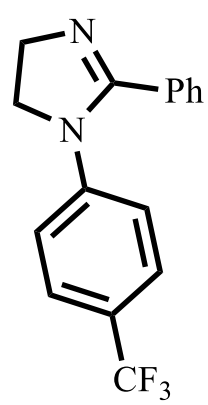

Following GP1 with $N$-benzoyl ethanolamine (25 mmol) after purification by $\mathrm{SiO}_{2}$ column chromatography $\left(\mathrm{CHCl}_{3}-\mathrm{MeOH}, 100: 0 \rightarrow 100: 1\right)$ yielded $\mathbf{1 d}(6.55 \mathrm{~g}, 90 \%)$ as brown oil.

${ }^{1} \mathrm{H}$ NMR $\left(\mathrm{CDCl}_{3}, 600 \mathrm{MHz}\right): \delta 4.05-4.12(\mathrm{~m}, 4 \mathrm{H}), 6.76(\mathrm{~d}, 2 \mathrm{H}, J=8.6 \mathrm{~Hz}), 7.30$ $7.35(\mathrm{~m}, 2 \mathrm{H}), 7.37$ (d, 2H, J=8.6 Hz), $7.40(\mathrm{tt}, 1 \mathrm{H}, J=7.1,1.0 \mathrm{~Hz}), 7.46-7.49$ (m,

$2 \mathrm{H})$ ppm. ${ }^{13} \mathrm{C}\{1 \mathrm{H}\}$ NMR $\left(\mathrm{CDCl}_{3}, 150 \mathrm{MHz}\right): \delta 53.2(2 \mathrm{C}), 120.8(2 \mathrm{C}), 124.2(\mathrm{q}$, $\left.J_{C F}=31.8 \mathrm{~Hz}\right), 125.9,125.9\left(\mathrm{q}, J_{C F}=4.3 \mathrm{~Hz}\right), 128.6(2 \mathrm{C}), 128.6(2 \mathrm{C}), 130.4(2 \mathrm{C}), 131.1,145.6$, $161.5 \mathrm{ppm}$. MS (ESI): $\mathrm{m} / \mathrm{z}=291[\mathrm{M}+\mathrm{H}]^{+}$. HRMS $\left(\mathrm{TOF} \mathrm{ES}^{+}\right): \mathrm{m} / \mathrm{z}[\mathrm{M}+\mathrm{H}]^{+}$calculated for $\mathrm{C}_{16} \mathrm{H}_{13} \mathrm{~F}_{3} \mathrm{~N}_{2}$ : 291.1103; found: 291.1104. $\mathrm{R}_{\mathrm{f}}\left(\mathrm{CHCl}_{3}: \mathrm{MeOH}: \mathrm{NH}_{3}, 10: 1: 0.1\right)=0.4$. IR ( $\mathrm{KBr}$ plate): $698,772,836,1071,1118,1323,1378,1522,1612,2639,2873,2942 \mathrm{~cm}^{-1}$.

\section{1-(2-Methylphenyl)-2-phenyl-4,5-dihydro- $1 H$-imidazole ${ }^{[4]}(1 \mathrm{e})$}

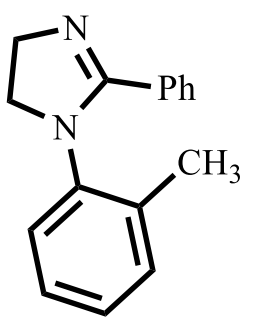

Following GP1 with $N$-benzoyl ethanolamine $(25 \mathrm{mmol})$ after purification by

$\mathrm{SiO}_{2}$ column chromatography $\left(\mathrm{CHCl}_{3}-\mathrm{MeOH}, 100: 0 \rightarrow 100: 1\right)$ yielded $1 \mathrm{e}(5.09 \mathrm{~g}$, $86 \%$ ) as yellow solid.

${ }^{1} \mathrm{H}$ NMR $\left(\mathrm{CDCl}_{3}, 600 \mathrm{MHz}\right): \delta 2.35$ (s, $\left.3 \mathrm{H}\right), 3.54$ (br., $\left.1 \mathrm{H}\right), 4.10$ (br., $\left.3 \mathrm{H}\right), 6.78$ $(\mathrm{dd}, 1 \mathrm{H}, J=7.8,1.3 \mathrm{~Hz}), 6.98(\mathrm{td}, 1 \mathrm{H}, J=7.6,1.0 \mathrm{~Hz}), 7.06(\mathrm{td}, 1 \mathrm{H}, J=7.4,1.3$ $\mathrm{Hz}), 7.16$ - $7.21(\mathrm{~m}, 3 \mathrm{H}), 7.23-7.28(\mathrm{~m}, 1 \mathrm{H}), 7.43(\mathrm{dd}, 2 \mathrm{H}, J=8.3,1.3 \mathrm{~Hz}) \mathrm{ppm} .{ }^{13} \mathrm{C}\{1 \mathrm{H}\} \mathrm{NMR}$ $\left(\mathrm{CDCl}_{3}, 150 \mathrm{MHz}\right): \delta 18.3,54.1,54.5,126.4,126.9,127.3$ (2C), 128.0, 128.5 (2C), 129.8, 131.1, 131.1, 135.1, 143.3, 165.1 ppm. MS (ESI): $\mathrm{m} / \mathrm{z}=237[\mathrm{M}+\mathrm{H}]^{+}$. m.p. 86-87 ${ }^{\circ} \mathrm{C}$. IR (KBr plate): 691, $763,996,1110,1276,1374,1488,1611,2876,2947 \mathrm{~cm}^{-1}$.

\section{1-Methyl-2-phenyl-4,5-dihydro-1H-imidazole ${ }^{[5]}(1 \mathrm{f})$}

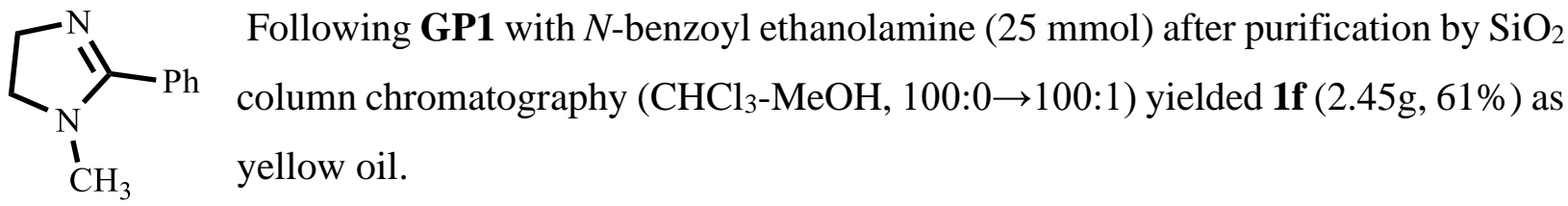

${ }^{1} \mathrm{H} \mathrm{NMR}\left(\mathrm{CDCl}_{3}, 600 \mathrm{MHz}\right): \delta 2.68$ (s, $\left.3 \mathrm{H}\right), 3.30$ - 3.34 (m, $\left.2 \mathrm{H}\right), 3.74$ - 3.77 (m, $\left.2 \mathrm{H}\right), 7.27$ - 7.30 $(\mathrm{m}, 3 \mathrm{H}), 7.44-7.47(\mathrm{~m}, 2 \mathrm{H}) \mathrm{ppm} .{ }^{13} \mathrm{C}\{1 \mathrm{H}\} \mathrm{NMR}\left(\mathrm{CDCl}_{3}, 150 \mathrm{MHz}\right): \delta 36.1,53.1,53.9,128.0$ (2C), 128.1 (2C), 129.5, 131.2, 168.0; MS (ESI): m/z = $161[\mathrm{M}+\mathrm{H}]^{+} . \mathrm{R}_{\mathrm{f}}\left(\mathrm{CHCl}_{3}: \mathrm{MeOH}: \mathrm{NH}_{3}\right.$, 10:1:0.1)=0.4. IR (KBr plate): 703, 777, 1025, 1062, 1278, 1332, 1389, 1448, 1571, 1594, 1612, $2862,2932,3389 \mathrm{~cm}^{-1}$. 


\section{2 $N$-Benzyl-2-Imidazolines}

\section{1-Benzyl-2-phenyl-4,5-dihydro-1H-imidazole (1g)}

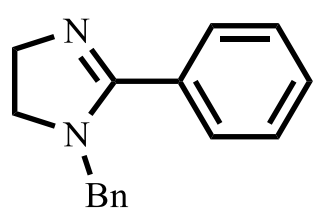

Following GP2 with $N$-benzylethylenediamine $(40 \mathrm{mmol})$ after purification by $\mathrm{SiO}_{2}$ column chromatography $\left(\mathrm{CHCl}_{3}-\mathrm{MeOH}, 100: 0 \rightarrow 100: 1\right)$ yielded $\mathbf{1 g}$ $(7.84 \mathrm{~g}, 87 \%)$ as yellow solid.

${ }^{1} \mathrm{H} \mathrm{NMR}\left(\mathrm{CDCl}_{3}, 600 \mathrm{MHz}\right): \delta 3.41(\mathrm{t}, 2 \mathrm{H}, J=9.8 \mathrm{~Hz}), 3.93(\mathrm{t}, 2 \mathrm{H}, J=9.8 \mathrm{~Hz}), 4.31(\mathrm{~s}, 2 \mathrm{H}), 7.26$ - $7.30(\mathrm{~m}, 3 \mathrm{H}), 7.34$ - 7.37 (m, $2 \mathrm{H}), 7.39$ - $7.43(\mathrm{~m}, 3 \mathrm{H}), 7.57$ - $7.64(\mathrm{~m}, 2 \mathrm{H}) \mathrm{ppm} .{ }^{13} \mathrm{C}\{1 \mathrm{H}\}$ NMR ( $\left.\mathrm{CDCl}_{3}, 150 \mathrm{MHz}\right): \delta 51.1,53.1,53.2,127.2$ (2C), 127.5, 128.2 (2C), 128.6 (2C), 128.8 (2C), 130.2, 131.0, 138.0, 167.5 ppm. MS (ESI): m/z = $237[\mathrm{M}+\mathrm{H}]^{+} . \quad \mathrm{HRMS}\left(\mathrm{TOF} \mathrm{ES}^{+}\right): \mathrm{m} / \mathrm{z}$ $[\mathrm{M}+\mathrm{H}]^{+}$calculated for $\mathrm{C}_{16} \mathrm{H}_{16} \mathrm{~N}_{2}$ : 237.1386; found: 237.1386. m.p. 64-65 ${ }^{\circ} \mathrm{C}$. IR (KBr plate): 1130, $1249,1361,1403,1454,1494,1610,1709,2869,2929,3054 \mathrm{~cm}^{-1}$.

\section{1-Benzyl-2-(4-methoxyphenyl)-4,5-dihydro-1H-imidazole (1h)}

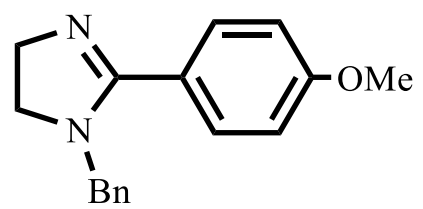

Following GP2 with $N$-benzylethylenediamine $(40 \mathrm{mmol})$ after purification by $\mathrm{SiO}_{2}$ column chromatography $\left(\mathrm{CHCl}_{3}-\mathrm{MeOH}\right.$, $100: 0 \rightarrow 100: 1)$ yielded $\mathbf{1 h}(8.83 \mathrm{~g}, 87 \%)$ as yellow solid.

${ }^{1} \mathrm{H}$ NMR $\left(\mathrm{CDCl}_{3}, 600 \mathrm{MHz}\right): \delta 3.39(\mathrm{t}, 2 \mathrm{H}, J=9.8 \mathrm{~Hz}), 3.81(\mathrm{~s}, 3 \mathrm{H}), 3.90(\mathrm{t}, 2 \mathrm{H}, J=9.8 \mathrm{~Hz}), 4.31$ (s, $2 \mathrm{H}), 6.88-6.92(\mathrm{~m}, 2 \mathrm{H}), 7.26-7.30(\mathrm{~m}, 3 \mathrm{H}), 7.34-7.37$ (m, $2 \mathrm{H}), 7.54$ - $7.56(\mathrm{~m}, 2 \mathrm{H}) \mathrm{ppm} .{ }^{13} \mathrm{C}$ $\{1 \mathrm{H}\} \mathrm{NMR}\left(\mathrm{CDCl}_{3}, 150 \mathrm{MHz}\right): \delta 51.4,53.2,53.5,55.4,114.0(2 \mathrm{C}), 123.5,127.2(2 \mathrm{C}), 127.4$, 128.8 (2C), 129.8 (2C), 138.3, 161.0, 167.3 ppm. MS (ESI): m/z = $267[\mathrm{M}+\mathrm{H}]^{+}$. HRMS (TOF $\mathrm{ES}^{+}$): $\mathrm{m} / \mathrm{z}[\mathrm{M}+\mathrm{H}]^{+}$calculated for $\mathrm{C}_{17} \mathrm{H}_{19} \mathrm{~N}_{2} \mathrm{O}$ : 267.1492; found: 267.1491. m.p. 93-94 ${ }^{\circ} \mathrm{C}$. IR $(\mathrm{KBr}$ plate): $1185,1249,1393,1514,1605,2842,2940,3007,3058 \mathrm{~cm}^{-1}$.

\section{1-Benzyl-2-(4-chlorophenyl)-4,5-dihydro-1H-imidazole (1i)}

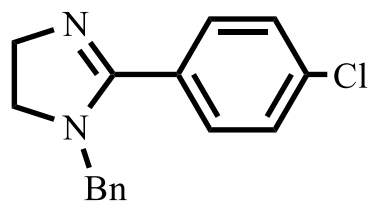

Following GP2 with $N$-benzylethylenediamine $(40 \mathrm{mmol})$ after purification by $\mathrm{SiO}_{2}$ column chromatography $\left(\mathrm{CHCl}_{3}-\mathrm{MeOH}\right.$, $100: 0 \rightarrow 100: 1)$ yielded $1 \mathbf{i}(7.11 \mathrm{~g}, 69 \%)$ as yellow solid.

${ }^{1} \mathrm{H} \mathrm{NMR}\left(\mathrm{CDCl}_{3}, 600 \mathrm{MHz}\right): \delta 3.41(\mathrm{t}, 2 \mathrm{H}, J=9.8 \mathrm{~Hz}), 3.92(\mathrm{t}, 2 \mathrm{H}, J=10.1 \mathrm{~Hz}), 4.27(\mathrm{~s}, 2 \mathrm{H}), 7.25$ $(\mathrm{d}, 2 \mathrm{H}, J=7.6 \mathrm{~Hz}), 7.29(\mathrm{t}, 1 \mathrm{H}, J=7.1 \mathrm{~Hz}), 7.34-7.39(\mathrm{~m}, 4 \mathrm{H}), 7.55$ (d, 2H, J=8.6 Hz) ppm. ${ }^{13} \mathrm{C}$ $\{1 \mathrm{H}\} \mathrm{NMR}\left(\mathrm{CDCl}_{3}, 150 \mathrm{MHz}\right): \delta 51.3,53.2,53.6,127.1$ (2C), 127.5 (2C), 128.9 (2C), 128.9, 129.6 (2C), 129.8, 136.0, 137.9, 166.5 ppm. MS (ESI): m/z = $271[\mathrm{M}+\mathrm{H}]^{+}$. HRMS (TOF ES ${ }^{+}$): $\mathrm{m} / \mathrm{z}[\mathrm{M}+\mathrm{H}]^{+}$calculated for $\mathrm{C}_{16} \mathrm{H}_{15} \mathrm{ClN}_{2}$ : 271.0996; found: 271.0991 . m.p. $112-113^{\circ} \mathrm{C}$. IR (KBr plate): $727,834,1011,1087,1249,1405,1494,1593,1612,2866,2927 \mathrm{~cm}^{-1}$. 
1-Benzyl-2-(4-nitrophenyl)-4,5-dihydro-1H-imidazole (1j)

$\underset{\mathrm{Bn}}{\mathrm{N}}=\mathrm{NO}_{2}$

Following GP2 with $N$-benzylethylenediamine $(40 \mathrm{mmol})$ after

purification by $\mathrm{SiO}_{2}$ column chromatography $\left(\mathrm{CHCl}_{3}-\mathrm{MeOH}\right.$, $100: 0 \rightarrow 100: 1)$ yielded $\mathbf{1 j}(9.54 \mathrm{~g}, 89 \%)$ as yellow solid.

${ }^{1} \mathrm{H} \mathrm{NMR}\left(\mathrm{CDCl}_{3}, 600 \mathrm{MHz}\right): \delta 3.46$ (t, 2H, J=10.1 Hz), 3.97 (t, 2H, J=10.1 Hz), 4.26 (s, $\left.2 \mathrm{H}\right)$, $7.25(\mathrm{~d}, 2 \mathrm{H}, J=7.6 \mathrm{~Hz}), 7.31(\mathrm{t}, 1 \mathrm{H}, J=7.1 \mathrm{~Hz}), 7.36(\mathrm{t}, 2 \mathrm{H}, J=7.6 \mathrm{~Hz}), 7.78(\mathrm{~d}, 2 \mathrm{H}, J=8.6 \mathrm{~Hz}), 8.25$ $(\mathrm{d}, 2 \mathrm{H}, J=8.6 \mathrm{~Hz}) \mathrm{ppm} .{ }^{13} \mathrm{C}\{1 \mathrm{H}\} \mathrm{NMR}\left(\mathrm{CDCl}_{3}, 150 \mathrm{MHz}\right): \delta 51.4,53.1,54.0,123.8(2 \mathrm{C}), 127.1$ (2C), 127.7 (2C), 129.0, 129.4 (2C), 137.4, 137.8, 148.8, 165.6; MS (ESI): m/z = $282[\mathrm{M}+\mathrm{H}]^{+}$; HRMS (TOF ES ${ }^{+}$): m/z [M+H] $]^{+}$calculated for $\mathrm{C}_{16} \mathrm{H}_{15} \mathrm{~N}_{3} \mathrm{O}_{2}$ : 282.1237; found: 282.1235. m.p. 101$102^{\circ} \mathrm{C}$. IR (KBr plate): 700, 746, 868, 1336, 1495, 1519, 1580, 2867, 2941, $3067 \mathrm{~cm}^{-1}$.

\section{1-Benzyl-2-(thiophen-2-yl)-4,5-dihydro-1H-imidazole ${ }^{[6]}(1 \mathrm{k})$}

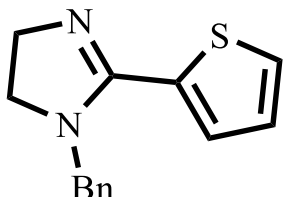

Following GP2 with $N$-benzylethylenediamine ( $40 \mathrm{mmol}$ ) after purification by $\mathrm{SiO}_{2}$ column chromatography $\left(\mathrm{CHCl}_{3}-\mathrm{MeOH}, \quad 100: 0 \rightarrow 100: 1\right)$ yielded $\mathbf{1 k}$ $(6.74 \mathrm{~g}, 73 \%)$ as yellow solid.

${ }^{1} \mathrm{H} \mathrm{NMR}\left(\mathrm{CDCl}_{3}, 600 \mathrm{MHz}\right): \delta 3.50(\mathrm{t}, 2 \mathrm{H}, J=10.1 \mathrm{~Hz}), 3.93(\mathrm{t}, 2 \mathrm{H}, J=10.3 \mathrm{~Hz}), 4.57(\mathrm{~s}, 2 \mathrm{H})$, 7.03 - $7.06(\mathrm{~m}, 1 \mathrm{H}), 7.28$ - $7.32(\mathrm{~m}, 3 \mathrm{H}), 7.37$ (t, 2H, J=7.6 Hz), $7.41-7.45(\mathrm{~m}, 2 \mathrm{H}) \mathrm{ppm} .{ }^{13} \mathrm{C}$ $\{1 \mathrm{H}\} \mathrm{NMR}\left(\mathrm{CDCl}_{3}, 150 \mathrm{MHz}\right): \delta 51.8,52.2,53.0,127.1(2 \mathrm{C}), 127.5,127.7(2 \mathrm{C}), 128.8,128.9$, 129.0, 131.7, 137.5, 160.9 ppm. MS (ESI): m/z = $243[\mathrm{M}+\mathrm{H}]^{+}$. HRMS (TOF ES $\left.{ }^{+}\right): \mathrm{m} / \mathrm{z}[\mathrm{M}+\mathrm{H}]^{+}$ calculated for $\mathrm{C}_{14} \mathrm{H}_{14} \mathrm{~N}_{2} \mathrm{~S}$ : 243.0950; found: 243.0950. m.p. 52-53 ${ }^{\circ} \mathrm{C}$. IR (KBr plate): 700, 715, 732, 850, 999, 1266, 1358, 1443, 1522, 1597, 1709, 2865, 2930, $3054 \mathrm{~cm}^{-1}$.

\section{1-Benzyl-2-methyl-4,5-dihydro-1H-imidazole ${ }^{[3]}$ (11)}

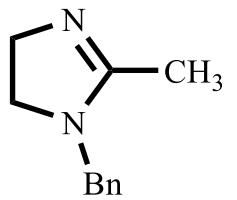

A solution of $N$-benzylethylenediamine $(40 \mathrm{mmol})$ and ethyl acetimidate hydrochloride $(40 \mathrm{mmol})$ in dry acetonitrile $(40 \mathrm{ml})$ was refluxed for $3 \mathrm{~h}$. After removal of acetonitrile in vacuo the crude hydrochloride was taken up in the minimum of water, treated with excess of sodium hydroxide solution $(30 \% \mathrm{w} / \mathrm{v})$ and the mixture extracted with chloroform $(2 \times 100 \mathrm{ml})$. The organic layer was dried over anhydrous $\mathrm{Na}_{2} \mathrm{SO}_{4}$, solvent evaporated in vacuo, and the residue fractionally distilled under reduced pressure yielded $11(5.08 \mathrm{~g}, 73 \%)$ as colourless oil.

${ }^{1} \mathrm{H} \mathrm{NMR}\left(\mathrm{CDCl}_{3}, 600 \mathrm{MHz}\right): \delta 2.10(\mathrm{~s}, 3 \mathrm{H}), 3.29(\mathrm{t}, 2 \mathrm{H}, J=9.8 \mathrm{~Hz}), 3.71(\mathrm{t}, 2 \mathrm{H}, J=9.8 \mathrm{~Hz}), 4.33$ $(\mathrm{s}, 2 \mathrm{H}), 7.22(\mathrm{~d}, 2 \mathrm{H}, J=7.1 \mathrm{~Hz}), 7.27-7.31(\mathrm{~m}, 1 \mathrm{H}), 7.33-7.38(\mathrm{~m}, 2 \mathrm{H}) \mathrm{ppm} .{ }^{13} \mathrm{C}\{1 \mathrm{H}\} \mathrm{NMR}$ $\left(\mathrm{CDCl}_{3}, 150 \mathrm{MHz}\right): \delta 14.5,50.0,50.9,52.2,127.3,127.5$ (2C), 128.8 (2C), 137.8, $164.2 \mathrm{ppm}$. 


\subsection{Imidazolidines 3}

Methyl (2E)-3-[2-(3-methoxy-3-oxoprop-1-yn-1-yl)-2,3-diphenylimidazolidin-1-yl]prop-2enoate (3a)

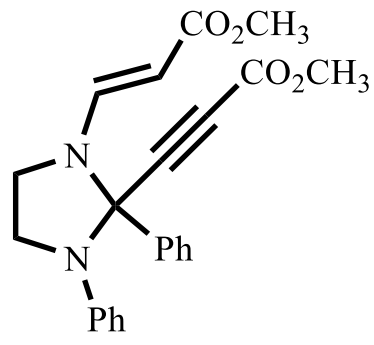

Following GP3 with 1,2-diphenyl-4,5-dihydro- $1 H$-imidazole 1a (1.7 mmol) after purification by $\mathrm{SiO}_{2}$ column chromatography $\left(\mathrm{CHCl}_{3}:\right.$ Hexane, $\left.1: 1\right)$ yielded $\mathbf{3 a}(0.58 \mathrm{~g}, 88 \%)$ as white solid.

${ }^{1} \mathrm{H} \mathrm{NMR}\left(\mathrm{CDCl}_{3}, 600 \mathrm{MHz}\right): \delta 3.58$ - $3.62(\mathrm{~m}, 1 \mathrm{H}), 3.61$ (s, 3H), 3.69 -

3.75 (m, 2 H), 3.75 (s, 3 H), 4.08 - $4.12(\mathrm{~m}, 1 \mathrm{H}), 4.77$ (d, 1H, J=13.6 Hz), $6.61(\mathrm{dd}, 2 \mathrm{H}, J=9.1,1.0 \mathrm{~Hz}), 6.81-6.84(\mathrm{~m}, 1 \mathrm{H}), 7.07-7.13(\mathrm{~m}, 2 \mathrm{H}), 7.18(\mathrm{~d}, 1 \mathrm{H}, J=13.1 \mathrm{~Hz})$, 7.40 - $7.44(\mathrm{~m}, 3 \mathrm{H}), 7.69$ - $7.72(\mathrm{~m}, 2 \mathrm{H}) \mathrm{ppm} .{ }^{13} \mathrm{C}\{1 \mathrm{H}\} \mathrm{NMR}\left(\mathrm{CDCl}_{3}, 150 \mathrm{MHz}\right): \delta$ 44.5, 47.0, 50.9, 53.0, 79.2, 79.6, 82.5, 91.4, 116.9 (2C), 120.6, 127.6 (2C), 128.9 (2C), 129.4 (2C), 130.2, 138.1, 143.4, 144.1, 153.4, 168.7 ppm. MS (ESI): m/z = $391[\mathrm{M}+\mathrm{H}]^{+}$. HRMS $\left(\mathrm{TOF} \mathrm{ES}^{+}\right): \mathrm{m} / \mathrm{z}$ $[\mathrm{M}+\mathrm{H}]^{+}$calculated for $\mathrm{C}_{23} \mathrm{H}_{22} \mathrm{~N}_{2} \mathrm{O}_{4}: 391.1652$; found: 391.1652 . m.p. $130-131^{\circ} \mathrm{C}$. IR (KBr plate): 1162, 1251, 1311, 1440, 1507, 1608, 1716, 2233, 2843, 2877, 2946, 3033, $3421 \mathrm{~cm}^{-1}$.

\section{Methyl (2E)-3-[2-(3-methoxy-3-oxoprop-1-yn-1-yl)-3-(4-methoxyphenyl)-2-}

phenylimidazolidin-1-yl]prop-2-enoate (3b)

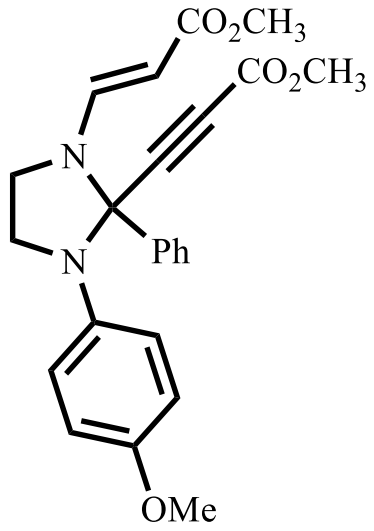

Following GP3 with 1-(4-methoxyphenyl)-2-phenyl-4,5-dihydro- $1 H$ imidazole 1 b $(1.7 \mathrm{mmol})$ after purification by $\mathrm{SiO}_{2}$ column chromatography $\left(\mathrm{CHCl}_{3}:\right.$ Hexane, $\left.1: 1\right)$ yielded $\mathbf{3 b}(0.66 \mathrm{~g}, 92 \%)$ as brown solid.

${ }^{1} \mathrm{H}$ NMR $\left(\mathrm{CDCl}_{3}, 600 \mathrm{MHz}\right): \delta 3.55$ - 3.58 (m, $\left.1 \mathrm{H}\right), 3.59$ (s, $\left.3 \mathrm{H}\right), 3.67-$ 3.73 (m, 2 H), 3.68 (s, $3 \mathrm{H}), 3.75$ (s, $3 \mathrm{H}), 3.86-3.90$ (m, 1H), 4.73 (d, 1H, $J=13.6 \mathrm{~Hz}), 6.57(\mathrm{~d}, 2 \mathrm{H}, J=9.1 \mathrm{~Hz}), 6.66(\mathrm{~d}, 2 \mathrm{H}, J=9.1 \mathrm{~Hz}), 7.16(\mathrm{~d}, 1 \mathrm{H}$, $J=13.1 \mathrm{~Hz}), 7.41(\mathrm{t}, 3 \mathrm{H}, \quad J=3.0 \mathrm{~Hz}), 7.63-7.68(\mathrm{~m}, 2 \mathrm{H}) \mathrm{ppm} .{ }^{13} \mathrm{C}\{1 \mathrm{H}\}$

NMR ( $\left.\mathrm{CDCl}_{3}, 150 \mathrm{MHz}\right): \delta 44.9,47.6,50.8,53.0,55.4,79.8,80.8,82.5,90.4,114.1$ (2C), 120.4 (2C), 128.1 (2C), 129.2 (2C), 130.2, 136.8, 138.2, 144.4, 153.4, 155.0, 168.8 ppm. MS (ESI): m/z $=421[\mathrm{M}+\mathrm{H}]^{+}$. HRMS $\left(\mathrm{TOF} \mathrm{ES}^{+}\right): \mathrm{m} / \mathrm{z}[\mathrm{M}+\mathrm{H}]^{+}$calculated for $\mathrm{C}_{24} \mathrm{H}_{24} \mathrm{~N}_{2} \mathrm{O}_{5}: 421.1757$; found: 421.1758. m.p. $110-111^{\circ} \mathrm{C}$. IR (KBr plate): 1169, 1249, 1509, 1609, 1687, 1720, 2230, 2837, $2953,3003,3420 \mathrm{~cm}^{-1}$. 
Methyl (2E)-3-[3-(4-chlorophenyl)-2-(3-methoxy-3-oxoprop-1-yn-1-yl)-2-

\section{phenylimidazolidin-1-yl]prop-2-enoate (3c)}

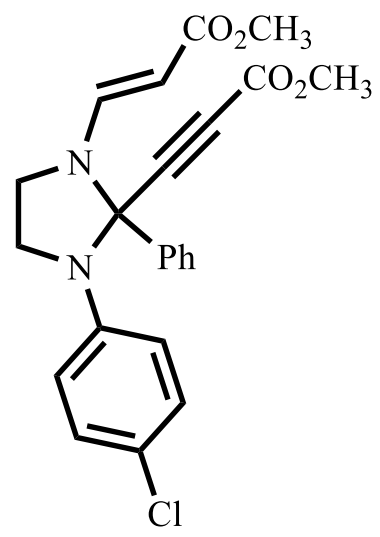

Following GP3 with 1-(4-chlorophenyl)-2-phenyl-4,5-dihydro- $1 \mathrm{H}$ imidazole 1c $(1.7 \mathrm{mmol})$ after purification by $\mathrm{SiO}_{2}$ column chromatography $\left(\mathrm{CHCl}_{3}:\right.$ Hexane, $\left.1: 1\right)$ yielded $3 \mathbf{c}(0.65 \mathrm{~g}, 90 \%)$ as yellow solid.

${ }^{1} \mathrm{H}$ NMR $\left(\mathrm{CDCl}_{3}, 600 \mathrm{MHz}\right): \delta 3.57$ - 3.62 (m, $\left.1 \mathrm{H}\right), 3.60$ (s, $\left.3 \mathrm{H}\right), 3.68$ 3.74 (m, 2 H), 3.76 (s, 3 H), 4.02 - 4.08 (m, 1 H), 4.77 (d, 1H, J=13.1 Hz), $6.52(\mathrm{~d}, 2 \mathrm{H}, J=9.1 \mathrm{~Hz}), 7.05(\mathrm{~d}, 2 \mathrm{H}, J=9.1 \mathrm{~Hz}), 7.15(\mathrm{~d}, 1 \mathrm{H}, J=13.1 \mathrm{~Hz})$, $7.41-7.44(\mathrm{~m}, 3 \mathrm{H}), 7.64-7.68(\mathrm{~m}, 2 \mathrm{H}) \mathrm{ppm} .{ }^{13} \mathrm{C}\{1 \mathrm{H}\} \mathrm{NMR}\left(\mathrm{CDCl}_{3}\right.$, $150 \mathrm{MHz}): \delta 44.4,47.1,50.9,53.1,79.3,79.6,82.0,91.7,118.0$ (2C), 125.8, 127.6 (2C), 128.8 (2C), 129.5 (2C), 130.4, 137.7, 141.9, 143.9, 153.2, 168.6 ppm. HRMS (TOF ES $\left.{ }^{+}\right):$m/z [M+H] ${ }^{+}$ calculated for $\mathrm{C}_{23} \mathrm{H}_{21} \mathrm{ClN}_{2} \mathrm{O}_{4}$ : 425.1263; found: 425.1265. m.p. $135-136^{\circ} \mathrm{C}$. IR (KBr plate): 749 , $808,1371,1500,1614,1714,2228,2869,2948,2995,3399 \mathrm{~cm}^{-1}$.

\section{Methyl (2E)-3-\{2-(3-methoxy-3-oxoprop-1-yn-1-yl)-2-phenyl-3-[4-}

(trifluoromethyl)phenyl]imidazolidin-1-yl\}prop-2-enoate (3d)

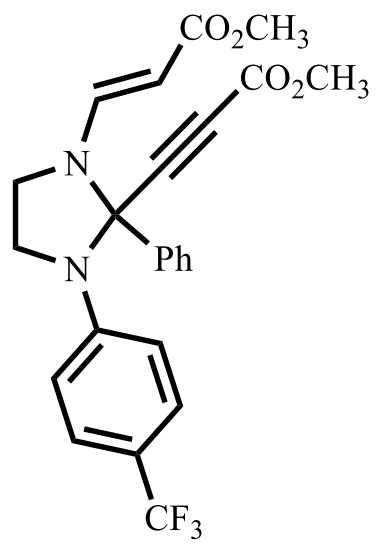

Following GP3 with 2-phenyl-1-[4-(trifluoromethyl)phenyl]-4,5dihydro- $1 H$-imidazole $\mathbf{1 d}(1.7 \mathrm{mmol})$ after purification by $\mathrm{SiO}_{2}$ column chromatography $\left(\mathrm{CHCl}_{3}:\right.$ Hexane, $\left.1: 1\right)$ yielded $\mathbf{3 d}(0.82 \mathrm{~g}, 95 \%)$ as yellow oil. The reaction time was $24 \mathrm{~h}$.

${ }^{1} \mathrm{H}$ NMR $\left(\mathrm{CDCl}_{3}, 600 \mathrm{MHz}\right): \delta 3.60$ - 3.64 (m, $\left.1 \mathrm{H}\right), 3.61$ (s, $\left.3 \mathrm{H}\right), 3.74$ 3.81 (m, 2 H), 3.77 (s, $3 \mathrm{H}), 4.14$ - 4.19 (m, $1 \mathrm{H}), 4.82$ (d, 1H, J=13.1 Hz), $6.64(\mathrm{~d}, 2 \mathrm{H}, J=9.1 \mathrm{~Hz}), 7.16(\mathrm{~d}, 1 \mathrm{H}, J=13.1 \mathrm{~Hz}), 7.35$ (d, 2H, $J=8.6 \mathrm{~Hz})$,

$7.42-7.45(\mathrm{~m}, 3 \mathrm{H}), 7.66-7.68(\mathrm{~m}, 2 \mathrm{H}) \mathrm{ppm} .{ }^{13} \mathrm{C}\{1 \mathrm{H}\} \mathrm{NMR}\left(\mathrm{CDCl}_{3}\right.$, $150 \mathrm{MHz}): \delta 44.2,46.9,51.0,53.2,79.0,79.0,81.8,92.7,115.2(2 \mathrm{C}), 121.4$ (q, $\left.J_{C F}=33.2 \mathrm{~Hz}\right)$, 125.5, 126.2 (q, $\left.J_{C F}=4.3 \mathrm{~Hz}\right), 127.4(2 \mathrm{C}), 129.6(2 \mathrm{C}), 130.5(2 \mathrm{C}), 137.3,143.7,145.7,153.2,168.5$ ppm. MS (ESI): $\mathrm{m} / \mathrm{z}=459[\mathrm{M}+\mathrm{H}]^{+}$. HRMS $\left(\mathrm{TOF} \mathrm{ES}^{+}\right): \mathrm{m} / \mathrm{z}[\mathrm{M}+\mathrm{H}]^{+}$calculated for $\mathrm{C}_{24} \mathrm{H}_{21} \mathrm{~F}_{3} \mathrm{~N}_{2} \mathrm{O}_{4}$ : 459.1526; found: 459.1526. $\mathrm{R}_{\mathrm{f}}\left(\mathrm{CHCl}_{3}\right)=0.34$. IR ( $\mathrm{KBr}$ plate): 759, 817, 1070, 1110, 1206, 1329, $1529,1615,1712,1729,2951 \mathrm{~cm}^{-1}$. 
Methyl (2E)-3-[2-(3-methoxy-3-oxoprop-1-yn-1-yl)-3-(2-methylphenyl)-2-

phenylimidazolidin-1-yl]prop-2-enoate (3e)

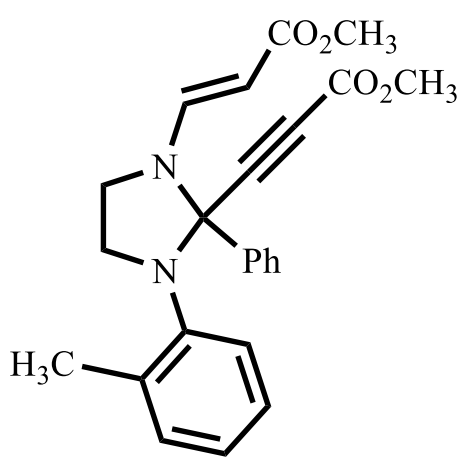

Following GP3 with 1-(2-methylphenyl)-2-phenyl-4,5-dihydro- $1 \mathrm{H}$ imidazole 1e $(1.7 \mathrm{mmol})$ after purification by $\mathrm{SiO}_{2}$ column chromatography $\left(\mathrm{CHCl}_{3}:\right.$ Hexane, $\left.1: 1\right)$ yielded $3 \mathbf{e}(0.53 \mathrm{~g}, 77 \%)$ as white solid.

${ }^{1} \mathrm{H} \mathrm{NMR}\left(\mathrm{CDCl}_{3}, 600 \mathrm{MHz}\right): \delta 1.80(\mathrm{~s}, 3 \mathrm{H}), 3.55$ (t, $\left.1 \mathrm{H}, J=7.3 \mathrm{~Hz}\right)$, 3.61 (s, $3 \mathrm{H}), 3.65-3.74$ (m, $3 \mathrm{H}), 3.82$ (s, $3 \mathrm{H}), 4.77$ (d, 1H, J=13.1 Hz), 7.00 - 7.03 (m, 2 H), 7.07 - $7.11(\mathrm{~m}, 1 \mathrm{H}), 7.25$ - 7.30 (m, $4 \mathrm{H})$,

$7.42(\mathrm{~d}, 2 \mathrm{H}, J=6.6 \mathrm{~Hz}), 7.56(\mathrm{~d}, 1 \mathrm{H}, J=8.1 \mathrm{~Hz}) \mathrm{ppm} .{ }^{13} \mathrm{C}\{1 \mathrm{H}\} \mathrm{NMR}\left(\mathrm{CDCl}_{3}, 150 \mathrm{MHz}\right): \delta 18.0$, 45.7, 49.8, 50.8, 53.1, 81.3, 81.3, 83.9, 90.0, 125.2, 126.2, 126.2, 128.5 (2C), 128.6 (2C), 129.8 , 131.0, 136.2, 137.0, 141.1, 144.9, 153.6, 169.0 ppm. MS (ESI): m/z = 405 [M+H] $]^{+}$; HRMS (TOF $\left.\mathrm{ES}^{+}\right): \mathrm{m} / \mathrm{z}[\mathrm{M}+\mathrm{H}]^{+}$calculated for $\mathrm{C}_{24} \mathrm{H}_{24} \mathrm{~N}_{2} \mathrm{O}_{4}$ : 405.1808; found: 405.1809 . m.p. $121-122^{\circ} \mathrm{C}$. IR (KBr plate): 700, 1069, 1156, 1210, 1411, 1692, 1722, 1735, $2975 \mathrm{~cm}^{-1}$.

\section{Methyl (2E)-3-[2-(3-methoxy-3-oxoprop-1-yn-1-yl)-3-methyl-2-phenylimidazolidin-1-}

yl]prop-2-enoate (3f)

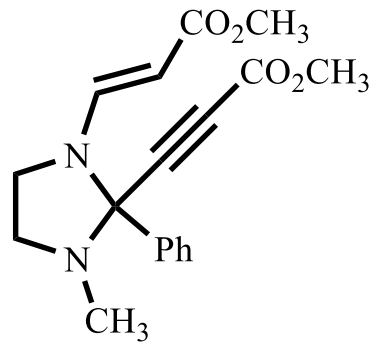

Following GP3 with 1-methyl-2-phenyl-4,5-dihydro-1 $H$-imidazole $1 f$

(1.7 mmol) after purification by $\mathrm{SiO}_{2}$ column chromatography $\left(\mathrm{CHCl}_{3}:\right.$ Hexane, $\left.1: 1\right)$ yielded $\mathbf{3 f}(0.54 \mathrm{~g}, 96 \%)$ as yellow oil.

${ }^{1} \mathrm{H}$ NMR $\left(\mathrm{CDCl}_{3}, 600 \mathrm{MHz}\right): \delta 2.19(\mathrm{~s}, 3 \mathrm{H}), 2.95(\mathrm{td}, 1 \mathrm{H}, J=9.3,8.1 \mathrm{~Hz})$, 3.32 - 3.40 (m, $2 \mathrm{H}), 3.51$ - 3.55 (m, $1 \mathrm{H}), 3.57$ (s, $3 \mathrm{H}), 3.82$ (s, $3 \mathrm{H}), 4.63$ $(\mathrm{d}, 1 \mathrm{H}, J=13.1 \mathrm{~Hz}), 7.09(\mathrm{~d}, 1 \mathrm{H}, J=13.1 \mathrm{~Hz}), 7.39-7.42(\mathrm{~m}, 3 \mathrm{H}), 7.61-7.64(\mathrm{~m}, 2 \mathrm{H}) \mathrm{ppm} .{ }^{13} \mathrm{C}$ $\{1 \mathrm{H}\} \mathrm{NMR}\left(\mathrm{CDCl}_{3}, 150 \mathrm{MHz}\right): \delta 34.8,45.5,50.7,51.0,53.1,81.1,81.5,82.8,89.2,128.4(2 \mathrm{C})$, 128.9 (2C), 130.2, 136.5, 145.0, 153.6, $169.1 \mathrm{ppm}$. MS (ESI): m/z = $329[\mathrm{M}+\mathrm{H}]^{+}$. HRMS (TOF $\left.\mathrm{ES}^{+}\right): \mathrm{m} / \mathrm{z}[\mathrm{M}+\mathrm{H}]^{+}$calculated for $\mathrm{C}_{18} \mathrm{H}_{20} \mathrm{~N}_{2} \mathrm{O}_{4}: 329.1495$; found: 329.1496. $\mathrm{R}_{\mathrm{f}}\left(\mathrm{CHCl}_{3}\right)=0.4$. IR (KBr plate): 1169, 1249, 1609, 1687, 1720, 2230, 2838, 2953, $3003 \mathrm{~cm}^{-1}$. 
Methyl (2E)-3-[3-benzyl-2-(3-methoxy-3-oxoprop-1-yn-1-yl)-2-phenylimidazolidin-1-

yl]prop-2-enoate (3g)

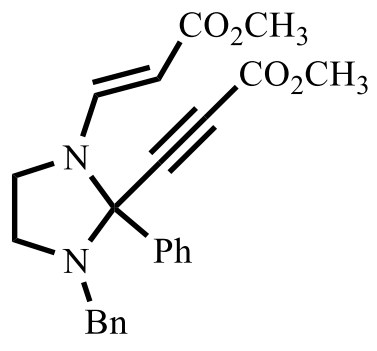

Following GP3 with 1-benzyl-2-phenyl-4,5-dihydro-1 $H$-imidazole $1 \mathrm{~g}$

(1.7 mmol) after purification by $\mathrm{SiO}_{2}$ column chromatography $\left(\mathrm{CHCl}_{3}:\right.$ Hexane, $\left.1: 1\right)$ yielded $3 \mathrm{~g}(0.62 \mathrm{~g}, 90 \%)$ as yellow solid.

${ }^{1} \mathrm{H}$ NMR $\left(\mathrm{CDCl}_{3}, 600 \mathrm{MHz}\right): \delta 2.85(\mathrm{dd}, 1 \mathrm{H}, J=17.7,8.1 \mathrm{~Hz}), 3.25(\mathrm{t}, 1 \mathrm{H}$, $J=8.1 \mathrm{~Hz}), 3.31(\mathrm{~d}, 1 \mathrm{H}, J=13.1 \mathrm{~Hz}), 3.37(\mathrm{t}, 1 \mathrm{H}, J=8.8 \mathrm{~Hz}), 3.47-3.51(\mathrm{~m}$,

$1 \mathrm{H}), 3.58$ (s, $3 \mathrm{H}), 3.61$ (d, 1H, J=13.1 Hz), $3.86(\mathrm{~s}, 3 \mathrm{H}), 4.65$ (d, 1H, J=13.1 Hz), 7.12 (d, 1H, $J=13.6 \mathrm{~Hz}), 7.20$ (d, 2H, J=6.6 Hz), $7.22-7.25$ (m, 1 H), $7.27-7.30$ (m, 2 H), $7.43-7.48$ (m, 3 $\mathrm{H}), 7.77$ - $7.80(\mathrm{~m}, 2 \mathrm{H}) \mathrm{ppm} .{ }^{13} \mathrm{C}\{1 \mathrm{H}\} \mathrm{NMR}\left(\mathrm{CDCl}_{3}, 150 \mathrm{MHz}\right): \delta 45.5,47.9,50.8,52.8,53.2$, 77.3, 81.4, 82.3, 89.3, 127.6, 128.5 (2C), 128.6 (2C), 128.7 (2C), 129.0 (2C), 130.4, 136.8, 137.4, 144.9, 153.6, 169.1 ppm. MS (ESI): $\mathrm{m} / \mathrm{z}=405[\mathrm{M}+\mathrm{H}]^{+}$. HRMS $\left(\mathrm{TOF} \mathrm{ES}^{+}\right): \mathrm{m} / \mathrm{z}[\mathrm{M}+\mathrm{H}]^{+}$calculated for $\mathrm{C}_{24} \mathrm{H}_{25} \mathrm{~N}_{2} \mathrm{O}_{4}$ : 405.1808; found: 405.1809. m.p. 120-121 ${ }^{\circ} \mathrm{C}$. IR ( $\mathrm{KBr}$ plate): 1150, 1256, 1614, $1699,1714,2230,2833,2947,3029 \mathrm{~cm}^{-1}$.

\section{Methyl (2E)-3-[3-benzyl-2-(3-methoxy-3-oxoprop-1-yn-1-yl)-2-(4-methoxyphenyl)}

\section{imidazolidin-1-yl]prop-2-enoate (3h)}

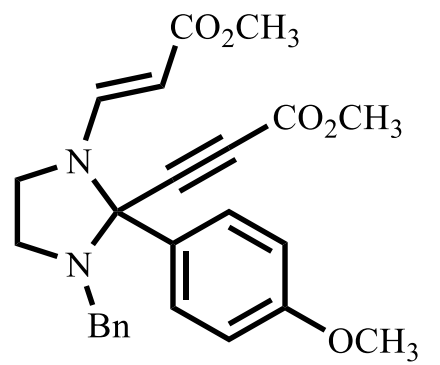

Following GP3 with 1-benzyl-2-(4-methoxyphenyl)-4,5-dihydro-1 $H$ imidazole $\mathbf{1 h}(1.7 \mathrm{mmol})$ after purification by $\mathrm{SiO}_{2}$ column chromatography $\left(\mathrm{CHCl}_{3}:\right.$ Hexane, $\left.1: 1\right)$ yielded $\mathbf{3 h}(0.71 \mathrm{~g}, 96 \%)$ as yellow solid.

${ }^{1} \mathrm{H}$ NMR $\left(\mathrm{CDCl}_{3}, 600 \mathrm{MHz}\right): \delta 2.82(\mathrm{q}, 1 \mathrm{H}, J=9.1 \mathrm{~Hz}), 3.24(\mathrm{t}, 1 \mathrm{H}$, $J=8.3 \mathrm{~Hz}$ ), $3.28(\mathrm{~d}, 1 \mathrm{H}, J=13.1 \mathrm{~Hz}), 3.35(\mathrm{t}, 1 \mathrm{H}, J=8.8 \mathrm{~Hz}), 3.46(\mathrm{q}, 1 \mathrm{H}, J=9.6 \mathrm{~Hz}), 3.59$ (s, $3 \mathrm{H})$, $3.62(\mathrm{~d}, 1 \mathrm{H}, J=13.1 \mathrm{~Hz}), 3.84$ (s, $3 \mathrm{H}), 3.86$ (s, $3 \mathrm{H}), 4.63$ (d, 1H, J=13.1 Hz), 6.96 (d, 2H, J=8.6 Hz), $7.13(\mathrm{~d}, 1 \mathrm{H}, J=13.1 \mathrm{~Hz}), 7.19-7.23$ (m, $2 \mathrm{H}), 7.24$ (d, 1H, J=7.1 Hz), 7.26 - 7.30 (m, $2 \mathrm{H})$, $7.70(\mathrm{~d}, 2 \mathrm{H}, J=9.1 \mathrm{~Hz}) \mathrm{ppm} .{ }^{13} \mathrm{C}\{1 \mathrm{H}\} \mathrm{NMR}\left(\mathrm{CDCl}_{3}, 150 \mathrm{MHz}\right): \delta 45.3,47.8,50.8,52.8,53.2$, 55.5, 81.1, 81.6, 82.1, 89.0, 114.4 (2C), 127.5, 128.4, 128.5 (2C), 128.7 (2C), 130.1 (2C), 137.5, 144.9, 153.7, 161.2, 169.2 ppm. MS (ESI): m/z = $457[\mathrm{M}+\mathrm{Na}]^{+}$. HRMS (TOF ES $\left.{ }^{+}\right): \mathrm{m} / \mathrm{z}[\mathrm{M}+\mathrm{H}]^{+}$ calculated for $\mathrm{C}_{25} \mathrm{H}_{26} \mathrm{~N}_{2} \mathrm{O}_{5}$ : 435.1914484; found: 435.1906. m.p. $114-115^{\circ} \mathrm{C}$. IR (KBr plate): 1028 , $1161,1252,1322,1360,1454,1511,1612,1692$, 1720, 2226, 2814, 2840, 2947, 3002, 3029, 3370, $3416 \mathrm{~cm}^{-1}$. 
Methyl (2E)-3-[3-benzyl-2-(4-chlorophenyl)-2-(3-methoxy-3-oxoprop-1-yn-1-yl)

\section{imidazolidin-1-yl]prop-2-enoate (3i)}

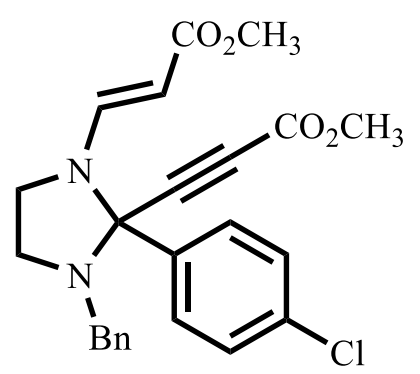

Following GP3 with 1-benzyl-2-(4-chlorophenyl)-4,5-dihydro-1Himidazole $1 \mathbf{1}(1.7 \mathrm{mmol})$ after purification by $\mathrm{SiO}_{2}$ column chromatography $\left(\mathrm{CHCl}_{3}: \mathrm{Hexane}, 1: 1\right)$ yielded $3 \mathbf{i}(0.67 \mathrm{~g}, 90 \%)$ as yellow solid.

${ }^{1} \mathrm{H} \mathrm{NMR}\left(\mathrm{CDCl}_{3}, 600 \mathrm{MHz}\right): \delta 2.82(\mathrm{q}, 1 \mathrm{H}, J=8.8 \mathrm{~Hz}), 3.24$ (t, $1 \mathrm{H}, J=8.3$ Hz), 3.30 (d, 1H, J=12.6 Hz), 3.35 (t, 1H, J=8.8 Hz), $3.44-3.49$ (m, 1 H), 3.56 (d, 1H, J=13.1 Hz), 3.58 (s, $3 \mathrm{H}), 3.85$ (s, $3 \mathrm{H}), 4.64$ (d, 1H, J=13.1 Hz), 7.07 (d, 1H, $J=13.1 \mathrm{~Hz}), 7.16(\mathrm{~d}, 2 \mathrm{H}, J=7.1 \mathrm{~Hz}), 7.21-7.28(\mathrm{~m}, 3 \mathrm{H}), 7.41(\mathrm{dd}, 2 \mathrm{H}, J=8.6,2.0 \mathrm{~Hz}), 7.70(\mathrm{~d}$, $2 \mathrm{H}, J=6.6 \mathrm{~Hz}) \mathrm{ppm} .{ }^{13} \mathrm{C}\{1 \mathrm{H}\} \mathrm{NMR}\left(\mathrm{CDCl}_{3}, 150 \mathrm{MHz}\right): \delta 45.5,48.0,50.8,52.9,53.2,80.7,81.6$, 81.8, 89.9, 127.7, 128.6 (2C), 128.6 (2C), 129.3 (2C), 130.1 (2C), 135.6, 136.5, 137.1, 144.5, 153.5, 168.9 ppm. MS (ESI): m/z = $439[\mathrm{M}+\mathrm{H}]^{+}$. HRMS $\left(\mathrm{TOF} \mathrm{ES}^{+}\right): \mathrm{m} / \mathrm{z}[\mathrm{M}+\mathrm{H}]^{+}$calculated for $\mathrm{C}_{24} \mathrm{H}_{23} \mathrm{ClN}_{2} \mathrm{O}_{4}$ : 439.1419; found: 439.1413. m.p. $143-144^{\circ} \mathrm{C}$. IR (KBr plate): 702, 814, 965, 1091, $1157,1249,1486,1616,1701,1712,1724,2227,2818,2951,2987 \mathrm{~cm}^{-1}$.

\section{Methyl (2E)-3-[3-benzyl-2-(3-methoxy-3-oxoprop-1-yn-1-yl)-2-(4-nitrophenyl)imidazolidin-}

1-yl]prop-2-enoate $(3 \mathbf{j})$

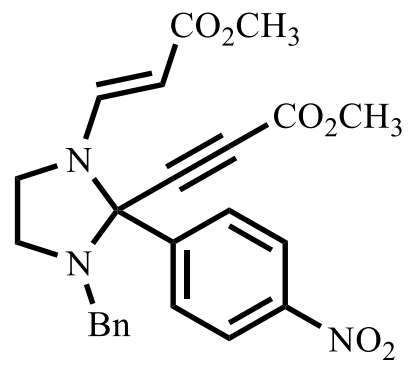

Following GP3 with 1-benzyl-2-(4-nitrophenyl)-4,5-dihydro-1 $H$ imidazole $\mathbf{1 j}(1.7 \mathrm{mmol})$ after purification by $\mathrm{SiO}_{2}$ column chromatography $\left(\mathrm{CHCl}_{3}:\right.$ Hexane, 1:1) yielded $\mathbf{3 j}(0.69 \mathrm{~g}, 90 \%)$ as yellow oil.

${ }^{1} \mathrm{H}$ NMR $\left(\mathrm{CDCl}_{3}, 600 \mathrm{MHz}\right): \delta 2.86-2.93(\mathrm{~m}, 1 \mathrm{H}), 3.31(\mathrm{t}, 1 \mathrm{H}, J=8.1$ Hz), 3.38 - 3.44 (m, 2 H), 3.53 - 3.57 (m, 2 H), 3.58 (s, 3 H), 3.88 (s, 3 H), 4.69 (d, 1H, J=13.1 Hz), $7.03(\mathrm{~d}, 1 \mathrm{H}, J=13.1 \mathrm{~Hz}), 7.15(\mathrm{~d}, 2 \mathrm{H}, J=6.6 \mathrm{~Hz}), 7.21-7.28(\mathrm{~m}, 3 \mathrm{H}), 7.96(\mathrm{dt}, 2 \mathrm{H}, J=13.6$, $2.0 \mathrm{~Hz}), 8.28$ (dt, $2 \mathrm{H}, J=14.1,2.5 \mathrm{~Hz}) \mathrm{ppm} .{ }^{13} \mathrm{C}\{1 \mathrm{H}\} \mathrm{NMR}\left(\mathrm{CDCl}_{3}, 150 \mathrm{MHz}\right): \delta 45.9,48.4,50.9$, 53.1, 53.3, 79.7, 81.4, 82.3, 90.9, 124.1 (2C), 127.8 (2C), 128.6 (2C), 128.8, 129.8 (2C), 136.5, 144.0, 144.4, 149.2, 153.2, 168.7 ppm. MS (ESI): m/z = $451[\mathrm{M}+\mathrm{H}]^{+}$. HRMS $\left(\mathrm{TOF} \mathrm{ES}^{+}\right): \mathrm{m} / \mathrm{z}$ $[\mathrm{M}+\mathrm{H}]^{+}$calculated for $\mathrm{C}_{24} \mathrm{H}_{23} \mathrm{~N}_{3} \mathrm{O}_{6}$ : 450.1659; found: 450.1660. $\mathrm{R}_{\mathrm{f}}\left(\mathrm{CHCl}_{3}\right)=0.4$. IR ( $\mathrm{KBr}$ plate): 1151, 1244, 1519, 1610, 1714, 2227, 2838, $2948 \mathrm{~cm}^{-1}$. 


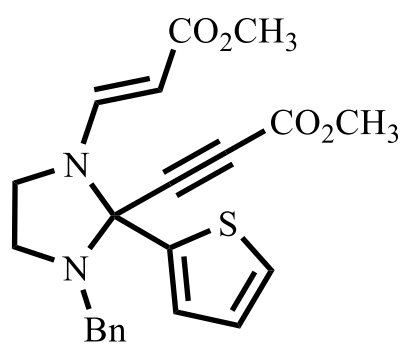

Following GP3 with 1-benzyl-2-(thiophen-2-yl)-4,5-dihydro-1 $H$ imidazole $1 \mathbf{k}(1.7 \mathrm{mmol})$ after purification by $\mathrm{SiO}_{2}$ column chromatography $\left(\mathrm{CHCl}_{3}: \mathrm{Hexane}, 1: 1\right)$ yielded $\mathbf{3 k}(0.57 \mathrm{~g}, 82 \%)$ as yellow solid.

${ }^{1} \mathrm{H} \mathrm{NMR}\left(\mathrm{CDCl}_{3}, 600 \mathrm{MHz}\right): \delta 2.80(\mathrm{td}, 1 \mathrm{H}, J=17.7,8.1 \mathrm{~Hz}), 3.21(\mathrm{t}$, $1 \mathrm{H}, J=8.1 \mathrm{~Hz}), 3.32$ (t, 1H, J=8.6 Hz), 3.35 (d, 1H, $J=12.6 \mathrm{~Hz}), 3.42$ (td, $1 \mathrm{H}, J=17.2,7.6 \mathrm{~Hz}), 3.61$ $(\mathrm{s}, 3 \mathrm{H}), 3.85(\mathrm{~s}, 3 \mathrm{H}), 3.87$ (d, 1H, J=13.1 Hz), 4.66 (d, 1H, J=13.1 Hz), 7.02 (dd, 1H, J=5.3, 3.8 Hz), $7.25-7.29(\mathrm{~m}, 1 \mathrm{H}), 7.29-7.36(\mathrm{~m}, 5 \mathrm{H}), 7.46$ (dd, 1H, J=5.3, $1.3 \mathrm{~Hz}), 7.62(\mathrm{dd}, 1 \mathrm{H}, J=3.5$, $1.5 \mathrm{~Hz}) \mathrm{ppm} .{ }^{13} \mathrm{C}\{1 \mathrm{H}\} \mathrm{NMR}\left(\mathrm{CDCl}_{3}, 150 \mathrm{MHz}\right): \delta 44.8,47.6,50.8,53.2,53.3,78.8,79.5,81.0$, 89.5, 126.8, 127.7, 128.6 (2C), 128.7 (2C), 129.5, 131.1, 137.2, 142.2, 144.2, 153.4, 169.6 ppm. MS (ESI): $\mathrm{m} / \mathrm{z}=411[\mathrm{M}+\mathrm{H}]^{+}$. HRMS $\left(\right.$TOF ES $\left.{ }^{+}\right): \mathrm{m} / \mathrm{z}[\mathrm{M}+\mathrm{H}]^{+}$calculated for $\mathrm{C}_{22} \mathrm{H}_{22} \mathrm{~N}_{2} \mathrm{O}_{4} \mathrm{~S}$ : 411.1373; found: 411.1373. m.p. $140-141^{\circ} \mathrm{C}$. IR (KBr plate): 706, 811, 1149, 1253, 1322, 1436, $1612,1697,1712,2229,2841,2946 \mathrm{~cm}^{-1}$.

\section{Methyl (2E)-3-[3-benzyl-2-(3-methoxy-3-oxoprop-1-yn-1-yl)-2-methylimidazolidin-1-} yl]prop-2-enoate (31)

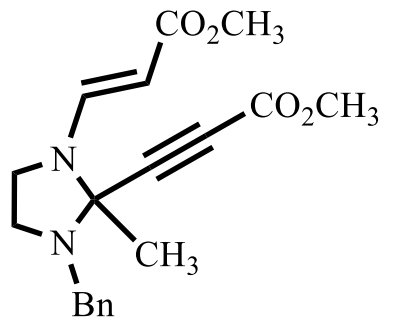

Following GP3 with 1-benzyl-2-methyl-4,5-dihydro- $1 H$-imidazole 11 $(1.7 \mathrm{mmol})$ after purification by $\mathrm{SiO}_{2}$ column chromatography $\left(\mathrm{CHCl}_{3}:\right.$ Hexane, $\left.1: 1\right)$ yielded $31(0.37 \mathrm{~g}, 63 \%)$ as yellow solid.

${ }^{1} \mathrm{H} \mathrm{NMR}\left(\mathrm{CDCl}_{3}, 600 \mathrm{MHz}\right): \delta 1.74(\mathrm{~s}, 3 \mathrm{H}), 2.65(\mathrm{td}, 1 \mathrm{H}, J=9.3,8.1 \mathrm{~Hz})$, 3.08 (ddd, 1H, J=9.1, 7.3, $1.3 \mathrm{~Hz}), 3.17$ - 3.28 (m, $2 \mathrm{H}), 3.41(\mathrm{~d}, 1 \mathrm{H}$, $J=13.1 \mathrm{~Hz}), 3.69$ (s, $3 \mathrm{H}), 3.80(\mathrm{~s}, 3 \mathrm{H}), 4.00$ (d, 1H, J=13.1 Hz), 4.66 (d, 1H, J=13.1 Hz), 7.26 $7.30(\mathrm{~m}, 1 \mathrm{H}), 7.32-7.36(\mathrm{~m}, 4 \mathrm{H}), 7.67(\mathrm{~d}, 1 \mathrm{H}, J=13.1 \mathrm{~Hz}) \mathrm{ppm} .{ }^{13} \mathrm{C}\{1 \mathrm{H}\} \mathrm{NMR}\left(\mathrm{CDCl}_{3}, 150\right.$ MHz): $\delta 25.4,44.9,48.4,50.9,53.1,53.8,75.7,77.8,83.5,88.8,127.7$ (2C), 128.6, 128.7 (2C), 137.5, 143.8, 153.6, 169.4 ppm. MS (ESI): m/z = $343[\mathrm{M}+\mathrm{H}]^{+}$. HRMS (TOF ES ${ }^{+}$): m/z $[\mathrm{M}+\mathrm{H}]^{+}$ calculated for $\mathrm{C}_{19} \mathrm{H}_{22} \mathrm{~N}_{2} \mathrm{O}_{4}$ : 343.1652; found: 343.1648. m.p. $111-112^{\circ} \mathrm{C}$. IR (KBr plate): 745 , $751,1162,1323,1430,1608,1684,1713,2223,2820,2952 \mathrm{~cm}^{-1}$. 
(3E)-4-[3-Phenyl-2-(3-oxobut-1-yn-1-yl)-2-phenylimidazolidin-1-yl]but-3-en-2-one (3m)

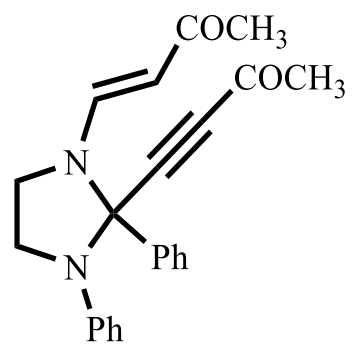

Following GP3 with 1,2-diphenyl-4,5-dihydro- $H H$-imidazole 1a (1.7 mmol) after purification by $\mathrm{SiO}_{2}$ column chromatography $\left(\mathrm{CHCl}_{3}: \mathrm{Hexane}\right.$, 1:1) yielded $3 \mathbf{m}(0.41 \mathrm{~g}, 67 \%)$ as orange oil.

${ }^{1} \mathrm{H}$ NMR $\left(\mathrm{CDCl}_{3}, 600 \mathrm{MHz}\right): \delta 2.01(\mathrm{~s}, 3 \mathrm{H}), 2.28(\mathrm{~s}, 3 \mathrm{H}), 3.63-3.66(\mathrm{~m}$, $1 \mathrm{H}), 3.71-3.75(\mathrm{~m}, 2 \mathrm{H}), 4.08-4.12(\mathrm{~m}, 1 \mathrm{H}), 5.21(\mathrm{~d}, 1 \mathrm{H}, J=13.1 \mathrm{~Hz})$, $6.62(\mathrm{dd}, 2 \mathrm{H}, J=9.1,1.0 \mathrm{~Hz}), 6.84-6.87(\mathrm{~m}, 1 \mathrm{H}), 7.10-7.16(\mathrm{~m}, 3 \mathrm{H}), 7.43-7.45(\mathrm{~m}, 3 \mathrm{H}), 7.68$ $-7.70(\mathrm{~m}, 2 \mathrm{H}) \mathrm{ppm} .{ }^{13} \mathrm{C}\{1 \mathrm{H}\} \mathrm{NMR}\left(\mathrm{CDCl}_{3}, 150 \mathrm{MHz}\right): \delta 26.6,32.0,43.5,45.8,79.0,85.0,85.3$, 101.8, 111.7, 116.0 (2C), 119.9, 126.3 (2C), 128.8 (2C), 128.2, 128.4 (2C), 129.2, 142.7, 182.5, 187.6 ppm. HRMS (TOF $\mathrm{ES}^{+}$): $\mathrm{m} / \mathrm{z}[\mathrm{M}+\mathrm{H}]^{+}$calculated for $\mathrm{C}_{23} \mathrm{H}_{22} \mathrm{~N}_{2} \mathrm{O}_{2}$ : 359.1754; found: 359.1751. $\mathrm{R}_{\mathrm{f}}\left(\mathrm{CHCl}_{3}: \mathrm{MeOH}, 100: 1\right)=0.32 . \mathrm{IR}$ (KBr plate): 702, 811, 1350, 1381, 1630, 1715, 2882 $\mathrm{cm}^{-1}$.

(3E)-4-[3-(4-Chlorophenyl)-2-(3-oxobut-1-yn-1-yl)-2-phenylimidazolidin-1-yl]but-3-en-2one $(3 n)$

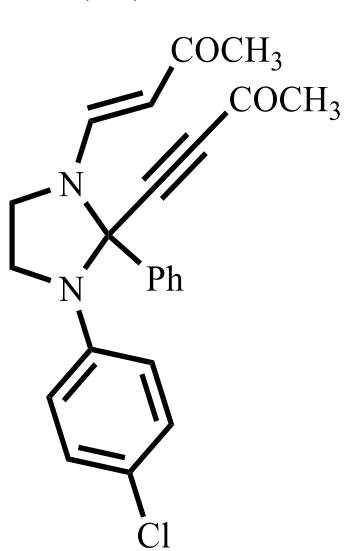

Following GP3 with 1-(4-chlorophenyl)-2-phenyl-4,5-dihydro-1 $\mathrm{H}$ imidazole 1c $(1.7 \mathrm{mmol})$ after purification by $\mathrm{SiO}_{2}$ column chromatography $\left(\mathrm{CHCl}_{3}:\right.$ Hexane, $\left.1: 1\right)$ yielded $3 \mathrm{n}(0.43 \mathrm{~g}, 65 \%)$ as red oil.

${ }^{1} \mathrm{H}$ NMR $\left(\mathrm{CDCl}_{3}, 600 \mathrm{MHz}\right): \delta 2.01(\mathrm{~s}, 3 \mathrm{H}), 2.31(\mathrm{~s}, 3 \mathrm{H}), 3.61-3.66(\mathrm{~m}$, $1 \mathrm{H}), 3.67$ - $3.76(\mathrm{~m}, 2 \mathrm{H}), 4.05(\mathrm{td}, 1 \mathrm{H}, J=7.7,3.3 \mathrm{~Hz}), 5.21$ (d, 1H, $J=13.1$ $\mathrm{Hz}), 6.53$ (d, 2H, J=9.1 Hz), 7.06 (d, 2H, J=9.1 Hz), 7.12 (d, 1H, J=13.1 $\mathrm{Hz}), 7.43-7.46(\mathrm{~m}, 3 \mathrm{H}), 7.64-7.67(\mathrm{~m}, 2 \mathrm{H}) \mathrm{ppm} .{ }^{13} \mathrm{C}\{1 \mathrm{H}\} \mathrm{NMR}$ $\left(\mathrm{CDCl}_{3}, 150 \mathrm{MHz}\right): \delta 27.8,33.1,44.6,47.1,80.1,85.4,86.5,103.2,118.3$ (2C), 126.2 (2C), 127.5, 128.8 (2C), 129.6 (2C), 130.5, 137.9, 141.9, 143.6, 183.4, 195.8 ppm. MS (ESI): $\mathrm{m} / \mathrm{z}=393[\mathrm{M}+\mathrm{H}]^{+}$. HRMS $\left(\mathrm{TOF} \mathrm{ES}^{+}\right): \mathrm{m} / \mathrm{z}[\mathrm{M}+\mathrm{H}]^{+}$calculated for $\mathrm{C}_{23} \mathrm{H}_{21} \mathrm{ClN}_{2} \mathrm{O}_{2}$ : 393.1364; found: 393.1360. $\mathrm{R}_{\mathrm{f}}\left(\mathrm{CHCl}_{3}: \mathrm{MeOH}, 100: 1\right)=0.30$. IR ( $\mathrm{KBr}$ plate): 700, 810, 1349, $1378,1624,1710,2880 \mathrm{~cm}^{-1}$. 
(3E)-4-[3-Methyl-2-(3-oxobut-1-yn-1-yl)-2-phenylimidazolidin-1-yl]but-3-en-2-one (3o)

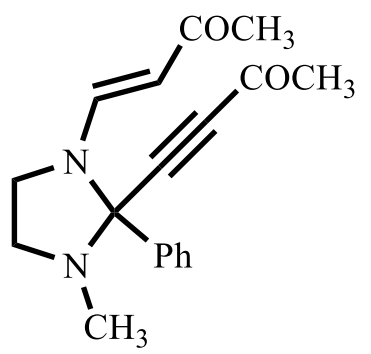

Following GP3 with 1-methyl-2-phenyl-4,5-dihydro-1 $H$-imidazole $1 \mathbf{f}$ (1.7 mmol) after purification by $\mathrm{SiO}_{2}$ column chromatography $\left(\mathrm{CHCl}_{3}:\right.$ Hexane, $\left.1: 1\right)$ yielded $3 \mathrm{o}(0.32 \mathrm{~g}, 64 \%)$ as brown solid.

${ }^{1} \mathrm{H} \mathrm{NMR}\left(\mathrm{CDCl}_{3}, 600 \mathrm{MHz}\right): \delta 1.97$ (s, $\left.3 \mathrm{H}\right), 2.18$ (s, $\left.3 \mathrm{H}\right), 2.45$ (s, $\left.3 \mathrm{H}\right)$, 2.90 - $2.97(\mathrm{~m}, 1 \mathrm{H}), 3.34$ - $3.42(\mathrm{~m}, 2 \mathrm{H}), 3.51$ - 3.60 (m, $1 \mathrm{H}), 5.11(\mathrm{~d}$, $1 \mathrm{H}, J=13.1 \mathrm{~Hz}), 7.07(\mathrm{~d}, 1 \mathrm{H}, J=13.1 \mathrm{~Hz}), 7.39-7.44(\mathrm{~m}, 3 \mathrm{H}), 7.61(\mathrm{dd}, 2 \mathrm{H}, J=6.8,2.8 \mathrm{~Hz}) \mathrm{ppm}$. ${ }^{13} \mathrm{C}\{1 \mathrm{H}\} \mathrm{NMR}\left(\mathrm{CDCl}_{3}, 150 \mathrm{MHz}\right): \delta 27.8,33.3,34.8,45.6,51.0,83.0,84.2,88.9,101.4,128.3$ (2C), 129.0 (2C), 130.4, 136.5, 144.5, 183.7, 195.6 ppm. MS (ESI): m/z = $297[\mathrm{M}+\mathrm{H}]^{+}$. HRMS (TOF ES ${ }^{+}$): $\mathrm{m} / \mathrm{z}[\mathrm{M}+\mathrm{H}]^{+}$calculated for $\mathrm{C}_{18} \mathrm{H}_{20} \mathrm{~N}_{2} \mathrm{O}_{2}$ : 297.1597; found: 297.1594. m.p. 114-115 ${ }^{\circ} \mathrm{C}$. IR (KBr plate): 1175, 1330, 1568, 1672, 2196, 2802, $2838 \mathrm{~cm}^{-1}$.

(3E)-4-[3-Benzyl-2-(3-oxobut-1-yn-1-yl)-2-phenylimidazolidin-1-yl]but-3-en-2-one (3p)

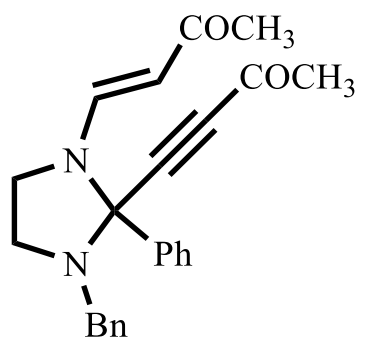

Following GP3 with 1-benzyl-2-phenyl-4,5-dihydro-1H-imidazole $\mathbf{1 g}$ $(1.7 \mathrm{mmol})$ after purification by $\mathrm{SiO}_{2}$ column chromatography $\left(\mathrm{CHCl}_{3}:\right.$ Hexane, $\left.2: 1\right)$ yielded $\mathbf{3 p}(0.38 \mathrm{~g}, 60 \%)$ as yellow solid.

${ }^{1} \mathrm{H} \mathrm{NMR}\left(\mathrm{CDCl}_{3}, 600 \mathrm{MHz}\right): \delta 1.99$ (s, $\left.3 \mathrm{H}\right), 2.51$ (s, $\left.3 \mathrm{H}\right), 2.83(\mathrm{td}, 1 \mathrm{H}$, $J=9.3,8.1 \mathrm{~Hz}), 3.26(\mathrm{t}, 1 \mathrm{H}, J=7.6 \mathrm{~Hz}), 3.28(\mathrm{~d}, 1 \mathrm{H}, J=12.6 \mathrm{~Hz}) 3.41(\mathrm{t}$, $1 \mathrm{H}, J=8.8 \mathrm{~Hz}), 3.48-3.54(\mathrm{~m}, 1 \mathrm{H}), 3.61(\mathrm{~d}, 1 \mathrm{H}, J=12.6 \mathrm{~Hz}), 5.12(\mathrm{~d}, 1 \mathrm{H}, J=13.1 \mathrm{~Hz}), 7.10(\mathrm{~d}$, $1 \mathrm{H}, J=13.1 \mathrm{~Hz}), 7.19(\mathrm{~d}, 2 \mathrm{H}, J=6.6 \mathrm{~Hz}), 7.22-7.25$ (m, $1 \mathrm{H}), 7.26$ - 7.30 (m, $2 \mathrm{H}), 7.44$ - 7.49 (m, $3 \mathrm{H}), 7.77(\mathrm{dd}, 2 \mathrm{H}, J=7.6,2.0 \mathrm{~Hz}) \mathrm{ppm} .{ }^{13} \mathrm{C}\{1 \mathrm{H}\} \mathrm{NMR}\left(\mathrm{CDCl}_{3}, 150 \mathrm{MHz}\right): \delta 27.9,33.4,45.7$, 47.9, 52.9, 82.6, 84.5, 88.8, 101.4, 127.6, 128.6 (2C), 128.6 (2C), 128.7 (2C), 129.1 (2C), 130.6, 136.8, 137.2, 144.4, 183.7, 195.7 ppm. MS (ESI): m/z = $373[\mathrm{M}+\mathrm{H}]^{+}$. HRMS $\left(\mathrm{TOF}^{\mathrm{E}} \mathrm{S}^{+}\right): \mathrm{m} / \mathrm{z}$ $[\mathrm{M}+\mathrm{H}]^{+}$calculated for $\mathrm{C}_{24} \mathrm{H}_{24} \mathrm{~N}_{2} \mathrm{O}_{2}$ : 373.1910; found: 373.1911. m.p. 114-115 ${ }^{\circ} \mathrm{C}$. IR (KBr plate): $1169,1326,1573,1667,2198,2899 \mathrm{~cm}^{-1}$. 
(3E)-4-[3-Benzyl-2-(4-chlorophenyl)-2-(3-oxobut-1-yn-1-yl)imidazolidin-1-yl]but-3-en-2one $(3 q)$

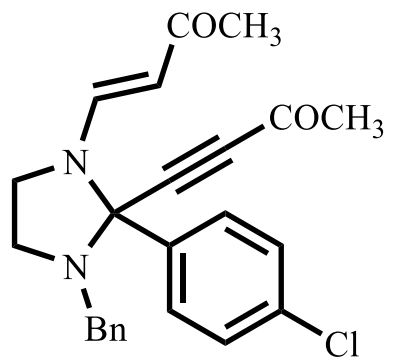

Following GP3 with 1-benzyl-2-(4-chlorophenyl)-4,5-dihydro-1 $\mathrm{H}$ imidazole $1 \mathbf{1 i}(1.7 \mathrm{mmol})$ after purification by $\mathrm{SiO}_{2}$ column chromatography $\left(\mathrm{CHCl}_{3}: H e x a n e, 2: 1\right)$ yielded $3 \mathbf{q}(0.47 \mathrm{~g}, 68 \%)$ as yellow solid.

${ }^{1} \mathrm{H}$ NMR $\left(\mathrm{CDCl}_{3}, 600 \mathrm{MHz}\right): \delta 1.99$ (s, $\left.3 \mathrm{H}\right), 2.48$ (s, $\left.3 \mathrm{H}\right), 2.80(\mathrm{td}, 1 \mathrm{H}$, $J=17.7,8.1 \mathrm{~Hz}), 3.24(\mathrm{t}, 1 \mathrm{H}, J=8.1 \mathrm{~Hz}), 3.27(\mathrm{t}, 1 \mathrm{H}, J=13.1 \mathrm{~Hz}), 3.38$ (t, 1H, $J=8.8 \mathrm{~Hz}), 3.48$ (td, $1 \mathrm{H}, J=17.2,7.6 \mathrm{~Hz}), 3.55(\mathrm{~d}, 1 \mathrm{H}, J=12.6 \mathrm{~Hz}), 5.11(\mathrm{~d}, 1 \mathrm{H}, J=13.1 \mathrm{~Hz}), 7.06(\mathrm{~d}, 1 \mathrm{H}, J=13.1 \mathrm{~Hz})$, $7.15(\mathrm{~d}, 2 \mathrm{H}, J=6.6 \mathrm{~Hz}), 7.22-7.27(\mathrm{~m}, 3 \mathrm{H}), 7.42$ (d, 2H, J=8.6 Hz), 7.68 (d, 2H, J=8.6 Hz) ppm. ${ }^{13} \mathrm{C}\{1 \mathrm{H}\} \mathrm{NMR}\left(\mathrm{CDCl}_{3}, 150 \mathrm{MHz}\right): \delta 28.2,33.4,45.7,47.9,52.9,82.0,83.7,89.0,101.6,127.8$, 128.6 (4C), 129.4 (2C), 130.0 (2C), 135.6, 136.6, 136.9, 143.9, 183.6, 195.7 ppm. MS (ESI): m/z $=407[\mathrm{M}+\mathrm{H}]^{+}$. HRMS $\left(\right.$TOF ES $\left.{ }^{+}\right): \mathrm{m} / \mathrm{z}[\mathrm{M}+\mathrm{H}]^{+}$calculated for $\mathrm{C}_{24} \mathrm{H}_{23} \mathrm{ClN}_{2} \mathrm{O}_{2}: 407.1520$; found: 407.1514. m.p. $132-133^{\circ} \mathrm{C}$. IR (KBr plate): 964, 1169, 1320, 1569, 1663, 1684, 2203, $2876 \mathrm{~cm}^{-1}$.

(3E)-4-[3-Benzyl-2-(3-oxobut-1-yn-1-yl)-2-(thiophen-2-yl)imidazolidin-1-yl]but-3-en-2-one (3r)

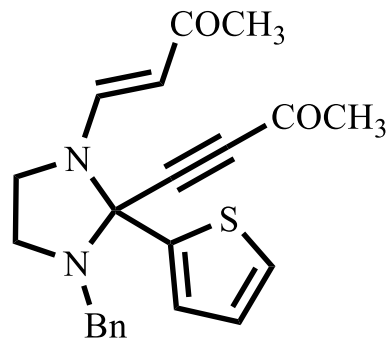

Following GP3 with 1-benzyl-2-(thiophen-2-yl)-4,5-dihydro- $1 H$ imidazole $1 \mathbf{k}(1.7 \mathrm{mmol})$ after purification by $\mathrm{SiO}_{2}$ column chromatography $\left(\mathrm{CHCl}_{3}: H e x a n e, 2: 1\right)$ yielded $3 \mathbf{r}(0.44 \mathrm{~g}, 69 \%)$ as yellow solid.

${ }^{1} \mathrm{H}$ NMR $\left(\mathrm{CDCl}_{3}, 600 \mathrm{MHz}\right): \delta 2.02(\mathrm{~s}, 3 \mathrm{H}), 2.48$ (s, $\left.3 \mathrm{H}\right), 2.77(\mathrm{td}, 1 \mathrm{H}$, $J=17.2,7.6 \mathrm{~Hz}), 3.21(\mathrm{t}, 1 \mathrm{H}, J=8.6 \mathrm{~Hz}), 3.32(\mathrm{~d}, 1 \mathrm{H}, J=12.6 \mathrm{~Hz}), 3.34$ (t, $1 \mathrm{H}, J=8.6 \mathrm{~Hz}), 3.43(\mathrm{td}$, 1H, $J=17.2,7.6 \mathrm{~Hz}), 3.86(\mathrm{~d}, 1 \mathrm{H}, J=13.1 \mathrm{~Hz}), 5.13$ (d, 1H, $J=13.6 \mathrm{~Hz}), 7.03$ (dd, 1H, J=5.0, 3.5 Hz), 7.24 - 7.28 (m, 2 H), 7.29 - 7.34 (m, 4 H), 7.46 (dd, 1H, J=5.0, $1.0 \mathrm{~Hz}), 7.58$ (dd, 1H, J=3.8, $1.3 \mathrm{~Hz}) \mathrm{ppm} .{ }^{13} \mathrm{C}\{1 \mathrm{H}\} \mathrm{NMR}\left(\mathrm{CDCl}_{3}, 150 \mathrm{MHz}\right): \delta 28.0,33.3,44.9,47.5,53.3,79.0,84.0,86.8$, 101.4, 126.8, 127.7, 128.6 (2C), 128.7 (2C), 129.6, 130.9, 137.0, 142.2, 143.7, 183.6, 195.7 ppm. MS (ESI): $\mathrm{m} / \mathrm{z}=379[\mathrm{M}+\mathrm{H}]^{+}$. HRMS $\left(\right.$TOF ES $\left.{ }^{+}\right): \mathrm{m} / \mathrm{z}[\mathrm{M}+\mathrm{H}]^{+}$calculated for $\mathrm{C}_{22} \mathrm{H}_{22} \mathrm{~N}_{2} \mathrm{O}_{2} \mathrm{~S}$ : 379.1475; found: 379.1474 . m.p. $132-133^{\circ} \mathrm{C}$. IR (KBr plate): 1165, 1326, 1573, 1667, 2199, 2830 , $3113 \mathrm{~cm}^{-1}$. 
4.4 Dimethyl 2-benzoyl-1-[2-(arylamino)ethyl]-1 $H$-pyrrole-3,4-dicarboxylates 4:

Dimethyl 2-benzoyl-1-[2-(phenylamino)ethyl]-1H-pyrrole-3,4-dicarboxylate (4a)

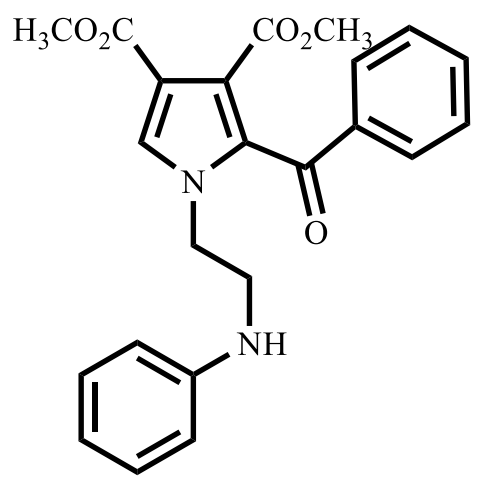

Following GP4 with methyl (2E)-3-[2-(3-methoxy-3-oxoprop-1-

yn-1-yl)-2,3-diphenylimidazolidin-1-yl]prop-2-enoate $\quad 3 \mathbf{a} \quad(0.5$ mmol) after purification by $\mathrm{SiO}_{2}$ column chromatography $\left(\mathrm{CHCl}_{3}:\right.$ Hexane, $\left.1: 1\right)$ yielded $4 \mathbf{a}(158 \mathrm{mg}, 78 \%)$ as white solid.

Following GP5 with 1,2-diphenyl-4,5-dihydro- $1 H$-imidazole 1a (2.25 mmol) yielded $\mathbf{4 a}(630 \mathrm{mg}, 69 \%)$.

${ }^{1} \mathrm{H} \mathrm{NMR}\left(\mathrm{CDCl}_{3}, 600 \mathrm{MHz}\right): \delta 3.21(\mathrm{~s}, 3 \mathrm{H}), 3.58(\mathrm{t}, 2 \mathrm{H}, J=5.8 \mathrm{~Hz})$, 3.79 (s, $3 \mathrm{H}), 4.00$ (br., $1 \mathrm{H}), 4.38$ (t, 2H, J=5.5 Hz), 6.53 (d, 2H, J=7.6 Hz), 6.72 (t, 1H, J=7.3 Hz), $7.15(\mathrm{t}, 2 \mathrm{H}, J=7.8 \mathrm{~Hz}), 7.39-7.45(\mathrm{~m}, 3 \mathrm{H}), 7.53-7.58(\mathrm{~m}, 1 \mathrm{H}), 7.71(\mathrm{dd}, 2 \mathrm{H}, J=8.1,1.5$ $\mathrm{Hz}) \mathrm{ppm} .{ }^{13} \mathrm{C}\{1 \mathrm{H}\} \mathrm{NMR}\left(\mathrm{CDCl}_{3}, 150 \mathrm{MHz}\right): \delta 45.0,48.5,51.8,51.9,112.8(2 \mathrm{C}), 114.4,118.1$, 124.0, 128.5 (2C), 129.1 (2C), 129.5 (2C), 131.0, 131.8, 133.1, 138.6, 147.0, 163.0, 164.4, 187.9 ppm. MS (ESI): $\mathrm{m} / \mathrm{z}=429[\mathrm{M}+\mathrm{Na}]^{+}$. HRMS $\left(\mathrm{TOF} \mathrm{ES}^{+}\right): \mathrm{m} / \mathrm{z}[\mathrm{M}+\mathrm{H}]^{+}$calculated for $\mathrm{C}_{23} \mathrm{H}_{22} \mathrm{~N}_{2} \mathrm{O}_{5}$ : 407.1601; found: 407.1612. m.p. $150-151^{\circ} \mathrm{C}$. IR (KBr plate): 1219, 1267, 1399, 1514, 1632, 1713, $1733,2947,3375 \mathrm{~cm}^{-1}$.

Dimethyl 2-benzoyl-1-\{2-[(4-methoxyphenyl)amino]ethyl $\}-1 H$-pyrrole-3,4-dicarboxylate (4b)

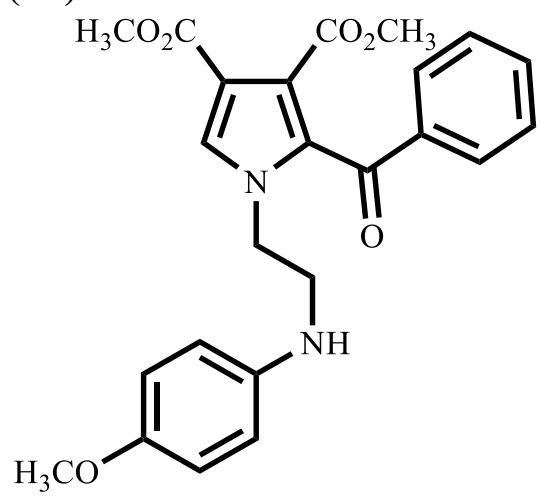

Following GP4 with methyl (2E)-3-[2-(3-methoxy-3-oxoprop1-yn-1-yl)-3-(4-methoxyphenyl)-2-phenylimidazolidin-1-yl] prop -2-enoate $\mathbf{3 b}(0.5 \mathrm{mmol})$ after purification by $\mathrm{SiO}_{2}$ column chromatography $\left(\mathrm{CHCl}_{3}: H e x a n e, 1: 1\right)$ yielded $\mathbf{4 b}(124 \mathrm{mg}$, $57 \%$ ) as yellow solid.

Following GP5 with 1-(4-methoxyphenyl)-2-phenyl-4,5dihydro- $1 H$-imidazole $1 \mathbf{b}(1.1 \mathrm{mmol})$ yielded $4 \mathbf{b}(254 \mathrm{mg}, 53 \%)$. ${ }^{1} \mathrm{H} \mathrm{NMR}\left(\mathrm{CDCl}_{3}, 600 \mathrm{MHz}\right): \delta 3.20$ (s, $\left.3 \mathrm{H}\right), 3.53$ (t, 2H, J=5.6 Hz), 3.64 (br., $\left.1 \mathrm{H}\right), 3.73$ (s, $3 \mathrm{H}$ ), 3.79 (s, $3 \mathrm{H}), 4.37$ (t, 2H, J=5.6 Hz), 6.48 (d, 2H, J=9.1 Hz), 6.75 (d, 2H, J=8.6 Hz), $7.38-7.45$ $(\mathrm{m}, 3 \mathrm{H}), 7.55(\mathrm{t}, 1 \mathrm{H}, J=7.6 \mathrm{~Hz}), 7.70(\mathrm{dd}, 2 \mathrm{H}, J=7.1,1.0 \mathrm{~Hz}) \mathrm{ppm} .{ }^{13} \mathrm{C}\{1 \mathrm{H}\} \mathrm{NMR}\left(\mathrm{CDCl}_{3}, 150\right.$ $\mathrm{MHz}): \delta 45.9,48.6,51.8,51.9,55.9,114.2$ (2C), 114.4, 115.2 (2C), 124.0, 128.5 (2C), 129.1 (2C), 131.1, 131.8, 133.1, 138.7, 141.0, 152.6, 163.1, 164.4, 187.9 ppm. MS (ESI): m/z =437 [M+H] . HRMS (TOF ES ${ }^{+}$): $\mathrm{m} / \mathrm{z}[\mathrm{M}+\mathrm{H}]^{+}$calculated for $\mathrm{C}_{24} \mathrm{H}_{24} \mathrm{~N}_{2} \mathrm{O}_{6}$ : 437.1707; found: 437.1703. m.p. 136$137^{\circ} \mathrm{C}$. IR (KBr plate): 1061, 1217, 1267, 1399, 1448, 1513, 1638, 1707, 1738, 2954, $3372 \mathrm{~cm}^{-1}$. 
Dimethyl 2-benzoyl-1-\{2-[(4-chlorophenyl)amino]ethyl\}-1H-pyrrole-3,4-dicarboxylate (4c)

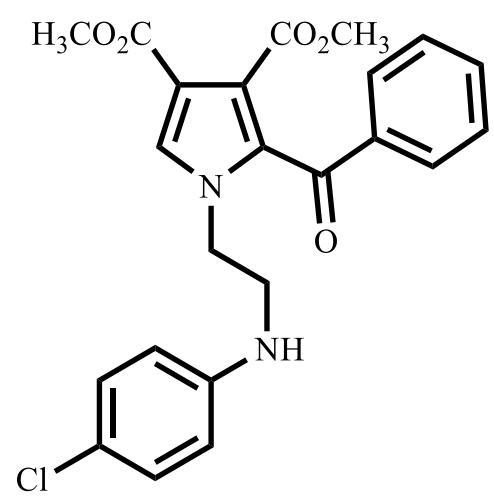

Following GP4 with methyl (2E)-3-[3-(4-chlorophenyl)-2-(3methoxy-3-oxoprop-1-yn-1-yl)-2-phenylimidazolidin-1-yl]prop2-enoate 3c $(0.5 \mathrm{mmol})$ after purification by $\mathrm{SiO}_{2}$ column chromatography $\left(\mathrm{CHCl}_{3}:\right.$ Hexane, $\left.1: 1\right)$ yielded $4 \mathbf{c}(162 \mathrm{mg}, 70 \%)$ as yellow solid.

Following GP5 with 1-(4-chlorophenyl)-2-phenyl-4,5-dihydro$1 H$-imidazole 1c (1.1 mmol) yielded $4 \mathbf{c}(305 \mathrm{mg}, 63 \%)$.

${ }^{1} \mathrm{H} \mathrm{NMR}\left(\mathrm{CDCl}_{3}, 600 \mathrm{MHz}\right): \delta 3.20$ (s, $3 \mathrm{H}$ ), 3.56 (t, 2H, J=5.6 Hz), 3.79 (s, $3 \mathrm{H}$ ), 4.10 (br., $1 \mathrm{H}$ ), 4.37 (t, 2H, J=5.6 Hz), 6.43 (d, 2H, J=9.1 Hz), 7.08 (d, 2H, J=9.1 Hz), 7.41 (s, $1 \mathrm{H}), 7.43$ (t, 2H, $J=8.1 \mathrm{~Hz}), 7.53-7.59(\mathrm{~m}, 1 \mathrm{H}), 7.70(\mathrm{dd}, 2 \mathrm{H}, J=8.3,1.3 \mathrm{~Hz}) \mathrm{ppm} .{ }^{13} \mathrm{C}\{1 \mathrm{H}\} \mathrm{NMR}\left(\mathrm{CDCl}_{3}, 150\right.$ MHz): $\delta$ 45.2, 48.3, 51.8, 52.0, 113.9 (2C), 114.6, 122.7, 124.1, 128.6 (2C), 129.1 (2C), 129.4 (2C), 131.1, 131.7, 133.2, 138.5, 145.6, 163.0, 164.4, 187.9 ppm. MS (ESI): m/z = $463[\mathrm{M}+\mathrm{H}]^{+}$. HRMS (TOF ES ${ }^{+}$): m/z [M+H] ${ }^{+}$calculated for $\mathrm{C}_{23} \mathrm{H}_{21} \mathrm{ClN}_{2} \mathrm{O}_{5}$ : 441.1212; found: 441.1232. m.p. 144-145 ${ }^{\circ} \mathrm{C}$. IR (KBr plate): 733, 1218, 1265, 1399, 1515, 1601, 1632, 1713, 1734, 2947, 3060, $3376 \mathrm{~cm}^{-1}$.

\section{Dimethyl 2-benzoyl-1-(2-\{[4-(trifluoromethyl)phenyl]amino\}ethyl)-1H-pyrrole-3,4- dicarboxylate (4d)}

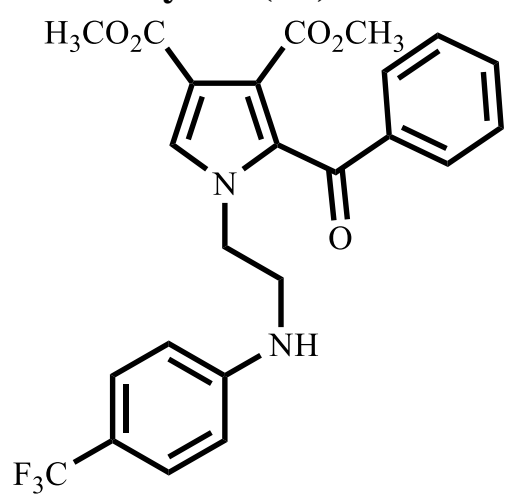

Following GP4 with methyl (2E)-3-\{2-(3-methoxy-3-oxoprop-1yn-1-yl)-2-phenyl-3-[4-(trifluoromethyl)phenyl]imidazolidin-1yl \} prop-2-enoate $3 \mathbf{d}(0.5 \mathrm{mmol})$ after purification by $\mathrm{SiO}_{2}$ column chromatography $\left(\mathrm{CHCl}_{3}:\right.$ Hexane, 1:1) yielded 4d (126mg, 53\%) as yellow solid.

Following GP5 with 2-phenyl-1-[4-(trifluoromethyl)phenyl]-4,5dihydro-1 $H$-imidazole 1d (1.1 mmol) yielded 4d (208mg, 40\%).

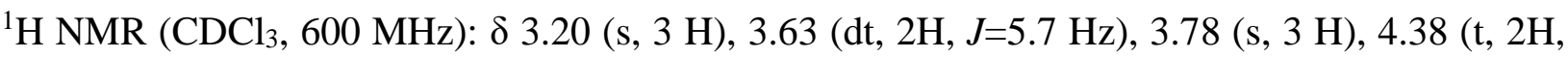
$J=5.8 \mathrm{~Hz}), 4.63$ (t, 1H, J=5.8 Hz), 6.52 (d, 2H, J=8.6 Hz), 7.36 (d, 2H, J=8.6 Hz), 7.42 (t, 2H, $J=8.1 \mathrm{~Hz}), 7.43(\mathrm{~s}, 1 \mathrm{H}), 7.56(\mathrm{t}, 1 \mathrm{H}, J=7.3 \mathrm{~Hz}), 7.68(\mathrm{dd}, 2 \mathrm{H}, J=7.6,1.0 \mathrm{~Hz}) \mathrm{ppm} .{ }^{13} \mathrm{C}\{1 \mathrm{H}\} \mathrm{NMR}$ $\left(\mathrm{CDCl}_{3}, 150 \mathrm{MHz}\right): \delta 44.7,48.2,51.8,51.9,111.9$ (2 C), 114.7, 119.4 (q, $\left.J_{C F}=33.2 \mathrm{~Hz}\right), 124.2$, 124.9 (q, $\left.J_{C F}=270.2 \mathrm{~Hz}\right), 126.8$ (q, $\left.J_{C F}=4.3 \mathrm{~Hz}, 2 \mathrm{C}\right), 128.6$ (2 C), 129.1 (2 C), 131.1, 131.6, 133.3, 138.4, 149.7, 163.0, 164.3, 188.0 ppm. MS (ESI): m/z = $497[\mathrm{M}+\mathrm{Na}]^{+}$. HRMS $\left(\mathrm{TOF} \mathrm{ES}^{+}\right): \mathrm{m} / \mathrm{z}$ $[\mathrm{M}+\mathrm{H}]^{+}$calculated for $\mathrm{C}_{24} \mathrm{H}_{21} \mathrm{~F}_{3} \mathrm{~N}_{2} \mathrm{O}_{5}$ : 475.1475; found: 475.1475. m.p. $113-114^{\circ} \mathrm{C}$. IR $(\mathrm{KBr}$ plate): $838,1066,1323,1535,1615,1715,1742,2952,3410 \mathrm{~cm}^{-1}$. 
Dimethyl 2-benzoyl-1-\{2-[(2-methylphenyl)amino]ethyl $\}-1 H$-pyrrole-3,4-dicarboxylate (4e)

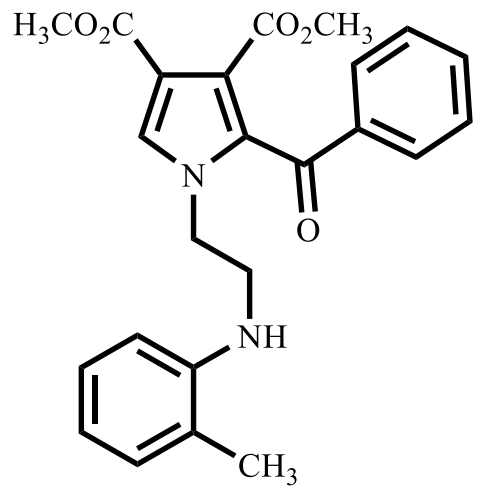

Following GP4 with methyl (2E)-3-[2-(3-methoxy-3-oxoprop-1yn-1-yl)-3-(2-methylphenyl)-2-phenylimidazolidin-1-yl]prop-2enoate $3 \mathbf{e}(0.5 \mathrm{mmol})$ after purification by $\mathrm{SiO}_{2}$ column chromatography $\left(\mathrm{CHCl}_{3}:\right.$ Hexane, 1:1) yielded $4 \mathrm{e}(141 \mathrm{mg}, 67 \%)$ as yellow solid.

Following GP5 with 1-(2-methylphenyl)-2-phenyl-4,5-dihydro$1 H$-imidazole $1 \mathbf{e}(1.1 \mathrm{mmol})$ yielded $4 \mathbf{d}(236 \mathrm{mg}, 51 \%)$.

${ }^{1} \mathrm{H} \mathrm{NMR}\left(\mathrm{CDCl}_{3}, 600 \mathrm{MHz}\right): \delta 2.03(\mathrm{~s}, 3 \mathrm{H}), 3.21(\mathrm{~s}, 3 \mathrm{H}), 3.67(\mathrm{t}, 2 \mathrm{H}, J=5.3 \mathrm{~Hz}), 3.79(\mathrm{~s}, 3 \mathrm{H})$, 3.97 (br., $1 \mathrm{H}), 4.43$ (t, 2H, J=5.6 Hz), 6.58 (d, 1H, J=8.1 Hz), 6.69 (td, 1H, J=7.3, $1.0 \mathrm{~Hz}$ ), 7.05 $(\mathrm{d}, 1 \mathrm{H}, J=6.6 \mathrm{~Hz}), 7.10-7.16(\mathrm{~m}, 1 \mathrm{H}), 7.38(\mathrm{~s}, 1 \mathrm{H}), 7.43(\mathrm{t}, 2 \mathrm{H}, J=7.8 \mathrm{~Hz}), 7.54-7.59(\mathrm{~m}, 1 \mathrm{H})$, $7.72(\mathrm{dd}, 2 \mathrm{H}, J=8.3,1.3 \mathrm{~Hz}) \mathrm{ppm} .{ }^{13} \mathrm{C}\{1 \mathrm{H}\} \mathrm{NMR}\left(\mathrm{CDCl}_{3}, 150 \mathrm{MHz}\right): \delta 17.5,44.9,48.2,51.8$, 51.9, 109.4, 114.5, 117.7, 122.5, 124.2, 127.3, 128.5, 129.1 (2C), 130.6 (2C), 131.0, 131.8, 133.1, 138.7, 144.9, 163.0, 164.4, 188.0 ppm. MS (ESI): m/z = $421[\mathrm{M}+\mathrm{H}]^{+}$. HRMS $\left(\mathrm{TOF} \mathrm{ES}^{+}\right): \mathrm{m} / \mathrm{z}$ $[\mathrm{M}+\mathrm{H}]^{+}$calculated for $\mathrm{C}_{24} \mathrm{H}_{24} \mathrm{~N}_{2} \mathrm{O}_{5}$ : 421.1758; found: 421.1769 . m.p. $142-143^{\circ} \mathrm{C}$. IR (KBr plate): $734,907,1206,1269,1519,1640,1721,1732,2946,3412 \mathrm{~cm}^{-1}$.

\section{1,1'-(2-Benzoyl-1-\{2-[phenylamino]ethyl\}-1H-pyrrole-3,4-diyl)diethanone (4f)}

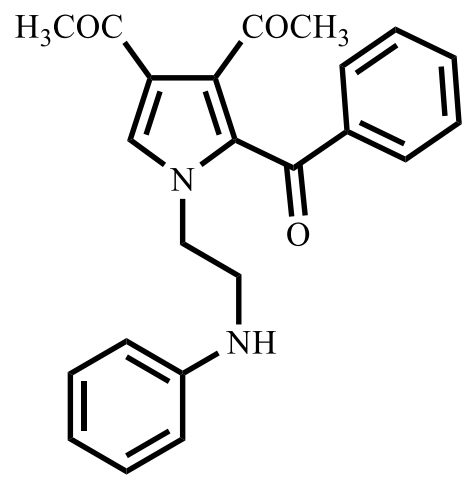

Following GP4 with (3E)-4-[3-phenyl-2-(3-oxobut-1-yn-1-yl)-2phenylimidazolidin-1-yl]but-3-en-2-one $\quad 3 \mathrm{~m} \quad(0.5 \quad \mathrm{mmol})$ after purification by $\mathrm{SiO}_{2}$ column chromatography $\left(\mathrm{CHCl}_{3}: \mathrm{Hexane}, 3: 1\right)$ yielded $4 \mathbf{f}(116 \mathrm{mg}, 60 \%)$ as brown oil.

Following GP5 with 1,2-diphenyl-4,5-dihydro-1H-imidazole 1a (1.1 mmol) yielded $4 \mathbf{f}(168 \mathrm{mg}, 41 \%)$.

${ }^{1} \mathrm{H} \mathrm{NMR}\left(\mathrm{CDCl}_{3}, 600 \mathrm{MHz}\right): \delta 2.21(\mathrm{~s}, 3 \mathrm{H}), 2.37(\mathrm{~s}, 3 \mathrm{H}), 3.60(\mathrm{t}$, 2H, J=5.6 Hz), 4.00 (br., $1 \mathrm{H}), 4.33$ (t, 2H, J=5.6 Hz), 6.52 (dd, 2H, J=8.6, $1.0 \mathrm{~Hz}$ ), 6.71 - 6.76 (m, $1 \mathrm{H}), 7.15$ - 7.19 (m, 2H), 7.27 (s, $1 \mathrm{H}), 7.40$ - 7.42 (m, $2 \mathrm{H}), 7.55$ - 7.58 (m, $1 \mathrm{H}), 7.65$ - 7.69 $(\mathrm{m}, 2 \mathrm{H}) \mathrm{ppm} .{ }^{13} \mathrm{C}\{1 \mathrm{H}\} \mathrm{NMR}\left(\mathrm{CDCl}_{3}, 150 \mathrm{MHz}\right): \delta 27.8,31.6,44.8,48.3,112.9$ (2C), 118.3, 123.2, 128.6 (2C), 129.4 (2C), 129.6 (2C), 131.0, 131.6, 132.8, 133.5, 138.6, 146.9, 188.7, 192.2, 199.6 ppm. HRMS (TOF $\left.\mathrm{ES}^{+}\right)$: $\mathrm{m} / \mathrm{z}[\mathrm{M}+\mathrm{H}]^{+}$calculated for $\mathrm{C}_{23} \mathrm{H}_{22} \mathrm{~N}_{2} \mathrm{O}_{3}$ : 375.1703; found: 375.1710. $\mathrm{R}_{\mathrm{f}}\left(\mathrm{CHCl}_{3}: \mathrm{MeOH}, 100: 1\right)=0.20$. IR (KBr plate): 703, 831, 908, 1191, 1256, 1386, 1512, $1658,1683,3407 \mathrm{~cm}^{-1}$. 
1,1'-(2-Benzoyl-1-\{2-[(4-chlorophenyl)amino]ethyl\}-1H-pyrrole-3,4-diyl)diethanone (4g)<smiles>CC(=O)c1cn(CCNc2ccc(Cl)cc2)c(C(=O)c2ccccc2)c1C(C)=O</smiles>
Following GP4 with (3E)-4-[3-(4-chlorophenyl)-2-(3-oxobut-1yn-1-yl)-2-phenylimidazolidin-1-yl]but-3-en-2-one 3n (0.5 mmol) after purification by $\mathrm{SiO}_{2}$ column chromatography $\left(\mathrm{CHCl}_{3}:\right.$ Hexane, 3:1) yielded $\mathbf{4 g}(140 \mathrm{mg}, 69 \%)$ as yellow oil.

Following GP5 with 1-(4-chlorophenyl)-2-phenyl-4,5-dihydro$1 H$-imidazole $1 \mathbf{c}(1.1 \mathrm{mmol})$ yielded $4 \mathrm{~g}(215 \mathrm{mg}, 48 \%)$.

${ }^{1} \mathrm{H} \mathrm{NMR}\left(\mathrm{CDCl}_{3}, 600 \mathrm{MHz}\right): \delta 2.18(\mathrm{~s}, 3 \mathrm{H}), 2.35(\mathrm{~s}, 3 \mathrm{H}), 3.53(\mathrm{t}$, $2 \mathrm{H}, J=5.6 \mathrm{~Hz}), 4.12$ (br., $1 \mathrm{H}), 4.28$ (t, 2H, $J=5.6 \mathrm{~Hz}), 6.41$ (d, 2H, $J=9.1 \mathrm{~Hz}), 7.07$ (d, 2H, $J=8.6$ Hz), 7.24 (s, $1 \mathrm{H}), 7.39$ (t, 2H, J=7.8 Hz), 7.52 - 7.57 (m, $1 \mathrm{H}), 7.64$ (dd, 2H, J=8.1, 1.5 Hz) ppm. ${ }^{13} \mathrm{C}\{1 \mathrm{H}\} \mathrm{NMR}\left(\mathrm{CDCl}_{3}, 150 \mathrm{MHz}\right): \delta 27.8,31.6,45.0,48.1,113.9(2 \mathrm{C}), 122.8,123.3,128.6(2 \mathrm{C})$, 129.4 (2C), 129.4 (2C), 131.1, 131.3, 132.7, 133.6, 138.5, 145.6, 188.7, 192.2, 199.5 ppm. HRMS (TOF $\mathrm{ES}^{+}$): $\mathrm{m} / \mathrm{z}[\mathrm{M}+\mathrm{H}]^{+}$calculated for $\mathrm{C}_{23} \mathrm{H}_{21} \mathrm{ClN}_{2} \mathrm{O}_{3}$ : 409.1313; found: 409.1319. $\mathrm{R}_{\mathrm{f}}$ $\left(\mathrm{CHCl}_{3}: \mathrm{MeOH}, 100: 1\right)=0.25$. IR (KBr plate): 748, 1252, 1384, 1493, 1598, 1650, 1667, 3011, $3370 \mathrm{~cm}^{-1}$ 


\subsection{Dimethyl 2-benzoyl-1-[2-(arylamino)ethyl]-1H-pyrrole-3,4-dicarboxylates}

Dimethyl 1-\{2-[acetyl(methyl)amino]ethyl\}-2-benzoyl-1H-pyrrole-3,4-dicarboxylate (6a)

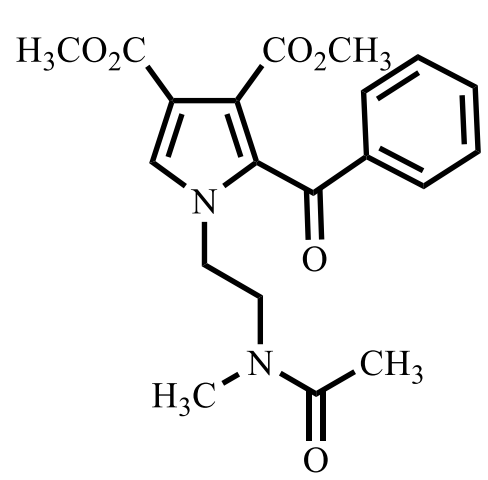

Following GP6 with methyl (2E)-3-[2-(3-methoxy-3-oxoprop-1yn-1-yl)-3-methyl-2-phenylimidazolidin-1-yl]prop-2-enoate $\mathbf{3 f}$ (1 mmol) and acetic anhydride $(1.1 \mathrm{mmol})$ after purification by $\mathrm{SiO}_{2}$ column chromatography $\left(\mathrm{CHCl}_{3}:\right.$ Hexane, $\left.3: 1\right)$ yielded $\mathbf{6 a}(301 \mathrm{mg}$, $78 \%$ ) as yellow oil.

Following GP7 with 1-methyl-2-phenyl-4,5-dihydro- $1 H$-imidazole 1f $(1.1 \mathrm{mmol})$ yielded 6a $(263 \mathrm{mg}, 62 \%)$.

${ }^{1} \mathrm{H} \mathrm{NMR}\left(\mathrm{CDCl}_{3}, 600 \mathrm{MHz}\right.$, a mixture of rotamers in a ratio 0.8: 0.2): $\delta 1.86(\mathrm{~s}, 0.8 \mathrm{H}), 2.02(\mathrm{~s}$, $3 \mathrm{H}), 2.84(\mathrm{~s}, 3 \mathrm{H}), 2.92(\mathrm{~s}, 0.8 \mathrm{H}), 3.19(\mathrm{~s}, 3 \mathrm{H}), 3.71(\mathrm{t}, 2 \mathrm{H}, J=6.3 \mathrm{~Hz}), 3.78(\mathrm{~s}, 3 \mathrm{H}), 4.30(\mathrm{t}, 0.5 \mathrm{H}$, $J=6.1 \mathrm{~Hz}), 4.35$ (t, 2H, J=6.3 Hz), $7.41(\mathrm{~s}, 1 \mathrm{H}), 7.43$ (d, 2H, J=7.6 Hz), 7.55 (t, 1H, J=7.3 Hz), $7.69(\mathrm{~d}, 0.5 \mathrm{H}, J=7.6 \mathrm{~Hz}), 7.71(\mathrm{dd}, 2 \mathrm{H}, J=8.3,1.3 \mathrm{~Hz}) \mathrm{ppm} .{ }^{13} \mathrm{C}\{1 \mathrm{H}\} \mathrm{NMR}\left(\mathrm{CDCl}_{3}, 150 \mathrm{MHz}\right)$ : major rotamer $\delta$ 21.9, 37.6, 47.0, 49.4, 51.8, 51.9, 114.4, 124.5, 128.5 (2C), 129.0 (2C), 130.6, 132.0, 133.1, 138.6, 162.9, 164.3, 171.2, 187.8 ppm; minor rotamer $\delta$ 20.8, 33.7, 47.5, 51.7, 52.0, 114.9, 124.8, 128.6 (2C), 129.0 (2C), 130.5, 131.5, 133.2, 138.4, 162.6, 164.1, 170.9, 187.7 ppm. MS (ESI): $\mathrm{m} / \mathrm{z}=409[\mathrm{M}+\mathrm{Na}]^{+}$. HRMS $\left(\right.$TOF ES $\left.{ }^{+}\right): \mathrm{m} / \mathrm{z}[\mathrm{M}+\mathrm{H}]^{+}$calculated for $\mathrm{C}_{20} \mathrm{H}_{22} \mathrm{~N}_{2} \mathrm{O}_{6}$ : 387.1551; found: 387.1554. $\mathrm{R}_{\mathrm{f}}\left(\mathrm{CHCl}_{3}: \mathrm{MeOH}, 100: 1\right)=0.28$. IR (KBr plate): 908, 1063, 1213, $1269,1399,1643,1722,2951 \mathrm{~cm}^{-1}$.

\section{Dimethyl 2-benzoyl-1-\{2-[(tert-butoxycarbonyl)(methyl)amino]ethyl\}-1H-pyrrole-3,4- dicarboxylate (6b)}

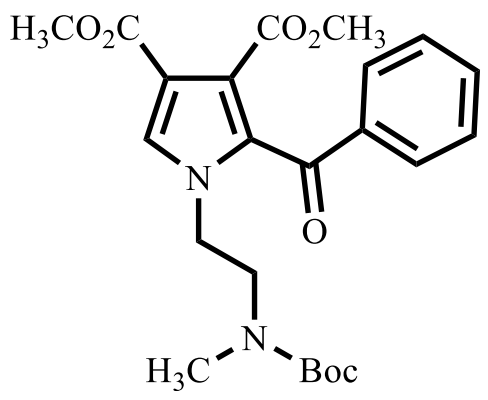

Following GP6 with methyl (2E)-3-[2-(3-methoxy-3-oxoprop-1yn-1-yl)-3-methyl-2-phenylimidazolidin-1-yl]prop-2-enoate $\mathbf{3 f}$ (1 $\mathrm{mmol})$ and di-tert-butyl dicarbonate $(1.1 \mathrm{mmol})$ after purification by $\mathrm{SiO}_{2}$ column chromatography $\left(\mathrm{CHCl}_{3}\right.$ :Hexane, 3:1) yielded $\mathbf{6 b}$ (214mg, 48\%) as yellow oil.

Following GP7 with 1-methyl-2-phenyl-4,5-dihydro- $1 H$ -

imidazole 1f (1.1 mmol) yielded $6 \mathbf{b}(196 \mathrm{mg}, 40 \%)$.

${ }^{1} \mathrm{H} \mathrm{NMR}\left(\mathrm{CDCl}_{3}, 600 \mathrm{MHz}\right.$, a mixture of rotamers in a ratio 1: 1): $\delta 1.36$ (br., $4.5 \mathrm{H}$ ), 1.42 (br., 4.5 H), 2.69 (br., 1.5 H), 2.76 (br., 1.5 H), 3.18 (s, 3 H), 3.53 - 3.60 (m, 1 H), 3.60 - 3.67 (m, 1 H), 3.77 (s, 3 H), 4.24 - 4.32 (m, 1 H), 4.34 - 4.40 (m, 1 H), 7.34 (br., 0.5 H), 7.39 (br., 0.5 H), $7.40-7.44(\mathrm{~m}, 2 \mathrm{H}), 7.52-7.56(\mathrm{~m}, 1 \mathrm{H}), 7.66-7.76(\mathrm{~m}, 1 \mathrm{H}) \mathrm{ppm} .{ }^{13} \mathrm{C}\{1 \mathrm{H}\} \mathrm{NMR}\left(\mathrm{CDCl}_{3}, 150\right.$ MHz): $\delta 28.3$ (1.5 C), 28.4 (1.5 C), 34.8, 35.9, 47.2, 47.6, 49.9, 50.2, 51.6, 51.7, 51.8 (1 C), 80.1, 80.2, 114.2, 114.5, 124.4, 124.5, 128.4 (2 C), 128.9 (1 C), 129.0 (1 C), 130.6, 130.8, 131.6, 132.2, 
132.9 (1 C), 138.7, 155.3, 155.7, 162.9 (1 C), 164.3, 164.4, 187.6, 187.6, 187.6, 187.7 ppm. MS (ESI): $\mathrm{m} / \mathrm{z}=467[\mathrm{M}+\mathrm{Na}]^{+}$. HRMS $\left(\mathrm{TOF} \mathrm{ES}^{+}\right): \mathrm{m} / \mathrm{z}[\mathrm{M}+\mathrm{H}]^{+}$calculated for $\mathrm{C}_{23} \mathrm{H}_{28} \mathrm{~N}_{2} \mathrm{O}_{7}$ : 446.2047; found: 446.2052. $\mathrm{R}_{\mathrm{f}}$ (Ethyl acetate:Hexane,1:1)=0.3. IR (KBr plate): 697, 767, 906, 1063, 1155, $1214,1268,1395,1644,1696,1722,2951 \mathrm{~cm}^{-1}$.

Dimethyl 2-benzoyl-1-(2-\{[(3-chlorophenyl)carbamoyl](methyl)amino\}ethyl)-1H-pyrrole3,4-dicarboxylate (6c)<smiles>CC(=O)c1cn(CCN(C)C(=O)Nc2cccc(Cl)c2)c(C(=O)c2ccccc2)c1C(C)=O</smiles>

Following GP6 with methyl (2E)-3-[2-(3-methoxy-3oxoprop-1-yn-1-yl)-3-methyl-2-phenylimidazolidin-1-yl] prop-2-enoate 3f $(1 \mathrm{mmol})$ and 3-chlorophenyl isocyanate (1.1 mmol) after purification by $\mathrm{SiO}_{2}$ column chromatography $\left(\mathrm{CHCl}_{3}: H e x a n e, 3: 1\right)$ yielded 6c (303mg, $61 \%$ ) as yellow oil.

Following GP7 with 1-methyl-2-phenyl-4,5-dihydro-1 $H$ imidazole $\mathbf{1 f}(1.1 \mathrm{mmol})$ yielded $\mathbf{6 c}(245 \mathrm{mg}, 45 \%)$.

${ }^{1} \mathrm{H} \mathrm{NMR}\left(\mathrm{CDCl}_{3}, 600 \mathrm{MHz}\right): \delta 3.01$ (s, $\left.3 \mathrm{H}\right), 3.22$ (s, $\left.3 \mathrm{H}\right), 3.78$ - 3.81 (m, $\left.2 \mathrm{H}\right), 3.79$ (s, 3H), 4.33 (t, 2H, J=7.3 Hz), $6.98(\mathrm{dd}, 1 \mathrm{H}, J=8.1,1.5 \mathrm{~Hz}), 7.18(\mathrm{t}, 1 \mathrm{H}, J=8.1 \mathrm{~Hz}), 7.38-7.44(\mathrm{~m}, 2 \mathrm{H}), 7.46$ (t, 2H, J=7.6 Hz), 7.49 (s, $1 \mathrm{H}), 7.59$ (t, 1H, J=7.1 Hz), $7.62(\mathrm{~s}, 1 \mathrm{H}), 7.76$ (d, 2H, J=8.1 Hz) ppm. ${ }^{13} \mathrm{C}\{1 \mathrm{H}\} \mathrm{NMR}\left(\mathrm{CDCl}_{3}, 150 \mathrm{MHz}\right): \delta 35.6,47.5,50.6,51.9,52.1,115.0,117.7,119.9,122.9$, 125.2, 128.7 (2C), 129.3 (2C), 129.8, 130.4, 132.1, 133.5, 134.4, 138.4, 140.7, 155.0, 162.7, 164.2, 188.4 ppm. MS (ESI): $\mathrm{m} / \mathrm{z}=520[\mathrm{M}+\mathrm{Na}]^{+}$. HRMS $\left(\mathrm{TOF} \mathrm{ES}^{+}\right): \mathrm{m} / \mathrm{z}[\mathrm{M}-\mathrm{H}]^{+}$calculated for $\mathrm{C}_{25} \mathrm{H}_{24} \mathrm{ClN}_{3} \mathrm{O}_{6}$ : 496.1281; found: 496.1287. $\mathrm{R}_{\mathrm{f}}\left(\mathrm{CHCl}_{3}: \mathrm{MeOH}, 100: 1\right)=0.3$. IR (KBr plate): 767, $1214,1268,1532,1649,1657,1723,2951,3378 \mathrm{~cm}^{-1}$.

\section{Dimethyl 2-benzoyl-1-(2-\{benzyl[(3-chlorophenyl)carbamoyl]amino\}ethyl)-1H-pyrrole-3,4-} dicarboxylate (6d)<smiles>CC(=O)c1cn(CCN(Cc2ccccc2)C(=O)Nc2cccc(Cl)c2)c(C(=O)c2ccccc2)c1C(C)=O</smiles>

Following GP6 with methyl (2E)-3-[3-benzyl-2-(3-methoxy-3oxoprop-1-yn-1-yl)-2-phenylimidazolidin-1-yl]prop-2-enoate $\mathbf{3 g}$ (1 $\mathrm{mmol})$ and 3-chlorophenyl isocyanate $(1.1 \mathrm{mmol})$ after purification by $\mathrm{SiO}_{2}$ column chromatography $\left(\mathrm{CHCl}_{3}: \mathrm{Hexane}\right.$, $3: 1)$ yielded $\mathbf{6 d}(372 \mathrm{mg}, 65 \%)$ as colorless oil.

Following GP7 with 1-benzyl-2-phenyl-4,5-dihydro-1 $H$ imidazole $1 \mathrm{~g}$ (2.25 mmol) yielded 6d (760mg, 59\%).

${ }^{1} \mathrm{H}$ NMR $\left(\mathrm{CDCl}_{3}, 600 \mathrm{MHz}\right): \delta 3.21(\mathrm{~s}, 3 \mathrm{H}), 3.80(\mathrm{~s}, 3 \mathrm{H}), 3.83$ (t, 2H, J=8.1 Hz), 4.09 (t, 2H, J=7.3 Hz), 4.66 (s, 2 H), 6.99 (dd, 1H, J=8.1, $1.5 \mathrm{~Hz}), 7.19$ (t, 1H, 
$J=8.1 \mathrm{~Hz}), 7.26(\mathrm{~s}, 1 \mathrm{H}), 7.31-7.37(\mathrm{~m}, 3 \mathrm{H}), 7.37$ - $7.42(\mathrm{~m}, 2 \mathrm{H}), 7.46(\mathrm{t}, 2 \mathrm{H}, J=7.6 \mathrm{~Hz}), 7.55$ $(\mathrm{d}, 1 \mathrm{H}, J=7.1 \mathrm{~Hz}), 7.60(\mathrm{t}, 1 \mathrm{H}, J=7.1 \mathrm{~Hz}), 7.75(\mathrm{~d}, 3 \mathrm{H}, J=8.6 \mathrm{~Hz}), 7.95$ (br., $1 \mathrm{H}) \mathrm{ppm} .{ }^{13} \mathrm{C}\{1 \mathrm{H}\}$ $\mathrm{NMR}\left(\mathrm{CDCl}_{3}, 150 \mathrm{MHz}\right): \delta$ 47.8, 48.9, 51.6, 52.0, 52.1, 115.2, 117.8, 120.0, 122.9, 125.4, 128.0 (2C), 128.2, 128.7 (2C), 129.2 (2C), 129.3 (2C), 129.9, 130.1, 132.2, 133.6, 134.5, 137.7, 138.3, 140.9, 155.1, 162.7, 164.2, 188.6 ppm. MS (ESI): m/z = $574[\mathrm{M}+\mathrm{H}]^{+}$. HRMS (TOF ES $\left.{ }^{+}\right): \mathrm{m} / \mathrm{z}$ [M$\mathrm{H}]^{+}$calculated for $\mathrm{C}_{31} \mathrm{H}_{28} \mathrm{ClN}_{3} \mathrm{O}_{6}$ : 572.1594; found: 572.1603. $\mathrm{R}_{\mathrm{f}}\left(\mathrm{CHCl}_{3}: \mathrm{MeOH}, 400: 1\right)=0.33$. IR (KBr plate): 698, 1224, 1483, 1536, 1593, 1628, 1667, 1706, 1736, 3125, $3367 \mathrm{~cm}^{-1}$.

\section{Dimethyl 2-benzoyl-1-(2-\{methyl[(4-methylphenyl)carbamoyl]amino\}ethyl)-1H-pyrrole-}

\section{3,4-dicarboxylate (6e)}

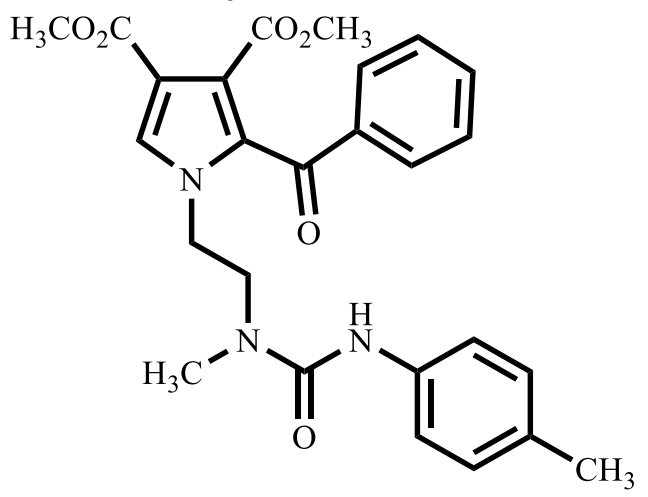

Following GP6 with methyl (2E)-3-[2-(3-methoxy-3oxoprop-1-yn-1-yl)-3-methyl-2-phenylimidazolidin-1yl]prop-2-enoate $3 \mathbf{f}(1 \mathrm{mmol})$ and p-tolyl isocyanate (1.1 mmol) after purification by $\mathrm{SiO}_{2}$ column chromatography $\left(\mathrm{CHCl}_{3}:\right.$ Hexane, $\left.3: 1\right)$ yielded $\mathbf{6 e}(310 \mathrm{mg}, 65 \%)$ as yellow oil.

Following GP7 with 1-methyl-2-phenyl-4,5-dihydro- $1 H$ -

imidazole $\mathbf{1 f}(1.1 \mathrm{mmol})$ yielded $\mathbf{6 e}(290 \mathrm{mg}, 55 \%)$.

${ }^{1} \mathrm{H} \mathrm{NMR}\left(\mathrm{CDCl}_{3}, 600 \mathrm{MHz}\right): \delta 2.28$ (s, 3H), 2.95 (s, $\left.3 \mathrm{H}\right), 3.21$ (s, $\left.3 \mathrm{H}\right), 3.74$ - 3.77 (t, 2H, J=7.1 Hz), 3.78 (s, $3 \mathrm{H}), 4.33$ (t, 2H, J=6.8 Hz), 7.02 (br., $1 \mathrm{H}), 7.07$ (d, 2H, J=8.1 Hz), 7.33 (d, 2H, $J=8.1 \mathrm{~Hz}), 7.43(\mathrm{t}, 2 \mathrm{H}, J=7.8 \mathrm{~Hz}), 7.48(\mathrm{~s}, 1 \mathrm{H}), 7.53-7.59(\mathrm{~m}, 1 \mathrm{H}), 7.73(\mathrm{dd}, 2 \mathrm{H}, J=8.1,1.5 \mathrm{~Hz})$ ppm. ${ }^{13} \mathrm{C}\{1 \mathrm{H}\} \mathrm{NMR}\left(\mathrm{CDCl}_{3}, 150 \mathrm{MHz}\right): \delta 20.8,35.6,47.6,50.6,51.8,52.0,114.8,120.3(2 \mathrm{C})$, 124.9, 128.6 (2C), 129.1 (2C), 129.4 (2C), 130.4, 132.1, 132.7, 133.2, 136.5, 138.5, 155.6, 162.8, 164.3, $188.1 \mathrm{ppm}$. MS (ESI): m/z $=478[\mathrm{M}+\mathrm{H}]^{+}$. HRMS $\left(\mathrm{TOF} \mathrm{ES}^{+}\right): \mathrm{m} / \mathrm{z}[\mathrm{M}-\mathrm{H}]^{+}$calculated for $\mathrm{C}_{26} \mathrm{H}_{27} \mathrm{~N}_{3} \mathrm{O}_{6}: 478.1973$; found: 478.2019. $\mathrm{R}_{\mathrm{f}}\left(\mathrm{CHCl}_{3}: \mathrm{MeOH}, 100: 1\right)=0.4$. IR (KBr plate): 1064, $1215,1267,1509,1655,1719,1735,2947,3118,3390 \mathrm{~cm}^{-1}$.

Dimethyl 2-benzoyl-1-(2-\{benzyl[(4-methylphenyl)carbamoyl]amino\}ethyl)-1H-pyrrole-3,4dicarboxylate (6f)<smiles>CC(=O)c1cn(CCN(Cc2ccccc2)C(=O)Nc2ccc(C)cc2)c(C(=O)c2ccccc2)c1C(=O)O</smiles>
Following GP6 with methyl (2E)-3-[3-benzyl-2-(3methoxy-3-oxoprop-1-yn-1-yl)-2-phenylimidazolidin-1yl]prop-2-enoate $\mathbf{3 g}$ ( $1 \mathrm{mmol})$ and p-tolyl isocyanate $(1.1$ mmol) after purification by $\mathrm{SiO}_{2}$ column chromatography $\left(\mathrm{CHCl}_{3}:\right.$ Hexane, $\left.3: 1\right)$ yielded $\mathbf{6 f}(380 \mathrm{mg}, 69 \%)$ as yellow oil. 
Following GP7 with 1-benzyl-2-phenyl-4,5-dihydro-1H-imidazole $1 \mathrm{~g}(1.1 \mathrm{mmol})$ yielded $\mathbf{6 f}$ (365mg, 60\%).

${ }^{1} \mathrm{H}$ NMR (DMSO- $\left.d_{6}, 600 \mathrm{MHz}\right): \delta 2.23(\mathrm{~s}, 3 \mathrm{H}), 3.14(\mathrm{~s}, 3 \mathrm{H}), 3.66(\mathrm{t}, 2 \mathrm{H}, J=6.1 \mathrm{~Hz}), 3.72(\mathrm{~s}, 3$ H), $4.50(\mathrm{~s}, 2 \mathrm{H}), 4.51(\mathrm{t}, 2 \mathrm{H}, J=6.1 \mathrm{~Hz}), 7.01(\mathrm{~d}, 2 \mathrm{H}, J=8.6 \mathrm{~Hz}), 7.21(\mathrm{~d}, 2 \mathrm{H}, J=2.5 \mathrm{~Hz}), 7.22(\mathrm{~d}$, $2 \mathrm{H}, J=3.5 \mathrm{~Hz}), 7.24(\mathrm{t}, 2 \mathrm{H}, J=7.3 \mathrm{~Hz}), 7.32(\mathrm{t}, 2 \mathrm{H}, J=7.8 \mathrm{~Hz}), 7.85$ (d, 2H, J=8.6 Hz), $8.01(\mathrm{~s}, 1$ H), 8.20 (d, 2H, J=8.1 Hz), 8.27 (s, $1 \mathrm{H})$ ppm. ${ }^{13} \mathrm{C}\{1 \mathrm{H}\}$ NMR (DMSO- $\left.d_{6}, 150 \mathrm{MHz}\right): \delta 20.3,46.5$, 46.8, 49.2, 51.6, 51.8, 113.3, 120.4 (2C), 123.3 (2C), 124.7, 127.0 (2C), 127.1, 128.5 (2C), 128.6 (2C), 129.4, 130.0 (2C), 130.9, 133.6, 137.4, 138.1, 143.0, 149.5, 155.1, 162.2, 163.8, 185.5 ppm. HRMS (TOF ES ${ }^{+}$): m/z [M-H] $]^{+}$calculated for $\mathrm{C}_{32} \mathrm{H}_{31} \mathrm{~N}_{3} \mathrm{O}_{6}: 552.2140$; found: 552.2154. $\mathrm{R}_{\mathrm{f}}$ (Ethyl acetate:Hexane, 1:1)=0.3. IR (KBr plate): 1067, 1218, 1270, 1513, 1659, 1723, 1738, 2951, 3119, $3393 \mathrm{~cm}^{-1}$.

\section{Dimethyl 2-benzoyl-1-\{2-[benzyl(ethylcarbamothioyl)amino]ethyl $\}-1 H$-pyrrole-3,4-} dicarboxylate $(6 \mathrm{~g})$

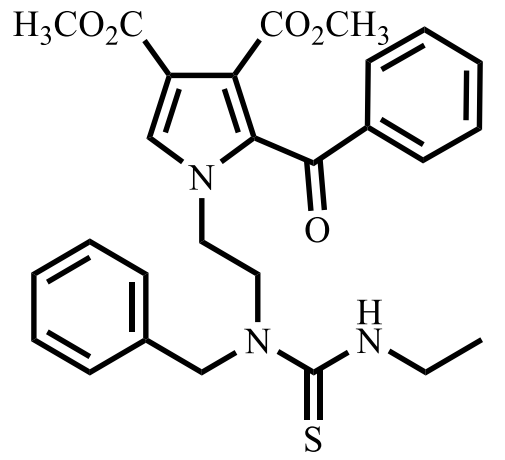

Following GP6 with methyl (2E)-3-[3-benzyl-2-(3-methoxy-3oxoprop-1-yn-1-yl)-2-phenylimidazolidin-1-yl]prop-2-enoate $\quad \mathbf{3 g}$ $(1 \mathrm{mmol})$ and ethyl isothiocyanate $(1.1 \mathrm{mmol})$ after purification by $\mathrm{SiO}_{2}$ column chromatography $\left(\mathrm{CHCl}_{3}: \mathrm{Hexane}, 3: 1\right)$ yielded $\mathbf{6 g}$ (350mg, 69\%) as yellow oil.

Following GP7 with 1-benzyl-2-phenyl-4,5-dihydro-1 $H$-imidazole $1 \mathrm{~g}(1.1 \mathrm{mmol})$ yielded $\mathbf{6 g}(347 \mathrm{mg}, 62 \%)$.

${ }^{1} \mathrm{H} \mathrm{NMR}\left(\mathrm{CDCl}_{3}, 600 \mathrm{MHz}\right): \delta 1.19(\mathrm{t}, 3 \mathrm{H}, J=7.3 \mathrm{~Hz}), 3.21(\mathrm{~s}, 3 \mathrm{H}), 3.67$ - $3.71(\mathrm{~m}, 2 \mathrm{H}), 3.80(\mathrm{~s}$, $3 \mathrm{H}), 4.12$ (m, 2 H), 4.26 (m, 2 H), 4.94 (m, 2 H), 6.45 (br., 1 H), 7.28 - 7.32 (m, 3 H), 7.35 - 7.37 $(\mathrm{m}, 3 \mathrm{H}), 7.43(\mathrm{t}, 2 \mathrm{H}, J=7.6 \mathrm{~Hz}), 7.57(\mathrm{t}, 1 \mathrm{H}, J=7.6 \mathrm{~Hz}), 7.69(\mathrm{~d}, 2 \mathrm{H}, J=7.1 \mathrm{~Hz}) \mathrm{ppm} .{ }^{13} \mathrm{C}\{1 \mathrm{H}\}$ $\operatorname{NMR}\left(\mathrm{CDCl}_{3}, 150 \mathrm{MHz}\right): \delta 14.3,41.7,46.6,51.9,52.0$ (2C), 55.2, 115.0, 125.1, 127.2 (2C), 128.2, $128.6(2 \mathrm{C}), 129.2$ (2C), 129.2 (2C), 130.2, 132.2, 133.3, 136.2, 138.5, 162.8, 164.3, 182.5, 188.1 ppm. MS (ESI): m/z = $508[\mathrm{M}+\mathrm{H}]^{+}$. HRMS $\left(\mathrm{TOF} \mathrm{ES}^{+}\right): \mathrm{m} / \mathrm{z}[\mathrm{M}+\mathrm{H}]^{+}$calculated for $\mathrm{C}_{27} \mathrm{H}_{29} \mathrm{~N}_{3} \mathrm{O}_{5} \mathrm{~S}$ : 508.1901; found: 508.1902. $\mathrm{R}_{\mathrm{f}}$ (Ethyl acetate: Hexane, 1:2) = 0.29. $\mathrm{IR}$ (KBr plate): 698, 732, 1057, $1159,1213,1269,1535,1620,1720,2949,3365 \mathrm{~cm}^{-1}$. 
Dimethyl 2-benzoyl-1-\{2-[methyl(phenylcarbamothioyl)amino]ethyl\}-1H-pyrrole-3,4dicarboxylate (6h)<smiles>COC(=O)c1c(C(C)=O)cn(CCN(C)C(=S)Nc2ccccc2)c1C(=O)c1ccccc1</smiles>

Following GP6 with methyl (2E)-3-[2-(3-methoxy-3-oxoprop1-yn-1-yl)-3-methyl-2-phenylimidazolidin-1-yl]prop-2-enoate 3f $(1 \mathrm{mmol})$ and phenyl isocyanate $(1.1 \mathrm{mmol})$ after purification by $\mathrm{SiO}_{2}$ column chromatography $\left(\mathrm{CHCl}_{3}:\right.$ Hexane, $\left.3: 1\right)$ yielded 6h (311mg, 57\%) as yellow oil.

Following GP7 with 1-methyl-2-phenyl-4,5-dihydro- $1 \mathrm{H}$ imidazole $1 \mathbf{f}(1.1 \mathrm{mmol})$ yielded $\mathbf{6 h}(270 \mathrm{mg}, 51 \%)$.

${ }^{1} \mathrm{H}$ NMR $\left(\mathrm{CDCl}_{3}, 600 \mathrm{MHz}\right): \delta 3.20(\mathrm{~s}, 3 \mathrm{H}), 3.23(\mathrm{~s}, 3 \mathrm{H}), 3.81(\mathrm{~s}, 3 \mathrm{H}), 4.31(\mathrm{t}, 2 \mathrm{H}, J=6.8 \mathrm{~Hz})$, $4.55(\mathrm{t}, 2 \mathrm{H}, J=6.8 \mathrm{~Hz}), 7.20-7.24(\mathrm{~m}, 1 \mathrm{H}), 7.26(\mathrm{~s}, 1 \mathrm{H}), 7.36(\mathrm{~d}, 4 \mathrm{H}, J=4.0 \mathrm{~Hz}), 7.45(\mathrm{t}, 2 \mathrm{H}$, $J=7.6 \mathrm{~Hz}), 7.56(\mathrm{~s}, 1 \mathrm{H}), 7.57-7.60(\mathrm{~m}, 1 \mathrm{H}), 7.71-7.75(\mathrm{~m}, 2 \mathrm{H}) \mathrm{ppm} .{ }^{13} \mathrm{C}\{1 \mathrm{H}\} \mathrm{NMR}\left(\mathrm{CDCl}_{3}\right.$, $150 \mathrm{MHz}): \delta 39.3,46.6,51.9,52.0,55.0,55.0,114.9,126.0(2 \mathrm{C}), 126.3,128.6(2 \mathrm{C}), 128.9(2 \mathrm{C})$, 129.2 (2C), 130.6, 132.3, 133.3, 138.6, 139.5, 162.8, 164.3, 182.5, 188.1 ppm. MS (ESI): m/z = $520[\mathrm{M}+\mathrm{Na}]^{+}$; HRMS (TOF $\mathrm{ES}^{+}$): $\mathrm{m} / \mathrm{z}[\mathrm{M}-\mathrm{H}]^{+}$calculated for $\mathrm{C}_{25} \mathrm{H}_{25} \mathrm{~N}_{3} \mathrm{O}_{5} \mathrm{~S}: 480.1588$; found: 480.1593. $\mathrm{R}_{\mathrm{f}}($ Ethyl acetate:Hexane, 1:1)=0.3. IR (KBr plate): 731, 907, 1213, 1266, 1719, 1735, $2947,3335 \mathrm{~cm}^{-1}$.

Dimethyl 1-(2-\{benzyl[(4-methylphenyl)carbamoyl]amino\}ethyl)-2-(4-methoxybenzoyl)-1Hpyrrole-3,4-dicarboxylate (6i)

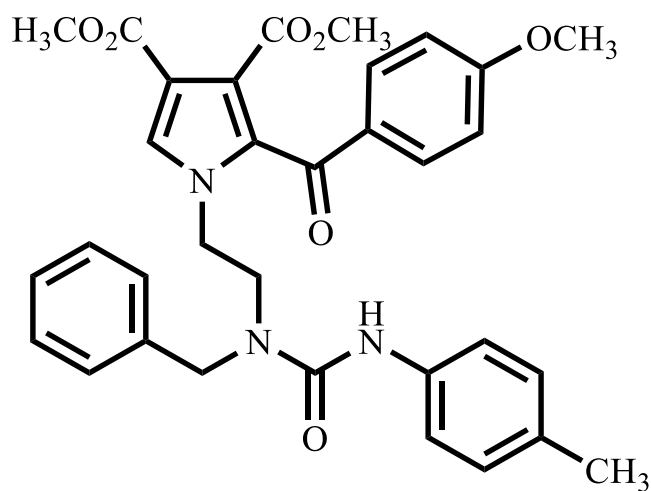

Following GP6 with methyl (2E)-3-[3-benzyl-2-(3methoxy-3-oxoprop-1-yn-1-yl)-2-(4-methoxyphenyl) imidazolidin-1-yl]prop-2-enoate $\mathbf{3 h}(1 \mathrm{mmol})$ and $\mathrm{p}$ tolyl isocyanate $(1.1 \mathrm{mmol})$ after purification by $\mathrm{SiO}_{2}$ column chromatography $\left(\mathrm{CHCl}_{3}:\right.$ Hexane, $\left.3: 1\right)$ yielded $6 \mathbf{i}$ (191mg, 43\%) as yellow oil.

Following GP7 with 1-benzyl-2-(4-methoxyphenyl)4,5-dihydro- $1 H$-imidazole $\mathbf{1 h}(1.1 \mathrm{mmol})$ yielded $\mathbf{6 i}(244 \mathrm{mg}, 38 \%)$.

${ }^{1} \mathrm{H} \mathrm{NMR}\left(\mathrm{CDCl}_{3}, 600 \mathrm{MHz}\right): \delta 2.27$ (s, $\left.3 \mathrm{H}\right), 3.30$ (s, $\left.3 \mathrm{H}\right), 3.76$ - 3.83 (m, $\left.5 \mathrm{H}\right), 3.84(\mathrm{~s}, 3 \mathrm{H}), 4.08$ (m, 2 H), 4.58 (s, 2 H), 6.90 (dd, 2H, J=9.1, 2.52 Hz), 7.07 (d, 2H, J=7.1 Hz), 7.26 (s, $1 \mathrm{H}), 7.27$ 7.33 (m, $3 \mathrm{H}), 7.35$ (t, 2H, J=7.1 Hz), 7.42 (d, 2H, J=7.1 Hz), 7.58 (br., $1 \mathrm{H}), 7.73$ (dd, 2H, J=8.8, $2.8 \mathrm{~Hz}) \mathrm{ppm} .{ }^{13} \mathrm{C}\{1 \mathrm{H}\} \mathrm{NMR}\left(\mathrm{CDCl}_{3}, 150 \mathrm{MHz}\right): \delta 20.8,47.5,49.0,51.5,51.8,52.1,55.6,113.9$ (2C), 114.7, 120.2 (2C), 123.7, 127.7 (2C), 128.0, 129.1 (2C), 129.3 (2C), 130.8, 131.1, 131.5, 131.8 (2C), 132.4, 136.8, 137.8, 155.5, 162.8, 164.1, 164.3, 186.8 ppm. MS (ESI): m/z = 584 $[\mathrm{M}+\mathrm{H}]^{+}$. HRMS (TOF ES ${ }^{+}$): m/z [M-H] $]^{+}$calculated for $\mathrm{C}_{33} \mathrm{H}_{33} \mathrm{~N}_{3} \mathrm{O}_{7}$ : 582.2245; found: 582.2259. 
$\mathrm{R}_{\mathrm{f}}\left(\mathrm{CHCl}_{3}\right)=0.42 . \mathrm{IR}(\mathrm{KBr}$ plate): 1263, 1514, 1529, 1596, 1640, 1658, 1723, 1737, 2948, 3383 $\mathrm{cm}^{-1}$.

\section{Dimethyl 1-(2-\{benzyl[(4-methylphenyl)carbamoyl]amino\}ethyl)-2-(4-nitrobenzoyl)-1H-} pyrrole-3,4-dicarboxylate $(6 \mathbf{j})$

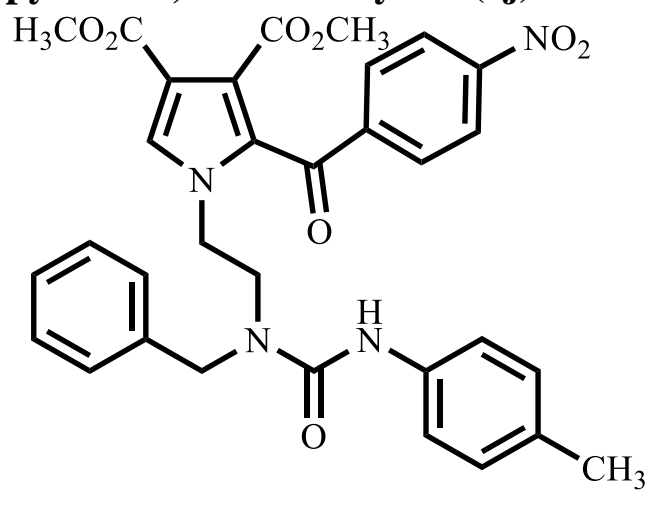

Following GP6 with methyl (2E)-3-[3-benzyl-2-(3methoxy-3-oxoprop-1-yn-1-yl)-2-(4-nitrophenyl) imidazolidin-1-yl]prop-2-enoate $\mathbf{3 j}$ (1 mmol) and p-tolyl isocyanate $(1.1 \mathrm{mmol})$ after purification by $\mathrm{SiO}_{2}$ column chromatography $\left(\mathrm{CHCl}_{3}:\right.$ Hexane, 3:1) yielded $\mathbf{6 j}$ (371mg, 62\%) as yellow solid.

Following GP7 with 1-benzyl-2-(4-nitrophenyl)-4,5dihydro- $1 H$-imidazole $\mathbf{1 j}$ (1.1 mmol) yielded $\mathbf{6 j}$ (362 $\mathrm{mg}, 55 \%)$.

${ }^{1} \mathrm{H} \mathrm{NMR}\left(\mathrm{CDCl}_{3}, 600 \mathrm{MHz}\right): \delta 2.28(\mathrm{~s}, 3 \mathrm{H}), 3.28$ (s, $\left.3 \mathrm{H}\right), 3.80(\mathrm{~s}, 3 \mathrm{H}), 3.83$ (t, 2H, J=7.3 Hz), 4.30 (t, 2H, J=7.1 Hz), 4.57 (s, $2 \mathrm{H}), 7.06$ (d, 2H, J=8.1 Hz), 7.12 (br., $1 \mathrm{H}), 7.26-7.35$ (m, $5 \mathrm{H}$ ), $7.36-7.42(\mathrm{~m}, 3 \mathrm{H}), 7.84(\mathrm{~d}, 2 \mathrm{H}, J=9.1 \mathrm{~Hz}), 8.24(\mathrm{~d}, 2 \mathrm{H}, J=8.6 \mathrm{~Hz}) \mathrm{ppm} .{ }^{13} \mathrm{C}\{1 \mathrm{H}\} \mathrm{NMR}\left(\mathrm{CDCl}_{3}\right.$, $150 \mathrm{MHz}): \delta 20.8,48.1,49.2,51.8,52.0,52.3,115.3,120.2(2 \mathrm{C}), 123.5$ (2C), 126.6, $127.4(2 \mathrm{C})$, 128.2, 128.9, 129.3 (2C), 129.4 (2C), 130.0 (2C), 132.9, 133.2, 136.4, 137.2, 143.5, 150.1, 155.5, 162.4, 164.2, 186.0 ppm. MS (ESI): m/z = $599[\mathrm{M}+\mathrm{H}]^{+}$. HRMS $\left(\mathrm{TOF} \mathrm{ES}^{+}\right): \mathrm{m} / \mathrm{z}[\mathrm{M}-\mathrm{H}]^{+}$calculated for $\mathrm{C}_{32} \mathrm{H}_{30} \mathrm{~N}_{4} \mathrm{O}_{8}$ : 597.1991; found: 597.2009. m.p. $147-148^{\circ} \mathrm{C}$. IR (KBr plate): $1211,1511,1526$, $1592,1638,1655,1720,1734,2944,3381 \mathrm{~cm}^{-1}$.

\section{Dimethyl 1-(2-\{benzyl[(3-chlorophenyl)carbamoyl]amino\}ethyl)-2-(4-chlorobenzoyl)-1H-} pyrrole-3,4-dicarboxylate (6k)

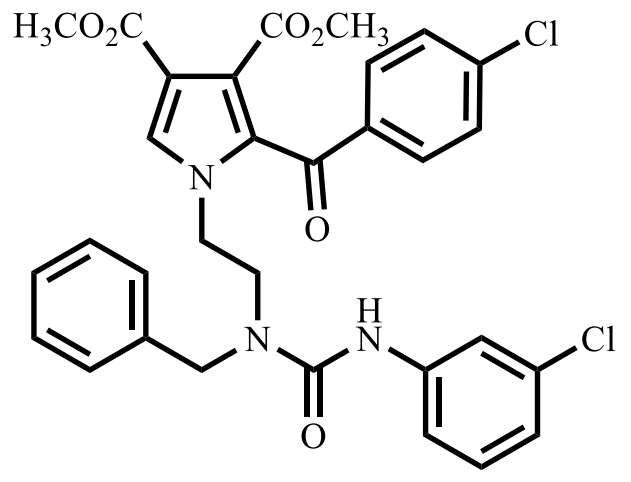

Following GP6 with methyl (2E)-3-[3-benzyl-2-(4chlorophenyl)-2-(3-methoxy-3-oxoprop-1-yn-1-yl) imidazolidin-1-yl]prop-2-enoate $\mathbf{3 i}(1 \mathrm{mmol})$ and 3 chlorophenyl isocyanate $(1.1 \mathrm{mmol})$ after purification by $\mathrm{SiO}_{2}$ column chromatography $\left(\mathrm{CHCl}_{3}:\right.$ Hexane, 3:1) yielded 6k (388mg, 64\%) as yellow oil.

Following GP7 with 1-benzyl-2-(4-chlorophenyl)-4,5dihydro- $1 H$-imidazole $\mathbf{1 i}(1.1 \mathrm{mmol})$ yielded $\mathbf{6 k}$ (407mg, 61\%).

${ }^{1} \mathrm{H}$ NMR $\left(\mathrm{CDCl}_{3}, 600 \mathrm{MHz}\right): \delta 3.31(\mathrm{~s}, 3 \mathrm{H}), 3.81(\mathrm{~s}, 3 \mathrm{H}), 3.82(\mathrm{t}, 2 \mathrm{H}, J=7.6 \mathrm{~Hz}), 4.10(\mathrm{t}, 2 \mathrm{H}$, $J=6.8 \mathrm{~Hz}), 4.64$ (s, $2 \mathrm{H}), 6.99$ (dd, 1H, J=7.8, $1.8 \mathrm{~Hz}), 7.19$ (t, 1H, J=8.1 Hz), 7.24- 7.28 (m, $2 \mathrm{H})$, 7.34 (d, 3H, J=6.1 Hz), 7.37 - 7.41 (m, 2 H), 7.43 (d, 2H, J=8.6 Hz), 7.50 (br., $1 \mathrm{H}), 7.70$ (d, 3H, 
$J=8.6 \mathrm{~Hz}) \mathrm{ppm} .{ }^{13} \mathrm{C}\{1 \mathrm{H}\} \mathrm{NMR}\left(\mathrm{CDCl}_{3}, 150 \mathrm{MHz}\right): \delta 47.8,48.9,51.6,52.0,52.3,115.3,117.8$, 119.9, 123.0, 125.5, 127.9 (2C), 128.3, 129.0 (2C), 129.3 (2C), 129.7, 129.9, 130.7 (2C), 132.4, 134.5, 136.6, 137.6, 140.2, 140.8, 155.1, 162.6, 164.2, 187.2 ppm. MS (ESI): m/z = 630 [M+Na$\mathrm{H}]^{+}$. HRMS (TOF ES ${ }^{+}$): m/z [M-H] $]^{+}$calculated for $\mathrm{C}_{31} \mathrm{H}_{27} \mathrm{Cl}_{2} \mathrm{~N}_{3} \mathrm{O}_{6}$ : 606.1204; found: 606.1211. $\mathrm{R}_{\mathrm{f}}\left(\mathrm{CHCl}_{3}: \mathrm{MeOH}, 100: 1\right)=0.4$. IR (KBr plate): 753, 776, 1063, 1218, 1269, 1483, 1535, 1590, $1638,1666,1724,3379 \mathrm{~cm}^{-1}$.

Dimethyl 1-(2-\{benzyl[(3-chlorophenyl)carbamoyl]amino\}ethyl)-2-(thiophen-2-ylcarbonyl)$1 H$-pyrrole-3,4-dicarboxylate (61)

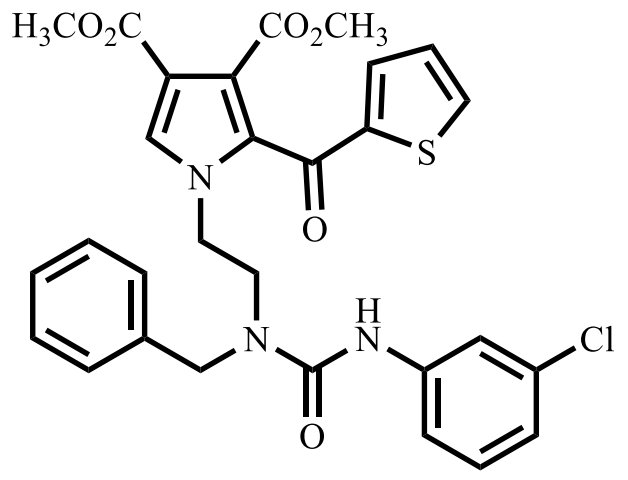

Following GP6 with methyl (2E)-3-[3-benzyl-2-(3methoxy-3-oxoprop-1-yn-1-yl)-2-(thiophen-2-yl) imidazolidin-1-yl]prop-2-enoate $\mathbf{3 k}(1 \mathrm{mmol})$ and 3 chlorophenyl isocyanate $(1.1 \mathrm{mmol})$ after purification by $\mathrm{SiO}_{2}$ column chromatography $\left(\mathrm{CHCl}_{3}:\right.$ Hexane, 3:1) yielded $\mathbf{6 1}(365 \mathrm{mg}, 63 \%)$ as colorless oil.

Following GP7 with 1-benzyl-2-(thiophen-2-yl)-4,5dihydro- $1 H$-imidazole (1.1 mmol) yielded 61 (318mg, 50\%).

${ }^{1} \mathrm{H} \mathrm{NMR}\left(\mathrm{CDCl}_{3}, 600 \mathrm{MHz}\right): \delta 3.42$ (s, $\left.3 \mathrm{H}\right), 3.78$ - 3.84 (m, $\left.2 \mathrm{H}\right), 3.81$ (s, $\left.3 \mathrm{H}\right), 3.96$ - 4.05 (m, 2 H), $4.63(\mathrm{~s}, 2 \mathrm{H}), 6.98(\mathrm{~d}, 1 \mathrm{H}, J=8.1 \mathrm{~Hz}), 7.10(\mathrm{t}, 1 \mathrm{H}, J=4.0 \mathrm{~Hz}), 7.19$ (t, 1H, J=8.1 Hz), 7.23 (s, 1 H), 7.29 - 7.35 (m, $3 \mathrm{H}), 7.37$ (t, 2H, J=7.3 Hz), 7.56 (d, 2H, J=4.0 Hz), 7.73 (s, $1 \mathrm{H}), 7.77$ (d, 1H, $J=5.0 \mathrm{~Hz}), 7.87$ (br., $1 \mathrm{H}) \mathrm{ppm} .{ }^{13} \mathrm{C}\{1 \mathrm{H}\} \mathrm{NMR}\left(\mathrm{CDCl}_{3}, 150 \mathrm{MHz}\right): \delta 47.5,48.8,51.5,52.0,52.2$, 115.3, 117.8, 119.9, 122.9, 123.8, 127.9 (2C), 128.2, 128.3, 129.2 (2C), 129.8, 130.4, 131.6, 134.4, 135.1, 136.1, 137.7, 140.8, 144.0, 155.0, 162.7, 164.4, 179.9 ppm. MS (ESI): m/z = $580[\mathrm{M}+\mathrm{H}]^{+}$. HRMS (TOF ES ${ }^{+}$): $\mathrm{m} / \mathrm{z}[\mathrm{M}-\mathrm{H}]^{+}$calculated for $\mathrm{C}_{29} \mathrm{H}_{26} \mathrm{ClN}_{3} \mathrm{O}_{6} \mathrm{~S}: 578.1158$; found: 578.1169. $\mathrm{R}_{\mathrm{f}}$ $\left(\mathrm{CHCl}_{3}: \mathrm{MeOH}, 100: 1\right)=0.6$. IR (KBr plate): 1210, 1270, 1415, 1482, 1531, 1593, 1724, 1737, $3376 \mathrm{~cm}^{-1}$.

\section{tret-Butyl [2-(3,4-diacetyl-2-benzoyl-1H-pyrrol-1-yl)ethyl]methylcarbamate (6n)}

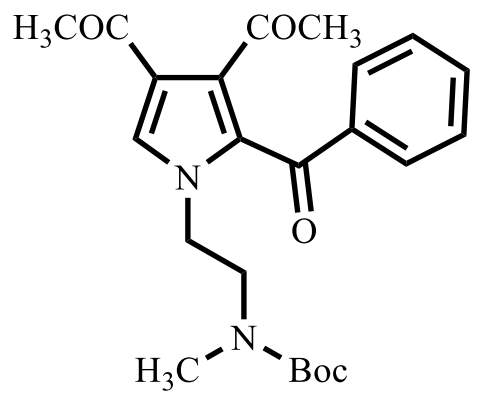

Following GP6 with (3E)-4-[3-methyl-2-(3-oxobut-1-yn-1-yl)-2phenylimidazolidin-1-yl]but-3-en-2-one 3o $(1 \mathrm{mmol})$ and di-tertbutyl dicarbonate $(1.1 \mathrm{mmol})$ after purification by $\mathrm{SiO}_{2}$ column chromatography $\left(\mathrm{CHCl}_{3}: \mathrm{Hexane}, 3: 1\right)$ yielded 6n (164mg, 40\%) as yellow solid.

Following GP7 with 1-methyl-2-phenyl-4,5-dihydro-1H-imidazole 1f $(1.1 \mathrm{mmol})$ yielded 6n (131 mg, 29\%). 
${ }^{1} \mathrm{H}$ NMR $\left(\mathrm{CDCl}_{3}, 600 \mathrm{MHz}\right.$, a mixture of rotamers in a ratio $\left.\sim 0.4: 0.6\right): \delta 1.37$ (br., $\left.3.6 \mathrm{H}\right), 1.43$ (br, $5.4 \mathrm{H}), 2.18$ (s, $3 \mathrm{H}), 2.40$ (s, $3 \mathrm{H}), 2.74$ (br., $3 \mathrm{H}), 3.52$ - 3.65 (m, 2H), 4.17 - 4.27 (m, 0.8 H), 4.27 - 4.37 (m, 1.2 H), 7.23 (br., 0.4 H), 7.34 (br., 0.6 H), 7.38 - 7.43 (m, 2 H), 7.52 - 7.57 $(\mathrm{m}, 1 \mathrm{H}), 7.62-7.73(\mathrm{~m}, 2 \mathrm{H}) \mathrm{ppm} .{ }^{13} \mathrm{C}\{1 \mathrm{H}\} \mathrm{NMR}\left(\mathrm{CDCl}_{3}, 150 \mathrm{MHz}\right)$ : major rotamer $\delta 27.8$, 28.5 (3C), 31.6, 35.6, 46.8, 49.7, 80.2, 123.1, 128.6 (2 C), 129.3 (2 C), 131.1, 131.7, 133.1, 133.4, 138.7, 155.8, 188.6, 192.1, $199.6 \mathrm{ppm}$; minor rotamer $\delta$ 27.8, 28.5 (3C), 31.6, 34.8, 47.3, 50.3, 80.3, 123.4, 128.6 (2 C), 129.3 (2 C), 130.7, 131.7, 132.9, 133.4, 138.6, 155.3, 188.4, 192.1, 199.2 ppm. MS (ESI): $\mathrm{m} / \mathrm{z}=435[\mathrm{M}+\mathrm{Na}]^{+}$. HRMS $\left(\mathrm{TOF} \mathrm{ES}^{+}\right): \mathrm{m} / \mathrm{z}[\mathrm{M}+\mathrm{H}]^{+}$calculated for $\mathrm{C}_{23} \mathrm{H}_{28} \mathrm{~N}_{2} \mathrm{O}_{5}$ : 413.2071; found: 413.2062. m.p. $121-122^{\circ} \mathrm{C}$. IR ( $\mathrm{KBr}$ plate): 701, 771, 911, 1069, 1161, 1217 , $1274,1401,1649,1701,1727,2958 \mathrm{~cm}^{-1}$.

\section{1-Benzyl-3-(3-chlorophenyl)-1-\{2-[3,4-diacetyl-2-(4-chlorobenzoyl)-1H-pyrrol-1- yl]ethyl\}urea (6o)}

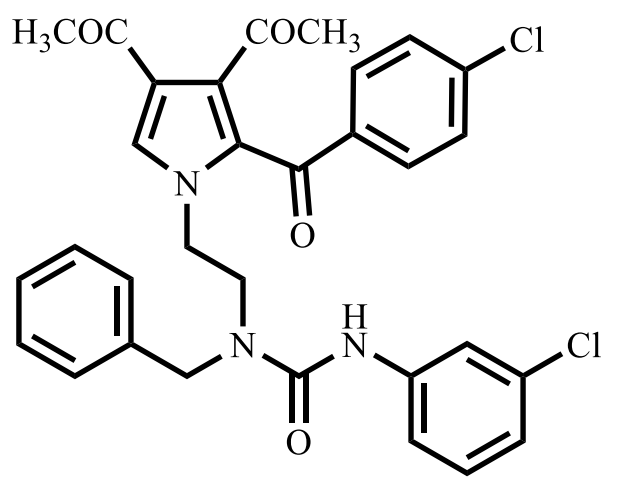

Following GP6 with (3E)-4-[3-benzyl-2-(4-chlorophenyl)2-(3-oxobut-1-yn-1-yl)imidazolidin-1-yl]but-3-en-2-one 3q $(1 \mathrm{mmol})$ and 3-chlorophenyl isocyanate $(1.1 \mathrm{mmol})$ after purification by $\mathrm{SiO}_{2}$ column chromatography $\left(\mathrm{CHCl}_{3}:\right.$ Hexane, 3:1) yielded $\mathbf{6 0}(260 \mathrm{mg}, 45 \%)$ as yellow oil.

Following GP7 with 1-benzyl-2-(4-chlorophenyl)-4,5dihydro- $1 H$-imidazole $\mathbf{1 i}$ (1.1 mmol) yielded $\mathbf{6 o}(221 \mathrm{mg}, 35 \%)$.

${ }^{1} \mathrm{H}$ NMR $\left(\mathrm{CDCl}_{3}, 600 \mathrm{MHz}\right): \delta 2.19$ (s, $\left.3 \mathrm{H}\right), 2.41(\mathrm{~s}, 3 \mathrm{H}), 3.86(\mathrm{t}, 2 \mathrm{H}, J=7.3 \mathrm{~Hz}), 4.12(\mathrm{t}, 2 \mathrm{H}$, $J=6.8 \mathrm{~Hz}), 4.63$ (s, $2 \mathrm{H}), 6.99(\mathrm{dd}, 1 \mathrm{H}, J=8.3,1.8 \mathrm{~Hz}), 7.13-7.20$ (m, $2 \mathrm{H}), 7.31-7.37$ (m, $4 \mathrm{H})$, 7.38 - 7.43 (m, $4 \mathrm{H}), 7.48$ (br., $1 \mathrm{H}), 7.63$ (s, $1 \mathrm{H}), 7.65$ (d, 2H, J=8.6 Hz) ppm. ${ }^{13} \mathrm{C}\{1 \mathrm{H}\} \mathrm{NMR}$ $\left(\mathrm{CDCl}_{3}, 150 \mathrm{MHz}\right): \delta 28.0,31.7,47.6,49.2,51.8,117.6,119.8,123.2,123.8,127.8$ (2C), 128.4, 129.2 (2C), 129.3 (2C), 130.0, 130.0, 130.9 (2C), 131.8, 133.7, 134.6, 136.5, 137.4, 140.5, 140.6, 155.1, 188.1, 191.8, 199.2 ppm. MS (ESI): m/z = $598[\mathrm{M}+\mathrm{Na}-\mathrm{H}]^{+}$. HRMS $\left(\mathrm{TOF} \mathrm{ES}^{+}\right): \mathrm{m} / \mathrm{z}[\mathrm{M}-$ $\mathrm{H}]^{+}$calculated for $\mathrm{C}_{31} \mathrm{H}_{27} \mathrm{Cl}_{2} \mathrm{~N}_{3} \mathrm{O}_{4}$ : 574.1306; found: 574.1314. $\mathrm{R}_{\mathrm{f}}$ (Ethyl acetate: Hexane, 1:1) = 0.30. IR (KBr plate): $1195,1251,1381,1483,1528,1589,1666,3379 \mathrm{~cm}^{-1}$. 
yl]ethyl\}urea (6p)<smiles>CC(=O)c1cn(CCN(Cc2ccccc2)C(=O)Nc2cccc(Cl)c2)c(C(=O)c2cccs2)c1C(C)=O</smiles>

Following GP6 with (3E)-4-[3-benzyl-2-(3-oxobut-1-yn-1yl)-2-(thiophen-2-yl)imidazolidin-1-yl]but-3-en-2-one $\quad \mathbf{3 r}$ $(1 \mathrm{mmol})$ and 3-chlorophenyl isocyanate $(1.1 \mathrm{mmol})$ after purification by $\mathrm{SiO}_{2}$ column chromatography $\left(\mathrm{CHCl}_{3}:\right.$ Hexane, $\left.3: 1\right)$ yielded $\mathbf{6 p}(280 \mathrm{mg}, 51 \%)$ as colorless oil.

Following GP7 with 1-benzyl-2-(thiophen-2-yl)-4,5dihydro- $1 H$-imidazole (1.1 mmol) yielded 6p (235mg, 39\%).

${ }^{1} \mathrm{H} \mathrm{NMR}\left(\mathrm{CDCl}_{3}, 600 \mathrm{MHz}\right): \delta 2.23(\mathrm{~s}, 3 \mathrm{H}), 2.39(\mathrm{~s}, 3 \mathrm{H}), 3.85(\mathrm{t}, 2 \mathrm{H}, J=7.1 \mathrm{~Hz}), 4.04(\mathrm{t}, 2 \mathrm{H}$, $J=7.1 \mathrm{~Hz}), 4.63(\mathrm{~s}, 2 \mathrm{H}), 6.98(\mathrm{dd}, 1 \mathrm{H}, J=8.1,1.5 \mathrm{~Hz}), 7.06-7.09(\mathrm{~m}, 1 \mathrm{H}), 7.10(\mathrm{~s}, 1 \mathrm{H}), 7.18$ (t, $1 \mathrm{H}, J=8.1 \mathrm{~Hz}), 7.32-7.35(\mathrm{~m}, 3 \mathrm{H}), 7.37-7.40(\mathrm{~m}, 2 \mathrm{H}), 7.42$ (d, 1H, $J=7.1 \mathrm{~Hz}), 7.50$ (dd, 1H, J=4.0, $1.0 \mathrm{~Hz}), 7.59$ (br, $1 \mathrm{H}), 7.67(\mathrm{~s}, 1 \mathrm{H}), 7.76(\mathrm{dd}, 1 \mathrm{H}, J=5.0,1.0 \mathrm{~Hz}) \mathrm{ppm} .{ }^{13} \mathrm{C}\{1 \mathrm{H}\} \mathrm{NMR}\left(\mathrm{CDCl}_{3}\right.$, $150 \mathrm{MHz}): \delta 28.1,32.1,47.2,49.0,51.7,117.7,119.8,123.0,123.94,127.9$ (2C), 128.3, 128.5, 129.2 (2C), 129.9, 130.4, 131.3, 132.3, 134.5, 136.2, 136.5, 137.6, 140.7, 143.9, 155.1, 180.6, 192.0, 199.3 ppm. HRMS (TOF $\mathrm{ES}^{+}$): $\mathrm{m} / \mathrm{z}[\mathrm{M}-\mathrm{H}]^{+}$calculated for $\mathrm{C}_{29} \mathrm{H}_{26} \mathrm{ClN}_{3} \mathrm{O}_{4} \mathrm{~S}: 546.1260$; found:546.1269. $\mathrm{R}_{\mathrm{f}}\left(\mathrm{CHCl}_{3}: \mathrm{MeOH}, 100: 1\right)=0.3$. IR (KBr plate): 1212, 1273, 1419, 1484, 1532, $1593,1726,1739,3378 \mathrm{~cm}^{-1}$. 


\section{Reference}

[1] Gong, J.-F.; Fan, X.-H.; Xu, Ch.; Li, J.-L.; Wu, Y.-J.; Song M.-P. J. Organomet. Chem. 2007, 2006.

[2] Fujioka, H.; Murai, K.; Ohba, Y.; Hirose, H.; Kita, Y. Chem. Commun. 2006, 832-834.

[3] Anderson, M.W.; Begley, M.J.; Jones, R.C.F.; Saunders, J. J. Chem. Soc. Trans. 1984, 2599.

[4] Owen, A. Davis, O.A.; Hughes, M.; Bull, J.A. J. Org. Chem. 2013, 3470.

[5] Geden, J.V.; Pancholi A.K.; Shipman, M. J. Org. Chem. 2013, 4158.

[6] Golubenkova, A.S.; Golantsov, N.E.; Festa, A.A.; Voskressensky, L.G. Molbank 2020, M1137. 


\section{Crystal Data and Structural Refinement}

\subsection{Crystal data and structural refinement for imidazolidine $3 \mathrm{~g}$}

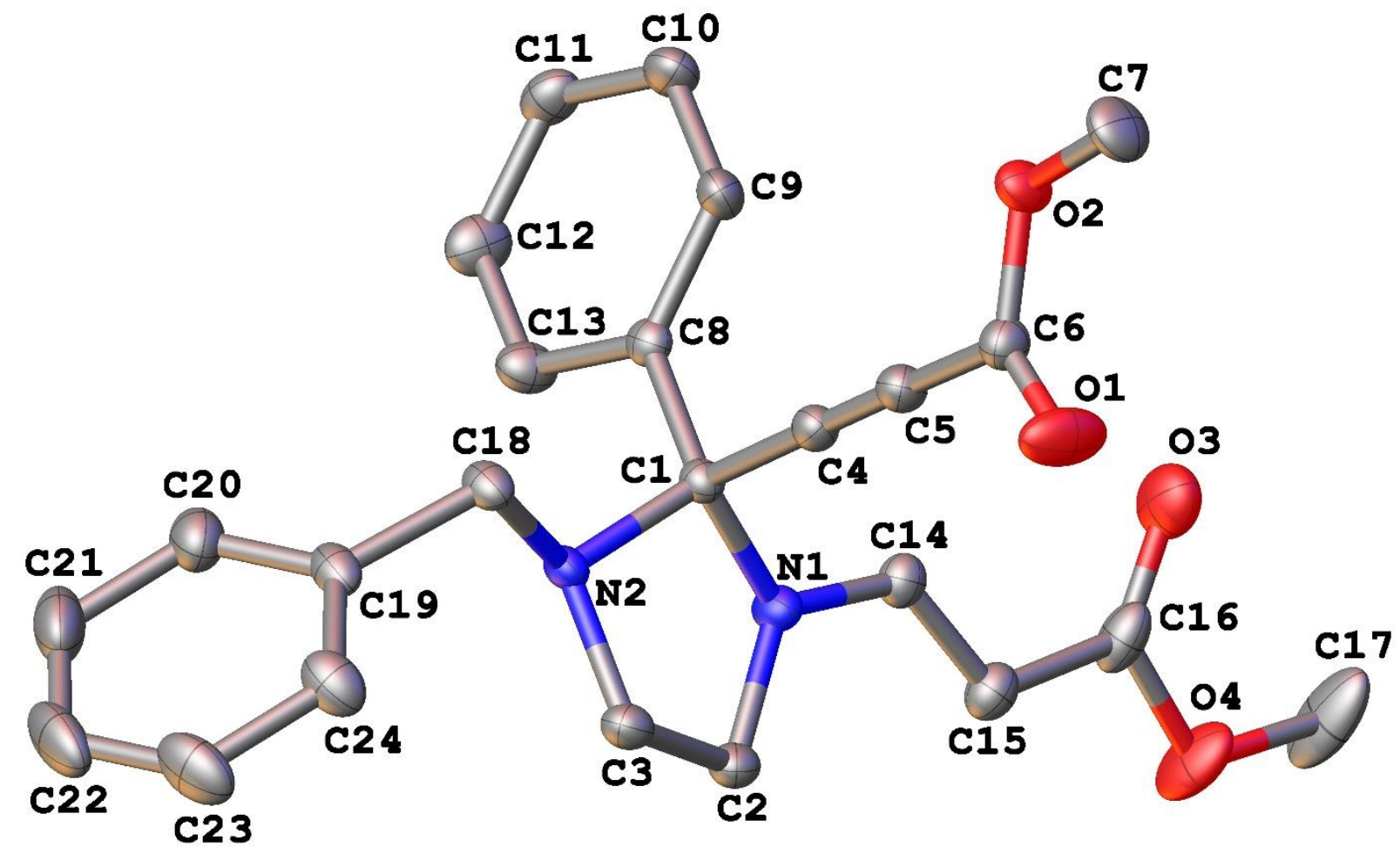

Figure S1. A thermal ellipsoid plot for the crystal structure 3g. The ellipsoid contour is shown with $50 \%$ probability level

The crystal of $\mathbf{3 g}$ for X-ray diffraction study has been obtained through the dissolving of compound in ether, followed by slow evaporation of the solvent at room temperature. Single crystals of $\mathrm{C}_{24} \mathrm{H}_{24} \mathrm{~N}_{2} \mathrm{O}_{4}$ were prisms. X-ray diffraction patterns was measured on a Bruker APEX-II CCD diffractometer. The crystal was kept at $120 \mathrm{~K}$ during data collection.

Table S1. Crystal data and structure refinement for $\mathbf{3 g}$

$\begin{array}{ll}\text { Identification code } & \mathbf{3 g} \\ \text { Empirical formula } & \mathrm{C}_{24} \mathrm{H}_{24} \mathrm{~N}_{2} \mathrm{O}_{4} \\ \text { Formula weight } & 404.45 \\ \text { Temperature/K } & 120 \\ \text { Crystal system } & \text { monoclinic } \\ \text { Space group } & \mathrm{P} 2{ }_{1} / \mathrm{c} \\ \mathrm{a} / \AA & 22.6809(19) \\ \mathrm{b} / \AA & 10.6711(10) \\ \mathrm{c} / \AA & 18.1453(17) \\ \alpha /{ }^{\circ} & 90 \\ \beta /{ }^{\circ} & 102.670(2) \\ \gamma /{ }^{\circ} & 90 \\ \text { Volume } / \AA^{3} & 4284.8(7) \\ \mathrm{Z} & 8\end{array}$




$\begin{array}{ll}\rho_{\text {calcg }} / \mathrm{cm}^{3} & 1.254 \\ \mu / \mathrm{mm}^{-1} & 0.086 \\ \mathrm{~F}(000) & 1712.0 \\ \text { Crystal size } / \mathrm{mm}^{3} & 0.24 \times 0.2 \times 0.15 \\ \text { Radiation } & \mathrm{MoK} \alpha(\lambda=0.71073) \\ 2 \Theta \text { range for data collection } /{ }^{\circ} & 3.682 \text { to } 58.26 \\ \text { Index ranges } & -23 \leq \mathrm{h} \leq 31,-14 \leq \mathrm{k} \leq 12,-24 \leq 1 \leq 14 \\ \text { Reflections collected } & 25284 \\ \text { Independent reflections } & 11479\left[\mathrm{R}_{\text {int }}=0.0293, \mathrm{R}_{\text {sigma }}=0.0409\right] \\ \text { Data/restraints/parameters } & 11479 / 0 / 545 \\ \text { Goodness-of-fit on } \mathrm{F}^{2} & 1.021 \\ \text { Final R indexes }[\mathrm{I}>=2 \sigma(\mathrm{I})] & \mathrm{R}_{1}=0.0457, \mathrm{wR}_{2}=0.1025 \\ \text { Final R indexes [all data }] & \mathrm{R}_{1}=0.0675, \mathrm{wR}_{2}=0.1142 \\ \text { Largest diff. peak } / \text { hole } / \mathrm{e} \AA^{-3} & 0.35 /-0.27\end{array}$

Atomic coordinates, bond lengths and angles and thermal parameters have been deposited at the Cambridge Crystallographic Data Center (CCDC), reference number 2000636.

\subsection{Crystal data and structural refinement for pyrrole $4 a$}

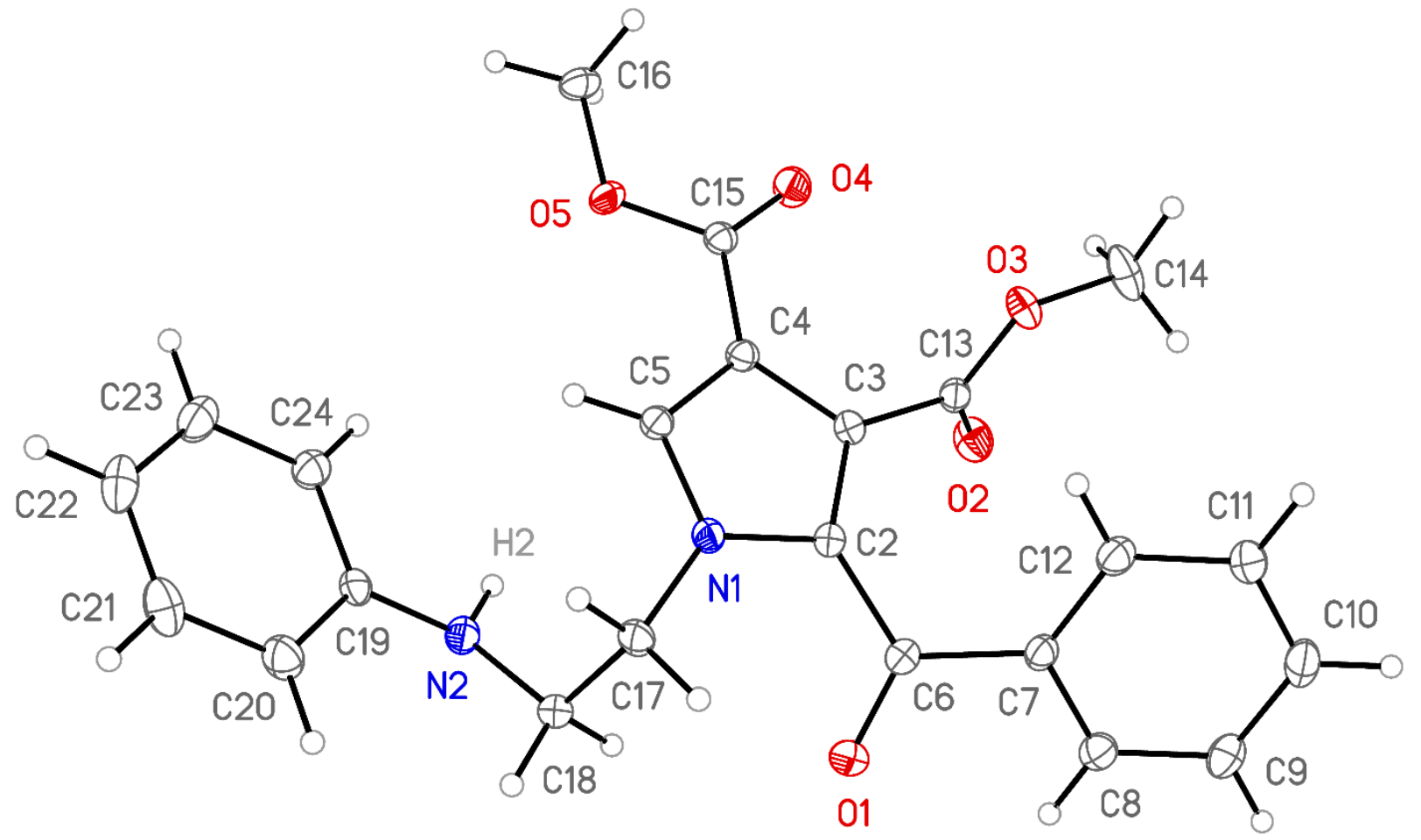

Figure S2. A thermal ellipsoid plot for the crystal structure 4a. The ellipsoid contour is shown with $50 \%$ probability level

The crystal of 4a for X-ray diffraction study has been obtained through the dissolving of compound in toluene, followed by slow evaporation of the solvent at room temperature. Yellow crystals of 
$\mathrm{C}_{23} \mathrm{H}_{22} \mathrm{~N}_{2} \mathrm{O}_{5}$ at $120 \mathrm{~K}$ were triclinic. X-ray diffraction data for $\mathbf{4 a}$ were collected on a Bruker APEX DUO diffractometer.

Table S2. Crystal data and structure refinement for $\mathbf{4 a}$

\begin{tabular}{|c|c|}
\hline Identification code & $\mathbf{4 a}$ \\
\hline Empirical formula & $\mathrm{C}_{23} \mathrm{H}_{22} \mathrm{~N}_{2} \mathrm{O} 5$ \\
\hline Formula weight & 406.42 \\
\hline Temperature/K & 120 \\
\hline Crystal system & Triclinic \\
\hline Space group & $\mathrm{P}-1$ \\
\hline $\mathrm{a} / \AA$ & $7.4566(4)$ \\
\hline $\mathrm{b} / \AA$ & $10.3873(6)$ \\
\hline $\mathrm{c} / \AA$ & $13.2455(7)$ \\
\hline$\alpha /^{\circ}$ & $90.3690(10)$ \\
\hline$\beta /{ }^{\circ}$ & $93.8990(10)$ \\
\hline$\gamma /{ }^{\circ}$ & $100.0230(10)$ \\
\hline Volume $/ \AA^{3}$ & $1000.02(9)$ \\
\hline $\mathrm{Z}$ & 2 \\
\hline$\rho_{\text {calc }} \mathrm{g} / \mathrm{cm}^{3}$ & 1.350 \\
\hline$\mu / \mathrm{mm}^{-1}$ & 0.096 \\
\hline $\mathrm{F}(000)$ & 428 \\
\hline Crystal size $/ \mathrm{mm}^{3}$ & $0.33 \times 0.3 \times 0.24$ \\
\hline Radiation & $\operatorname{MoK} \alpha(\lambda=0.71073)$ \\
\hline \multicolumn{2}{|c|}{$2 \Theta$ range for data collection $/{ }^{\circ} 2.007$ to 29.999} \\
\hline Index ranges & $-10 \leq \mathrm{h} \leq 10,-14 \leq \mathrm{k} \leq 14,-18 \leq 1 \leq 18$ \\
\hline Reflections collected & 13392 \\
\hline Independent reflections & $5846\left[\mathrm{R}_{\mathrm{int}}=0.0232\right]$ \\
\hline Data/restraints/parameters & $5846 / 0 / 277$ \\
\hline Goodness-of-fit on $\mathrm{F}^{2}$ & 0.990 \\
\hline Final $R$ indexes $[\mathrm{I}>=2 \sigma(\mathrm{I})]$ & $\mathrm{R}_{1}=0.0399, \mathrm{wR}_{2}=0.1058$ \\
\hline Final $R$ indexes [all data] & $\mathrm{R}_{1}=0.0502, \mathrm{wR}_{2}=0.1152$ \\
\hline Largest diff. peak/hole / e $\AA^{-3}$ & $0.393 /-0.231$ \\
\hline
\end{tabular}

Atomic coordinates, bond lengths and angles and thermal parameters have been deposited at the Cambridge Crystallographic Data Center (CCDC), reference number 2000635. 
7. ${ }^{1} \mathrm{H}$ NMR and ${ }^{13} \mathrm{C}$ NMR Spectra of Products

${ }^{1} \mathrm{H}$ NMR spectrum, $\mathrm{CDCl}_{3}, 600 \mathrm{MHZ}$

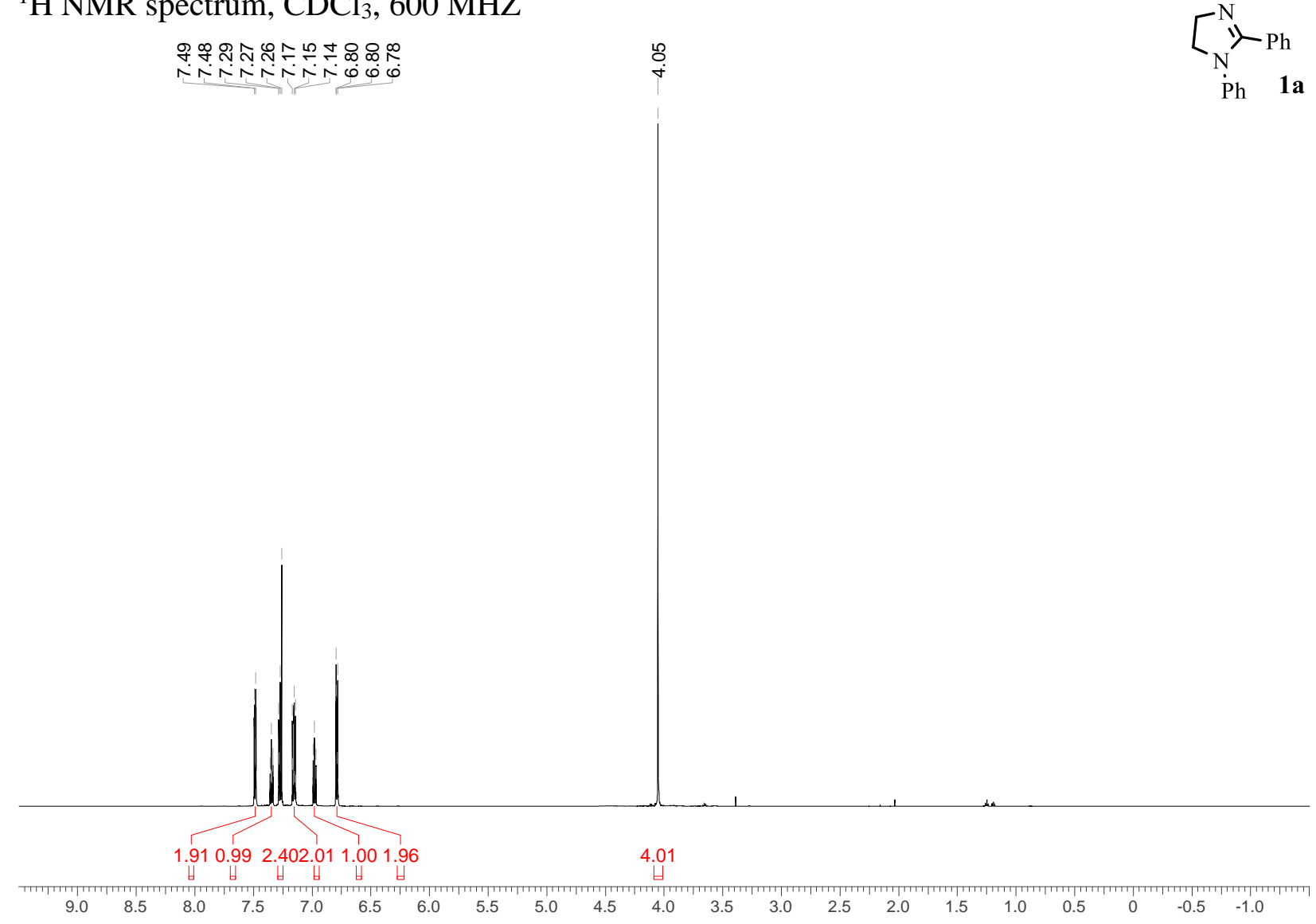

${ }^{13} \mathrm{C}$ NMR spectrum, $\mathrm{CDCl}_{3}, 150 \mathrm{MHz}$

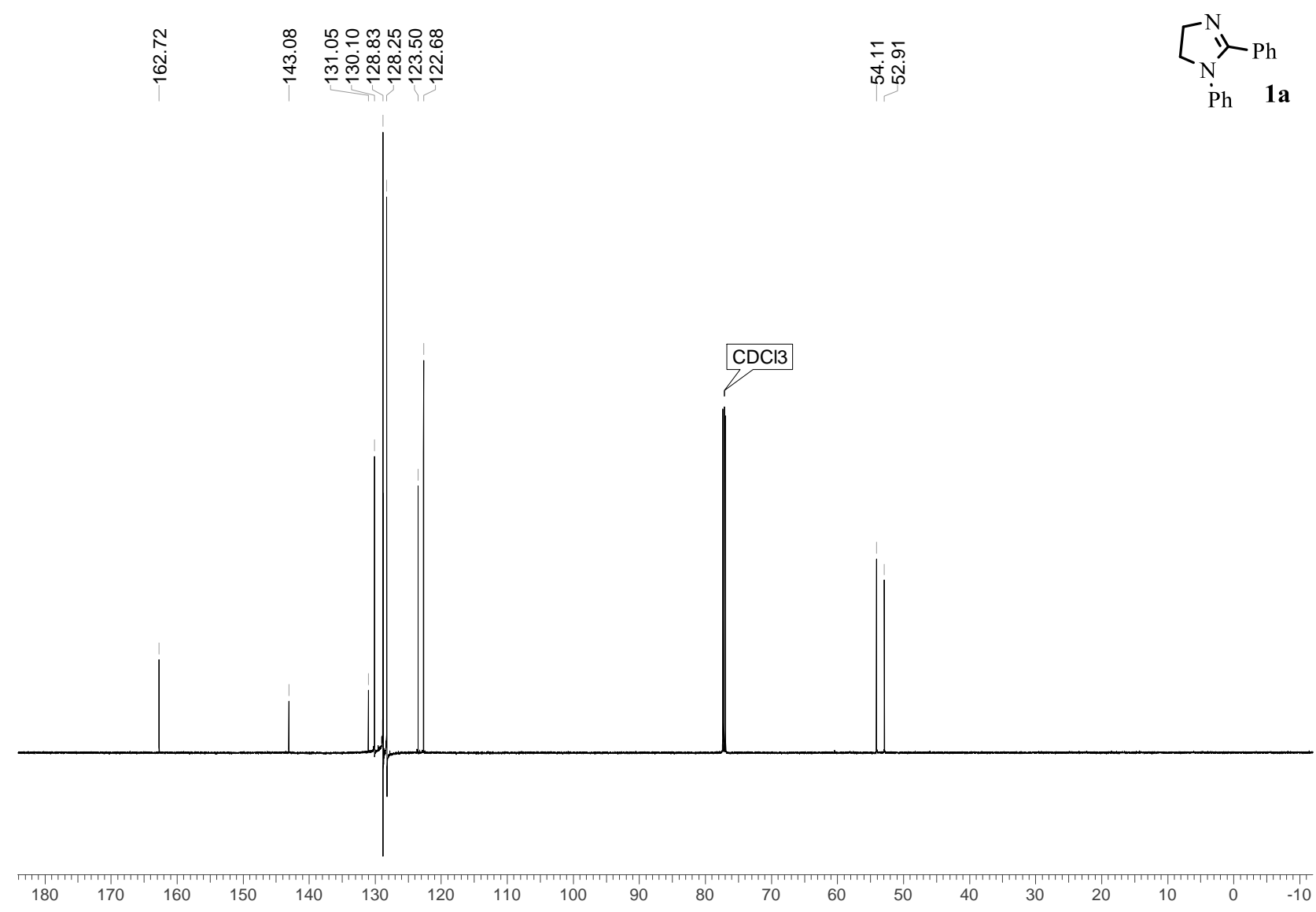


${ }^{1} \mathrm{H}$ NMR spectrum, $\mathrm{CDCl}_{3}, 600 \mathrm{MHZ}$

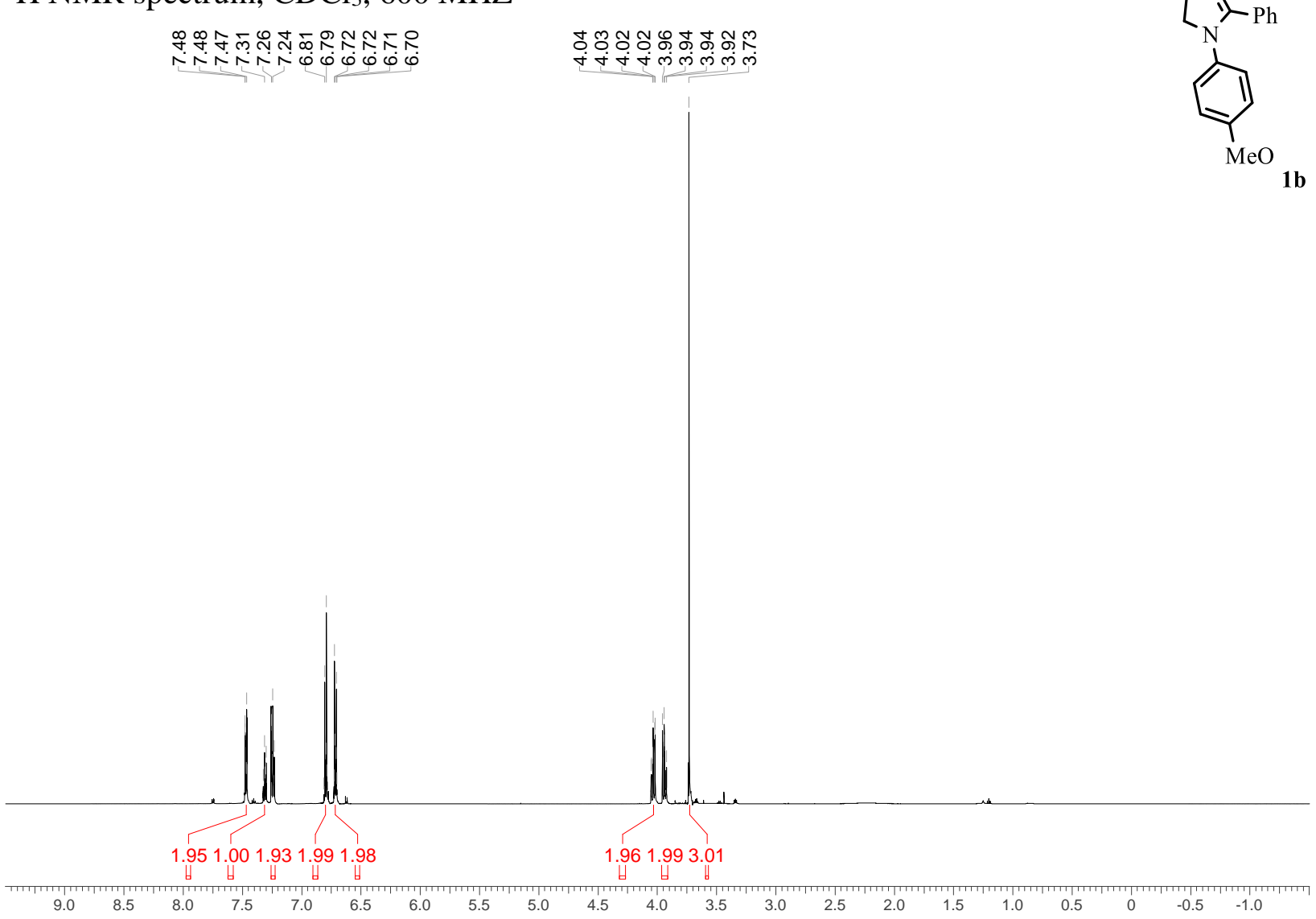

${ }^{13} \mathrm{C}$ NMR spectrum, $\mathrm{CDCl}_{3}, 150 \mathrm{MHz}$

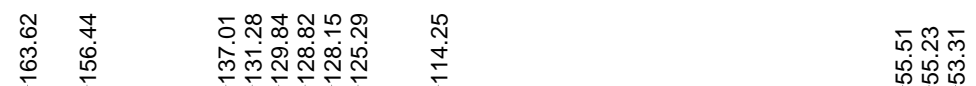
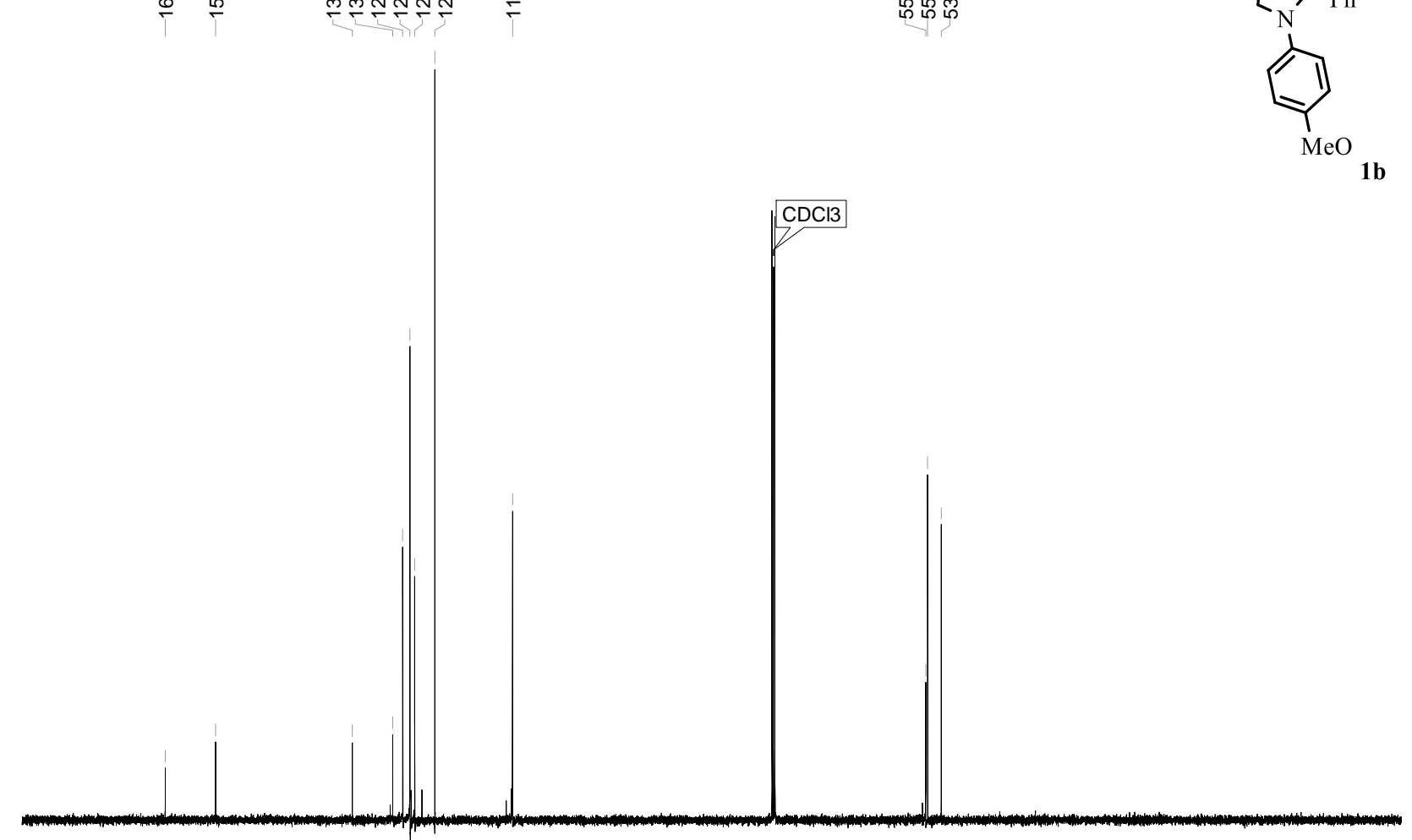

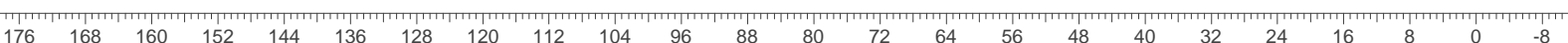


${ }^{1} \mathrm{H}$ NMR spectrum, $\mathrm{CDCl}_{3}, 600 \mathrm{MHZ}$

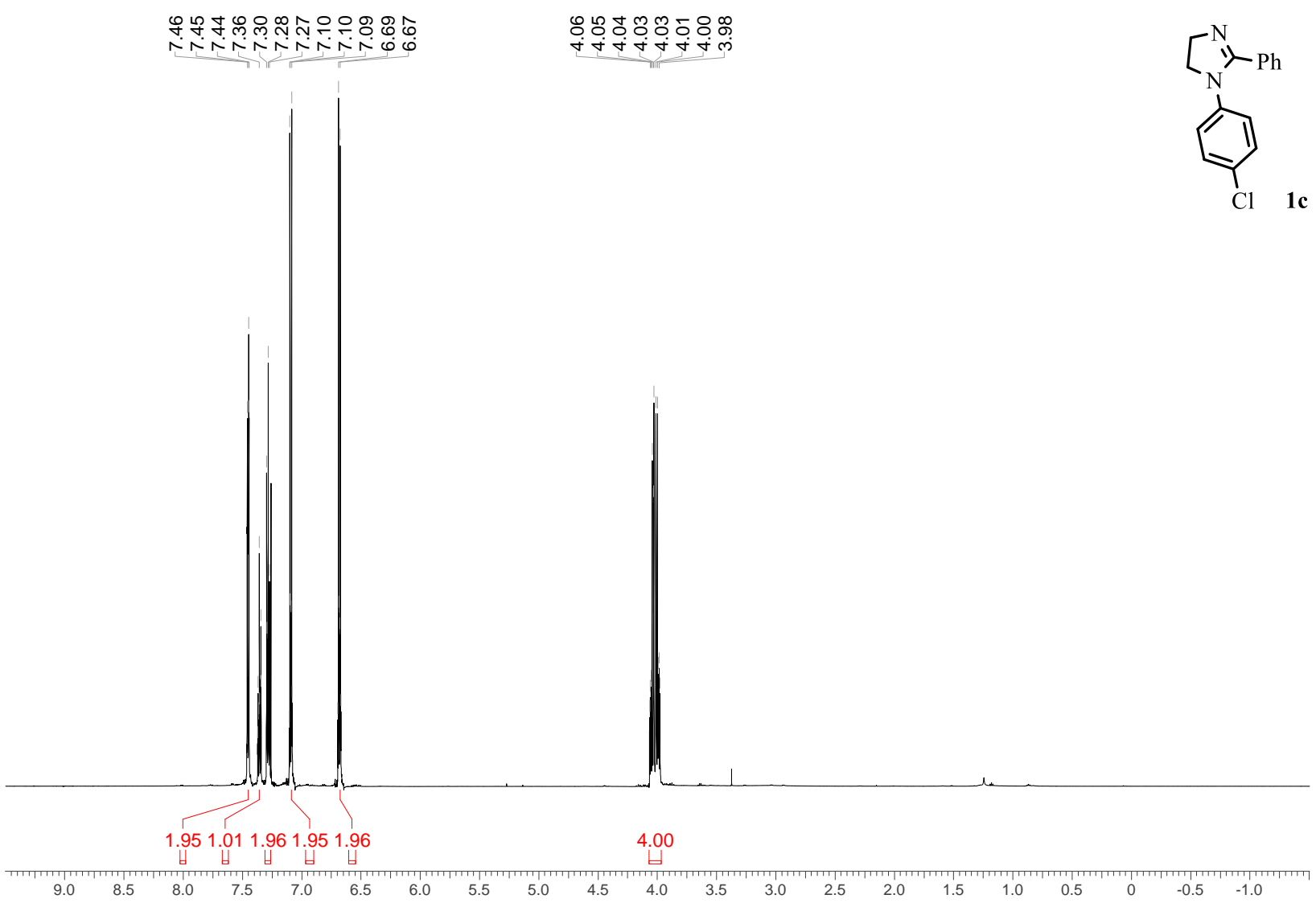

${ }^{13} \mathrm{C}$ NMR spectrum, $\mathrm{CDCl}_{3}, 150 \mathrm{MHz}$

กิ

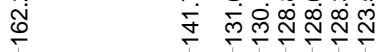

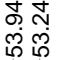

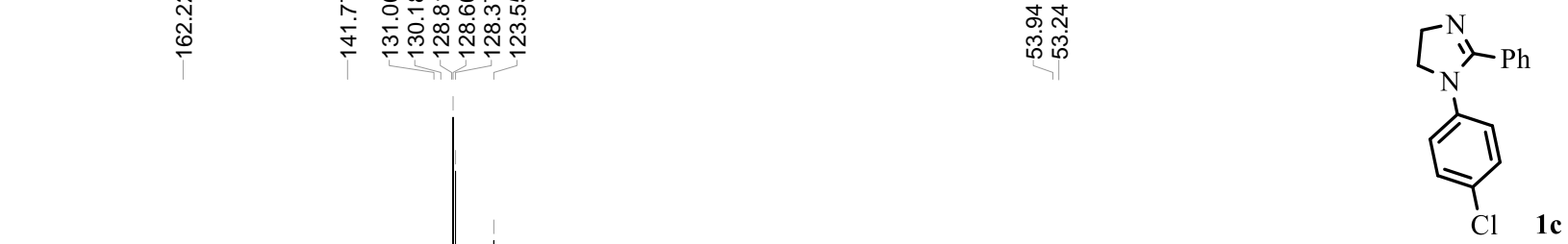

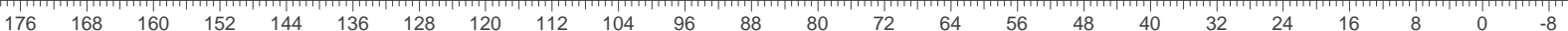


${ }^{1} \mathrm{H}$ NMR spectrum, $\mathrm{CDCl}_{3}, 600 \mathrm{MHZ}$

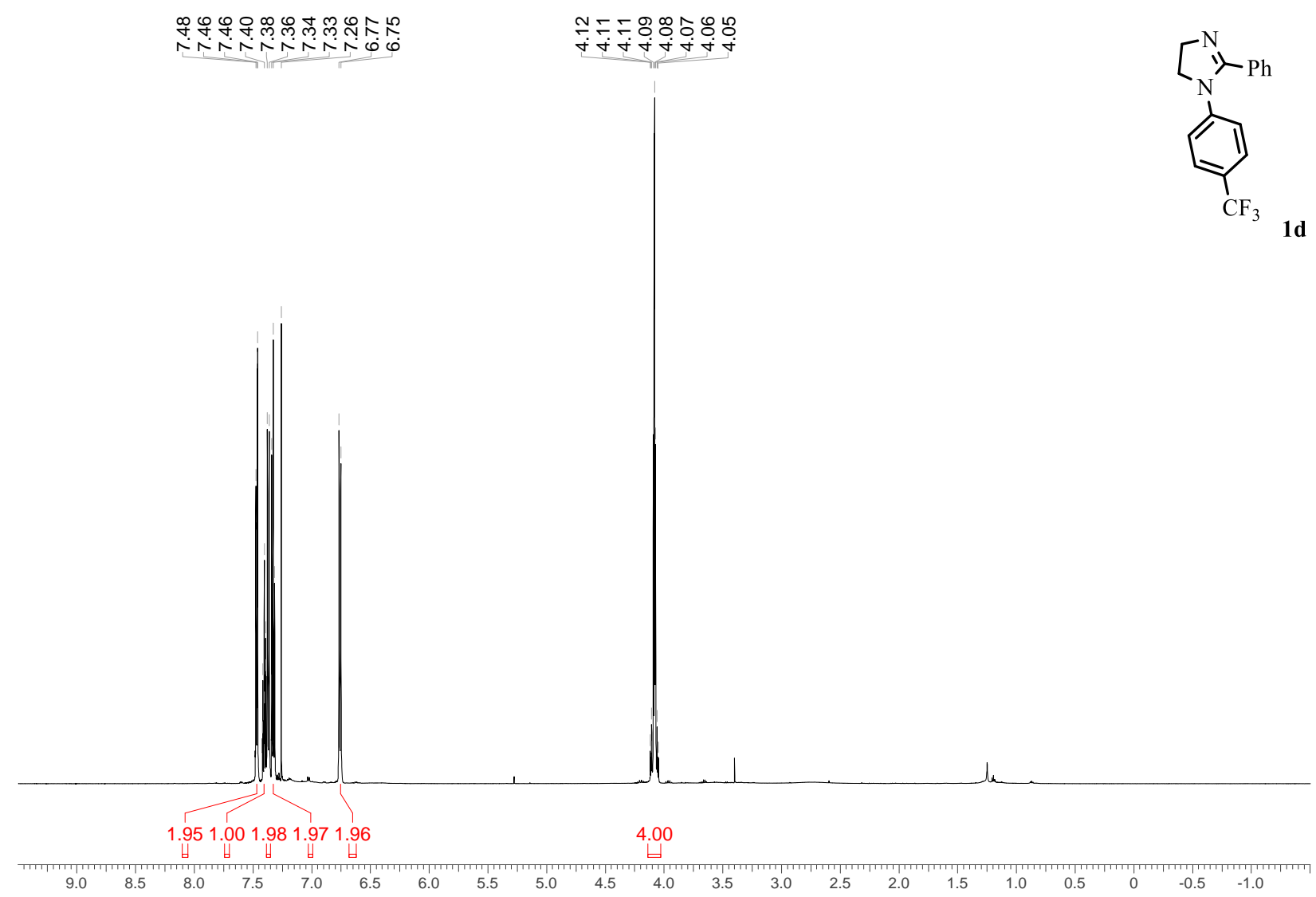

${ }^{13} \mathrm{C}$ NMR spectrum, $\mathrm{CDCl}_{3}, 150 \mathrm{MHz}$

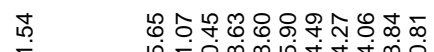

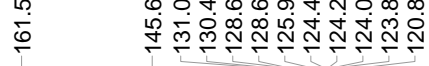

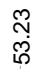

$\left[\mathrm{N}^{\mathrm{N}}-\mathrm{Ph}\right.$<smiles>Nc1ccc(C(F)(F)F)cc1</smiles>

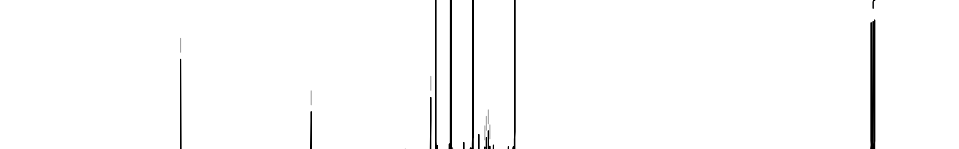

$\begin{array}{lllllllllll}176 & 168 & 160 & 152 & 144 & 136 & 128 & 120 & 112 & 104 & 96\end{array}$ 
${ }^{1} \mathrm{H}$ NMR spectrum, $\mathrm{CDCl}_{3}, 600 \mathrm{MHZ}$

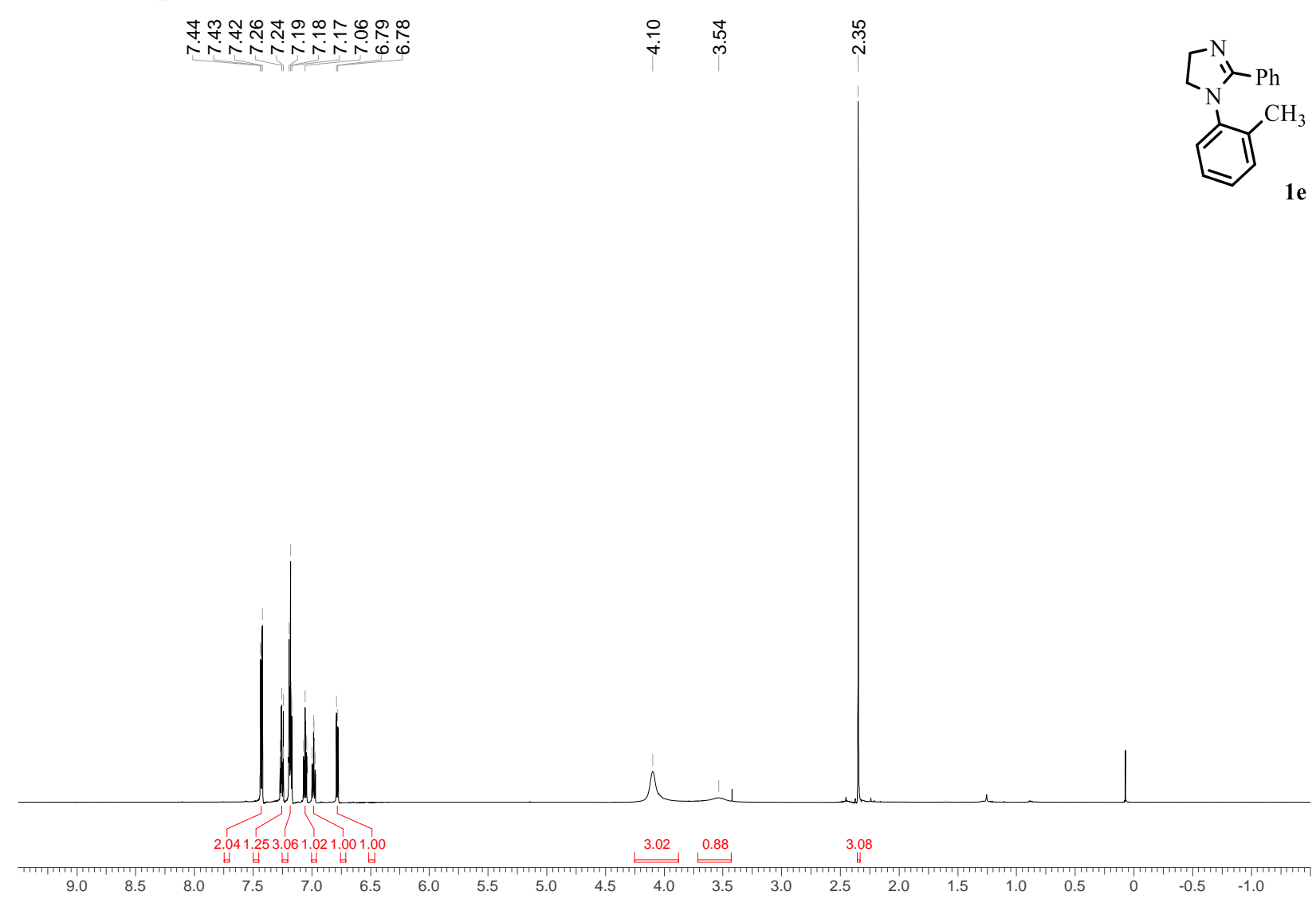

${ }^{13} \mathrm{C}$ NMR spectrum, $\mathrm{CDCl}_{3}, 150 \mathrm{MHz}$

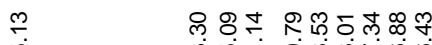

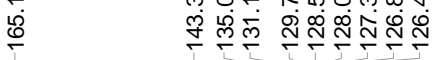

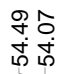

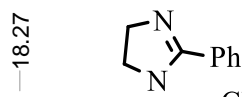

(1)

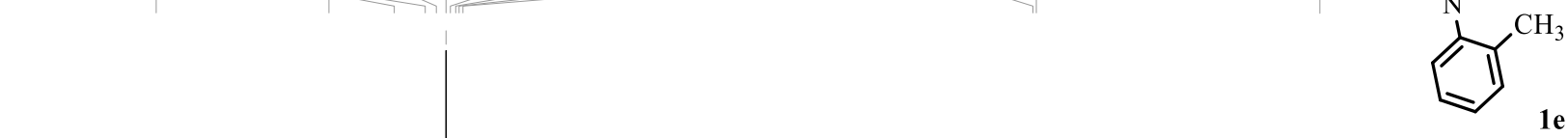

$\begin{array}{llllllllllllllllllllllll}176 & 168 & 160 & 152 & 144 & 136 & 128 & 120 & 112 & 104 & 96 & 88 & 80 & 72 & 64 & 56 & 48 & 40 & 32 & 24 & 16 & 8 & 0 & -8\end{array}$ 
${ }^{1} \mathrm{H}$ NMR spectrum, $\mathrm{CDCl}_{3}, 600 \mathrm{MHZ}$

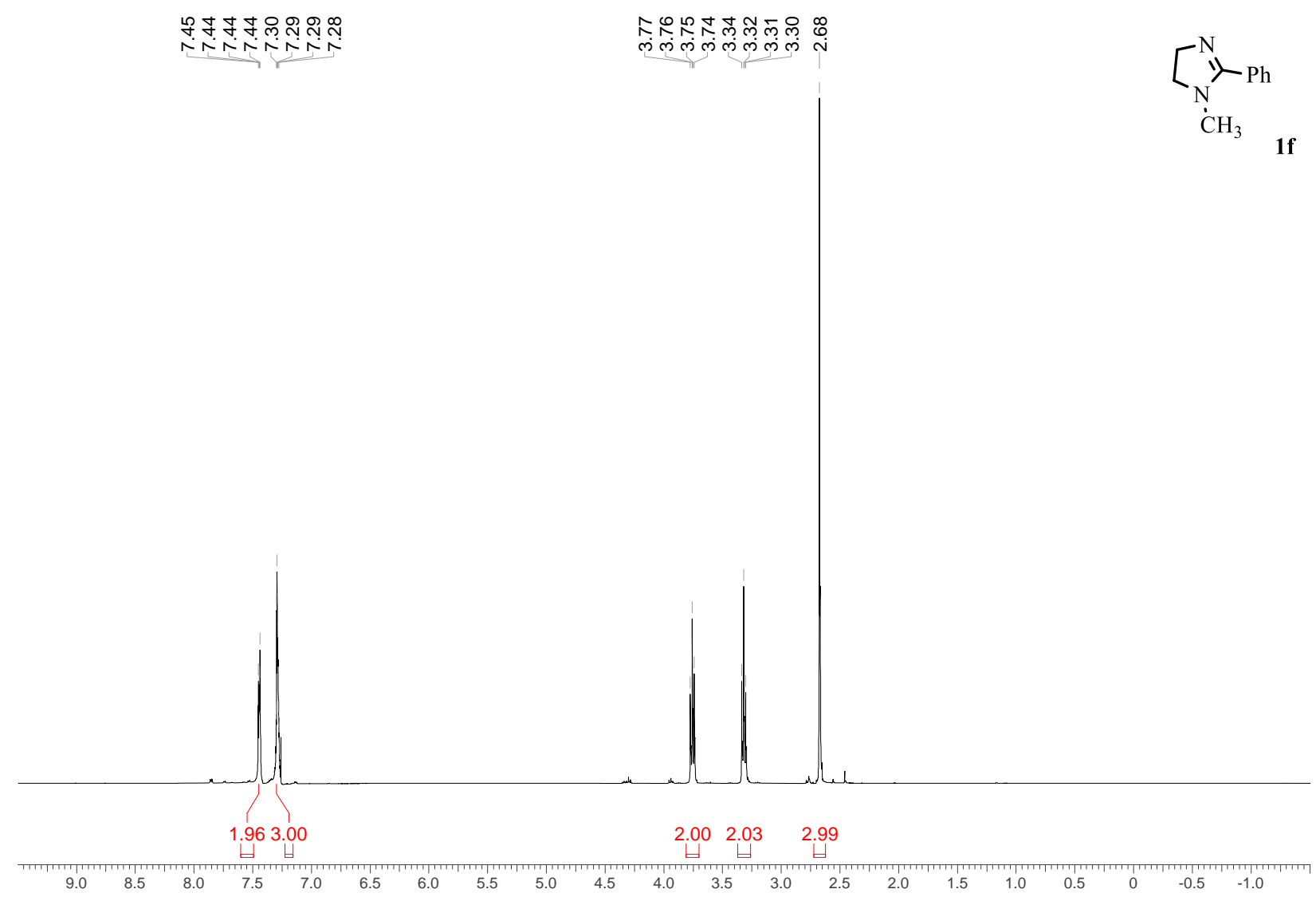

${ }^{13} \mathrm{C}$ NMR spectrum, $\mathrm{CDCl}_{3}, 150 \mathrm{MHz}$

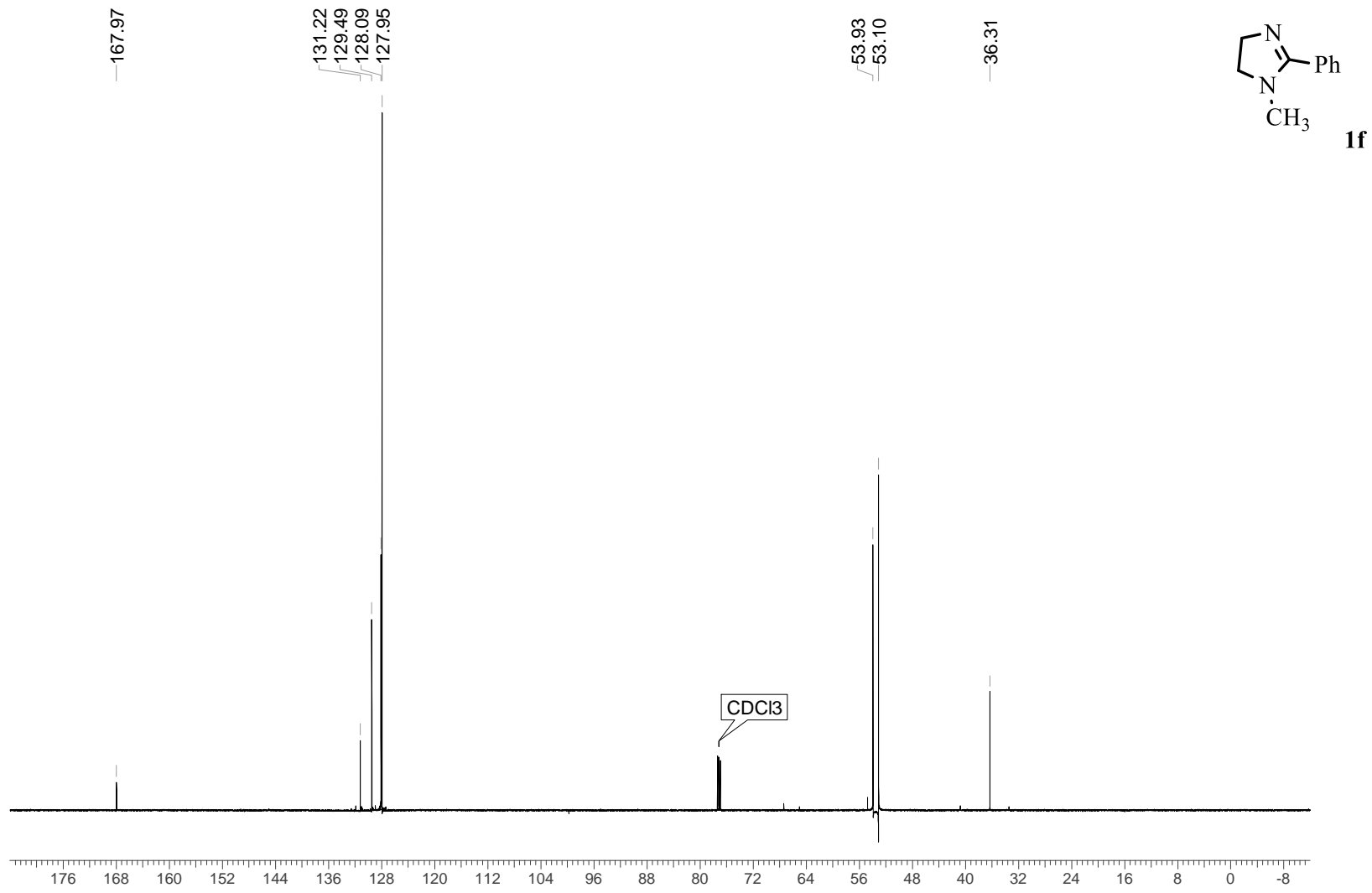


${ }^{1} \mathrm{H}$ NMR spectrum, $\mathrm{CDCl}_{3}, 600 \mathrm{MHZ}$

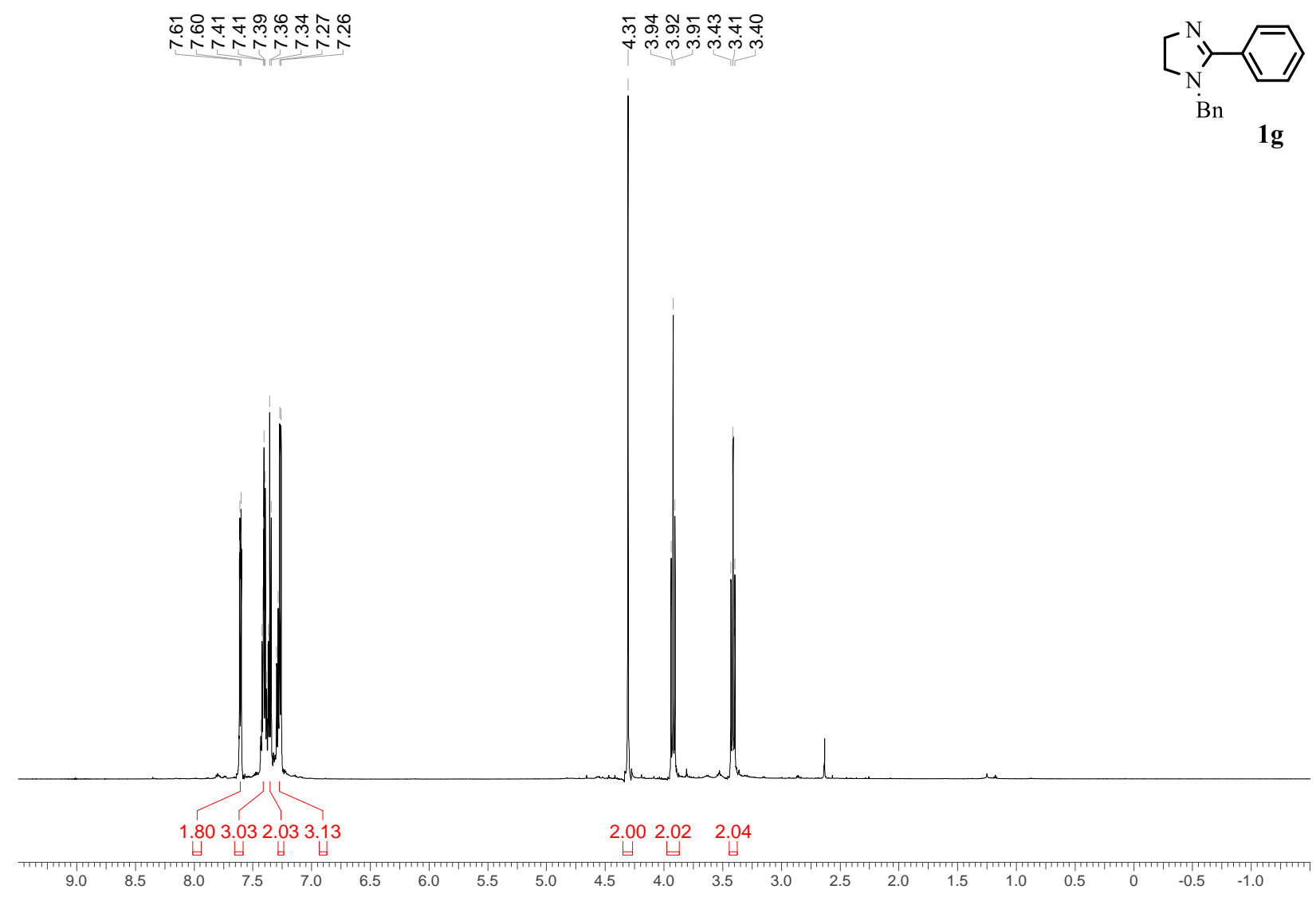

${ }^{13} \mathrm{C}$ NMR spectrum, $\mathrm{CDCl}_{3}, 150 \mathrm{MHz}$

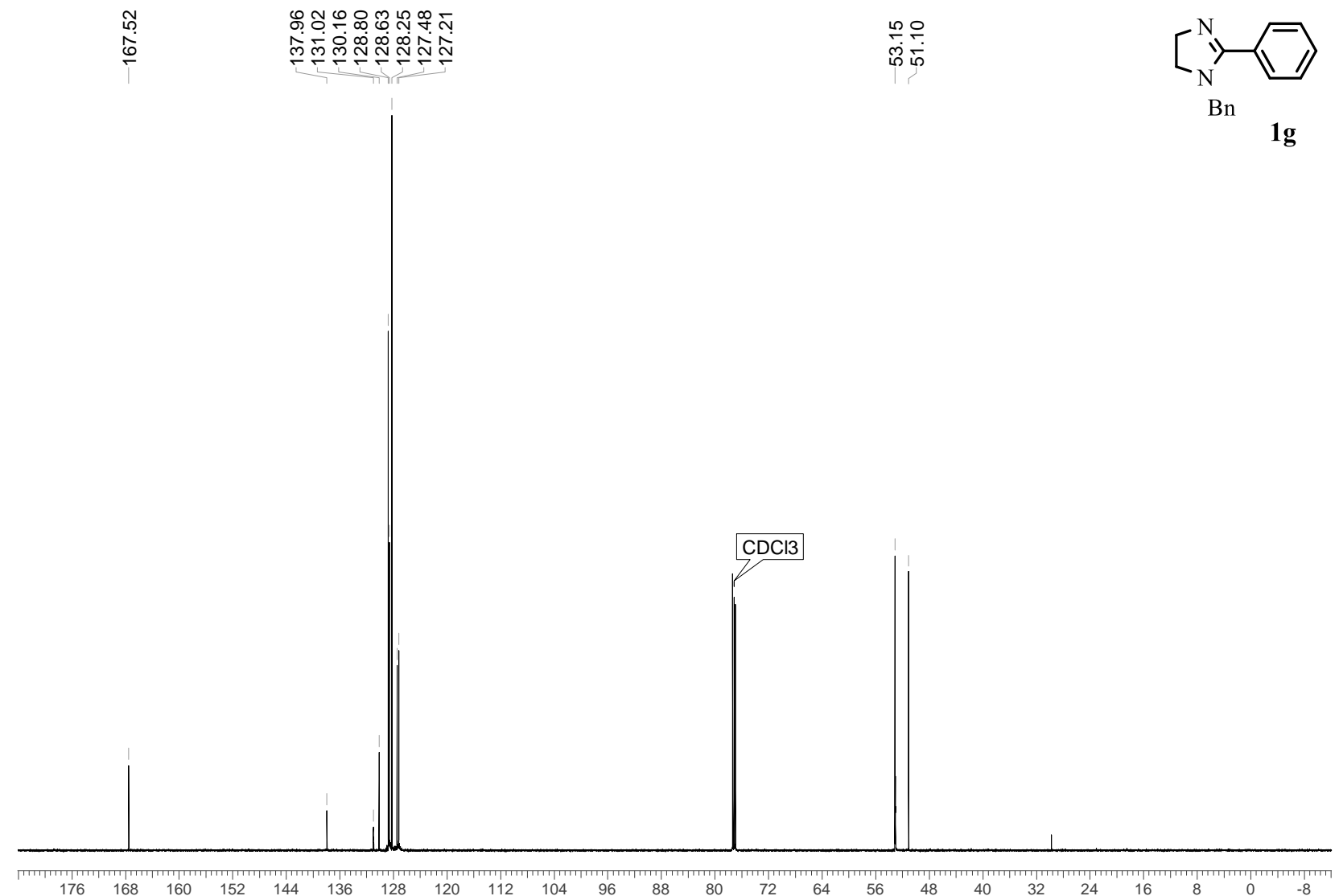


${ }^{1} \mathrm{H}$ NMR spectrum, $\mathrm{CDCl}_{3}, 600 \mathrm{MHZ}$

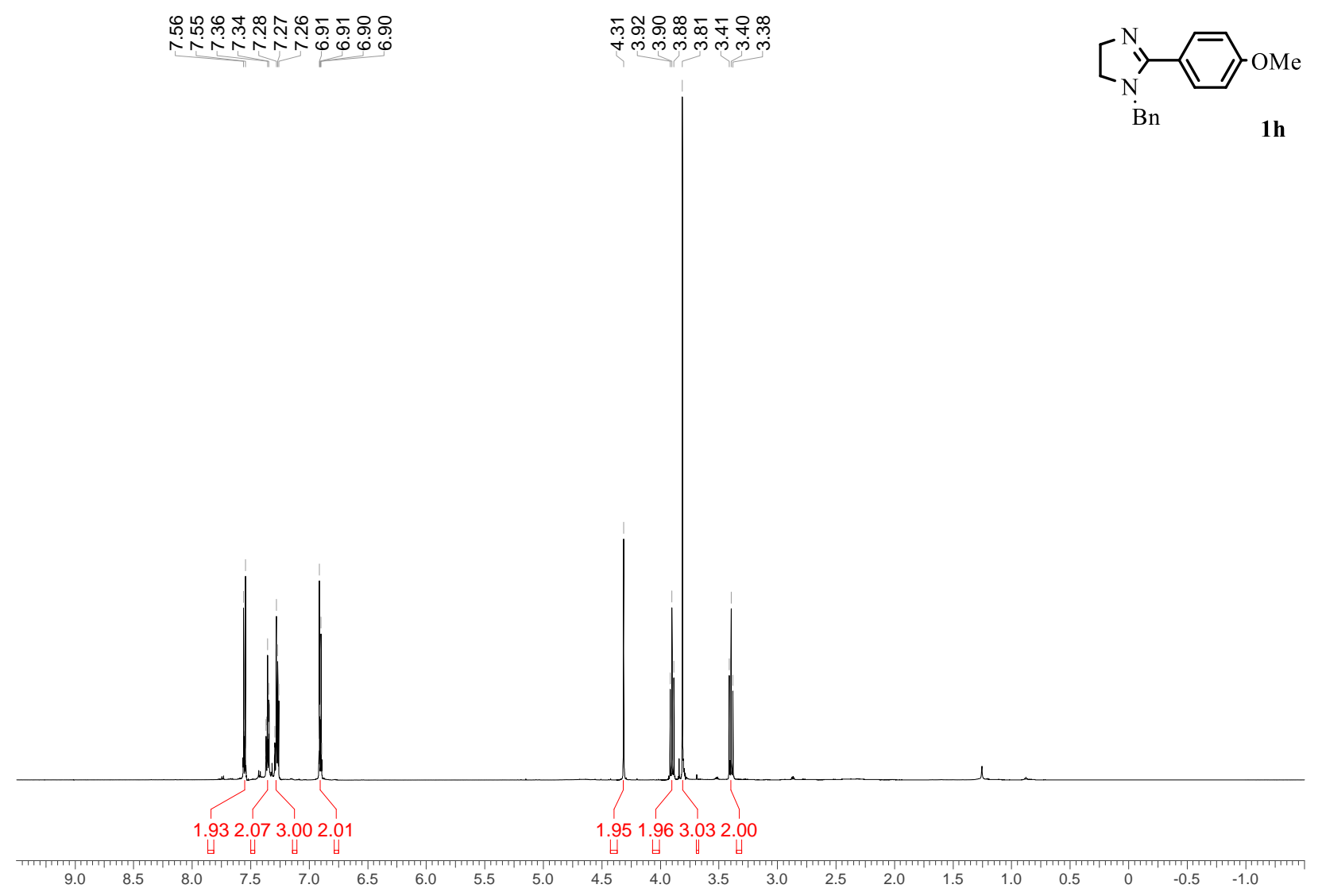

${ }^{13} \mathrm{C}$ NMR spectrum, $\mathrm{CDCl}_{3}, 150 \mathrm{MHz}$

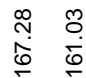

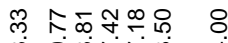

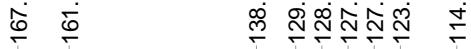

ㄱํㅇำ ํㅠำ

นึ่
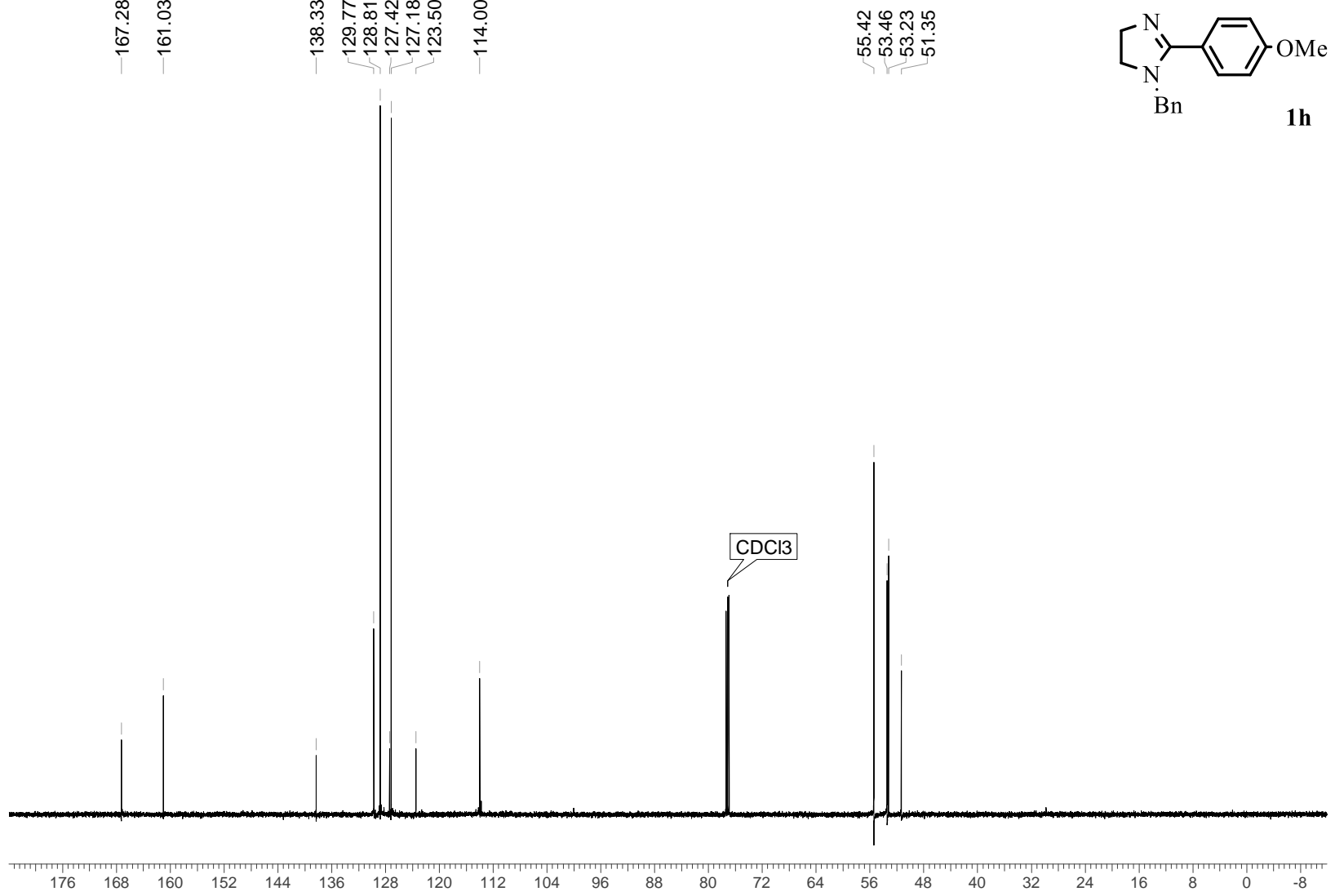
${ }^{1} \mathrm{H}$ NMR spectrum, $\mathrm{CDCl}_{3}, 600 \mathrm{MHZ}$

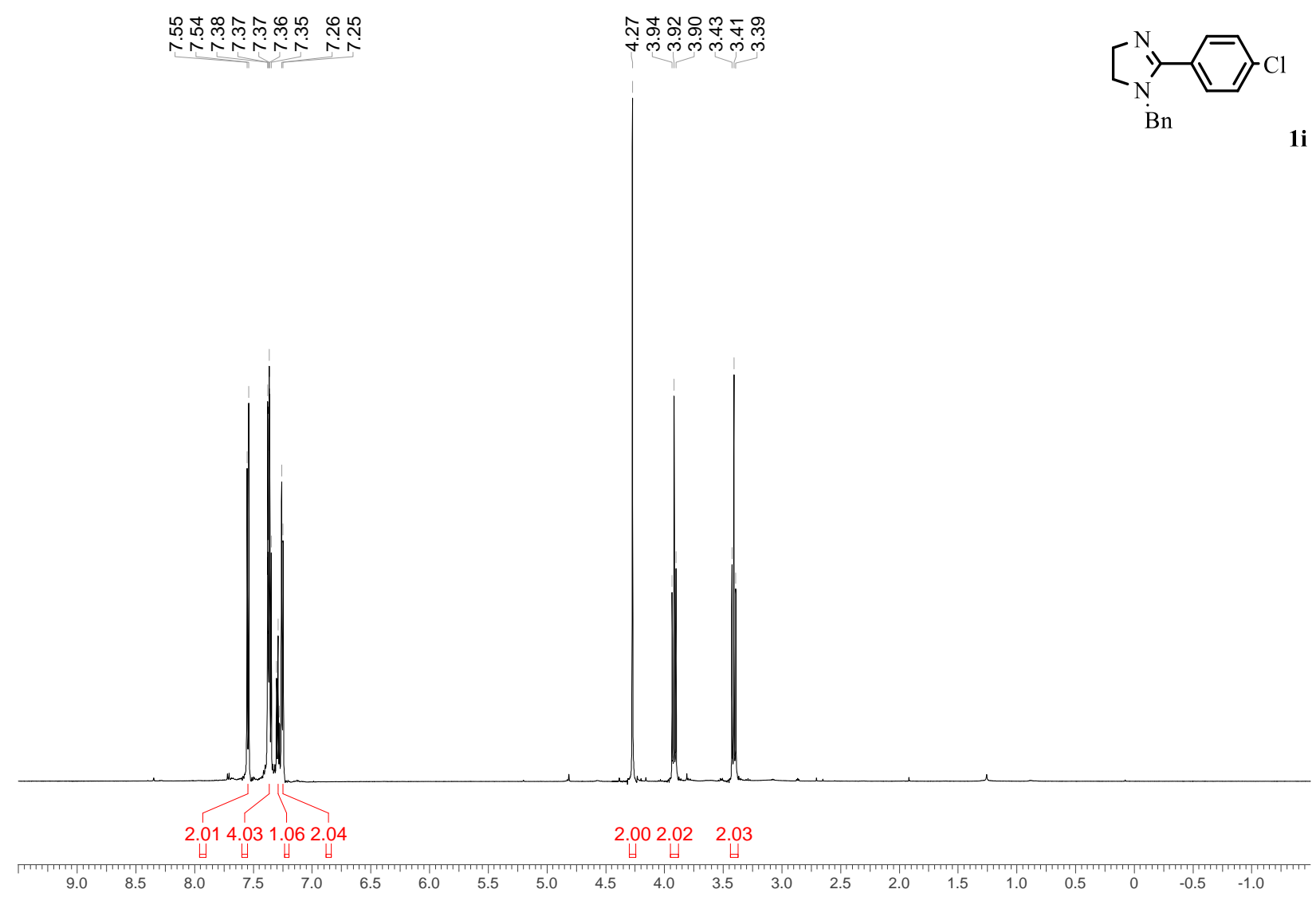

${ }^{13} \mathrm{C}$ NMR spectrum, $\mathrm{CDCl}_{3}, 150 \mathrm{MHz}$

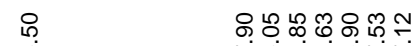

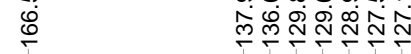

นึำกำ

ถึ่
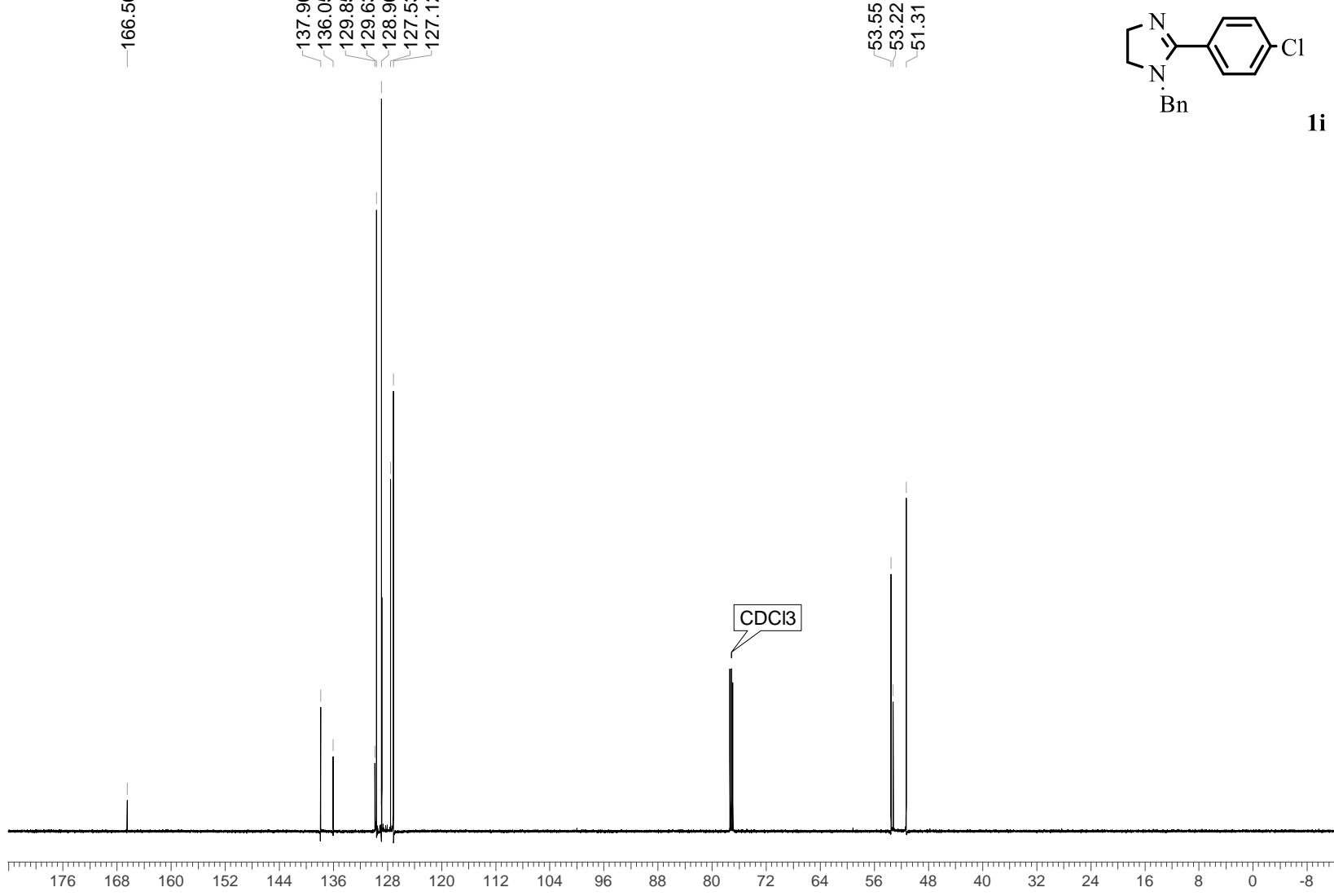
${ }^{1} \mathrm{H}$ NMR spectrum, $\mathrm{CDCl}_{3}, 600 \mathrm{MHZ}$

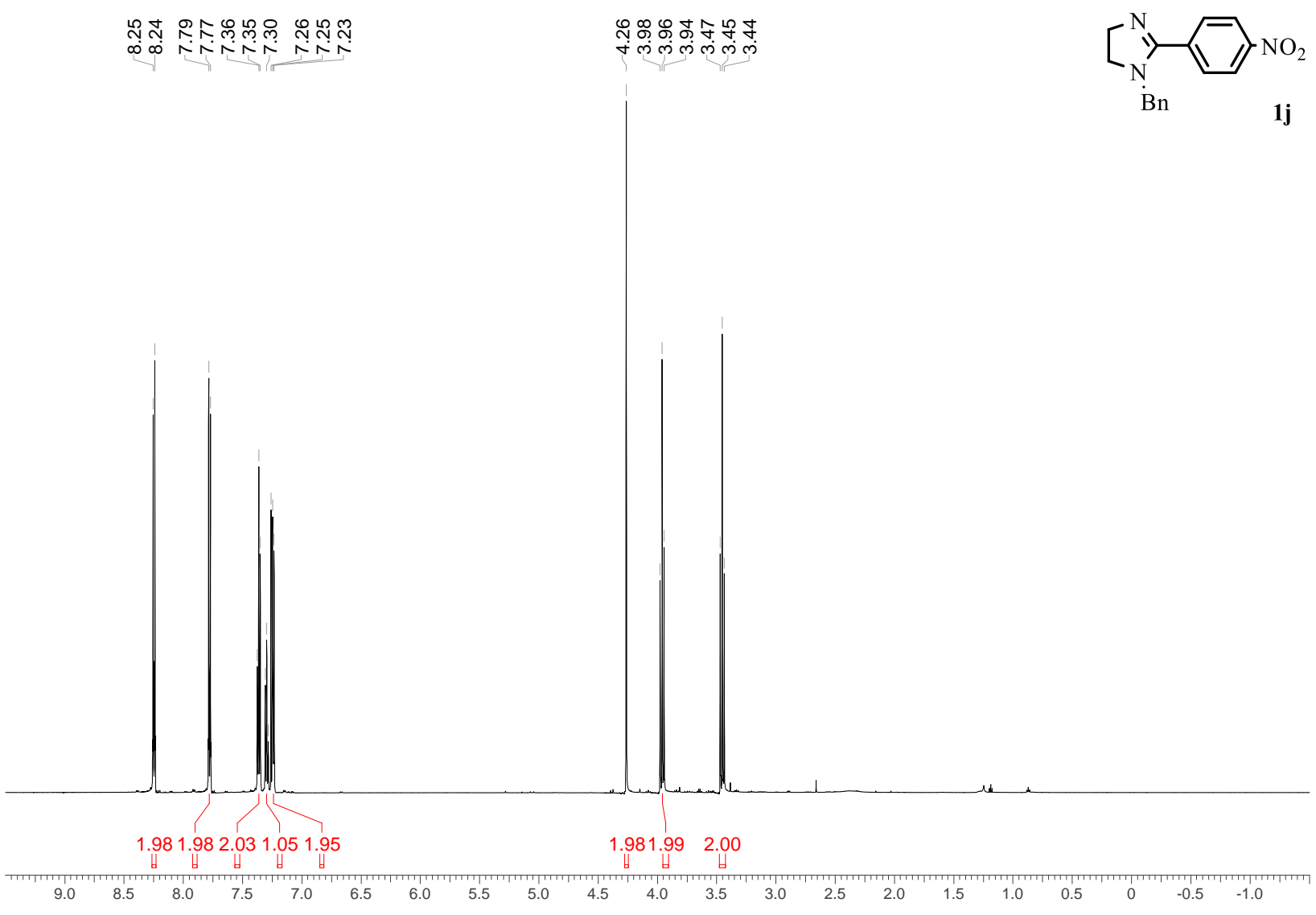

${ }^{13} \mathrm{C}$ NMR spectrum, $\mathrm{CDCl}_{3}, 150 \mathrm{MHz}$

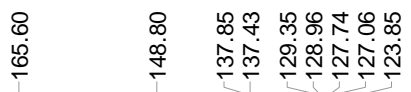

ถฯ

ณึ่ ถุำ
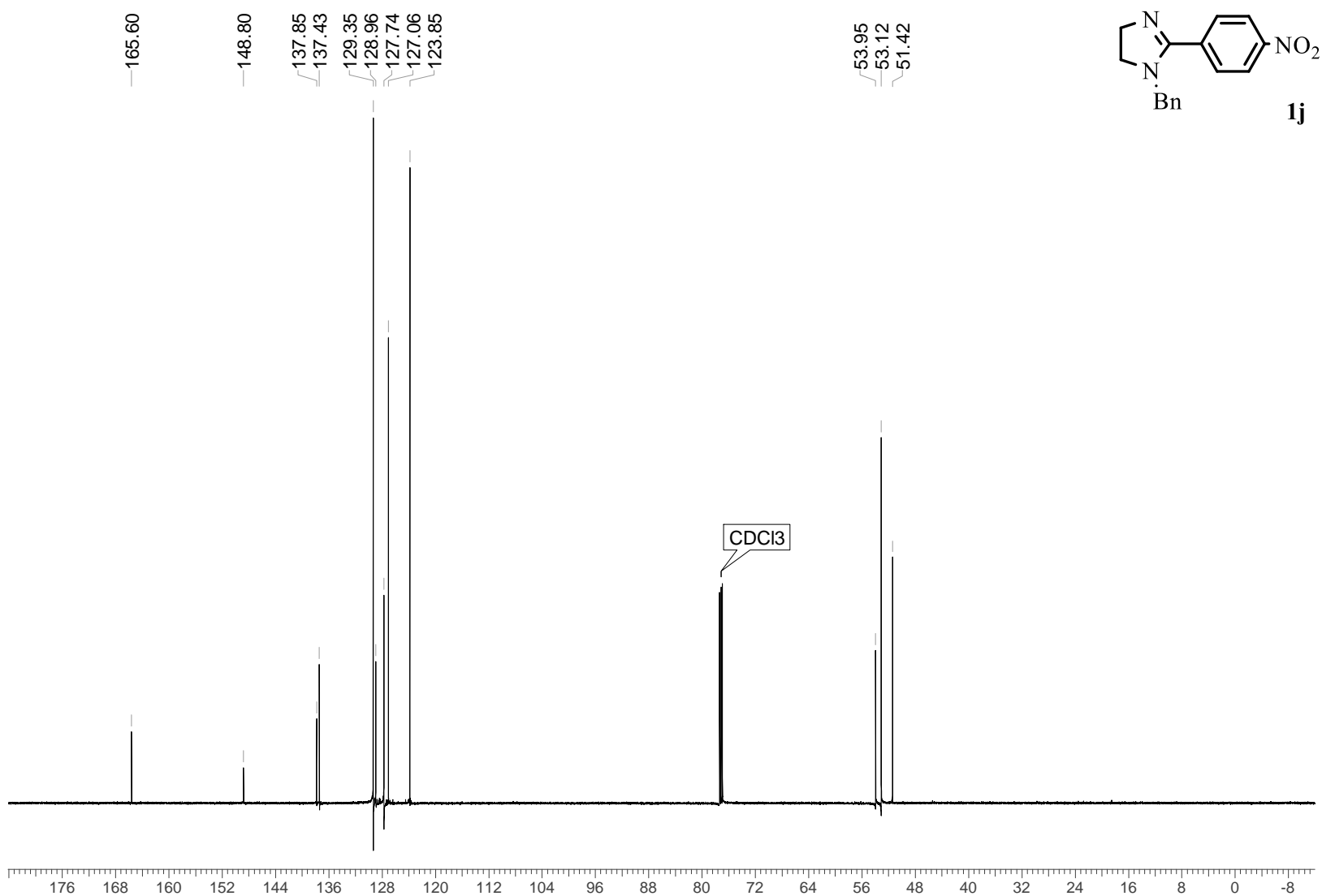
${ }^{1} \mathrm{H}$ NMR spectrum, $\mathrm{CDCl}_{3}, 600 \mathrm{MHZ}$

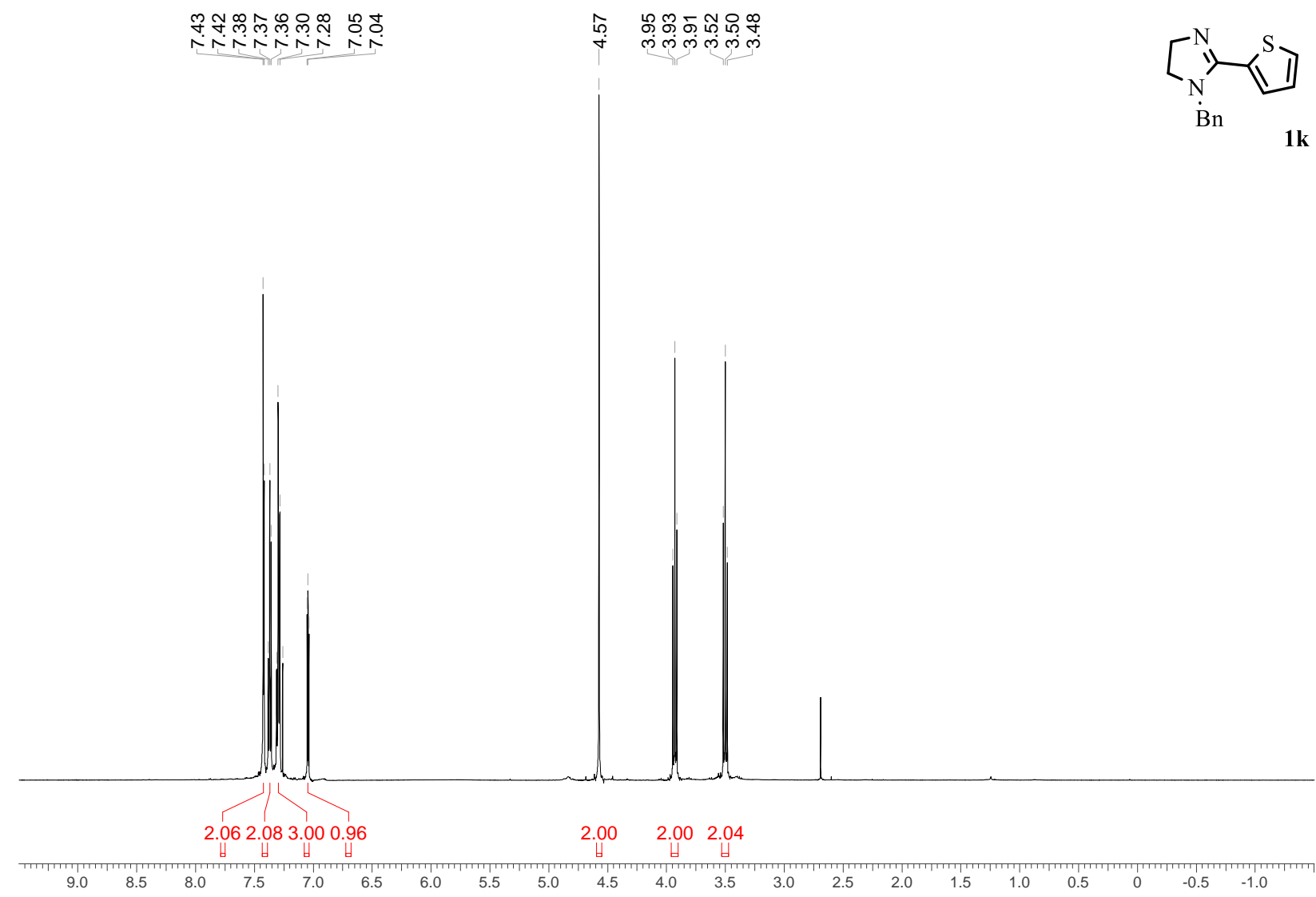

${ }^{13} \mathrm{C}$ NMR spectrum, $\mathrm{CDCl}_{3}, 150 \mathrm{MHz}$

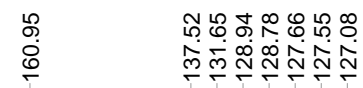

\&̊ㅗ.

กิ่

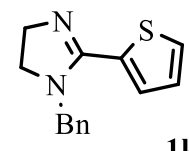

$1 \mathrm{k}$

$1768_{168}$ 
${ }^{1} \mathrm{H}$ NMR spectrum, $\mathrm{CDCl}_{3}, 600 \mathrm{MHZ}$

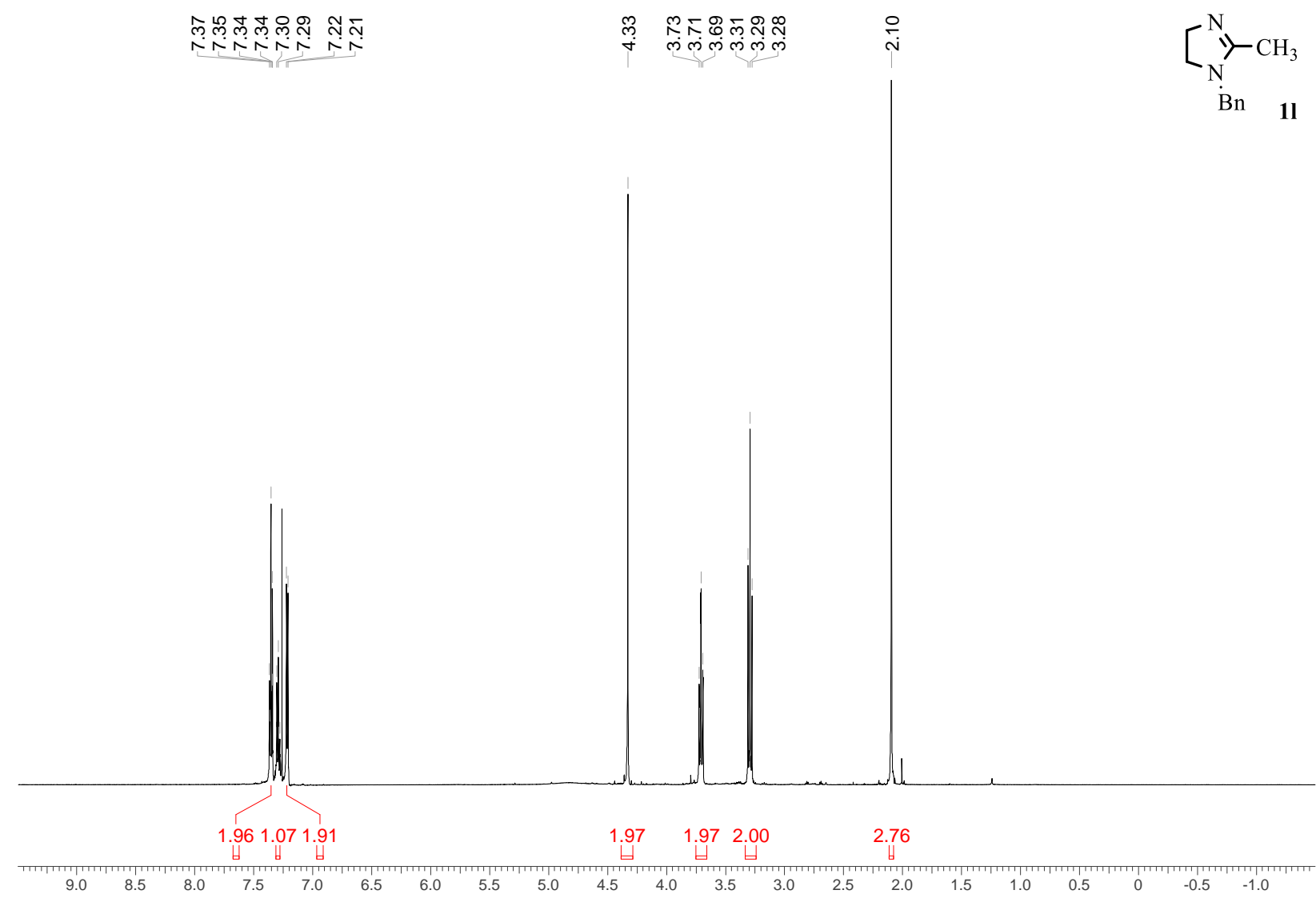

${ }^{13} \mathrm{C}$ NMR spectrum, $\mathrm{CDCl}_{3}, 150 \mathrm{MHz}$

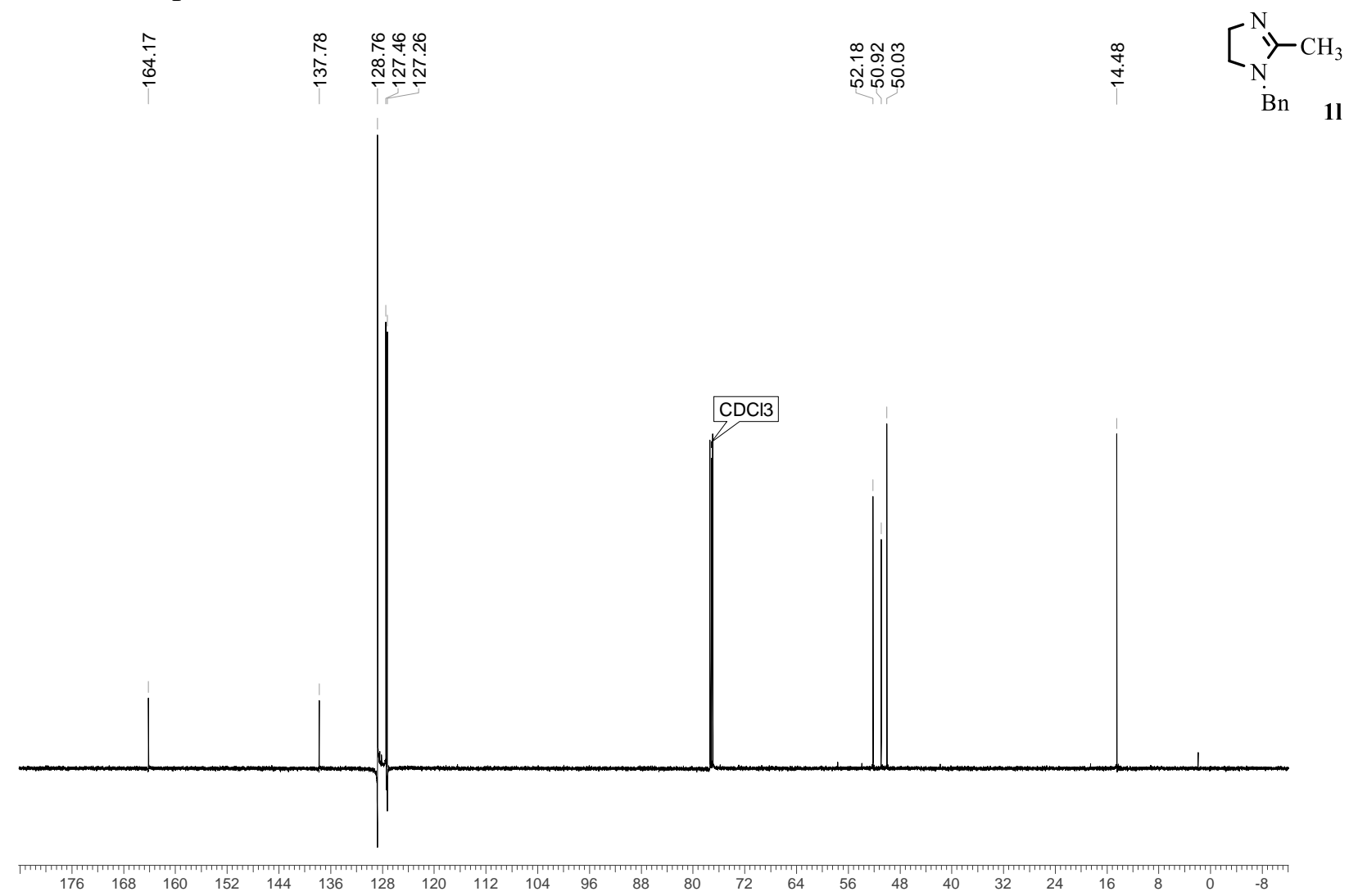


${ }^{1} \mathrm{H}$ NMR spectrum, $\mathrm{CDCl}_{3}, 600 \mathrm{MHZ}$

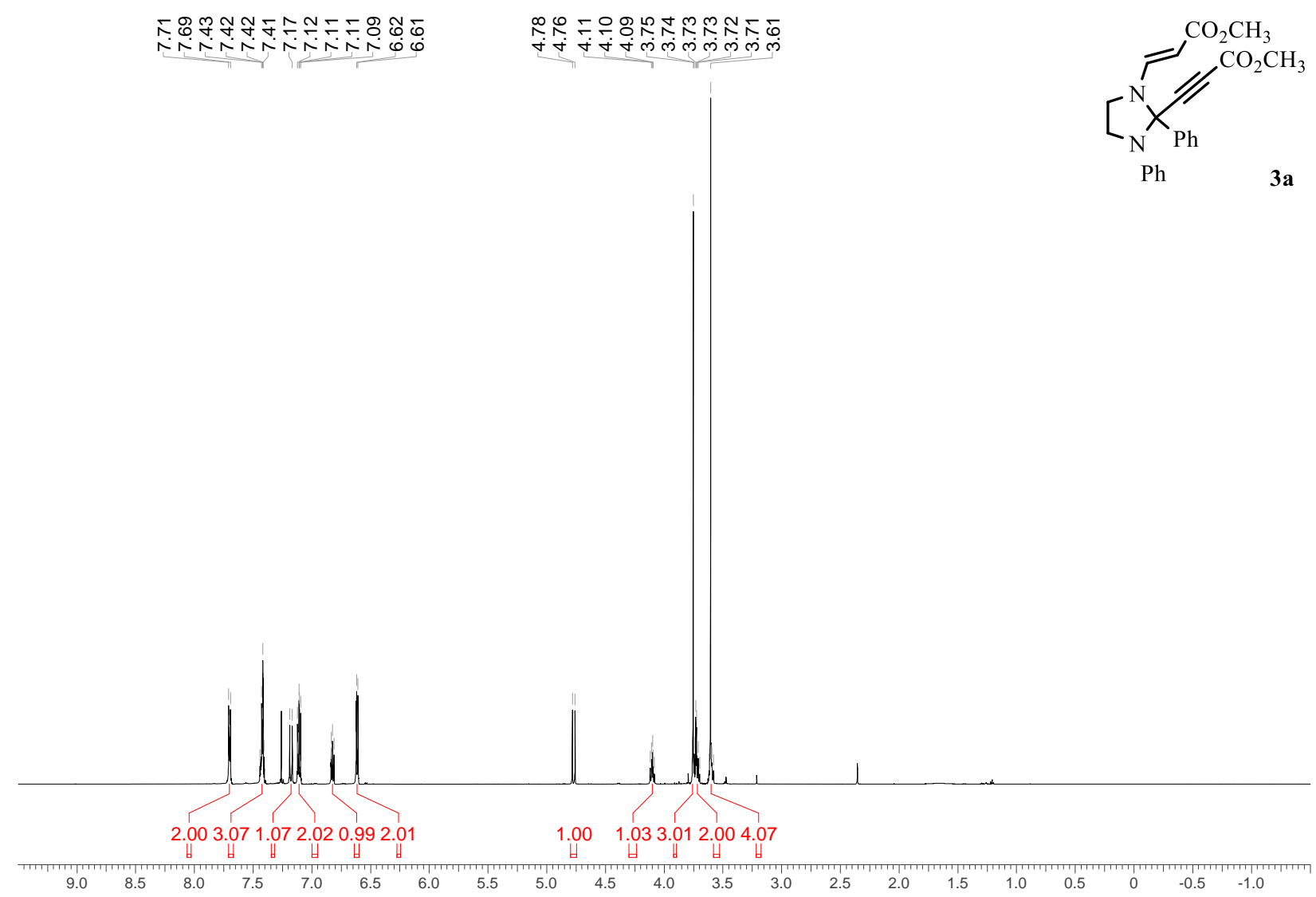

${ }^{13} \mathrm{C}$ NMR spectrum, $\mathrm{CDCl}_{3}, 150 \mathrm{MHz}$

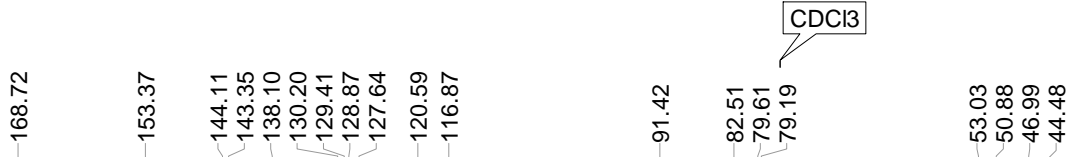
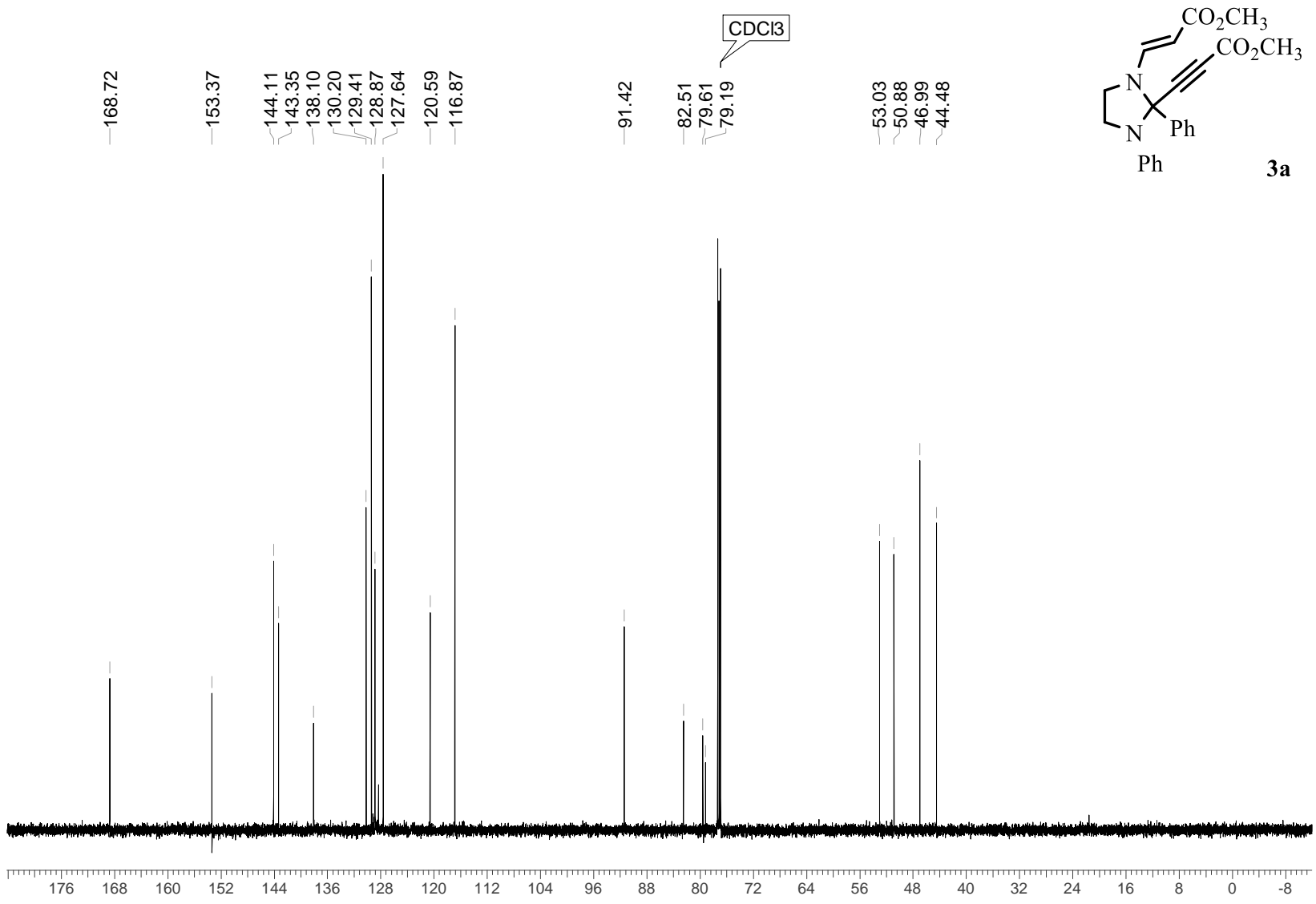
${ }^{1} \mathrm{H}$ NMR spectrum, $\mathrm{CDCl}_{3}, 600 \mathrm{MHZ}$

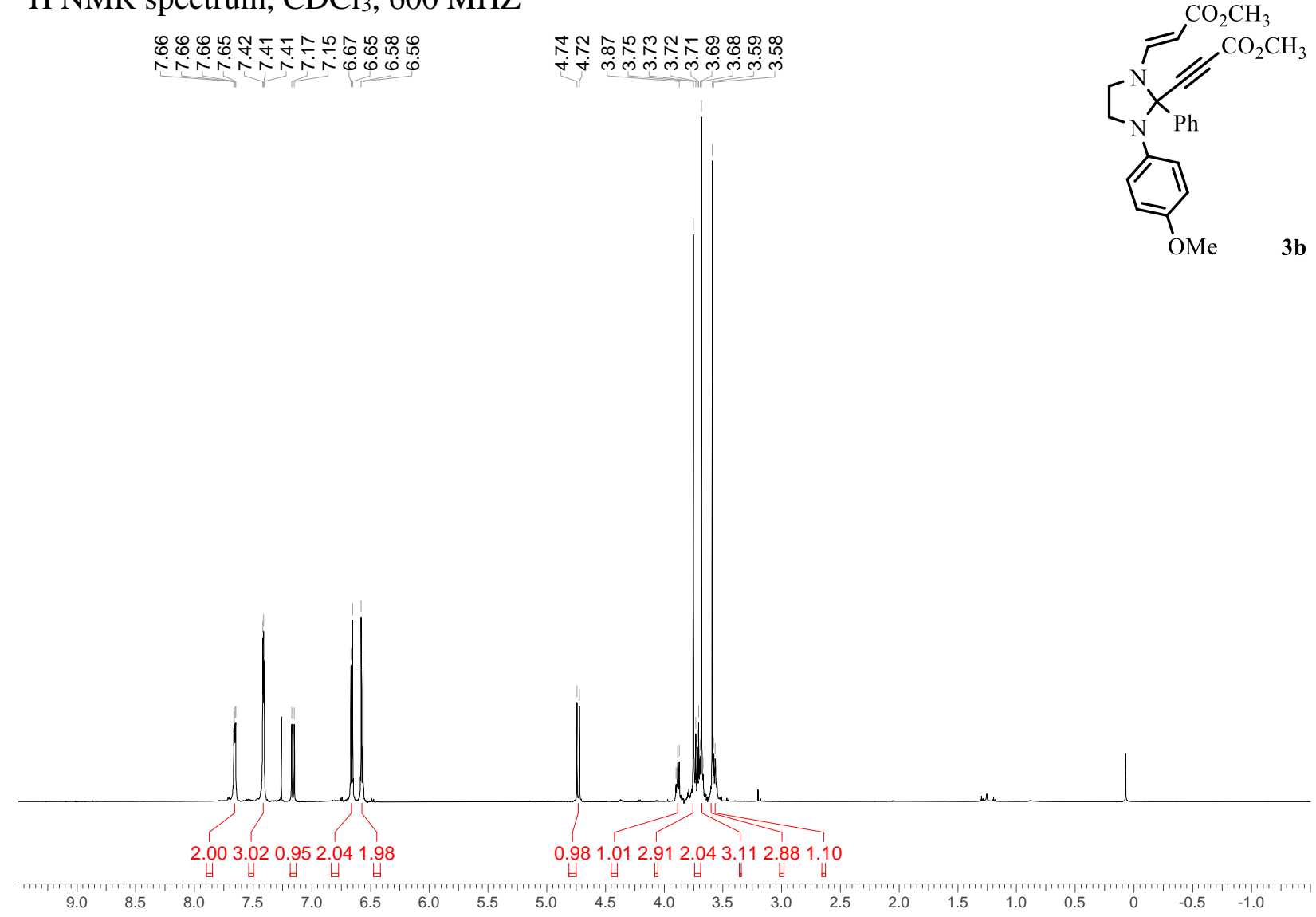

${ }^{13} \mathrm{C}$ NMR spectrum, $\mathrm{CDCl}_{3}, 150 \mathrm{MHz}$

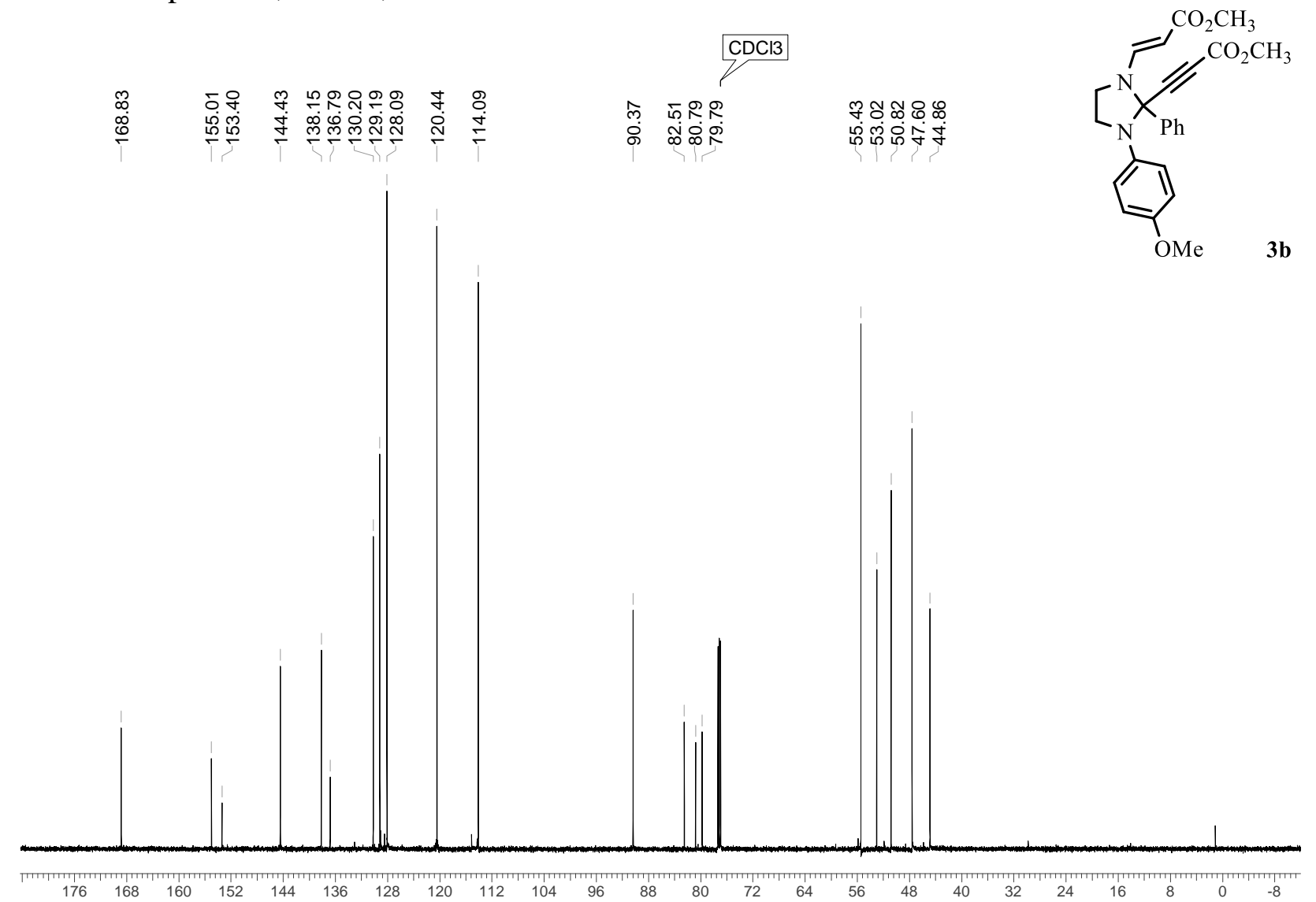


${ }^{1} \mathrm{H}$ NMR spectrum, $\mathrm{CDCl}_{3}, 600 \mathrm{MHZ}$

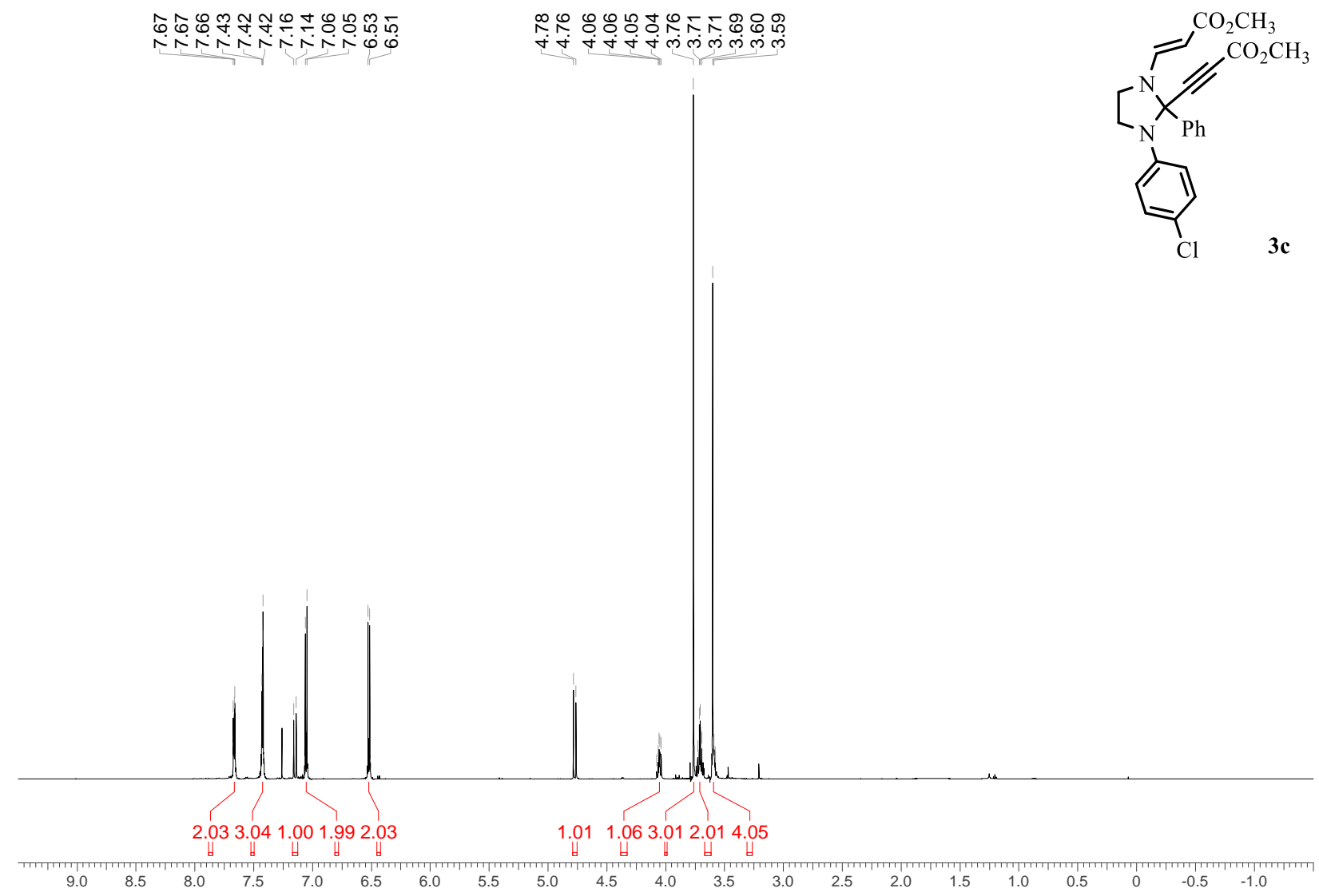

${ }^{13} \mathrm{C}$ NMR spectrum, $\mathrm{CDCl}_{3}, 150 \mathrm{MHz}$

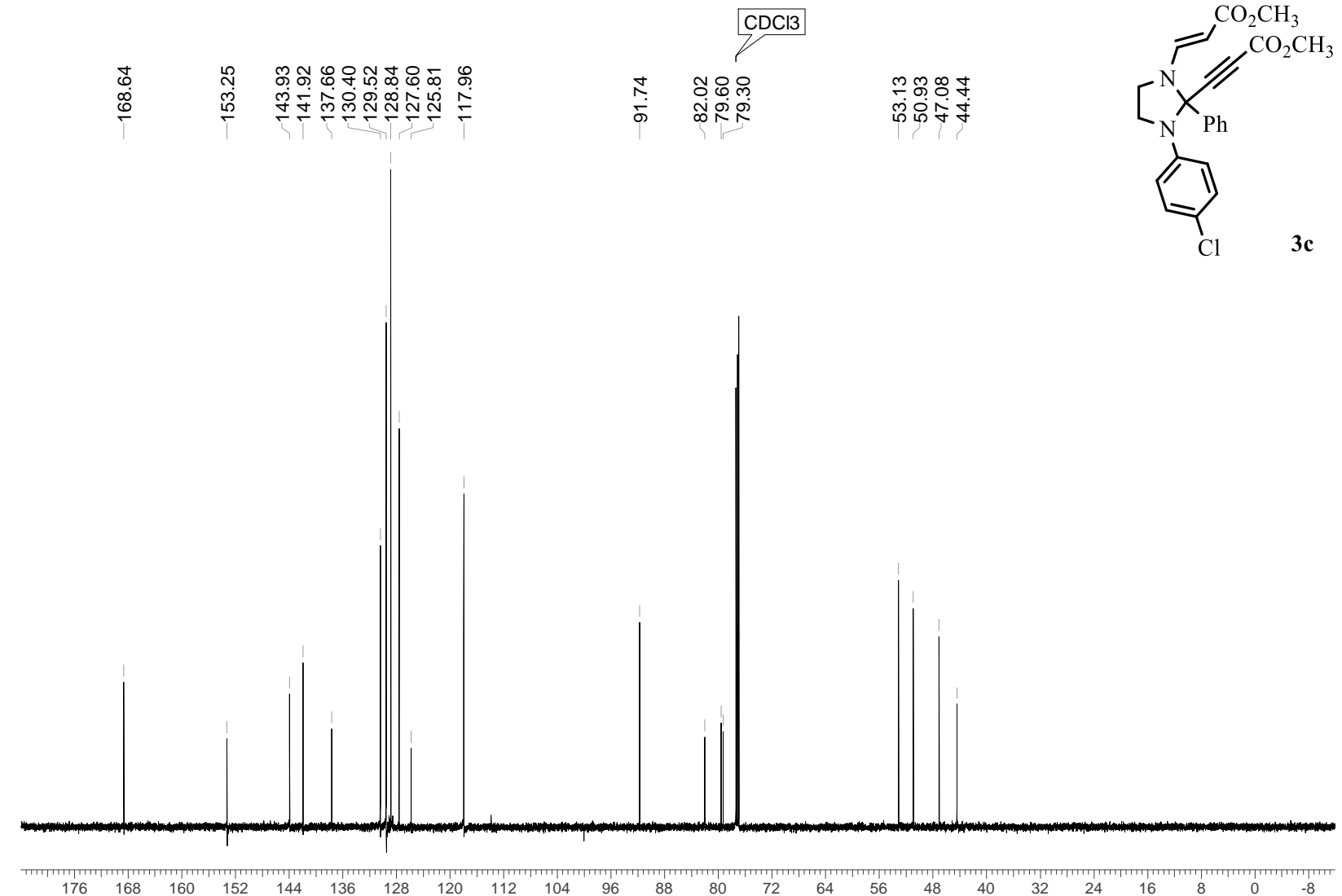


${ }^{1} \mathrm{H}$ NMR spectrum, $\mathrm{CDCl}_{3}, 600 \mathrm{MHZ}$

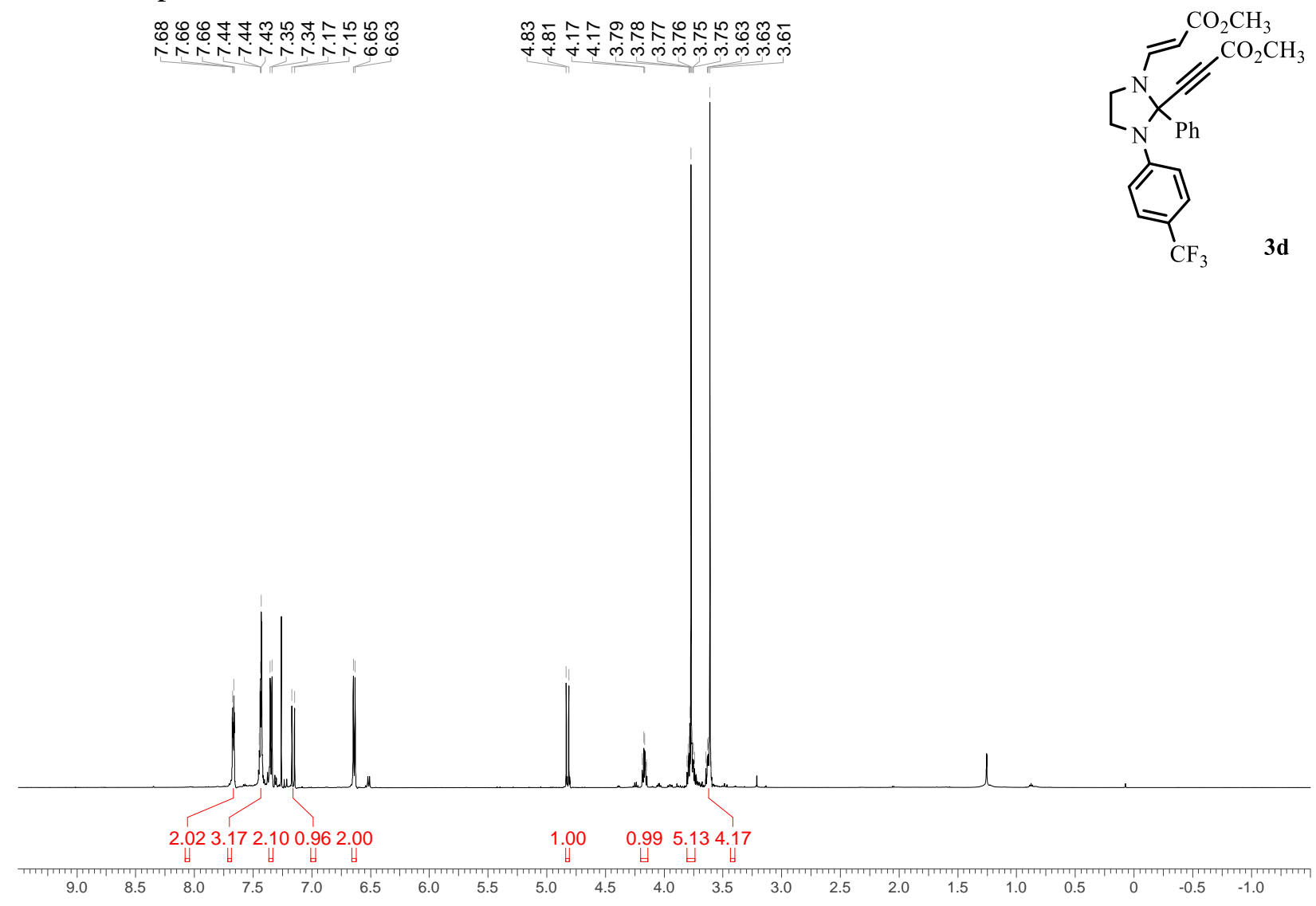

${ }^{13} \mathrm{C}$ NMR spectrum, $\mathrm{CDCl}_{3}, 150 \mathrm{MHz}$

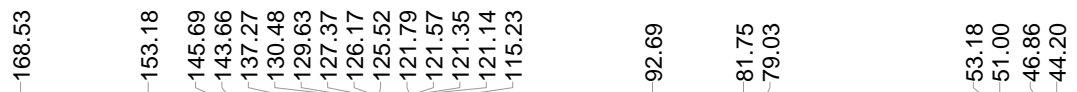

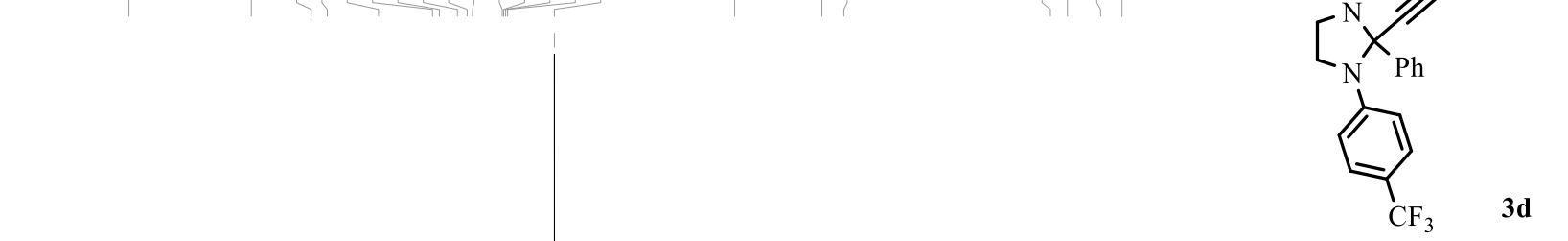


${ }^{1} \mathrm{H}$ NMR spectrum, $\mathrm{CDCl}_{3}, 600 \mathrm{MHZ}$

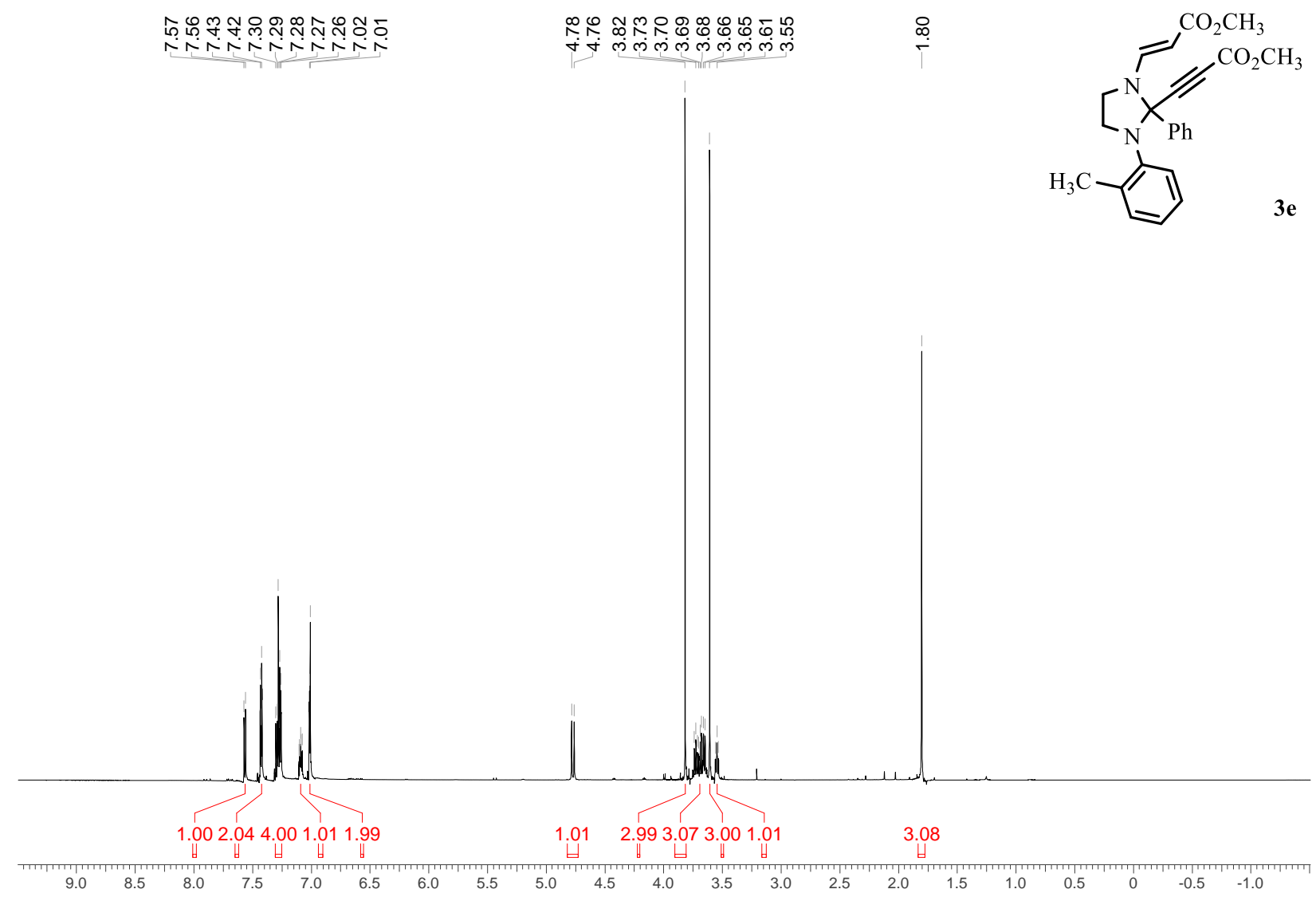

${ }^{13} \mathrm{C}$ NMR spectrum, $\mathrm{CDCl}_{3}, 150 \mathrm{MHz}$

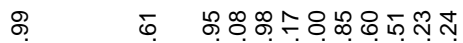

至

के హु

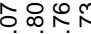

凩绍守尔

$\stackrel{\infty}{\stackrel{\circ}{\Gamma}}$

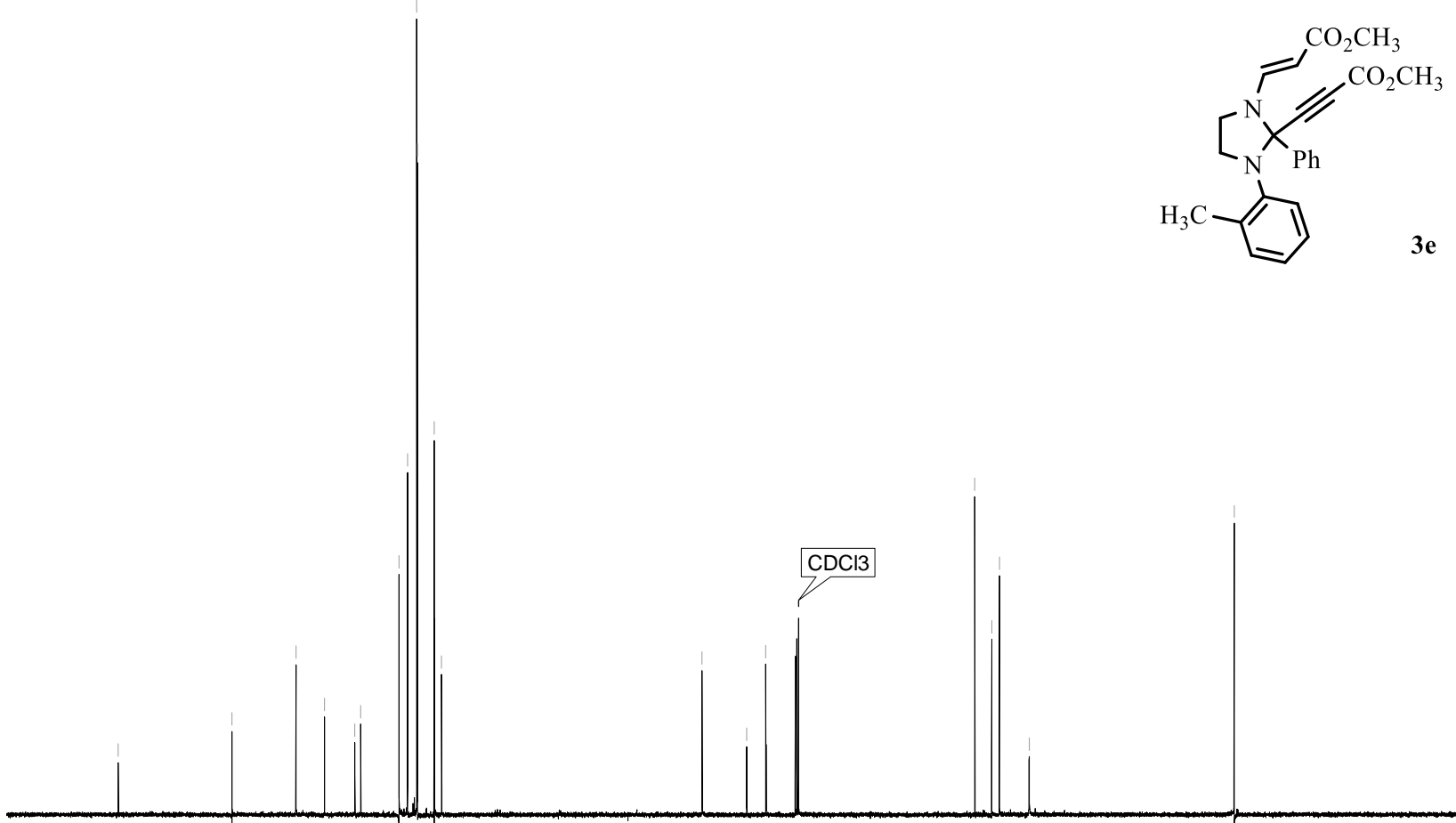

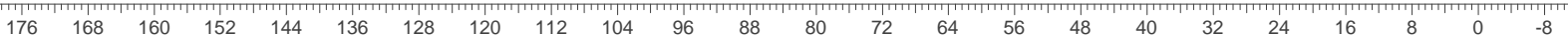


${ }^{1} \mathrm{H}$ NMR spectrum, $\mathrm{CDCl}_{3}, 600 \mathrm{MHZ}$

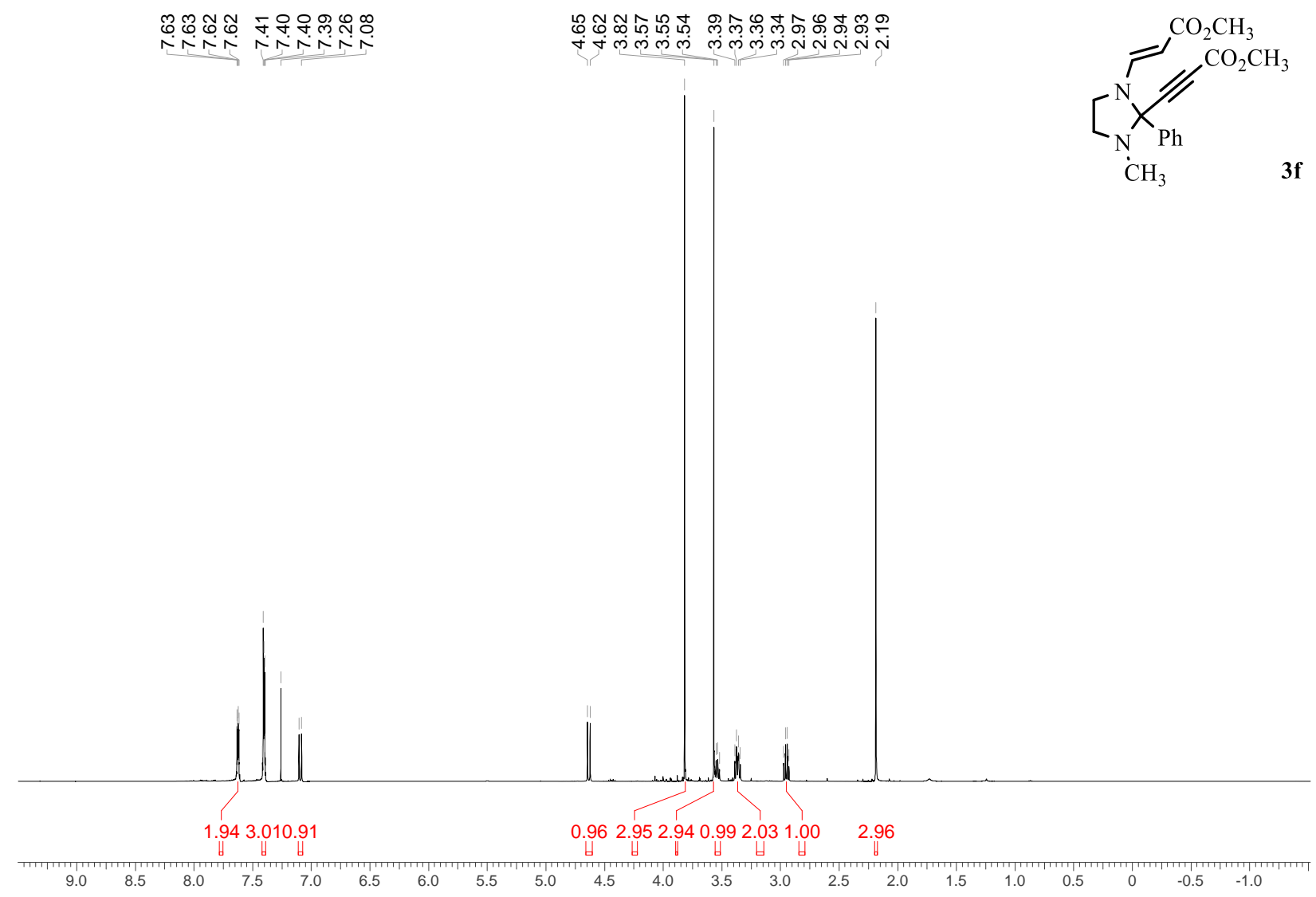

${ }^{13} \mathrm{C}$ NMR spectrum, $\mathrm{CDCl}_{3}, 150 \mathrm{MHz}$

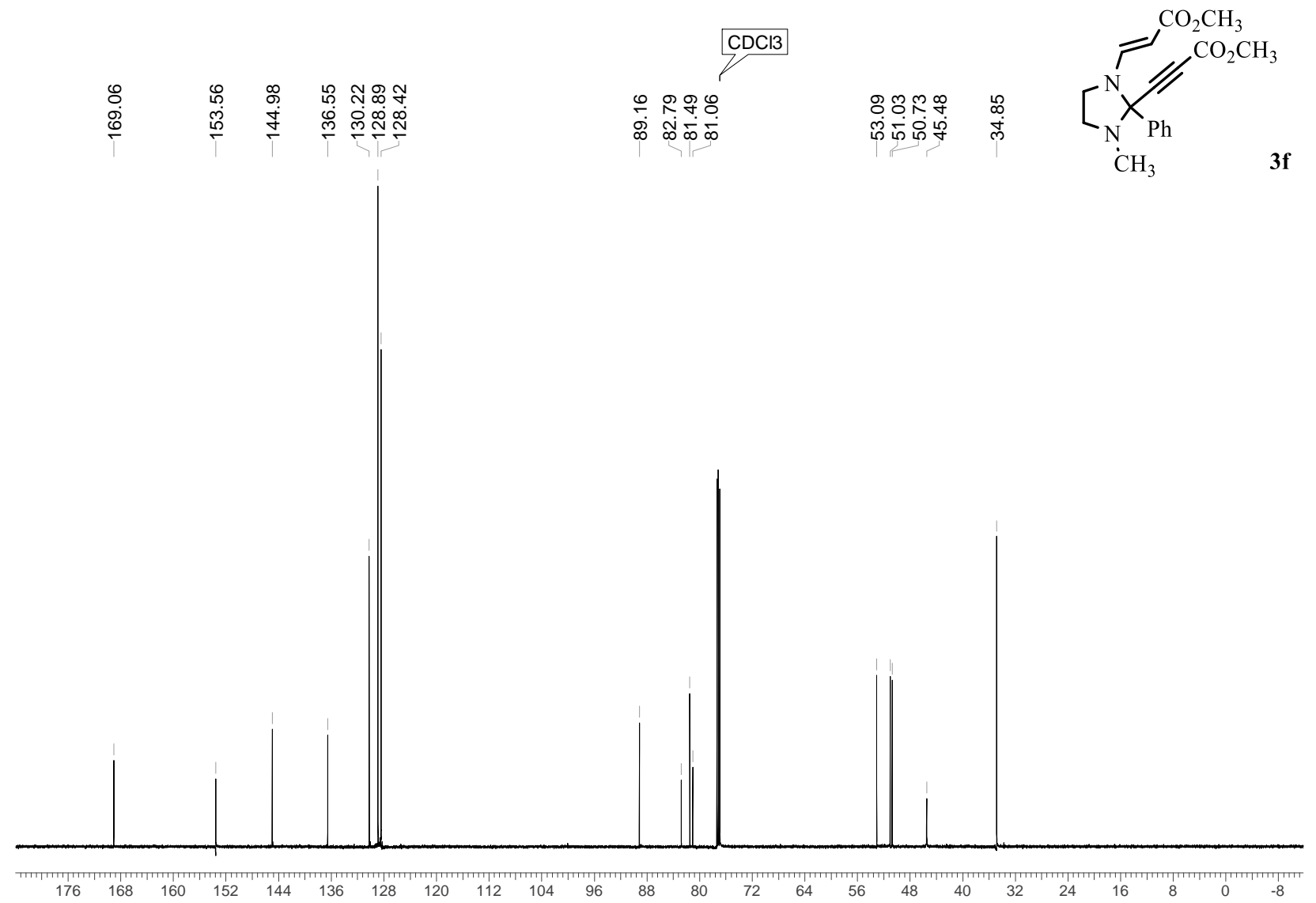


${ }^{1} \mathrm{H}$ NMR spectrum, $\mathrm{CDCl}_{3}, 600 \mathrm{MHZ}$

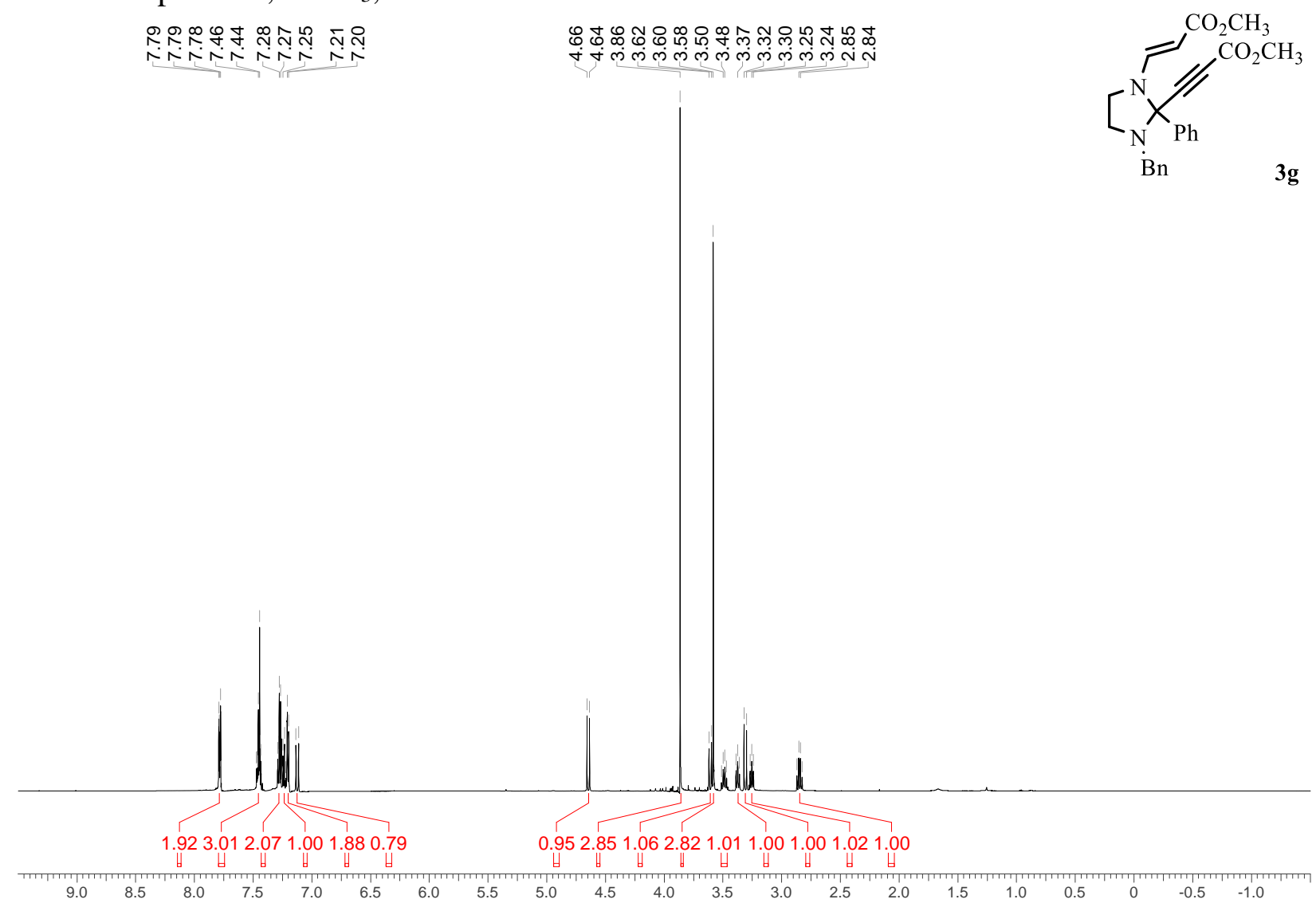

${ }^{13} \mathrm{C}$ NMR spectrum, $\mathrm{CDCl}_{3}, 150 \mathrm{MHz}$
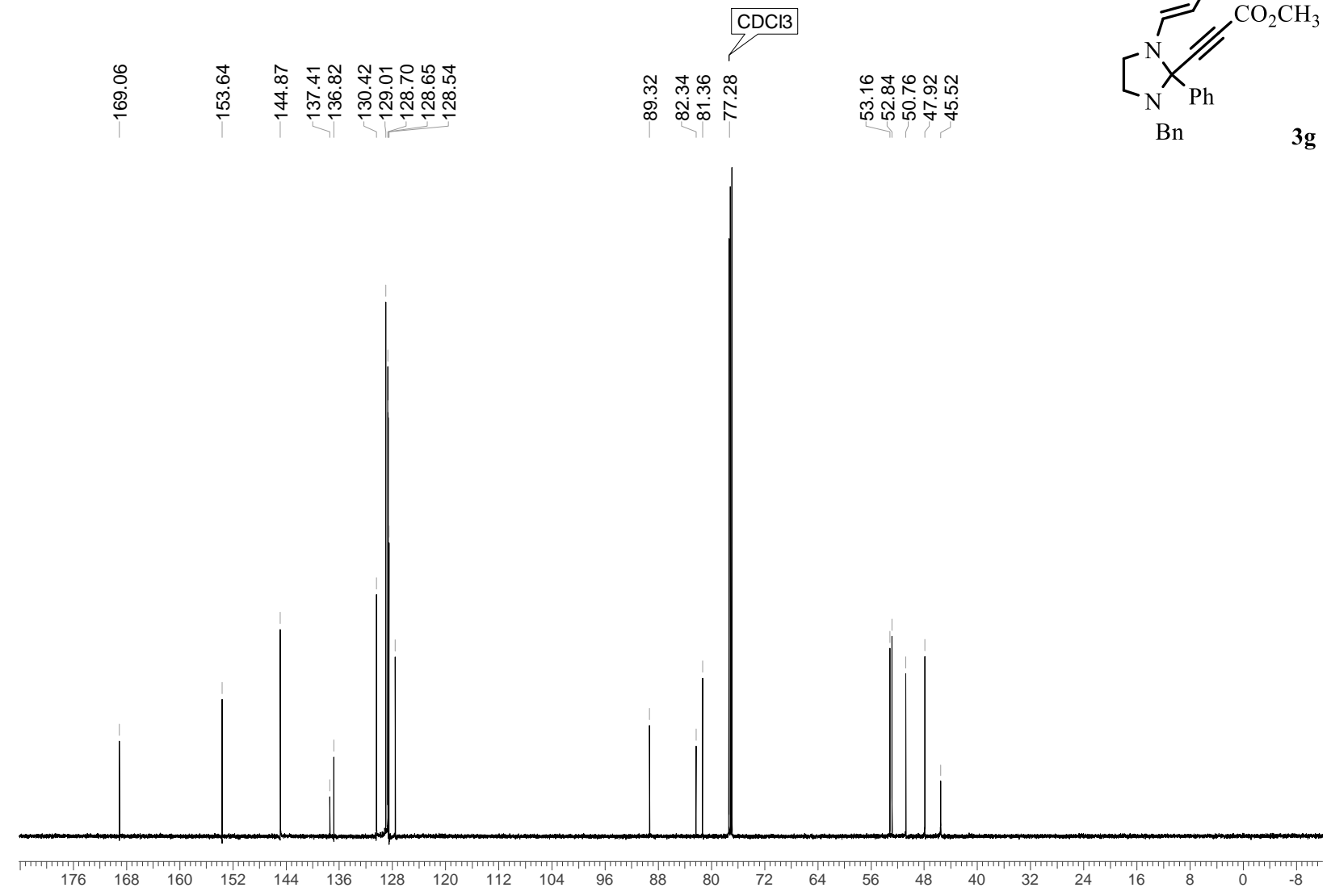
${ }^{1} \mathrm{H}$ NMR spectrum, $\mathrm{CDCl}_{3}, 600 \mathrm{MHZ}$

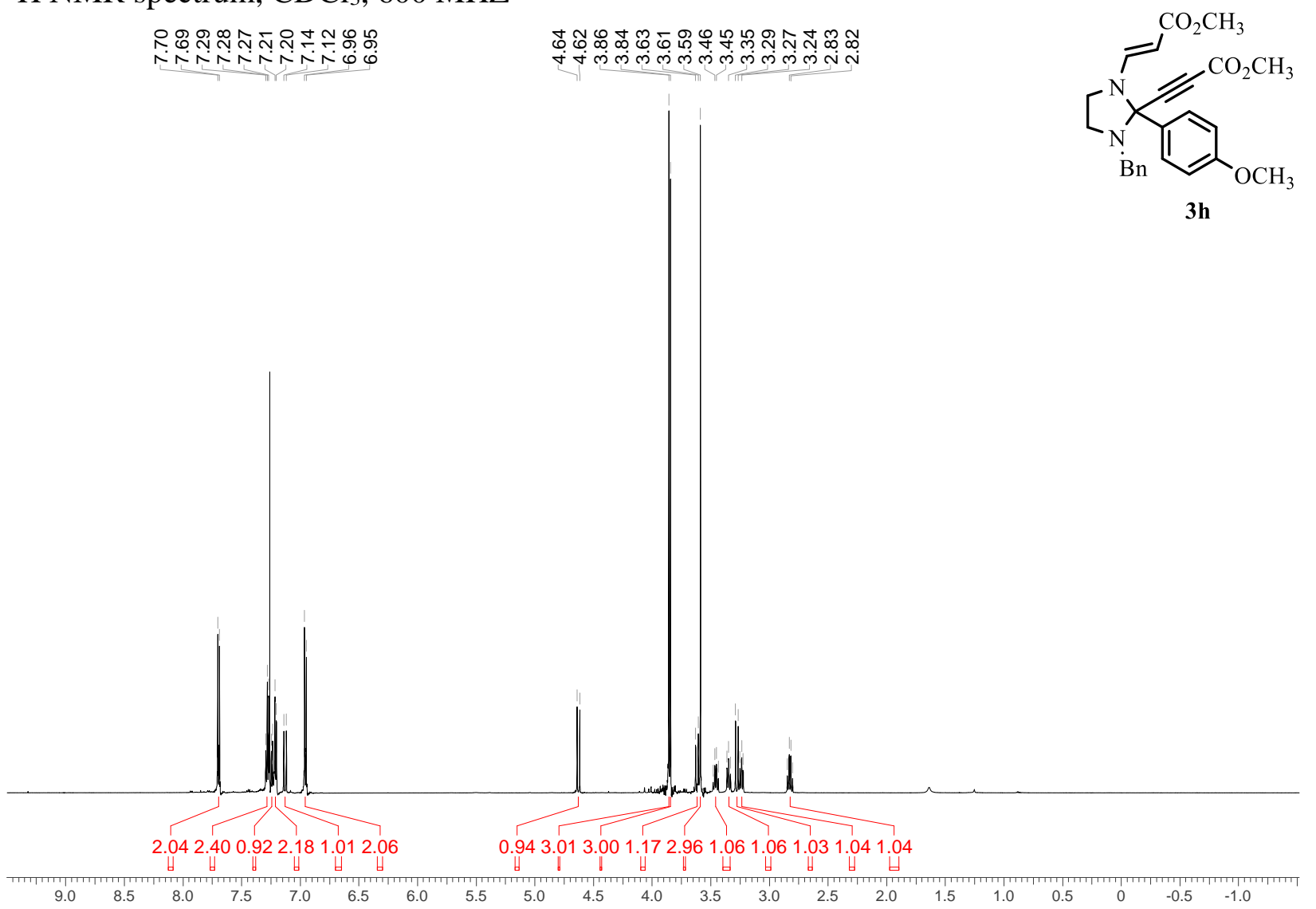

${ }^{13} \mathrm{C}$ NMR spectrum, $\mathrm{CDCl}_{3}, 150 \mathrm{MHz}$

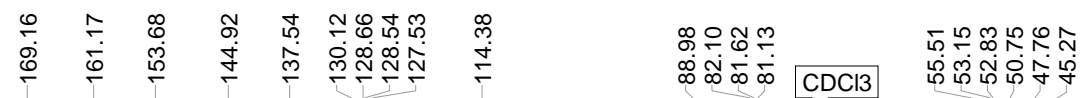

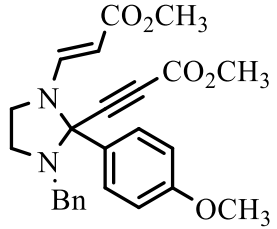

3h 
${ }^{1} \mathrm{H}$ NMR spectrum, $\mathrm{CDCl}_{3}, 600 \mathrm{MHZ}$

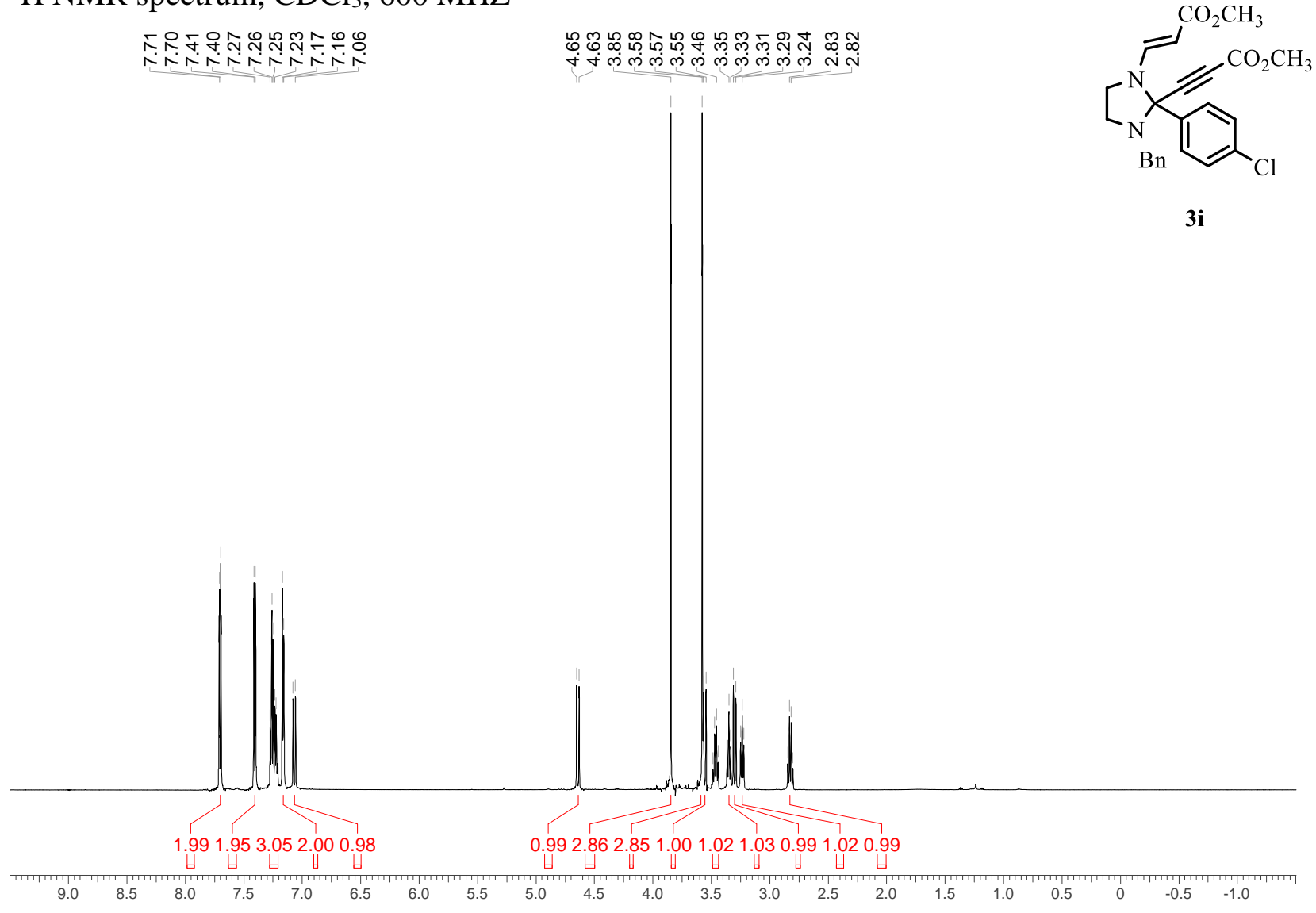

${ }^{13} \mathrm{C}$ NMR spectrum, $\mathrm{CDCl}_{3}, 150 \mathrm{MHz}$

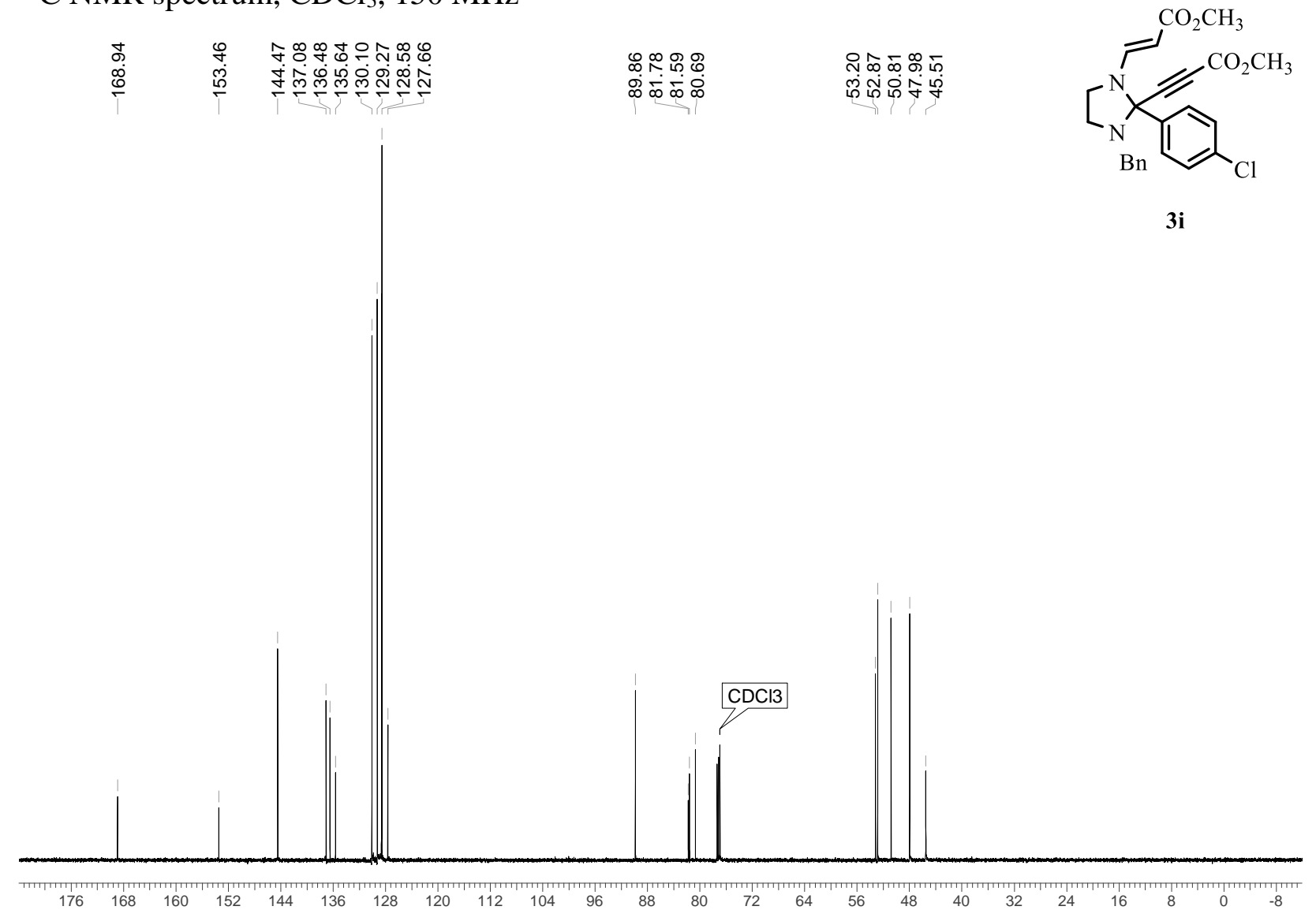


${ }^{1} \mathrm{H}$ NMR spectrum, $\mathrm{CDCl}_{3}, 600 \mathrm{MHZ}$

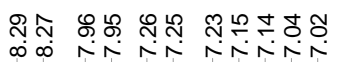

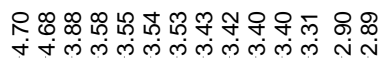

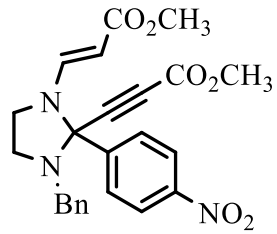

$3 \mathbf{j}$

$2.041 .96 \quad 3.221 .940 .90$

$0.98 \quad 2.982 .862 .071 .980 .970 .99$

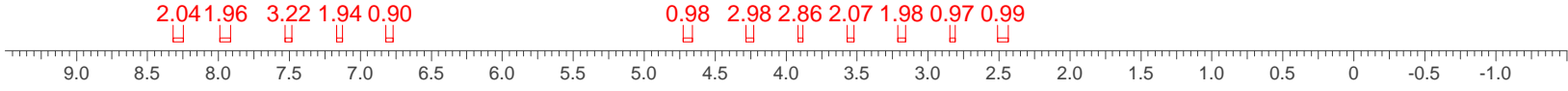

${ }^{13} \mathrm{C}$ NMR spectrum, $\mathrm{CDCl}_{3}, 150 \mathrm{MHz}$

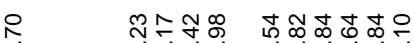

则

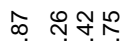

安

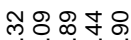

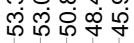

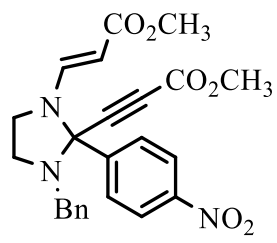

3j

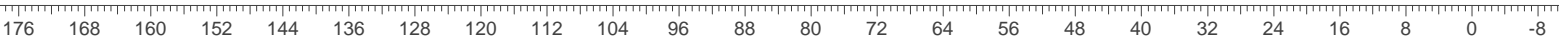


${ }^{1} \mathrm{H}$ NMR spectrum, $\mathrm{CDCl}_{3}, 600 \mathrm{MHZ}$

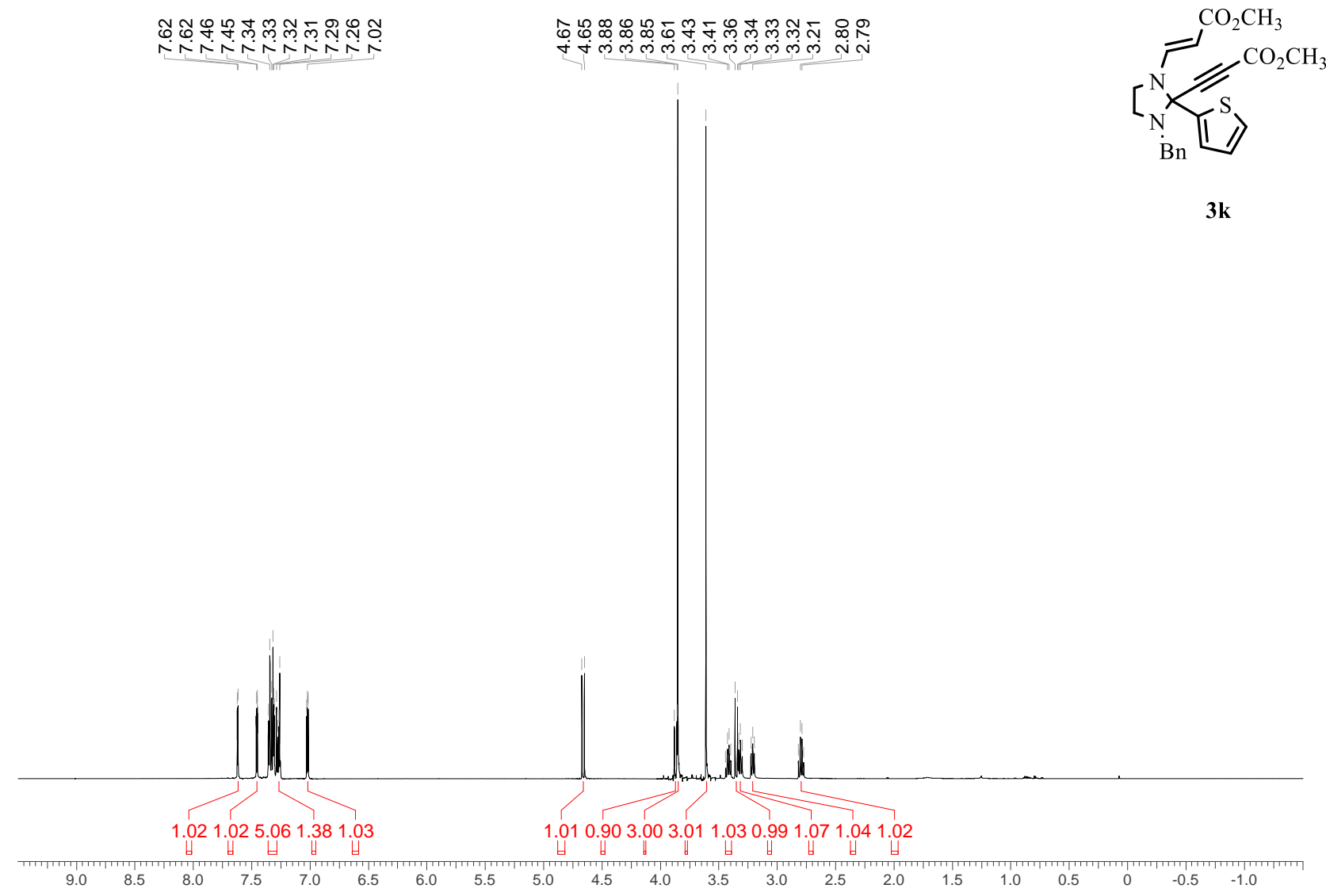

${ }^{13} \mathrm{C}$ NMR spectrum, $\mathrm{CDCl}_{3}, 150 \mathrm{MHz}$

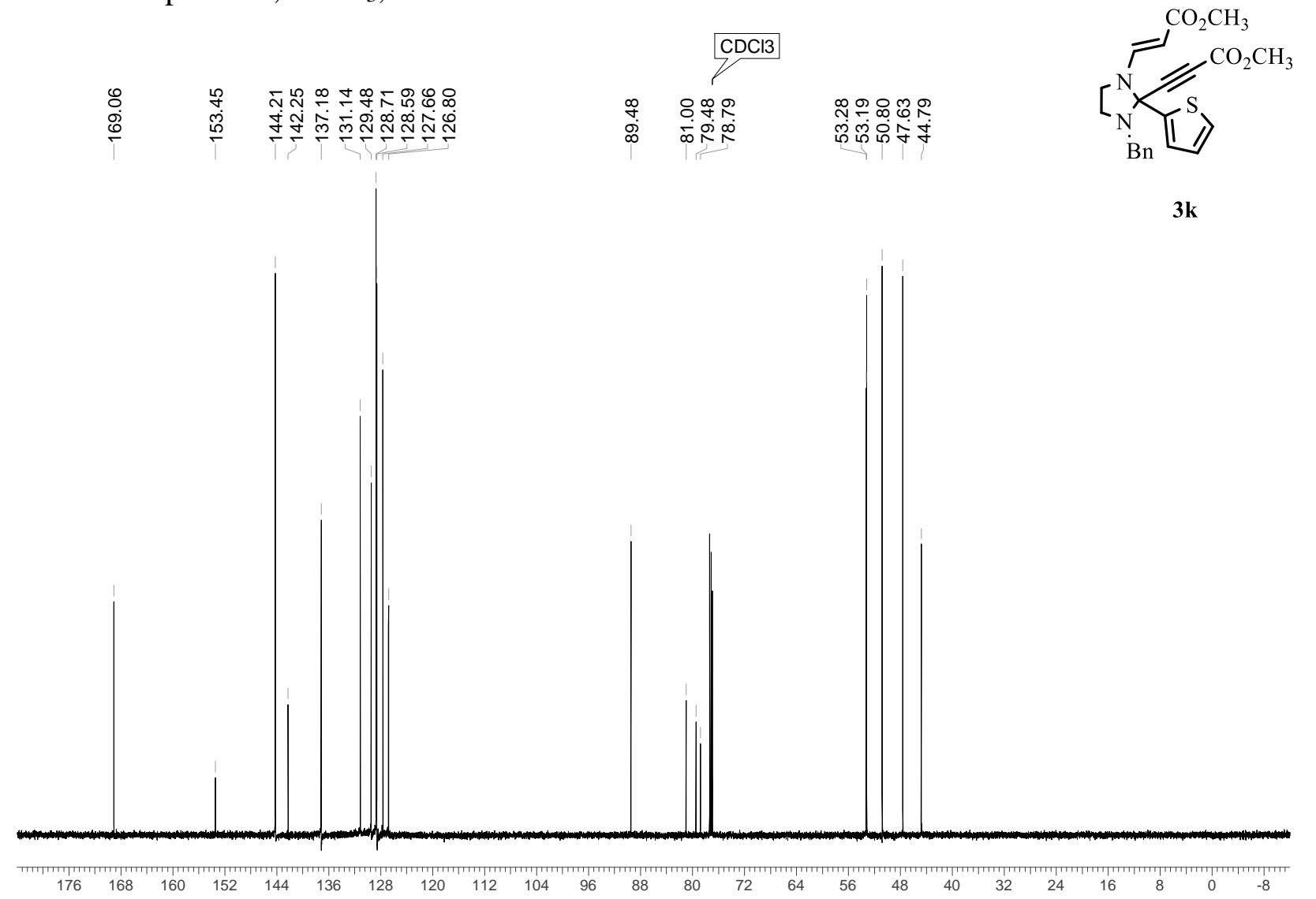


${ }^{1} \mathrm{H}$ NMR spectrum, $\mathrm{CDCl}_{3}, 600 \mathrm{MHZ}$

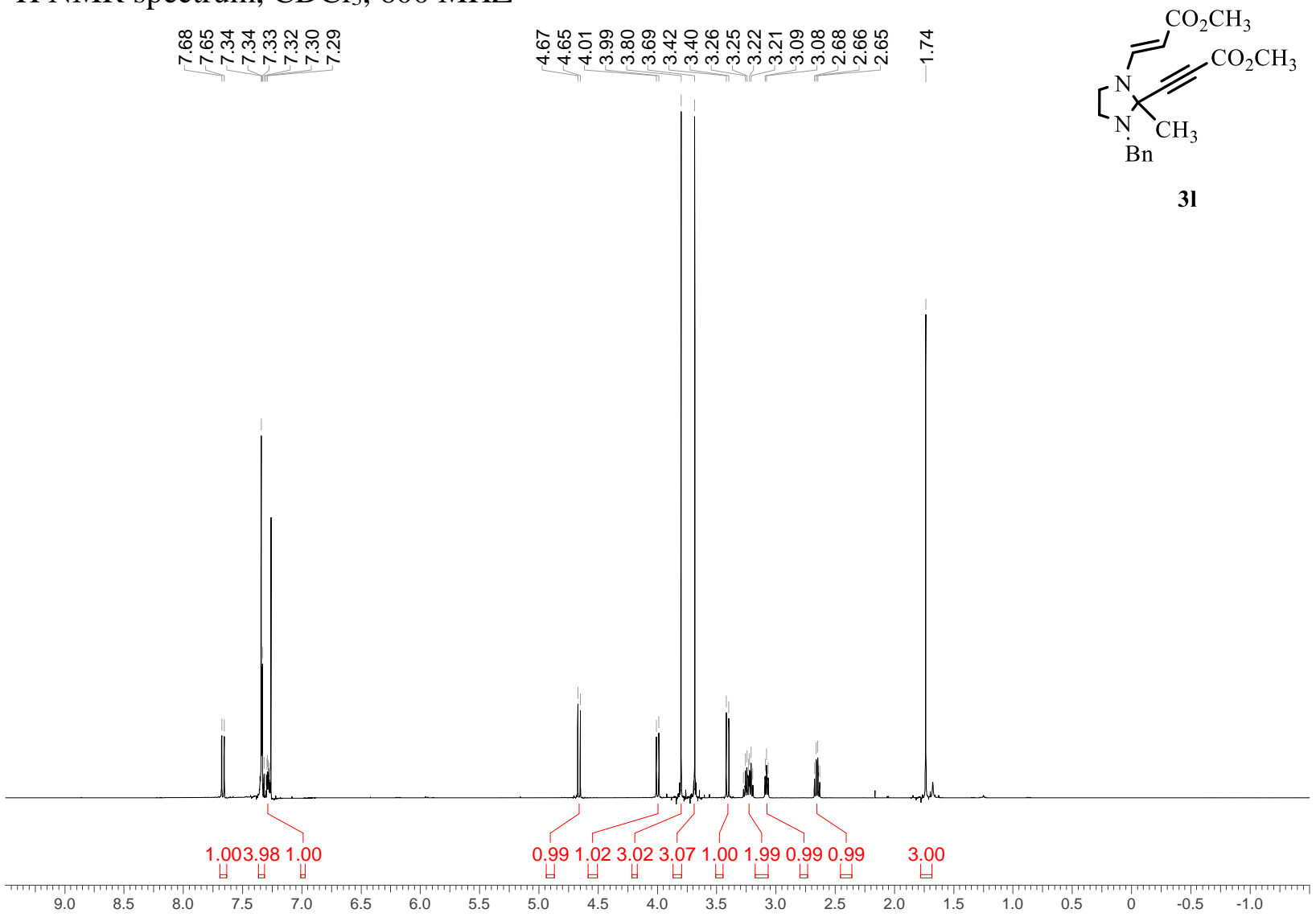

${ }^{13} \mathrm{C}$ NMR spectrum, $\mathrm{CDCl}_{3}, 150 \mathrm{MHz}$

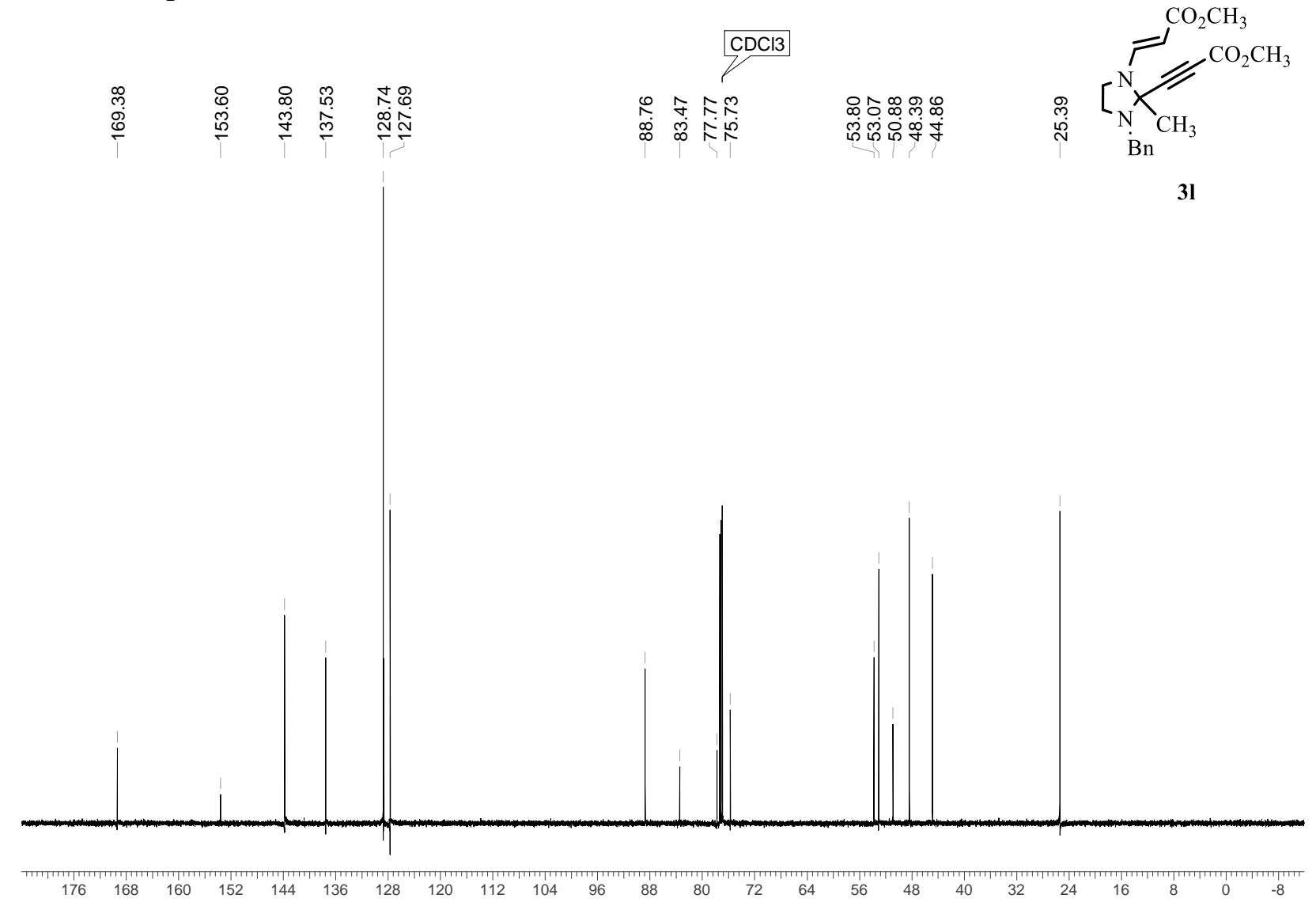


${ }^{1} \mathrm{H}$ NMR spectrum, $\mathrm{CDCl}_{3}, 600 \mathrm{MHZ}$

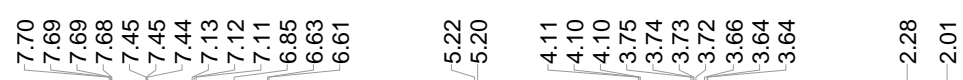

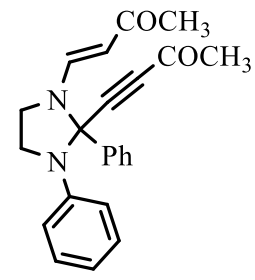

$3 \mathrm{~m}$

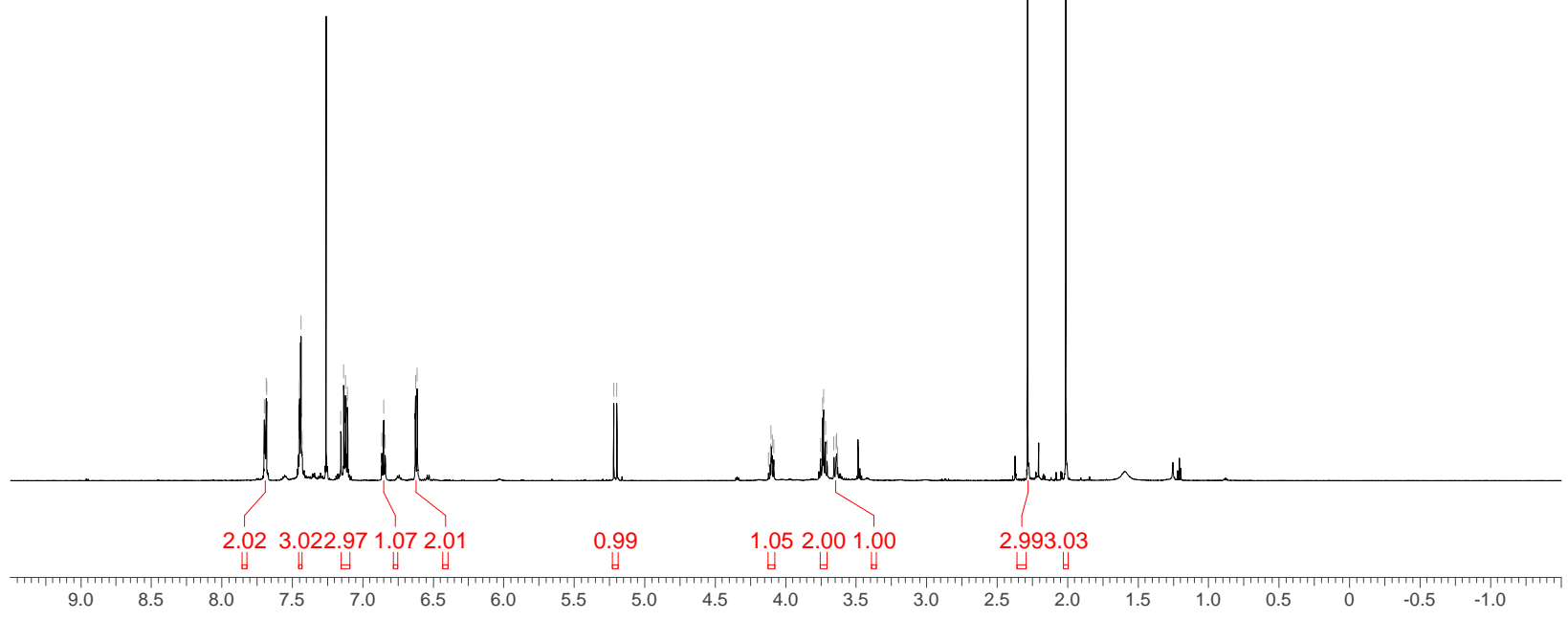

${ }^{13} \mathrm{C}$ NMR spectrum, $\mathrm{CDCl}_{3}, 150 \mathrm{MHz}$

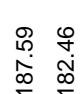

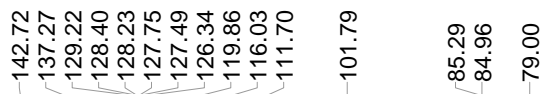

-

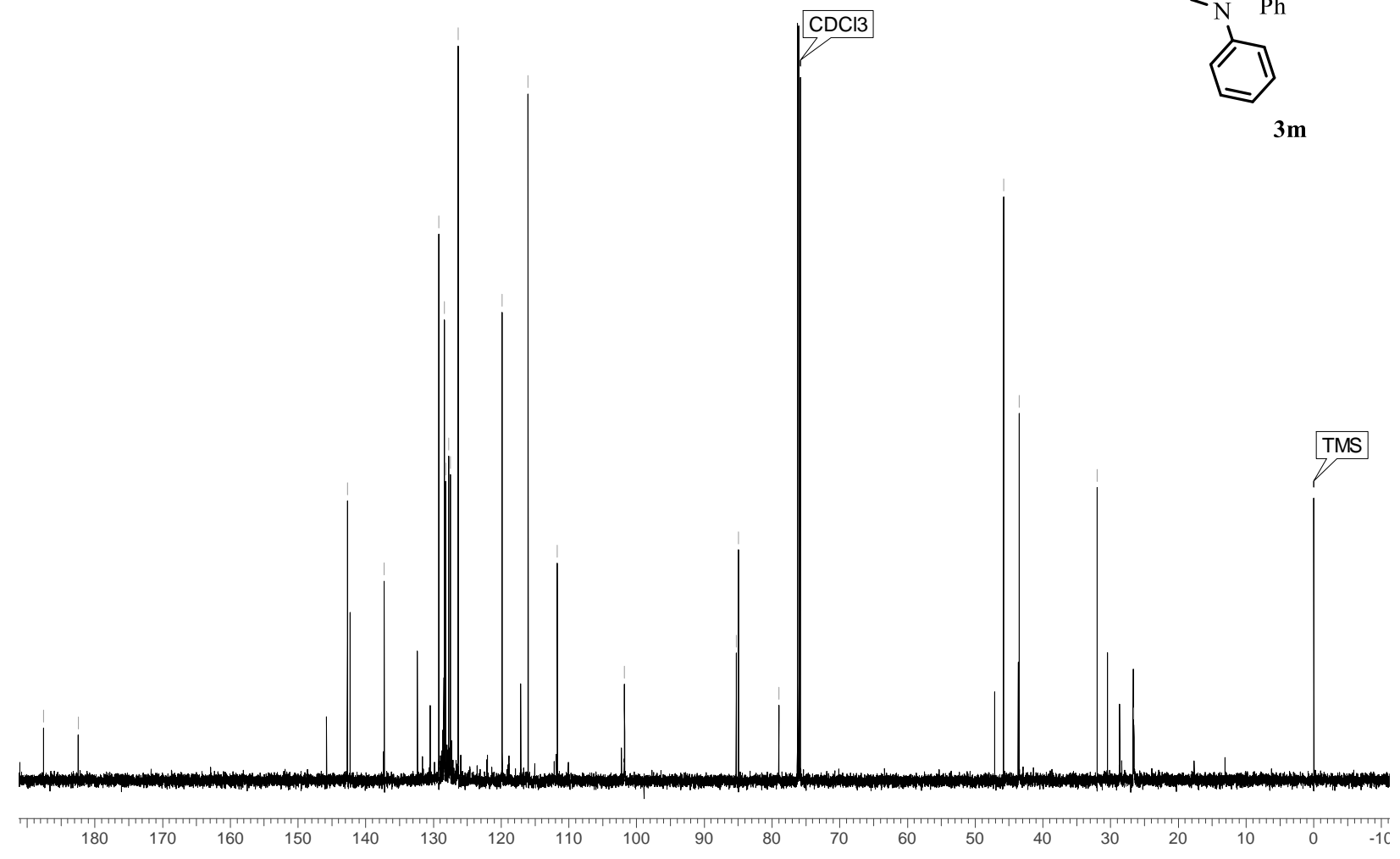


${ }^{1} \mathrm{H}$ NMR spectrum, $\mathrm{CDCl}_{3}, 600 \mathrm{MHZ}$

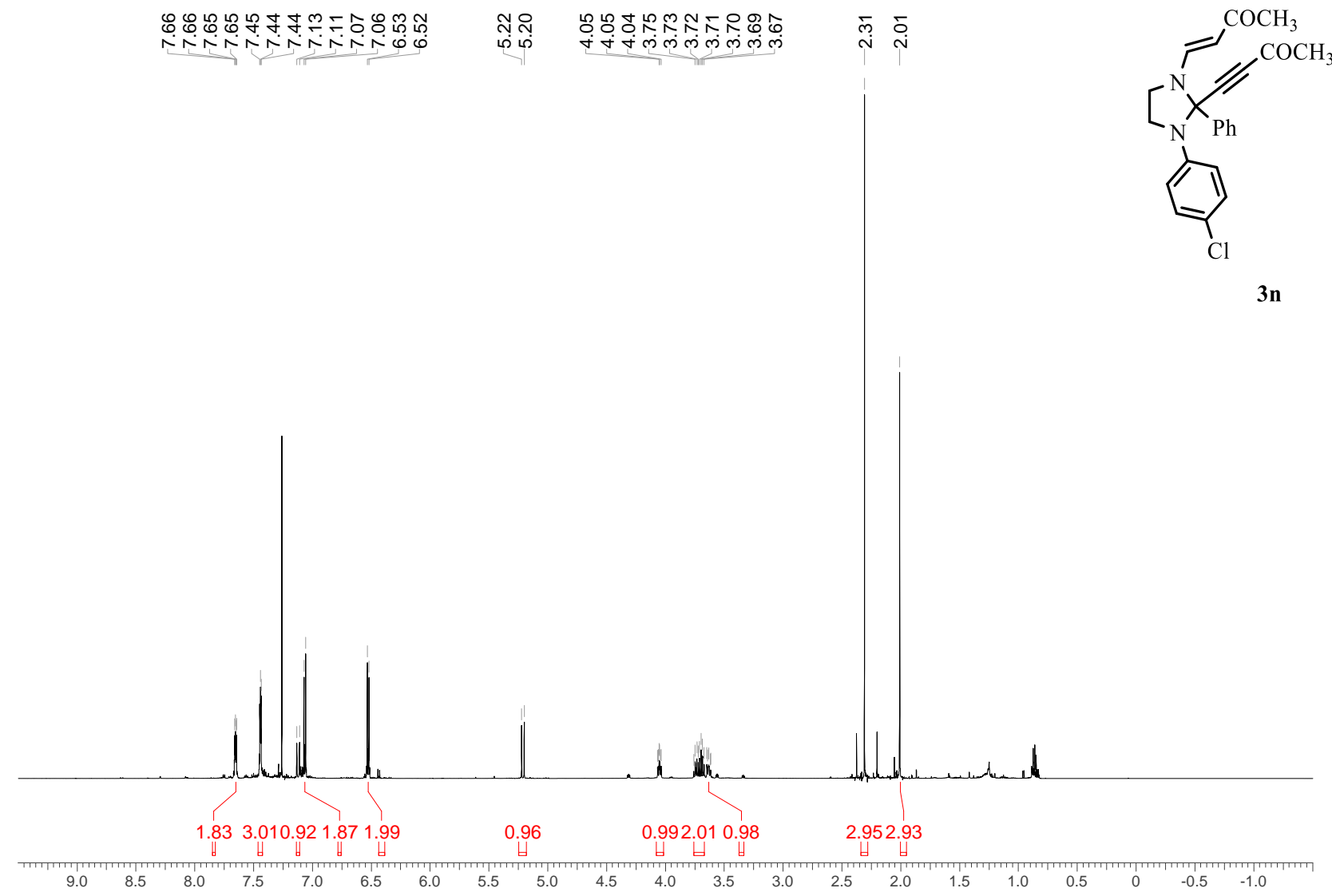

${ }^{13} \mathrm{C}$ NMR spectrum, $\mathrm{CDCl}_{3}, 150 \mathrm{MHz}$

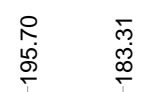

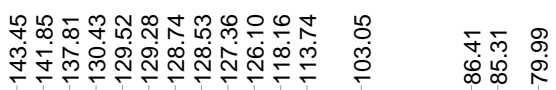

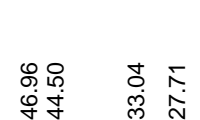
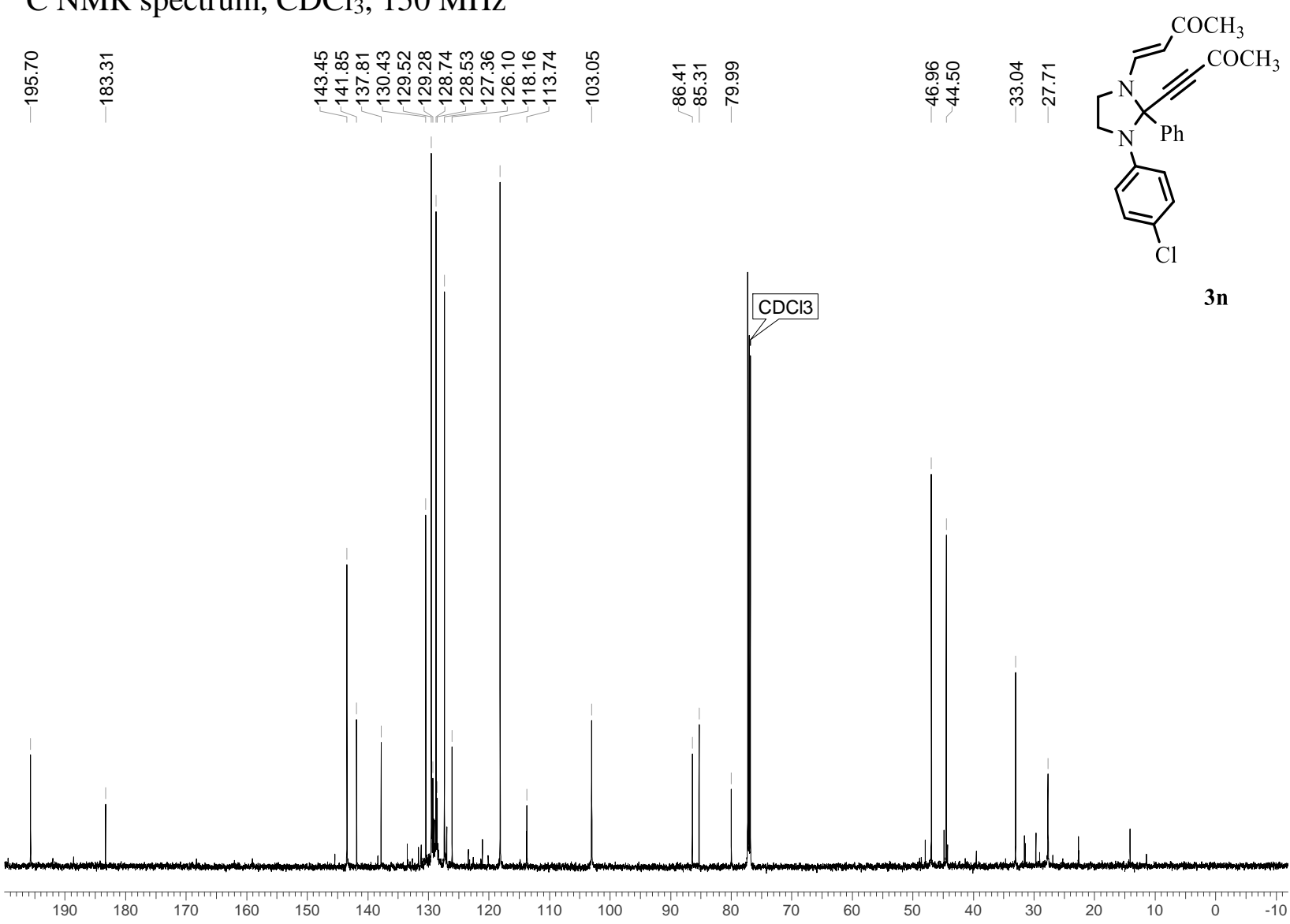
${ }^{1} \mathrm{H}$ NMR spectrum, $\mathrm{CDCl}_{3}, 600 \mathrm{MHZ}$

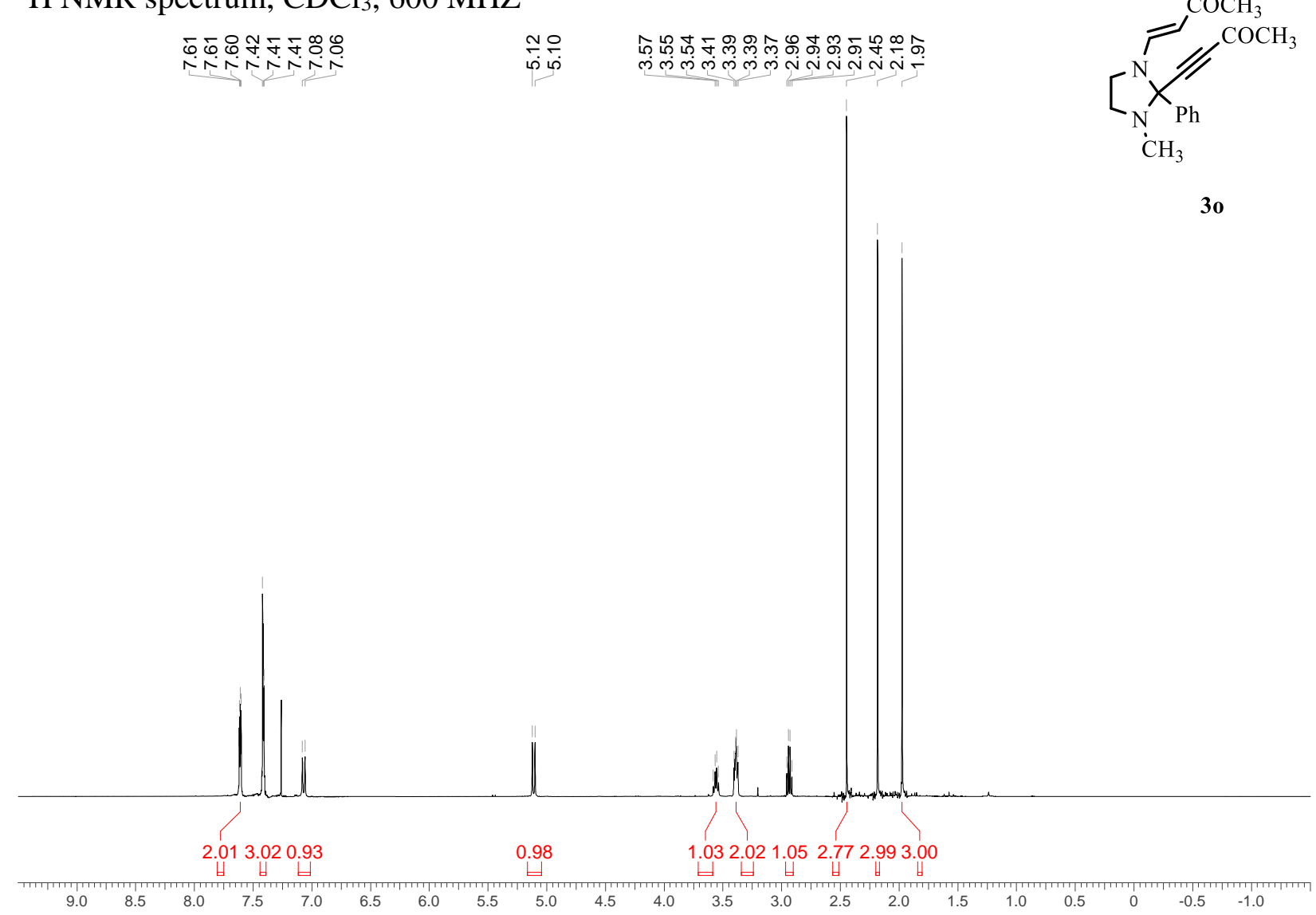

${ }^{13} \mathrm{C}$ NMR spectrum, $\mathrm{CDCl}_{3}, 150 \mathrm{MHz}$

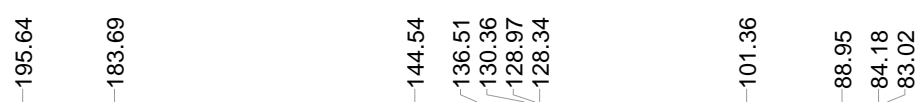

\&

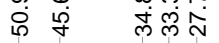

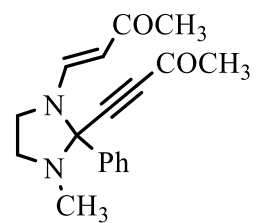

30

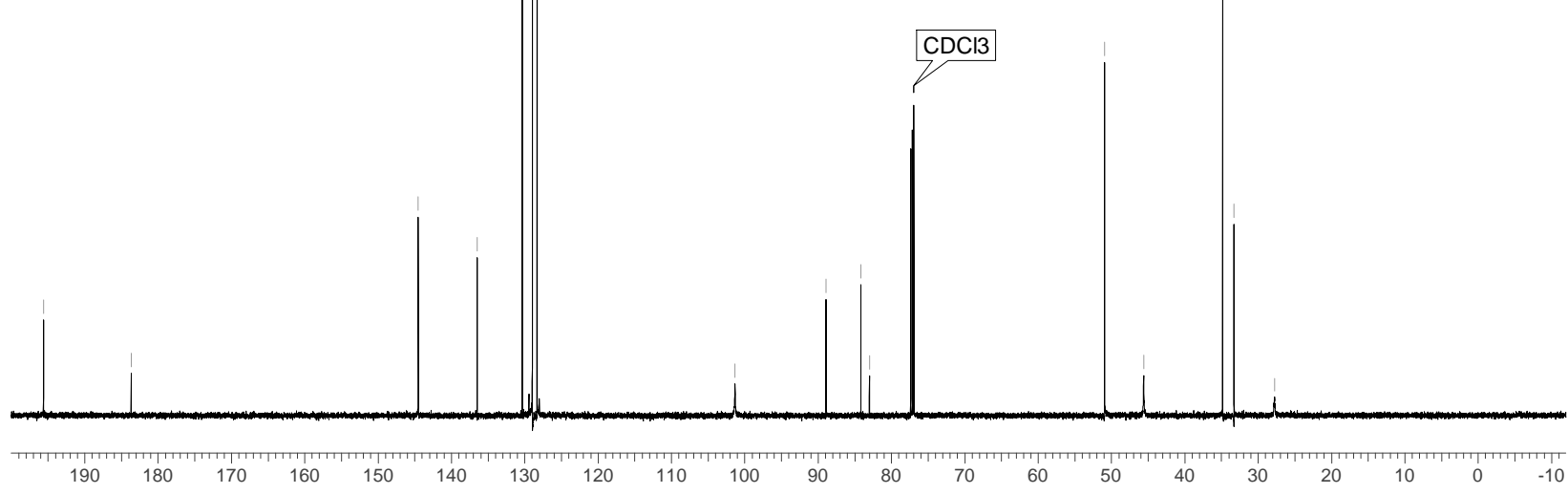


${ }^{1} \mathrm{H}$ NMR spectrum, $\mathrm{CDCl}_{3}, 600 \mathrm{MHZ}$

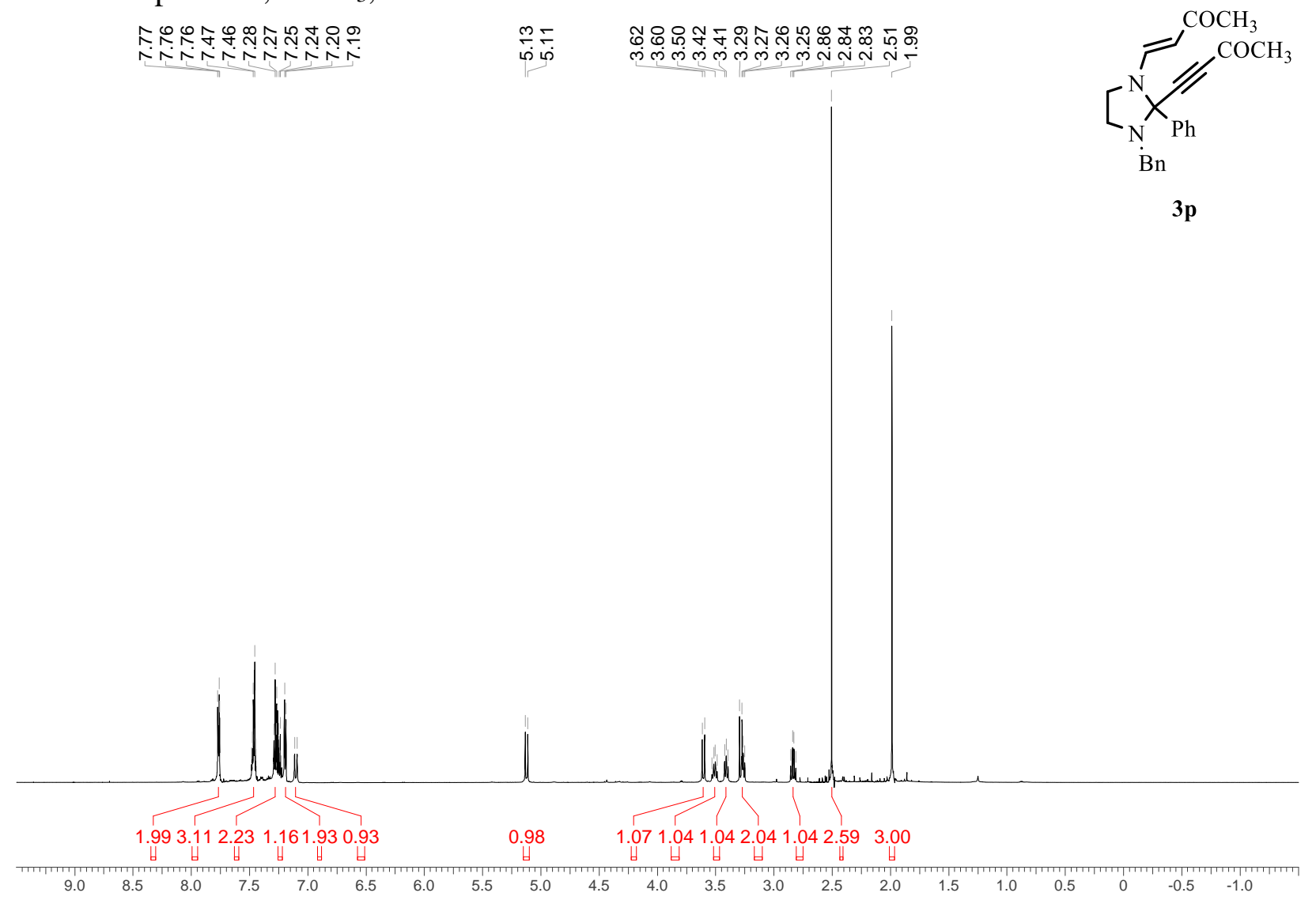

${ }^{13} \mathrm{C}$ NMR spectrum, $\mathrm{CDCl}_{3}, 150 \mathrm{MHz}$

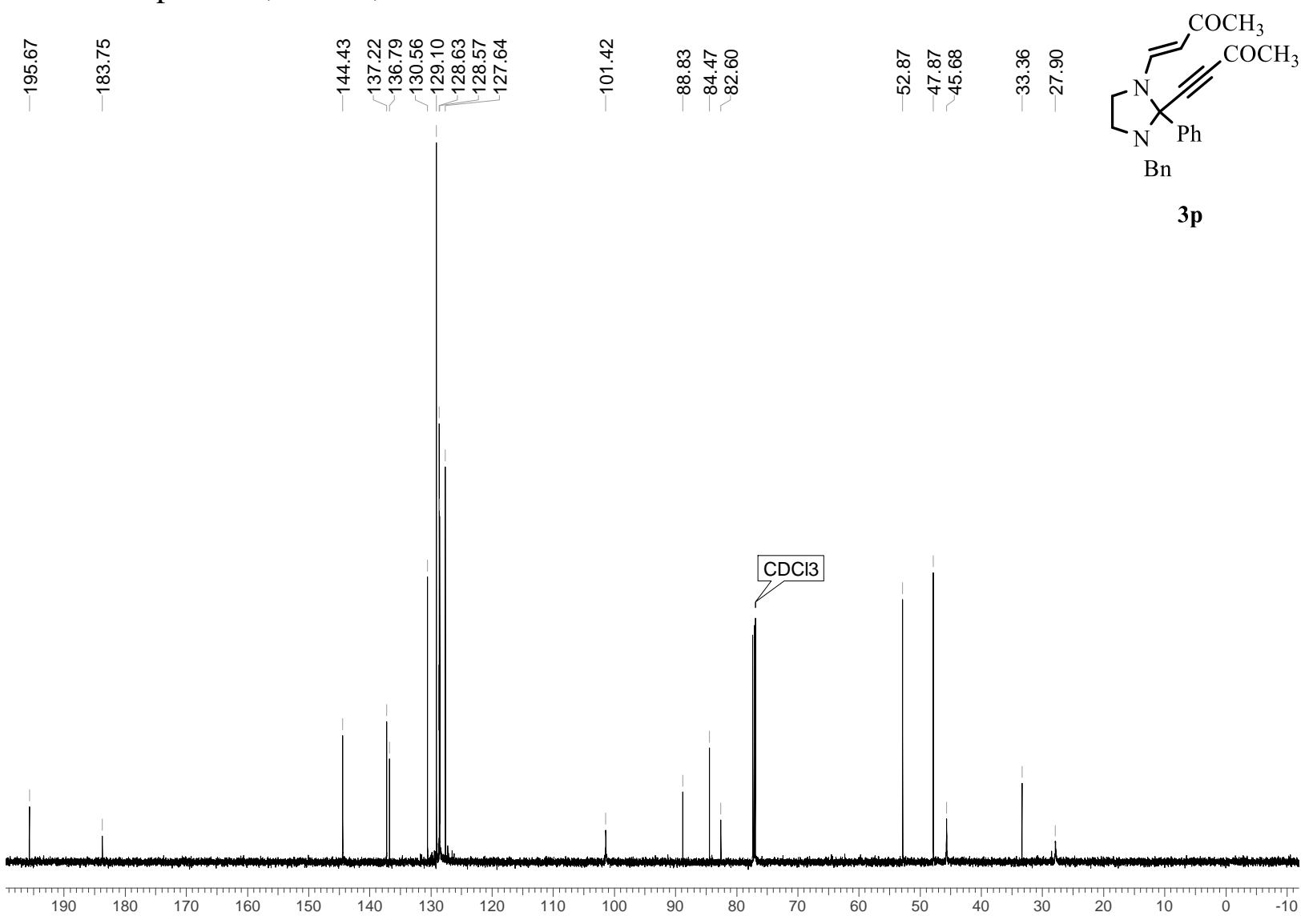


${ }^{1} \mathrm{H}$ NMR spectrum, $\mathrm{CDCl}_{3}, 600 \mathrm{MHZ}$

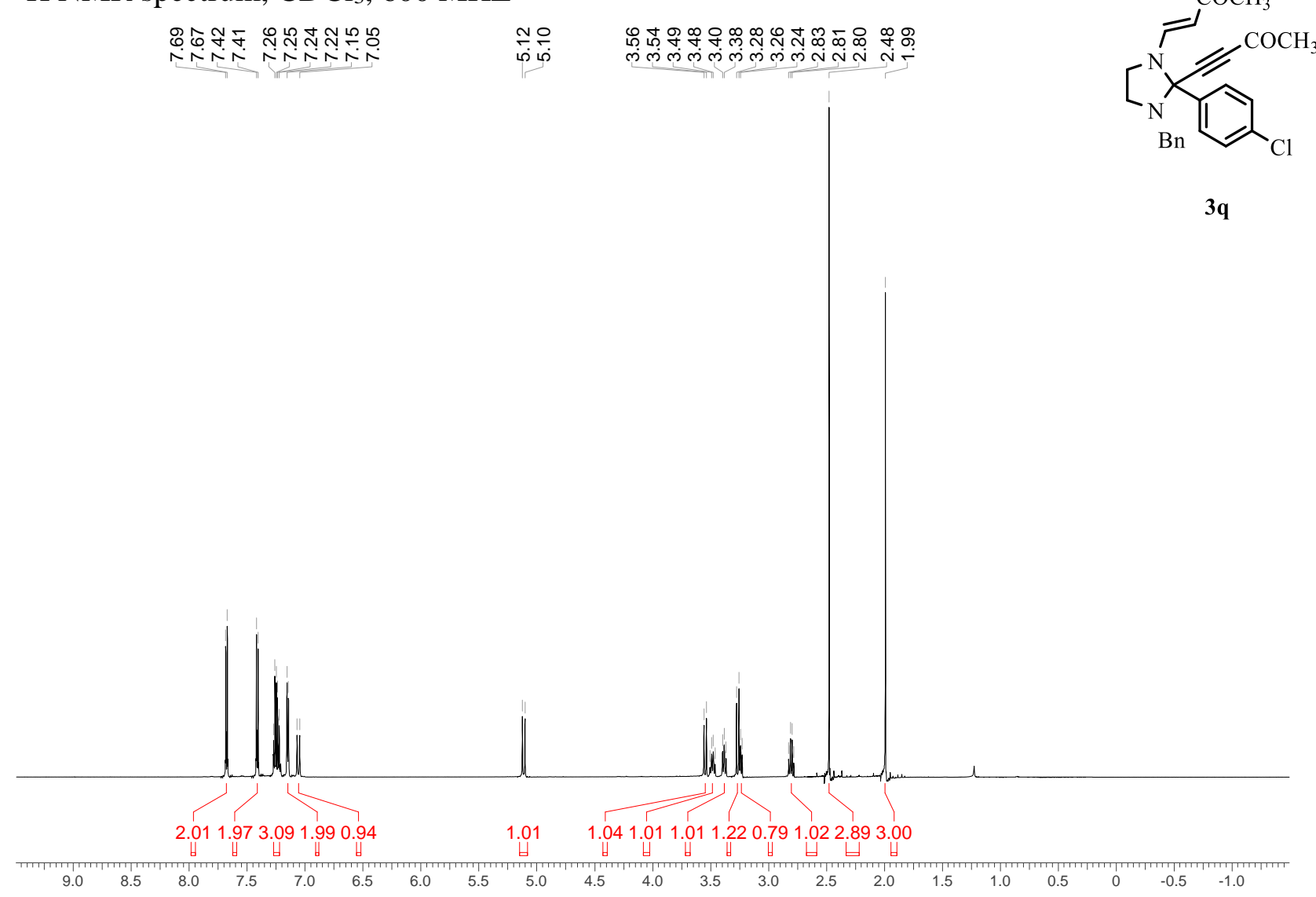

${ }^{13} \mathrm{C}$ NMR spectrum, $\mathrm{CDCl}_{3}, 150 \mathrm{MHz}$

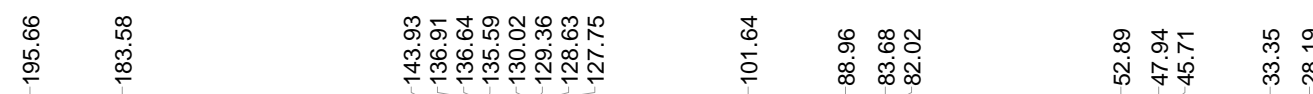<smiles>CC(=O)C=CN(C=CC(C)=O)C1(c2ccc(Cl)cc2)CCNC1</smiles>

$3 q$

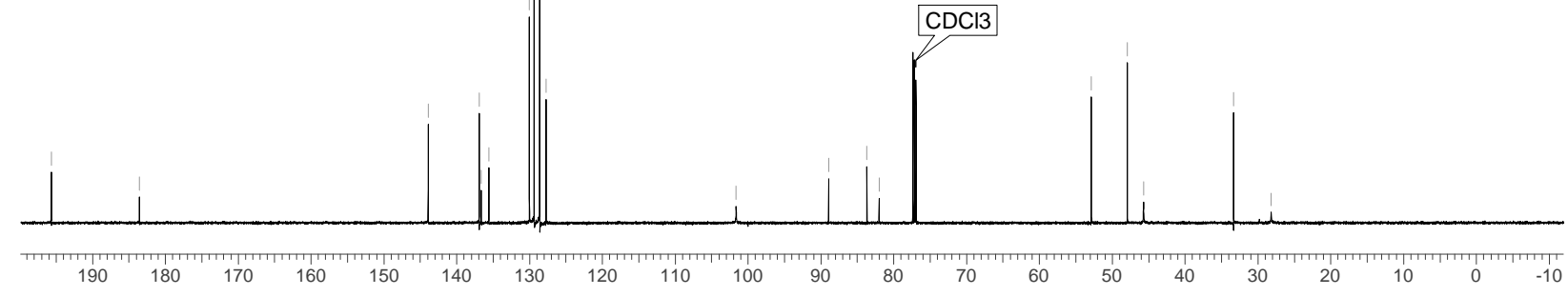


${ }^{1} \mathrm{H}$ NMR spectrum, $\mathrm{CDCl}_{3}, 600 \mathrm{MHZ}$

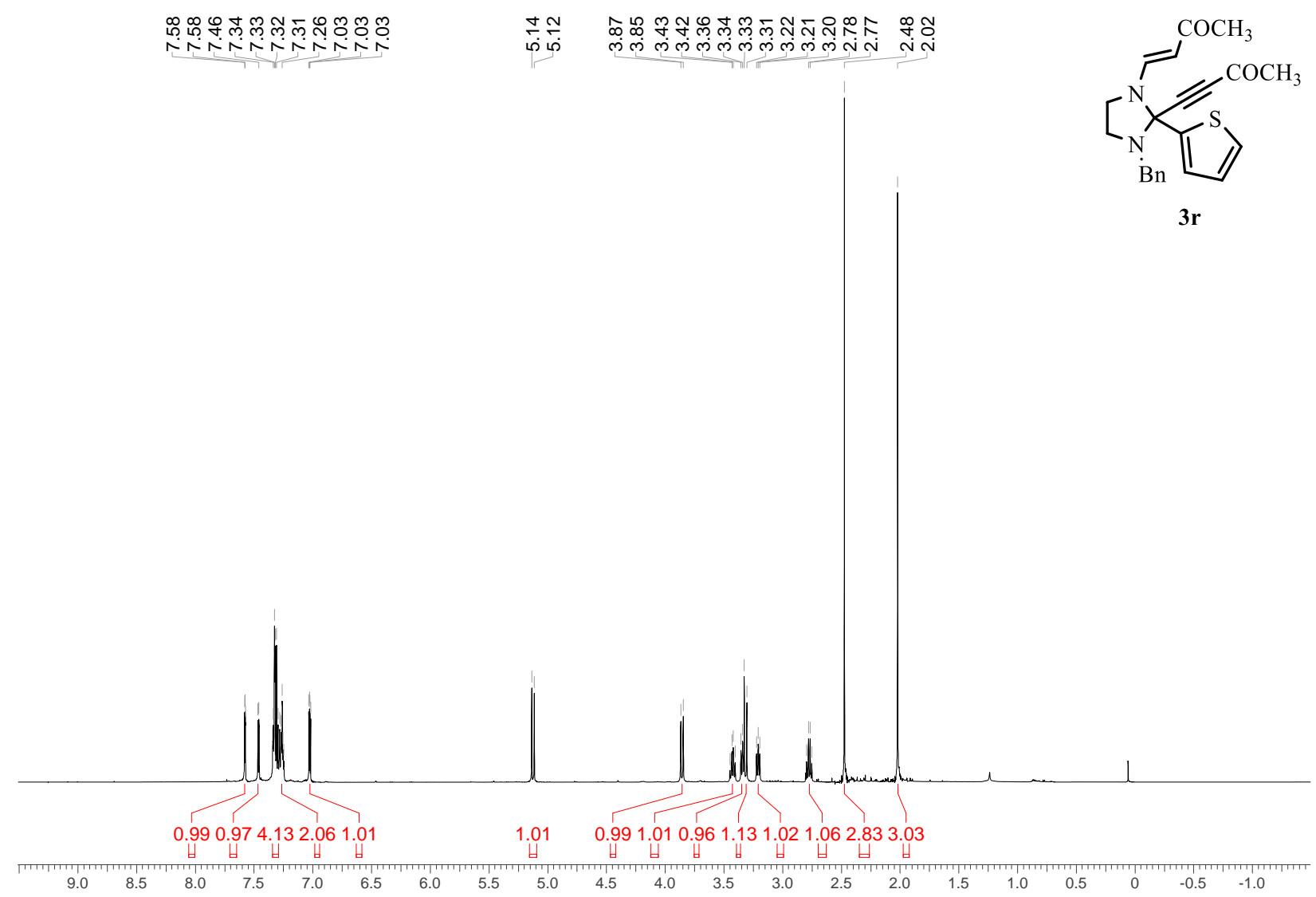

${ }^{13} \mathrm{C}$ NMR spectrum, $\mathrm{CDCl}_{3}, 150 \mathrm{MHz}$

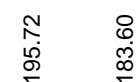

추ㅇㅠㅠㅇㅠ

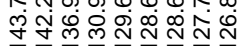

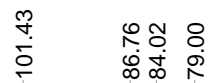

啇

ำ

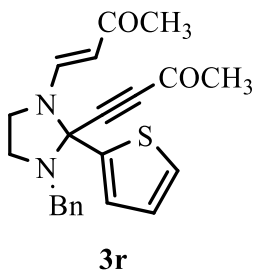

$3 \mathbf{r}$

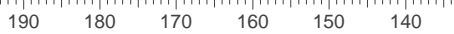


${ }^{1} \mathrm{H}$ NMR spectrum, $\mathrm{CDCl}_{3}, 600 \mathrm{MHZ}$

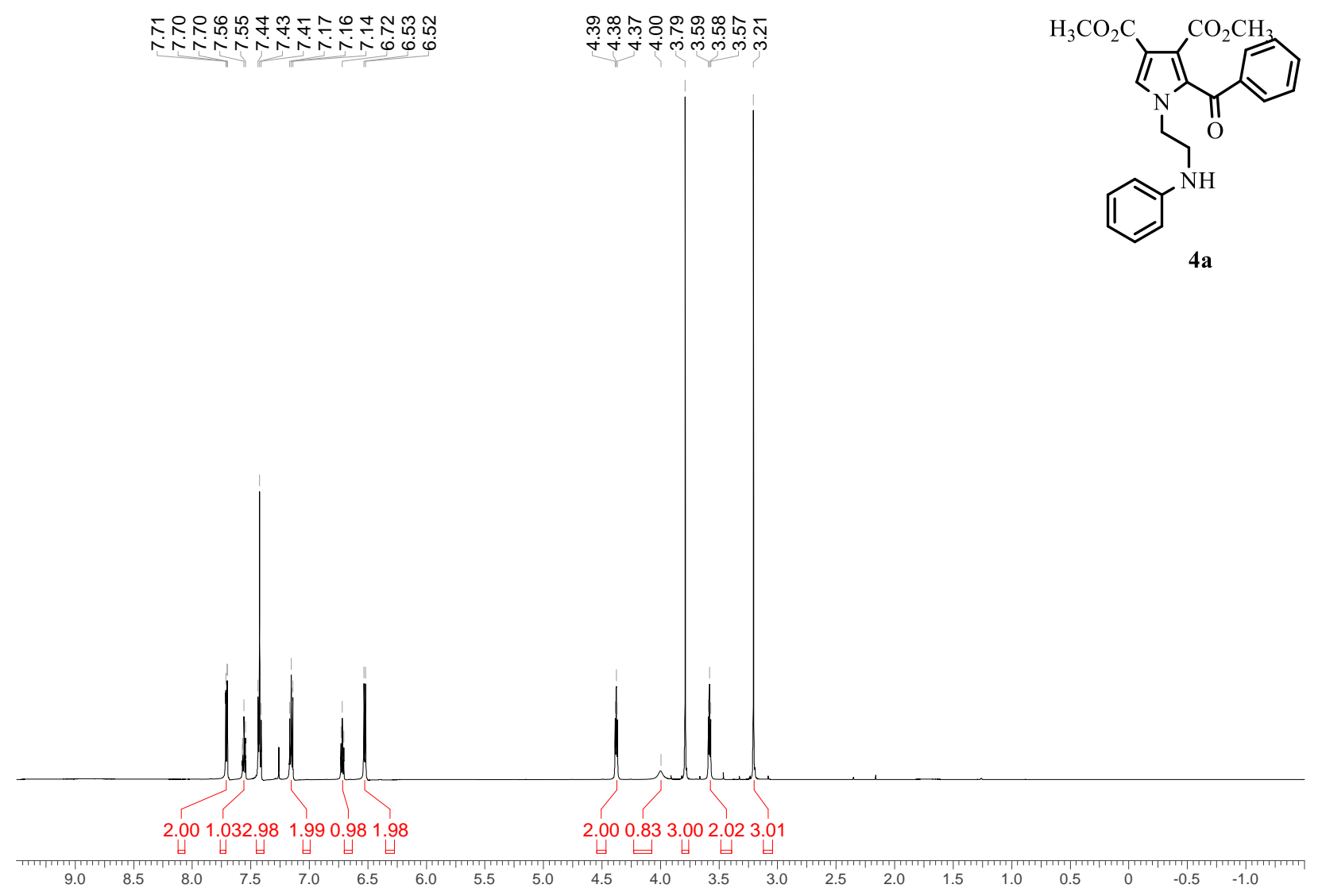

${ }^{13} \mathrm{C}$ NMR spectrum, $\mathrm{CDCl}_{3}, 150 \mathrm{MHz}$

万.

每
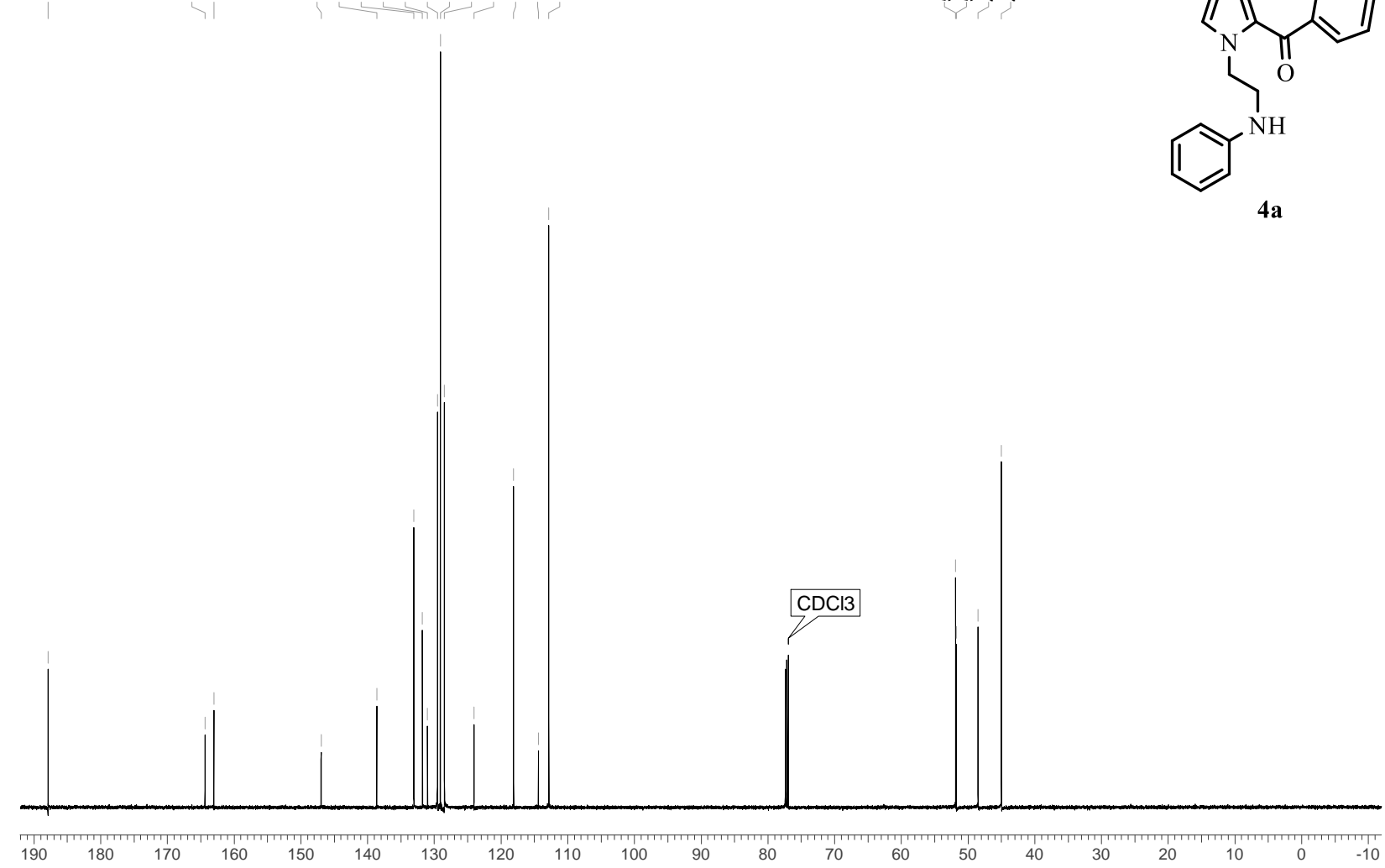
${ }^{1} \mathrm{H}$ NMR spectrum, $\mathrm{CDCl}_{3}, 600 \mathrm{MHZ}$

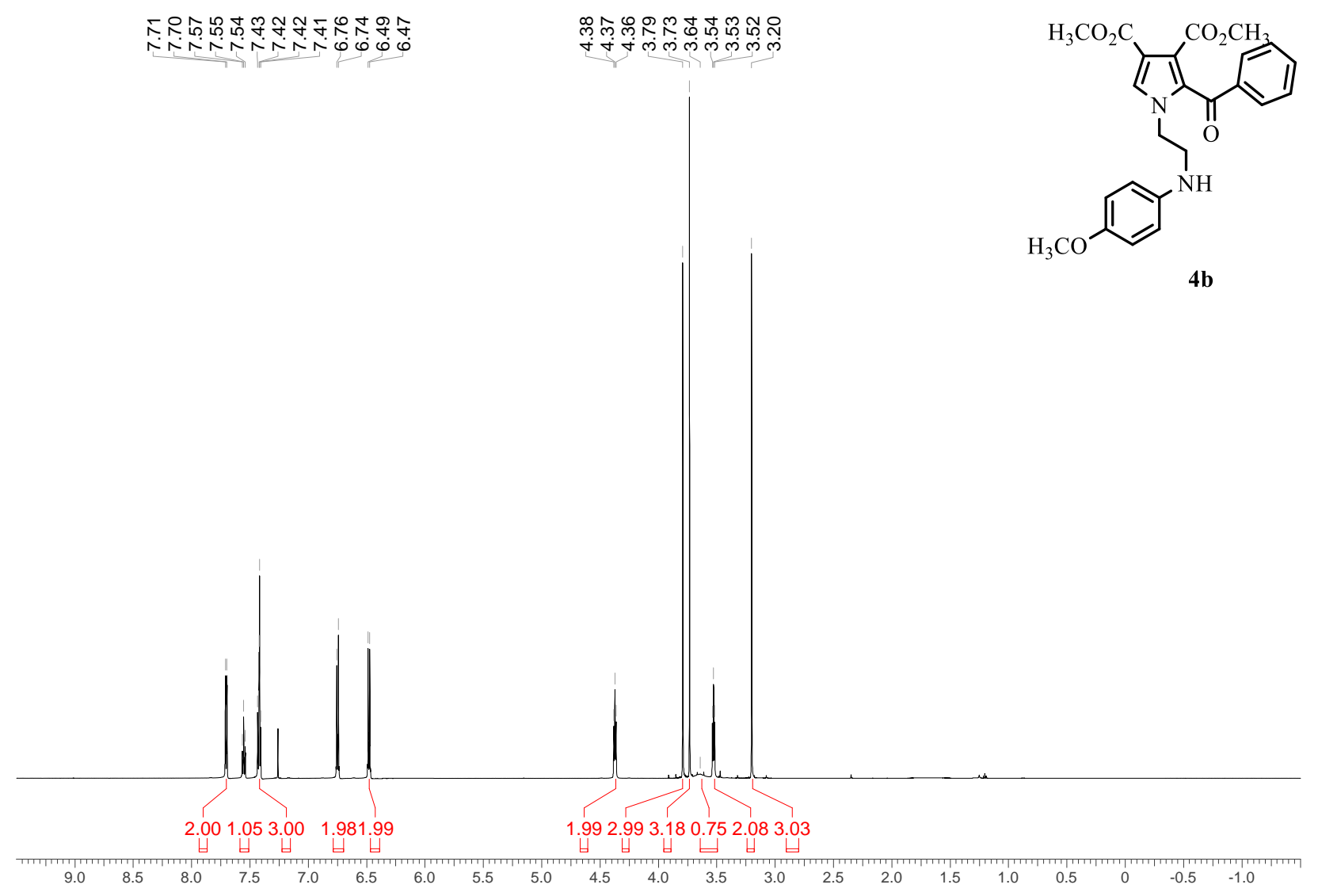

${ }^{13} \mathrm{C}$ NMR spectrum, $\mathrm{CDCl}_{3}, 150 \mathrm{MHz}$

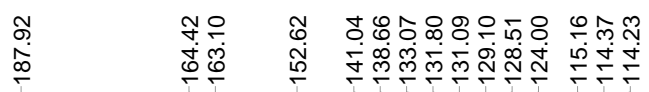

\begin{tabular}{|l|l|}
$\mathrm{CDCl}$ \\
\hline
\end{tabular}

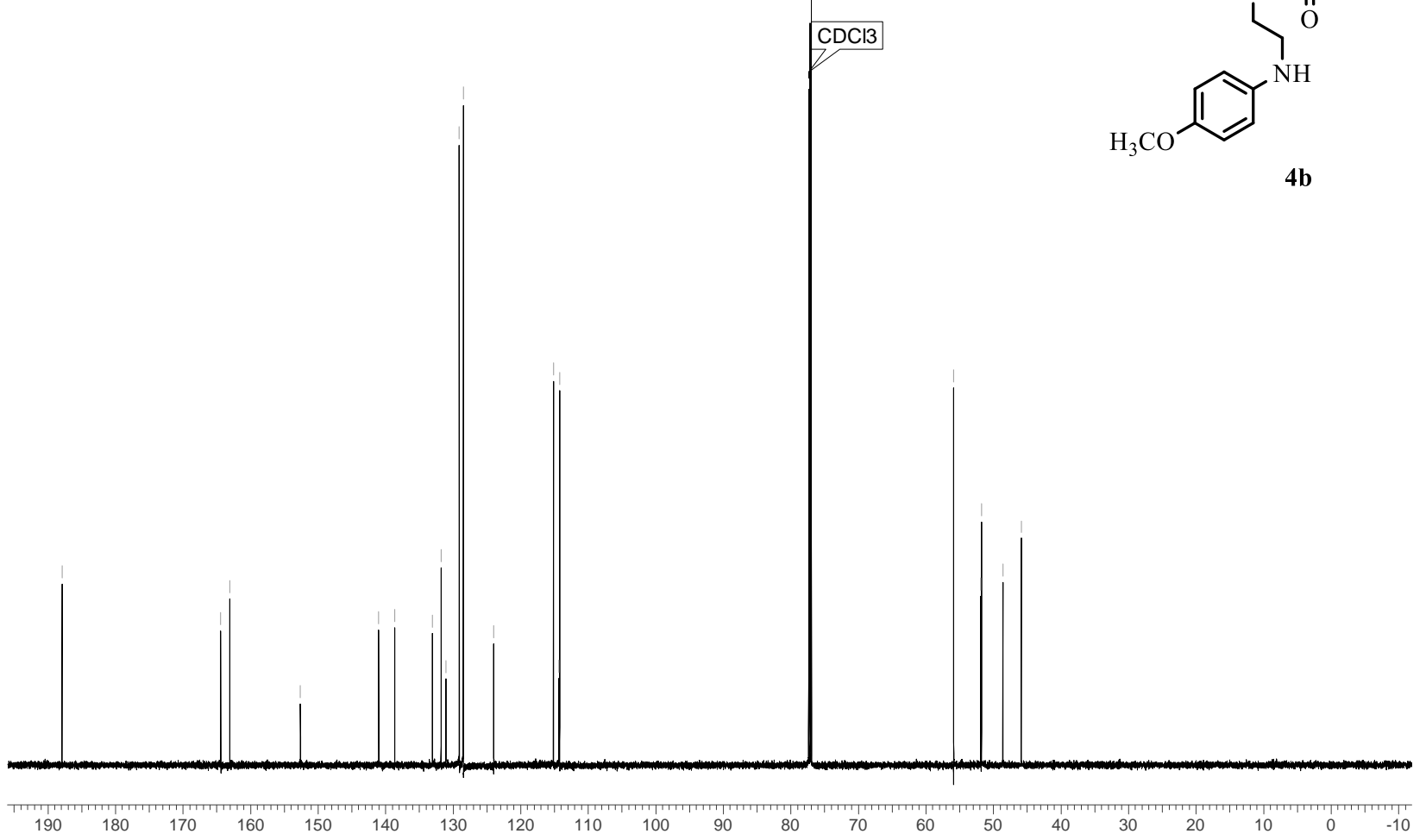


${ }^{1} \mathrm{H}$ NMR spectrum, $\mathrm{CDCl}_{3}, 600 \mathrm{MHZ}$

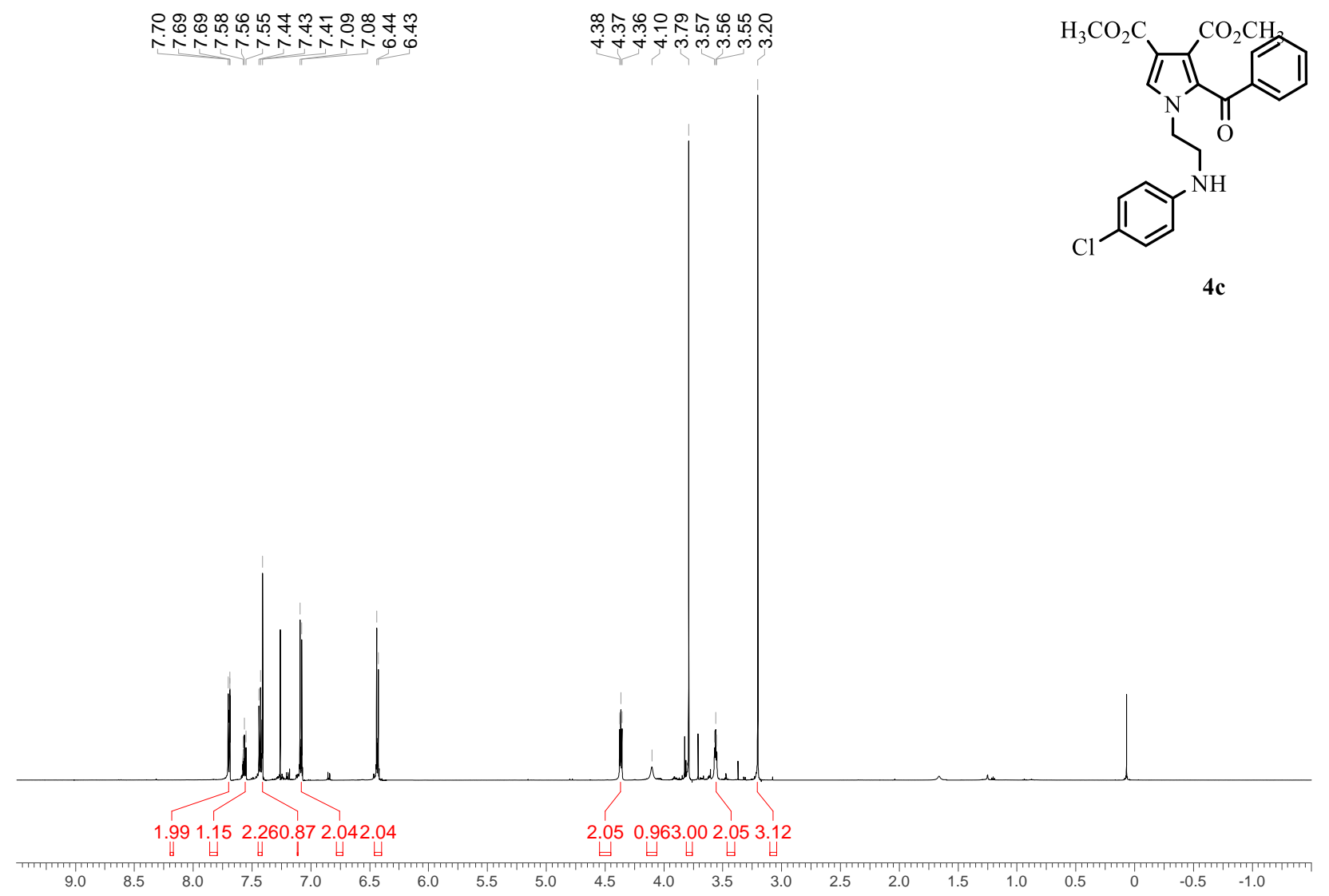

${ }^{13} \mathrm{C}$ NMR spectrum, $\mathrm{CDCl}_{3}, 150 \mathrm{MHz}$

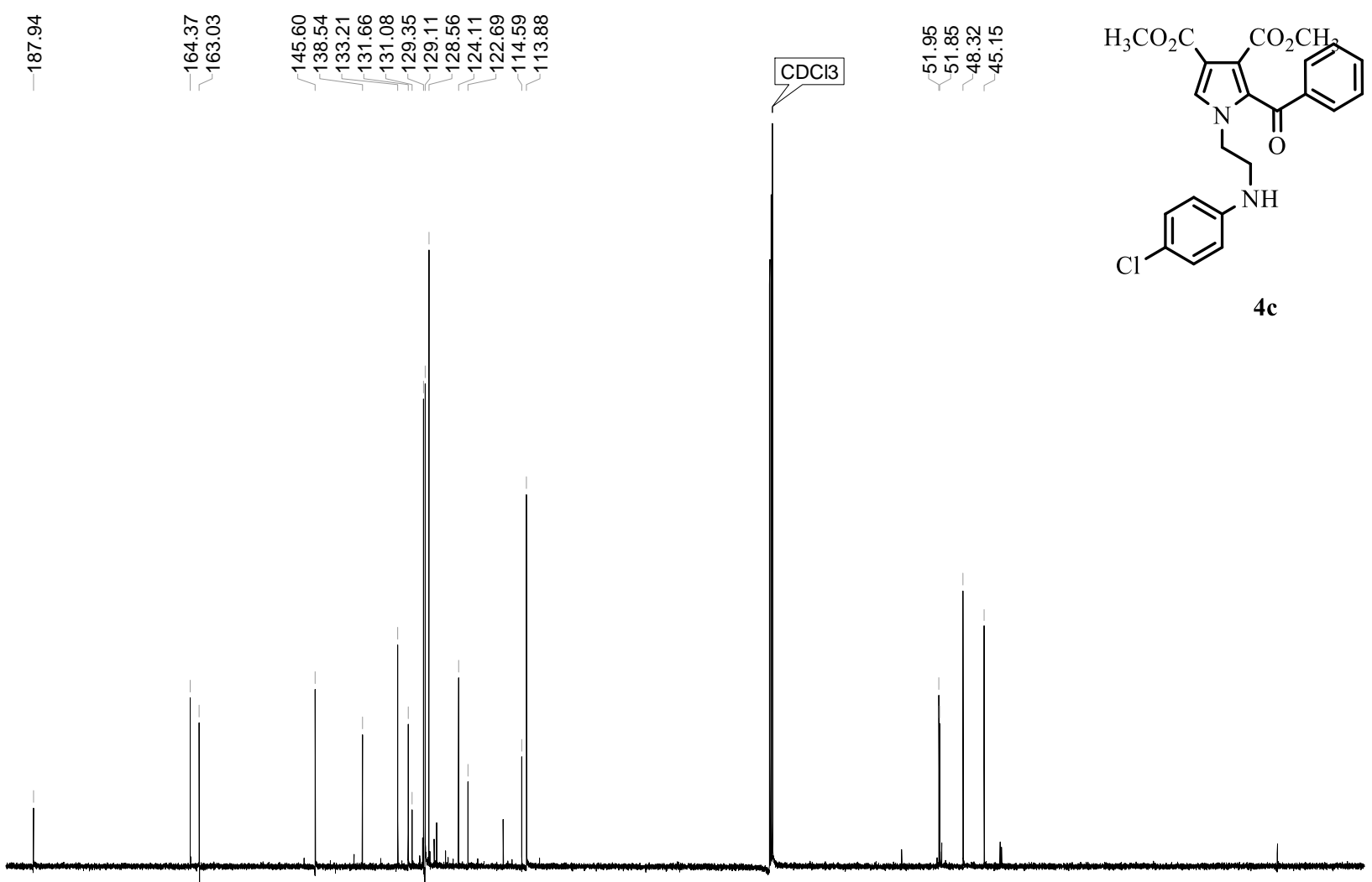

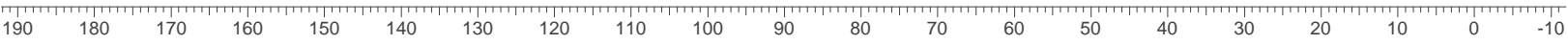


${ }^{1} \mathrm{H}$ NMR spectrum, $\mathrm{CDCl}_{3}, 600 \mathrm{MHZ}$

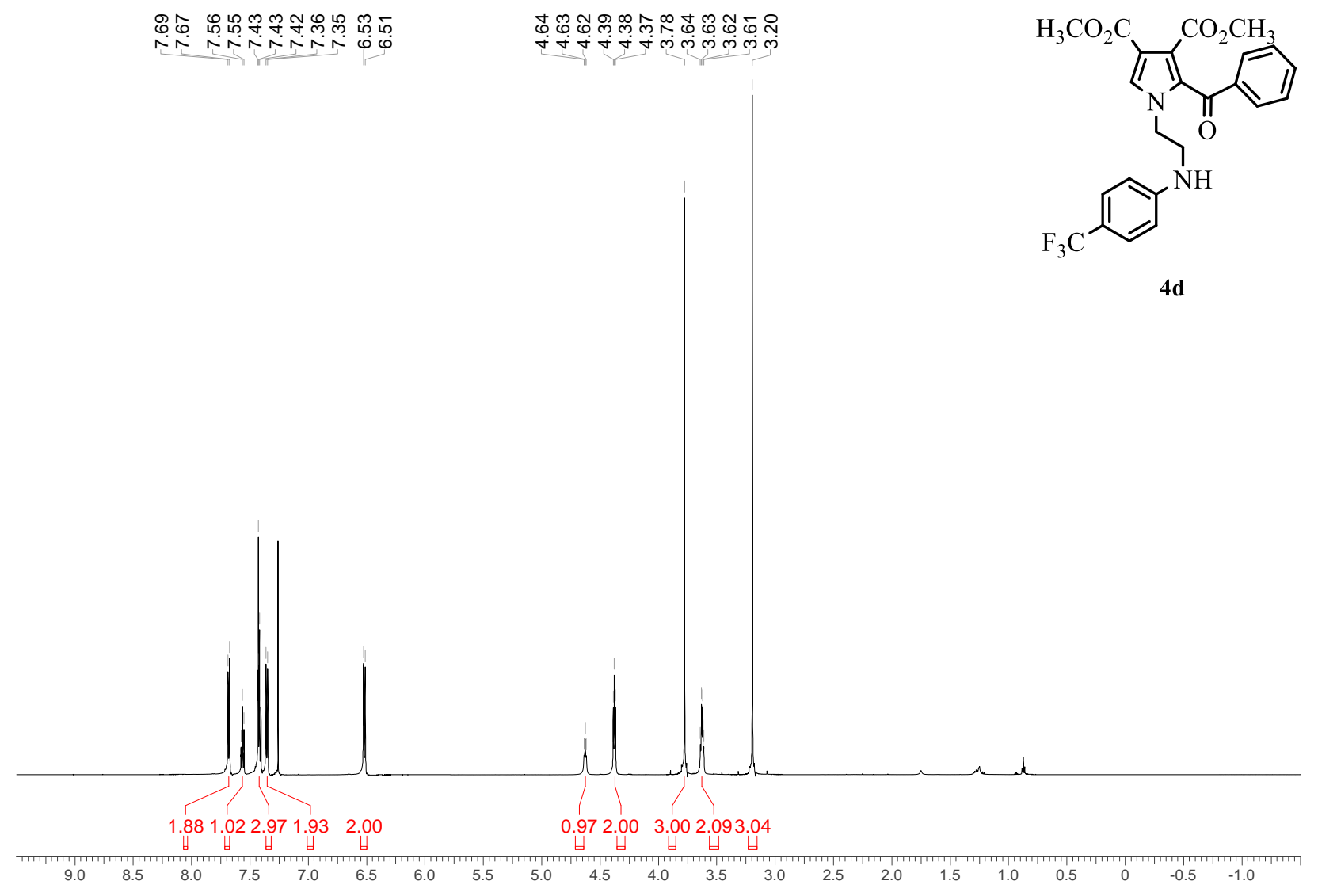

${ }^{13} \mathrm{C}$ NMR spectrum, $\mathrm{CDCl}_{3}, 150 \mathrm{MHz}$
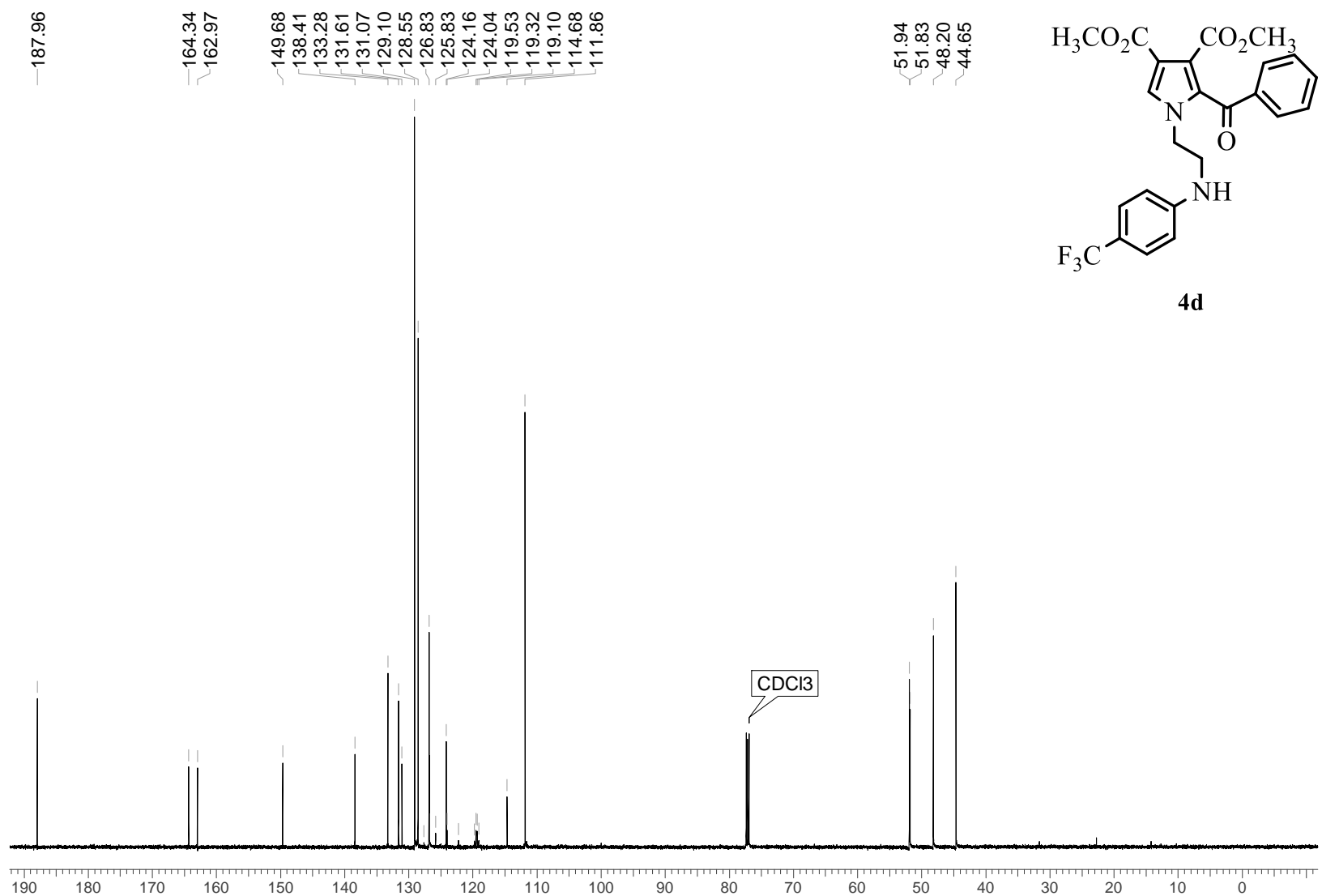
${ }^{1} \mathrm{H}$ NMR spectrum, $\mathrm{CDCl}_{3}, 600 \mathrm{MHZ}$

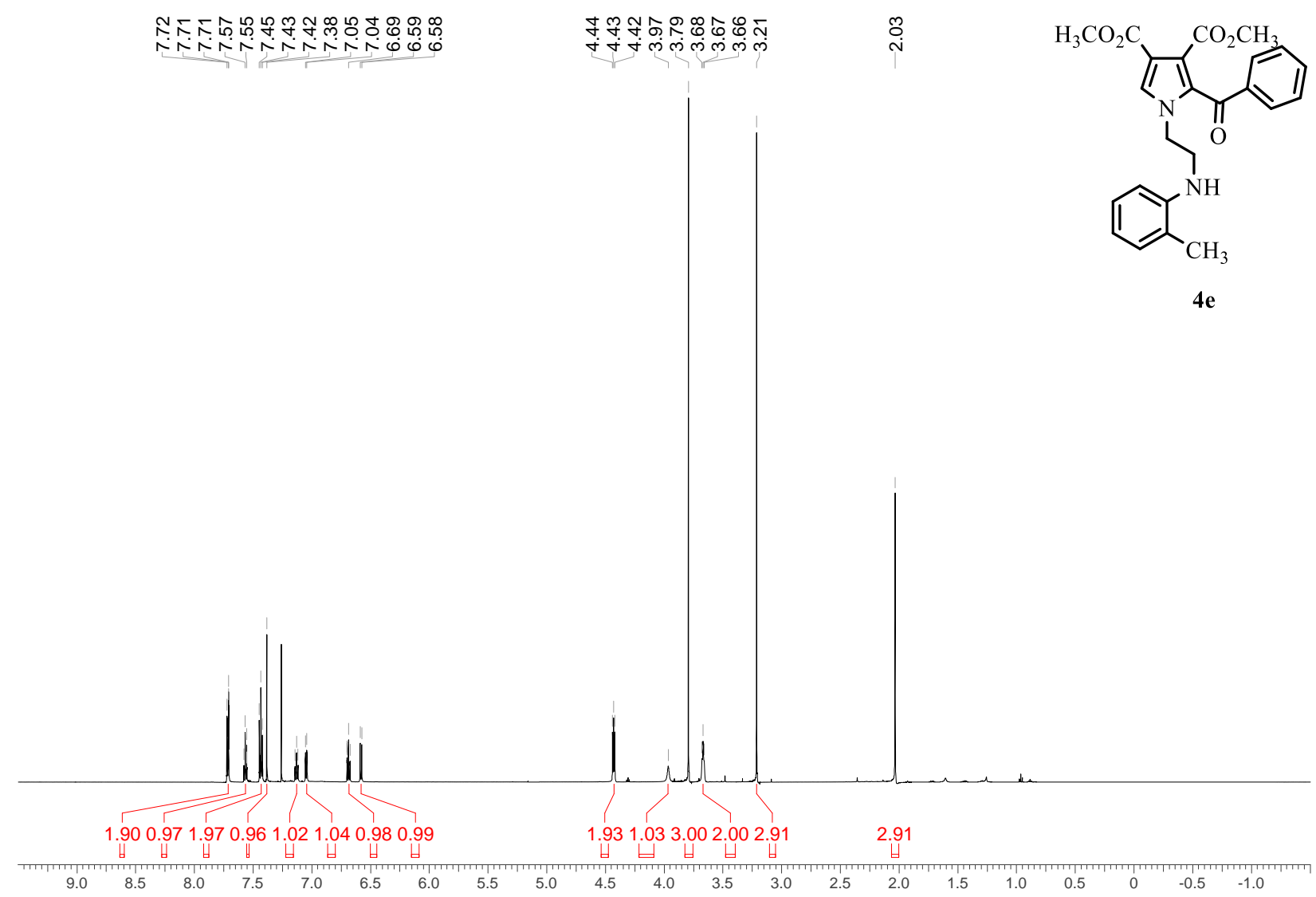

${ }^{13} \mathrm{C}$ NMR spectrum, $\mathrm{CDCl}_{3}, 150 \mathrm{MHz}$
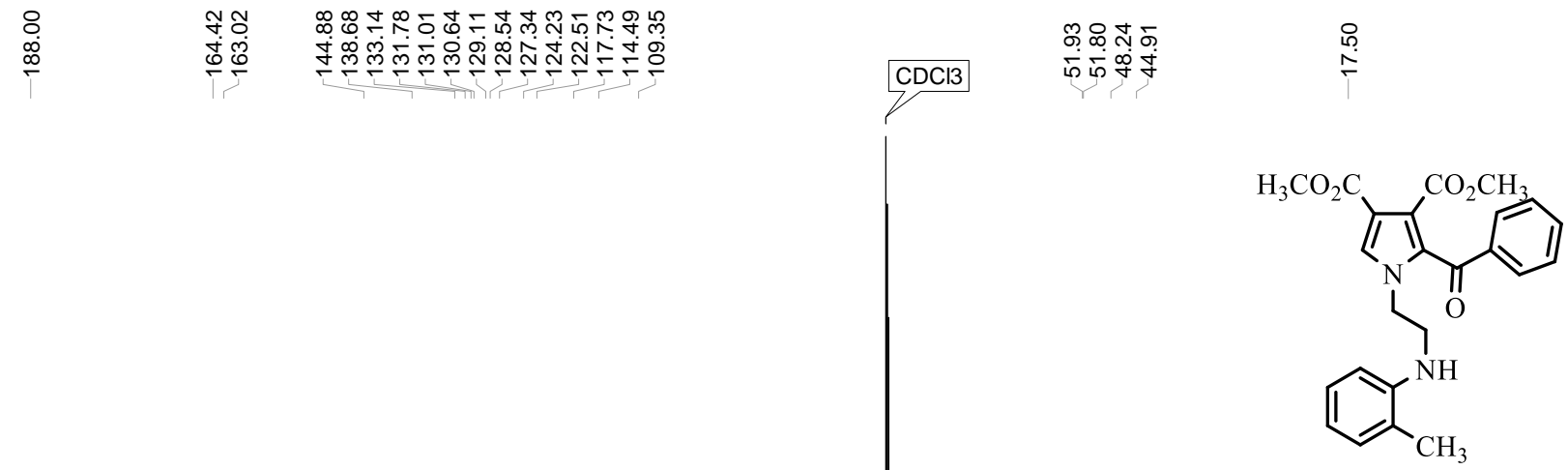

$4 e$

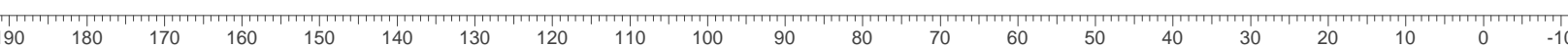


${ }^{1} \mathrm{H}$ NMR spectrum, $\mathrm{CDCl}_{3}, 600 \mathrm{MHZ}$

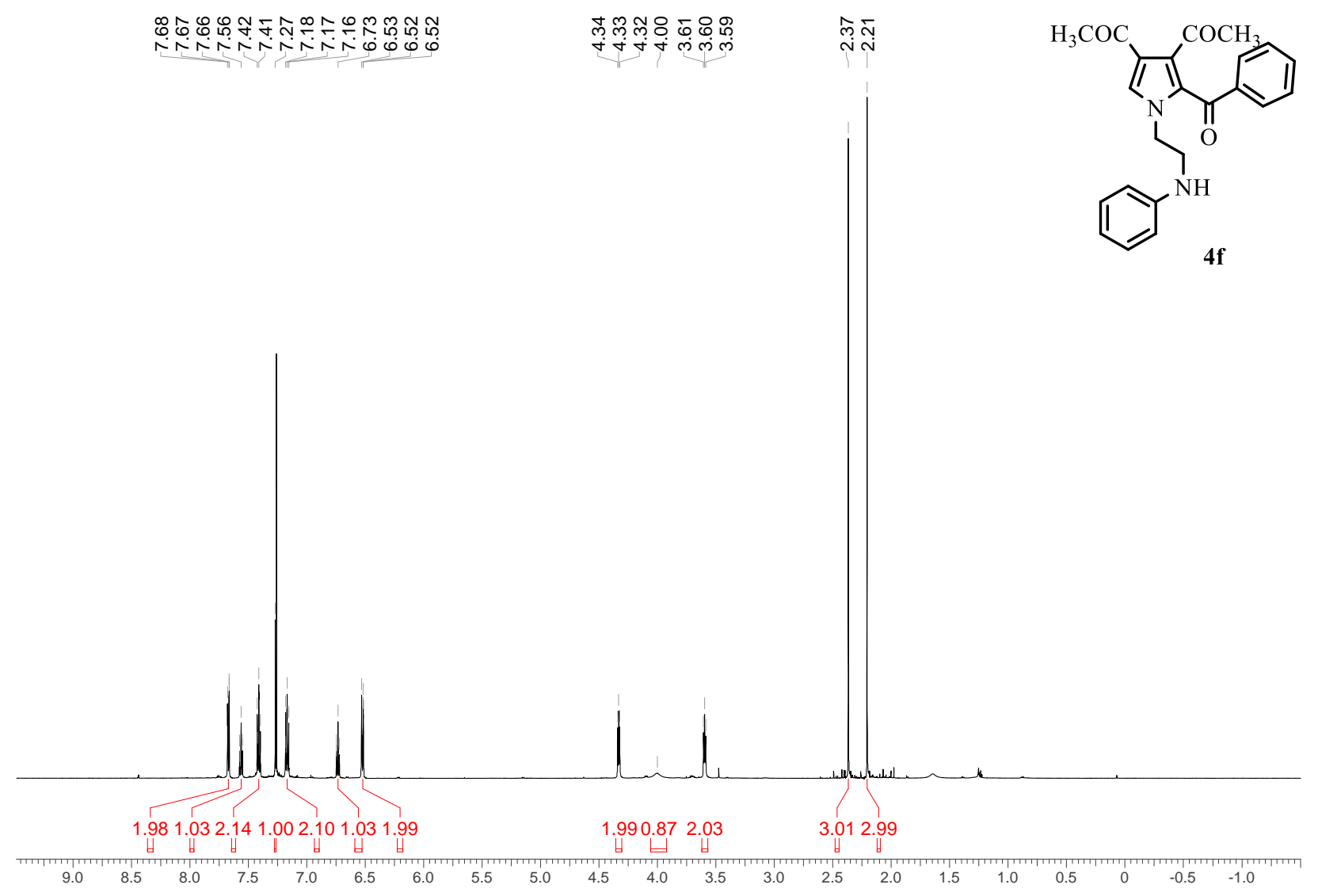

${ }^{13} \mathrm{C}$ NMR spectrum, $\mathrm{CDCl}_{3}, 150 \mathrm{MHz}$

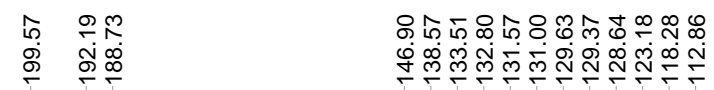

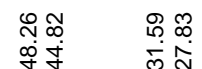

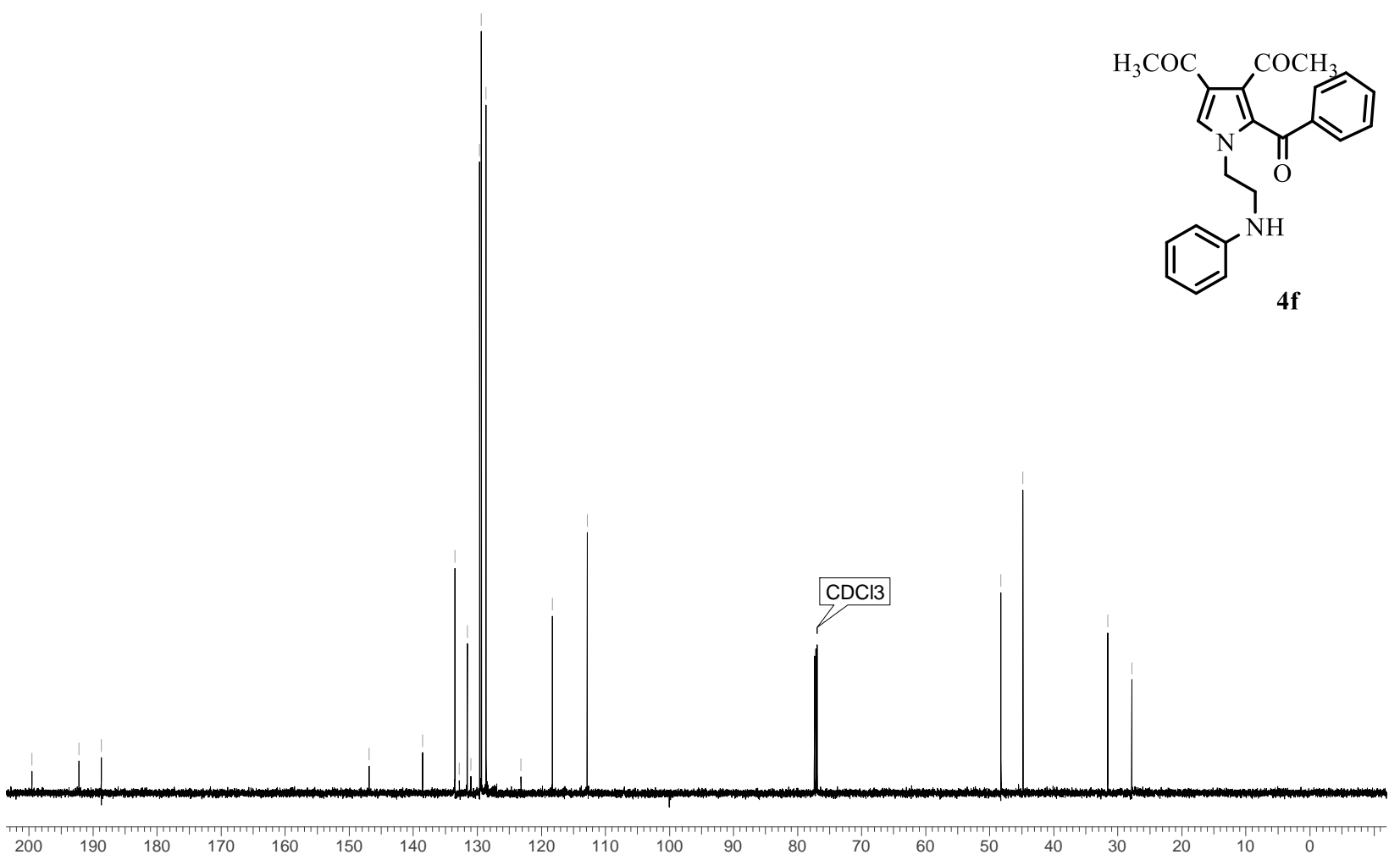


${ }^{1} \mathrm{H}$ NMR spectrum, $\mathrm{CDCl}_{3}, 600 \mathrm{MHZ}$

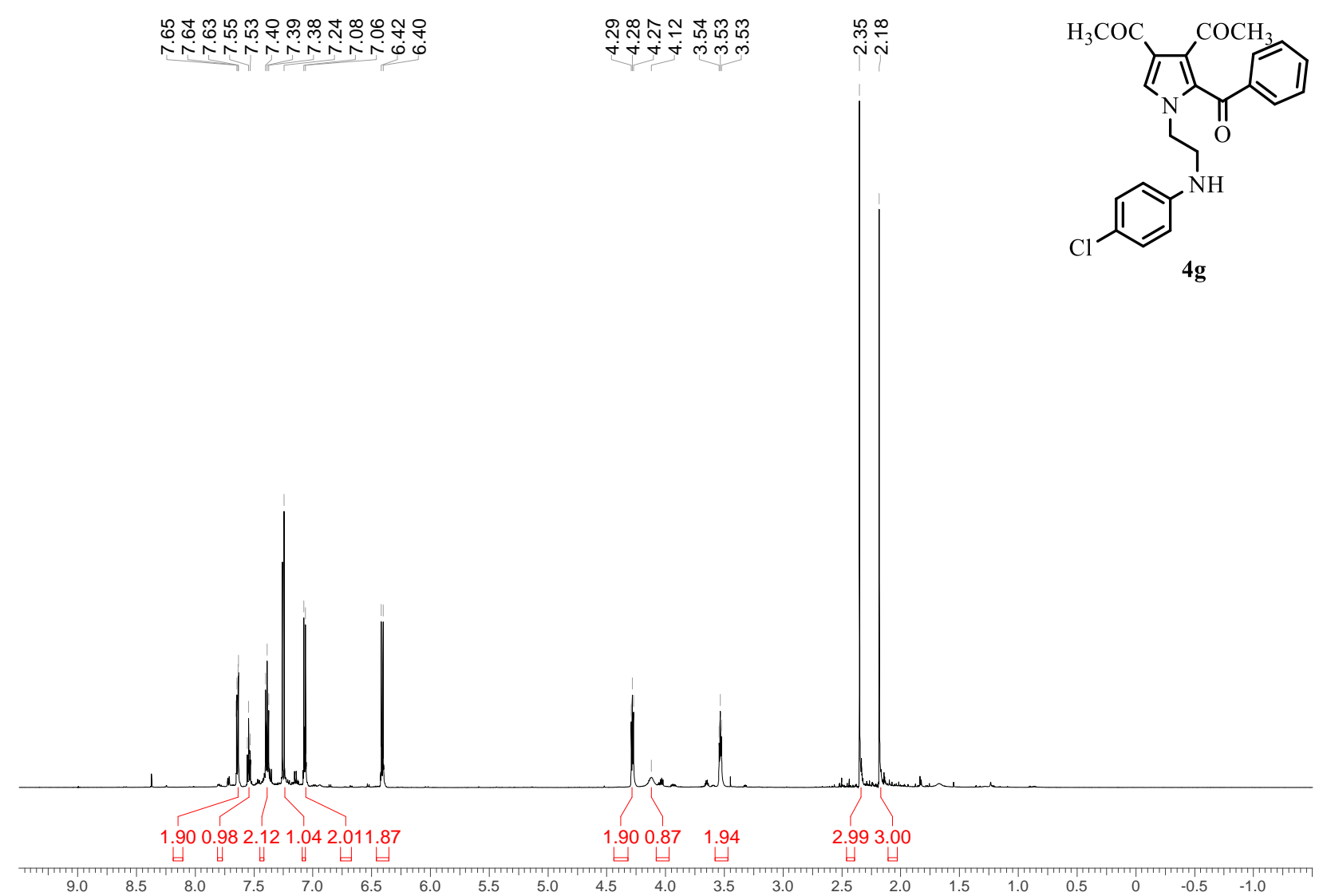

${ }^{13} \mathrm{C}$ NMR spectrum, $\mathrm{CDCl}_{3}, 150 \mathrm{MHz}$

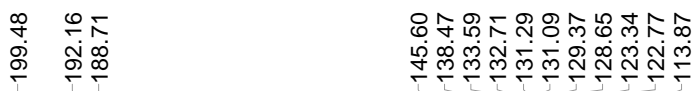

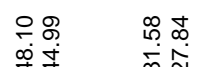

$\mathrm{CDCl} 3$
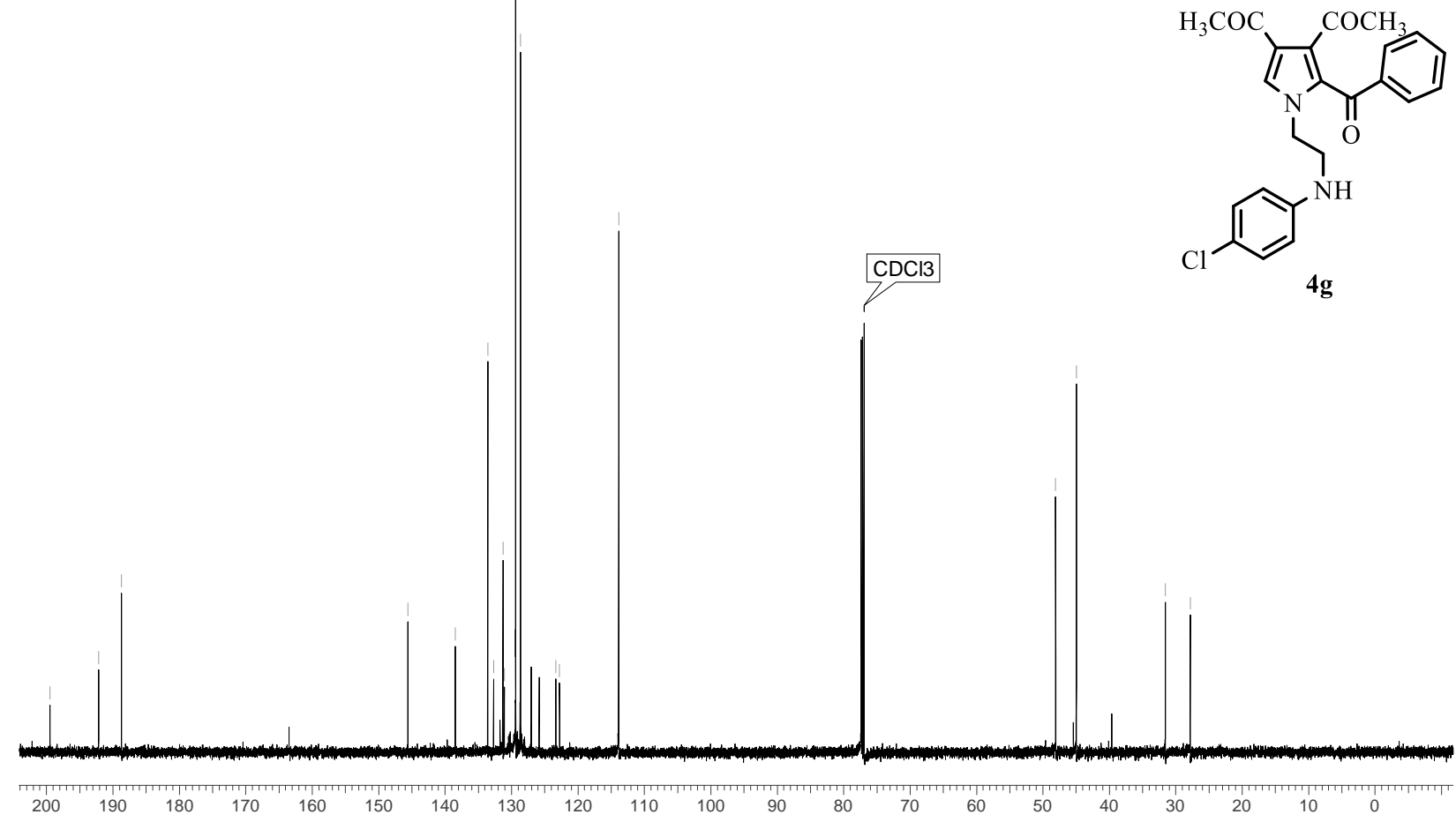
${ }^{1} \mathrm{H}$ NMR spectrum, $\mathrm{CDCl}_{3}, 600 \mathrm{MHZ}$

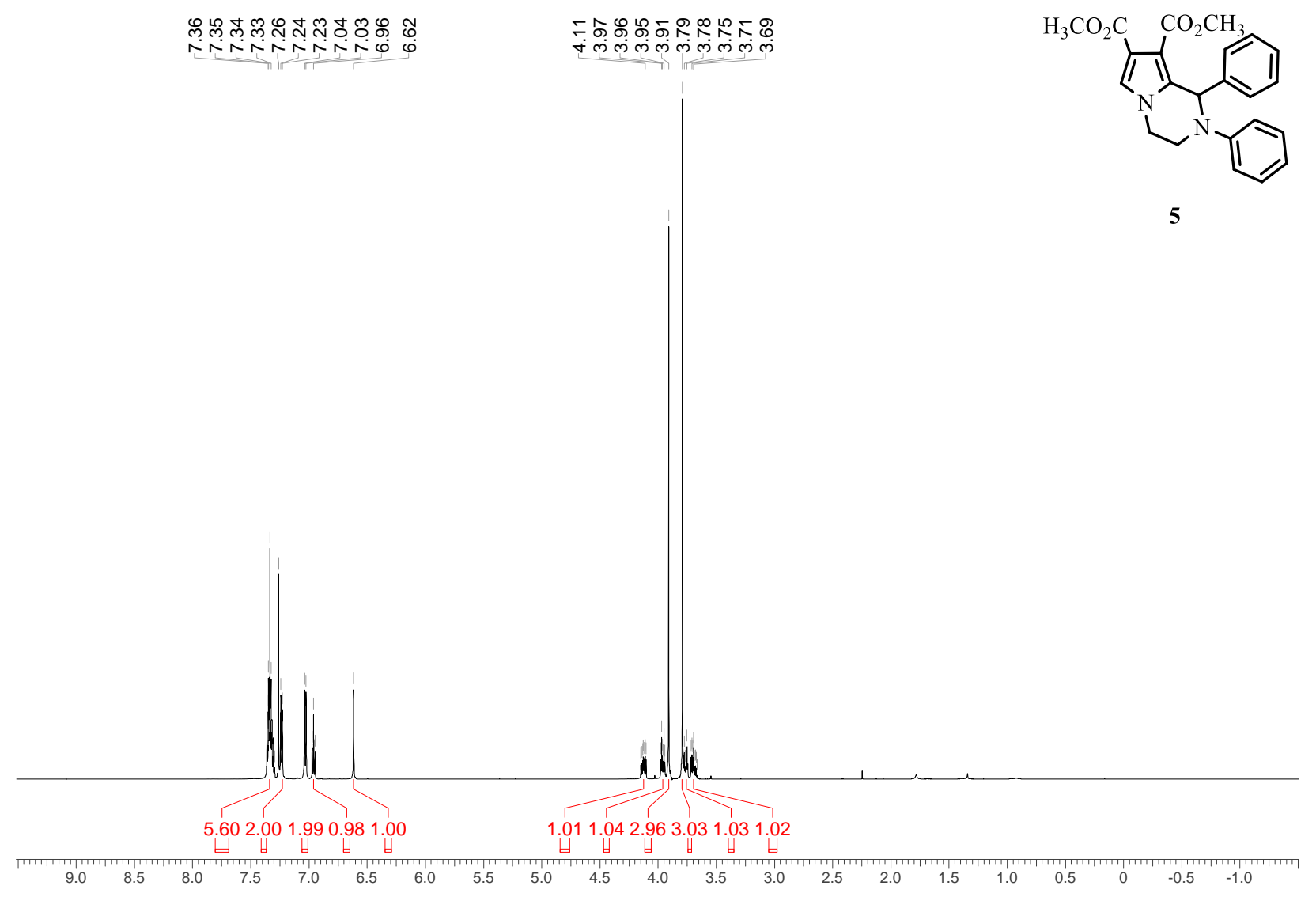

${ }^{13} \mathrm{C}$ NMR spectrum, $\mathrm{CDCl}_{3}, 150 \mathrm{MHz}$

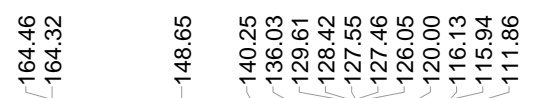

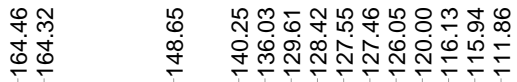

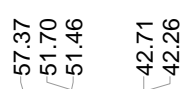

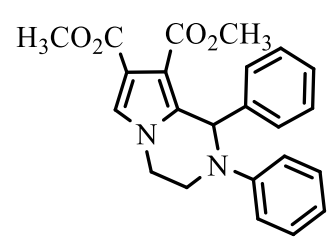

5

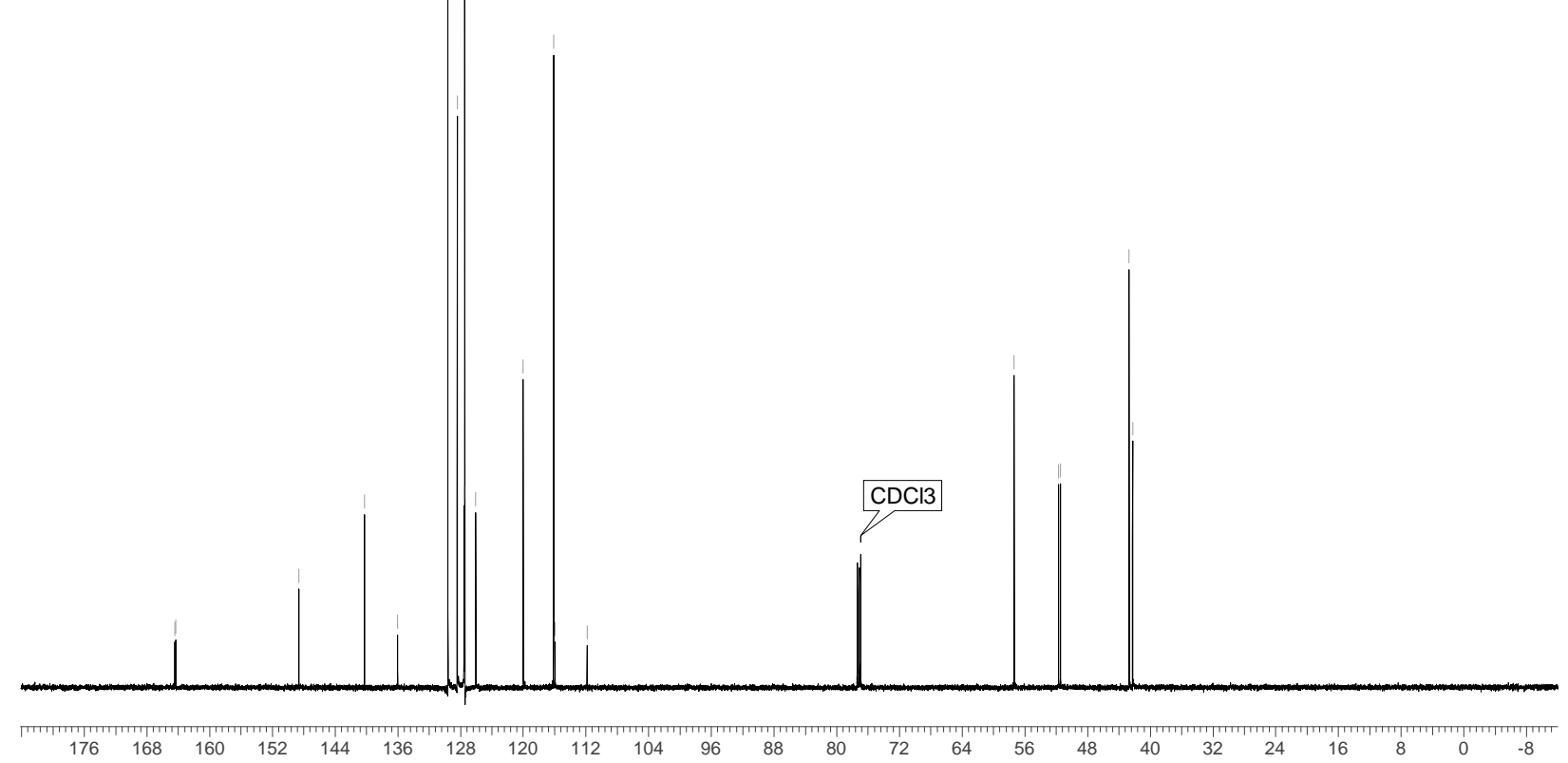


${ }^{1} \mathrm{H}$ NMR spectrum, $\mathrm{C}_{6} \mathrm{D}_{6}, 600 \mathrm{MHZ}$

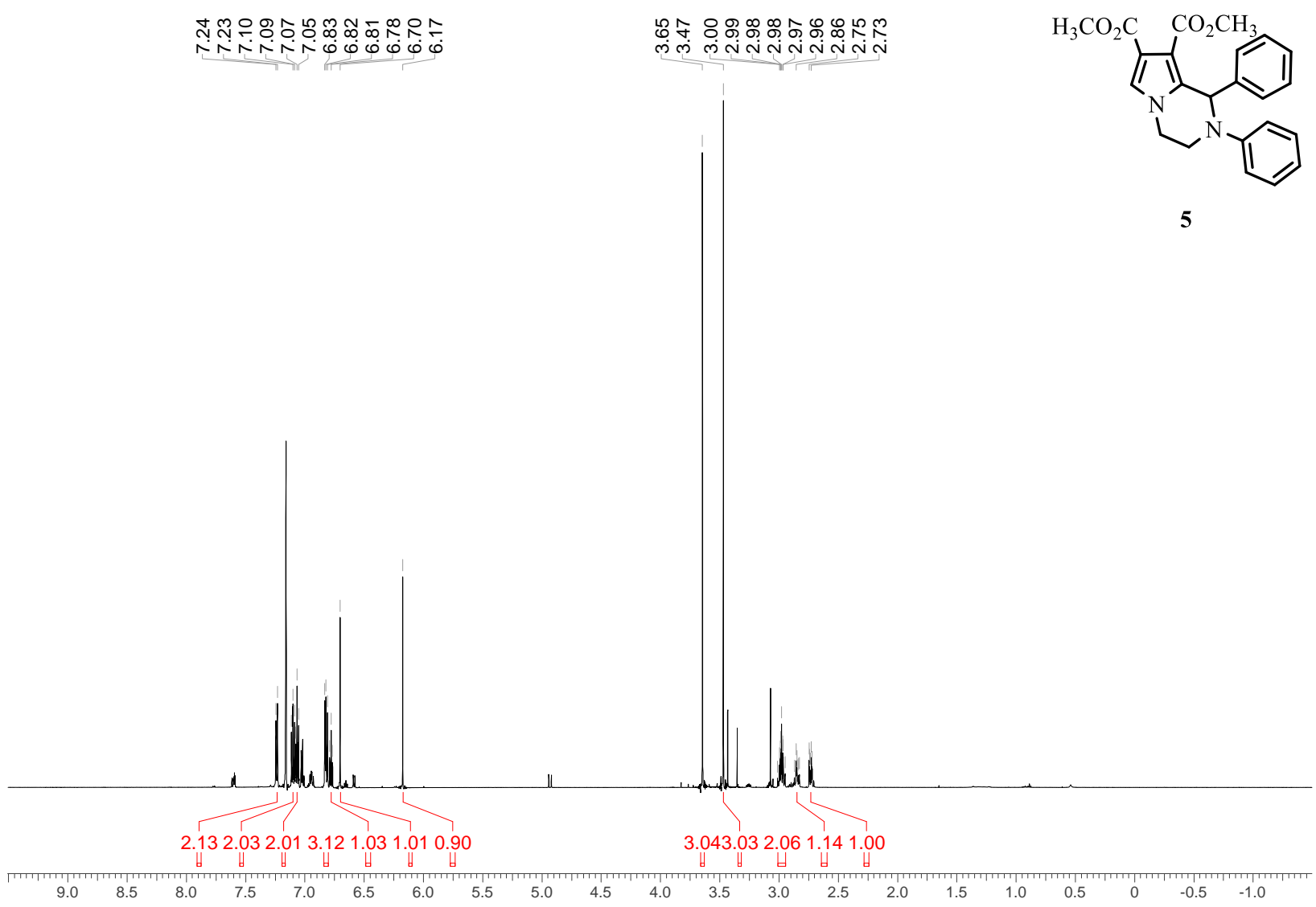

${ }^{13} \mathrm{C}$ NMR spectrum, $\mathrm{C}_{6} \mathrm{D}_{6}, 150 \mathrm{MHz}$

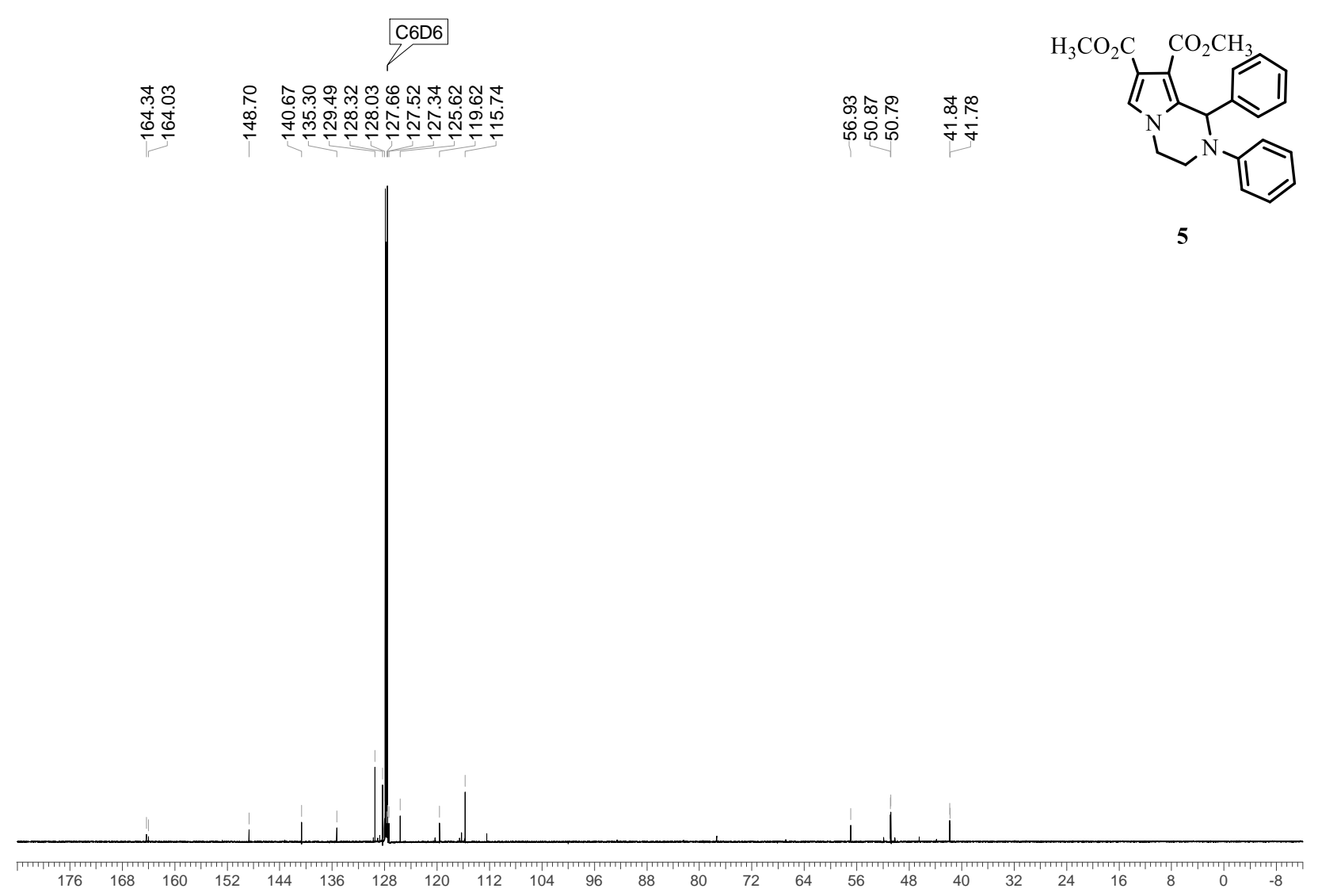


${ }^{1} \mathrm{H}$ NMR spectrum, $\mathrm{CDCl}_{3}, 600 \mathrm{MHZ}$

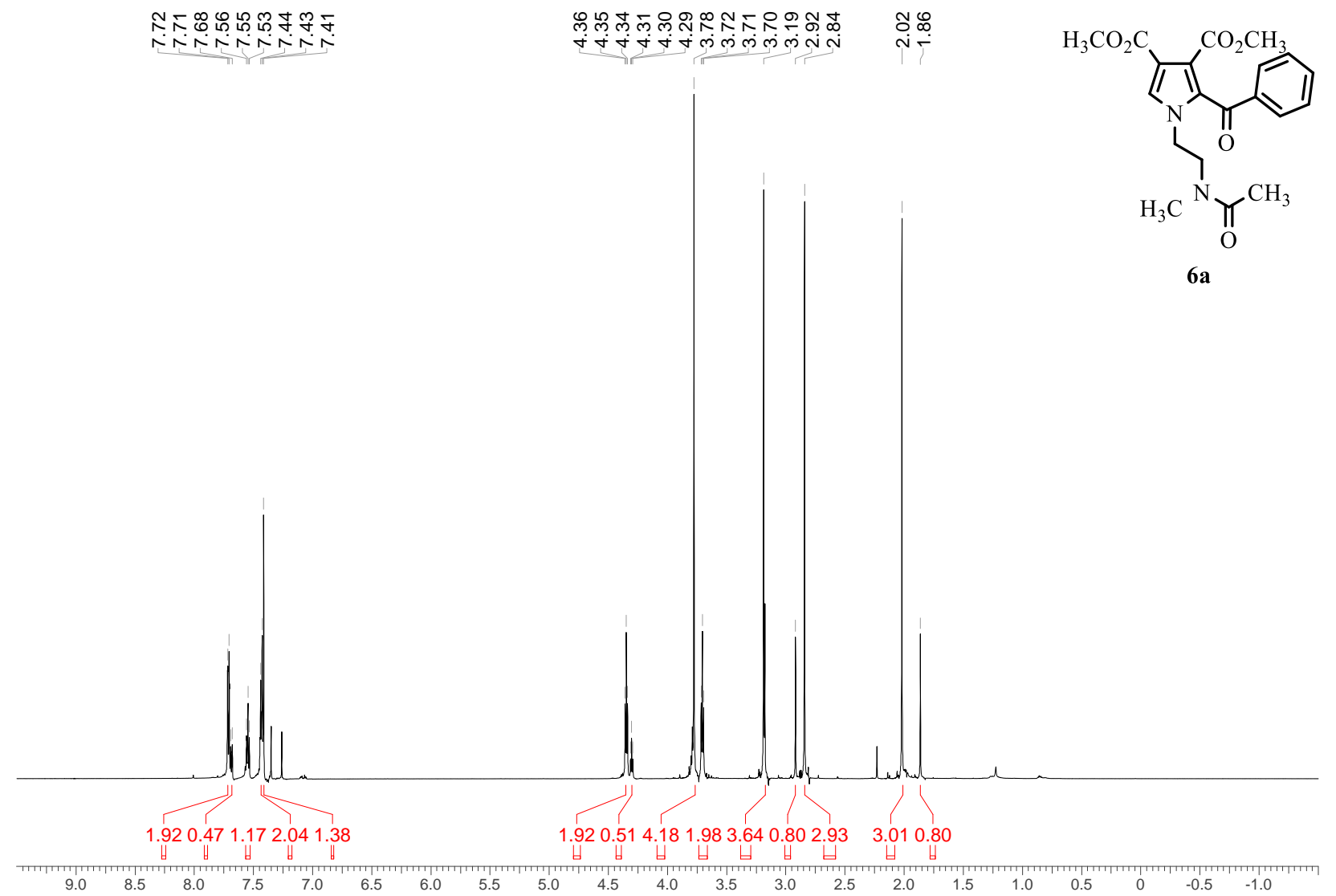

${ }^{13} \mathrm{C}$ NMR spectrum, $\mathrm{CDCl}_{3}, 150 \mathrm{MHz}$

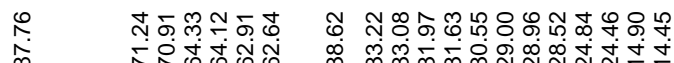

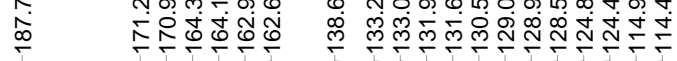

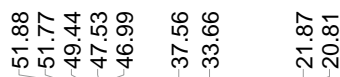

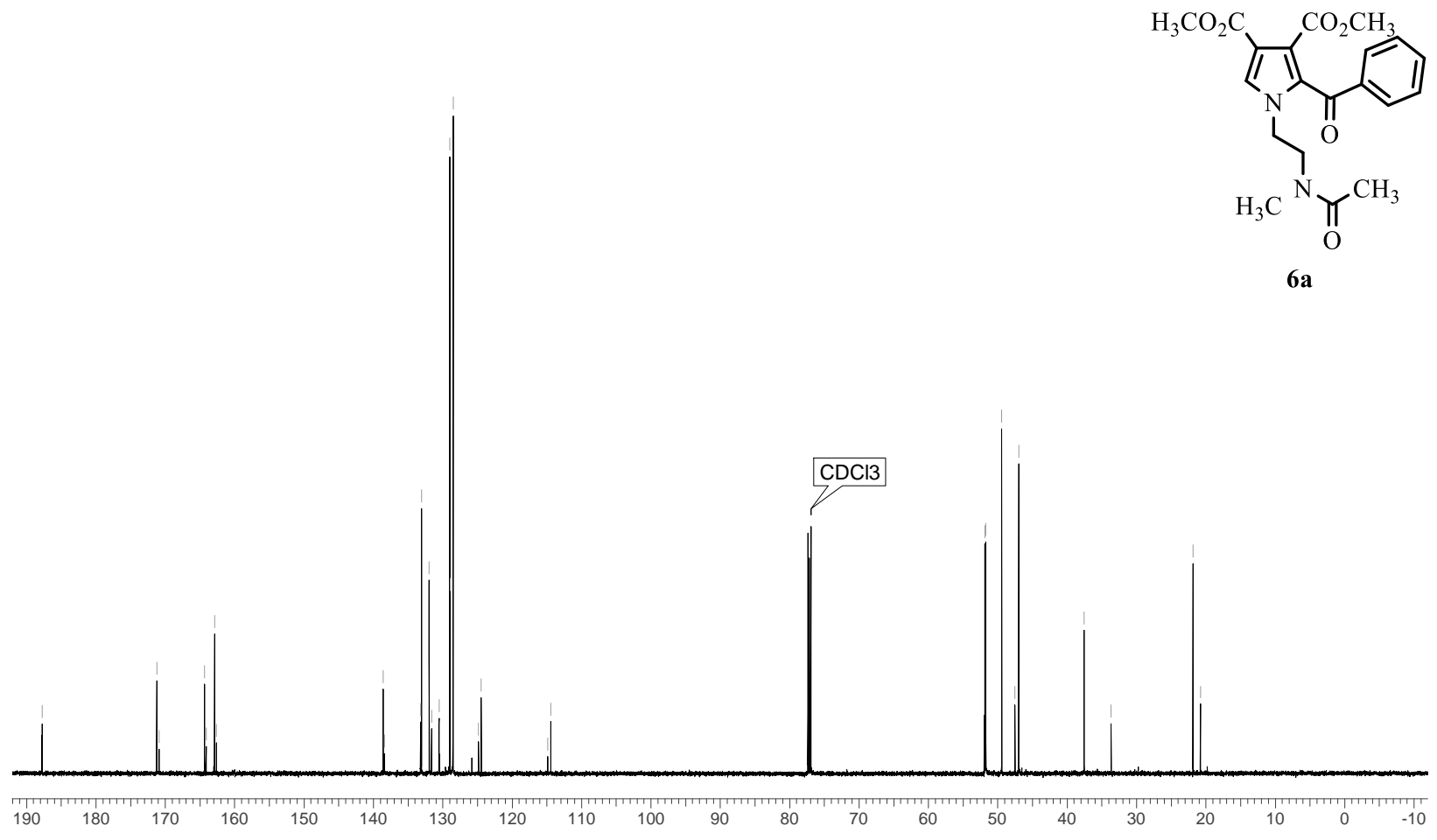


${ }^{1} \mathrm{H}$ NMR spectrum, $\mathrm{CDCl}_{3}, 600 \mathrm{MHZ}$

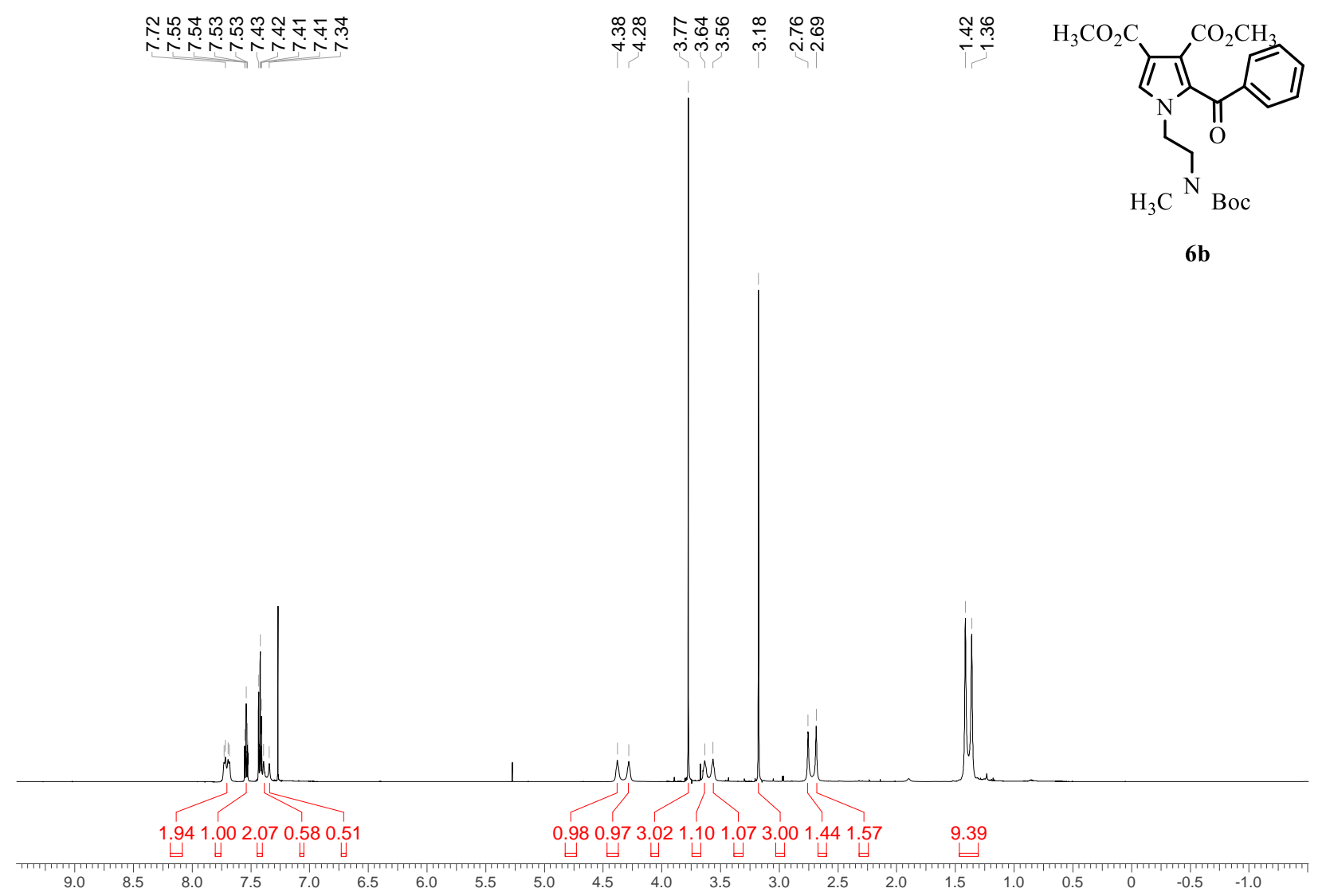

${ }^{13} \mathrm{C}$ NMR spectrum, $\mathrm{CDCl}_{3}, 150 \mathrm{MHz}$

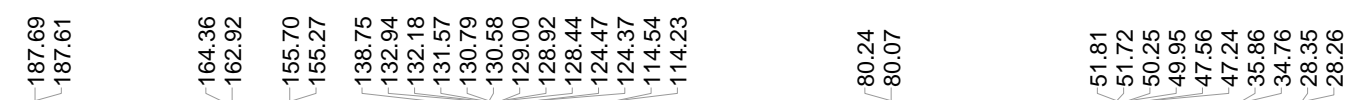

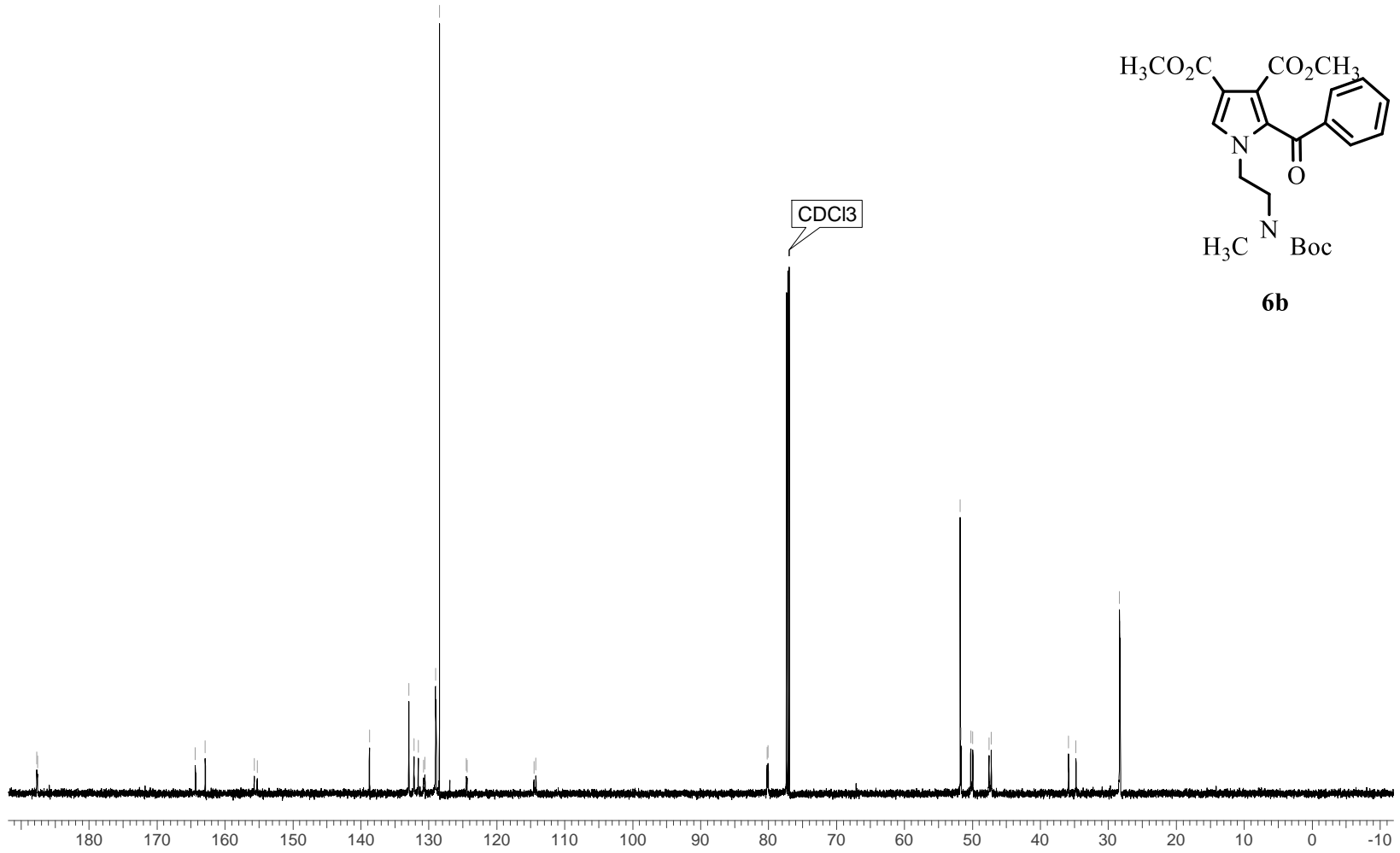


${ }^{1} \mathrm{H}$ NMR spectrum, $\mathrm{CDCl}_{3}, 600 \mathrm{MHZ}$

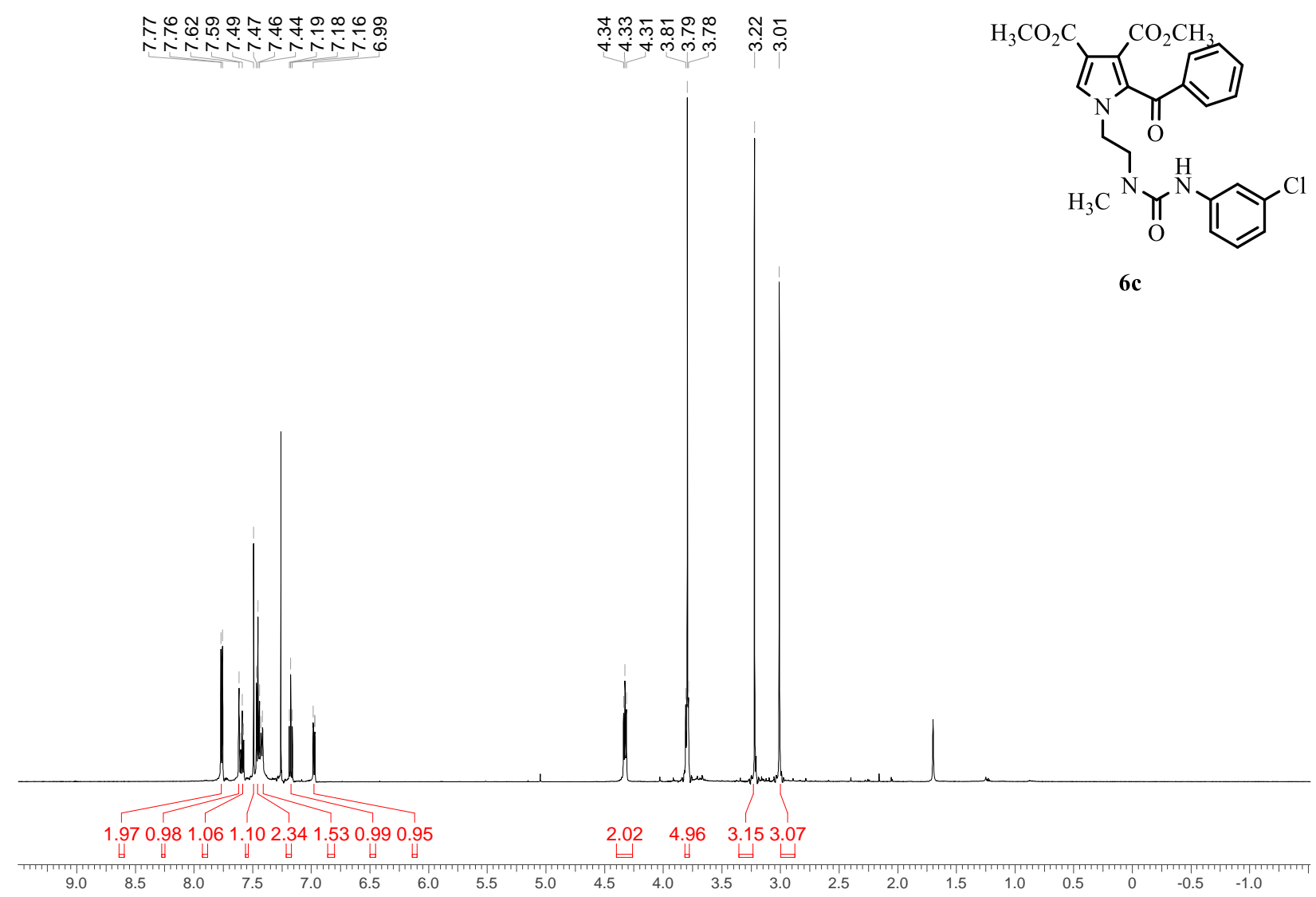

${ }^{13} \mathrm{C}$ NMR spectrum, $\mathrm{CDCl}_{3}, 150 \mathrm{MHz}$

की

車

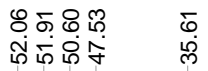

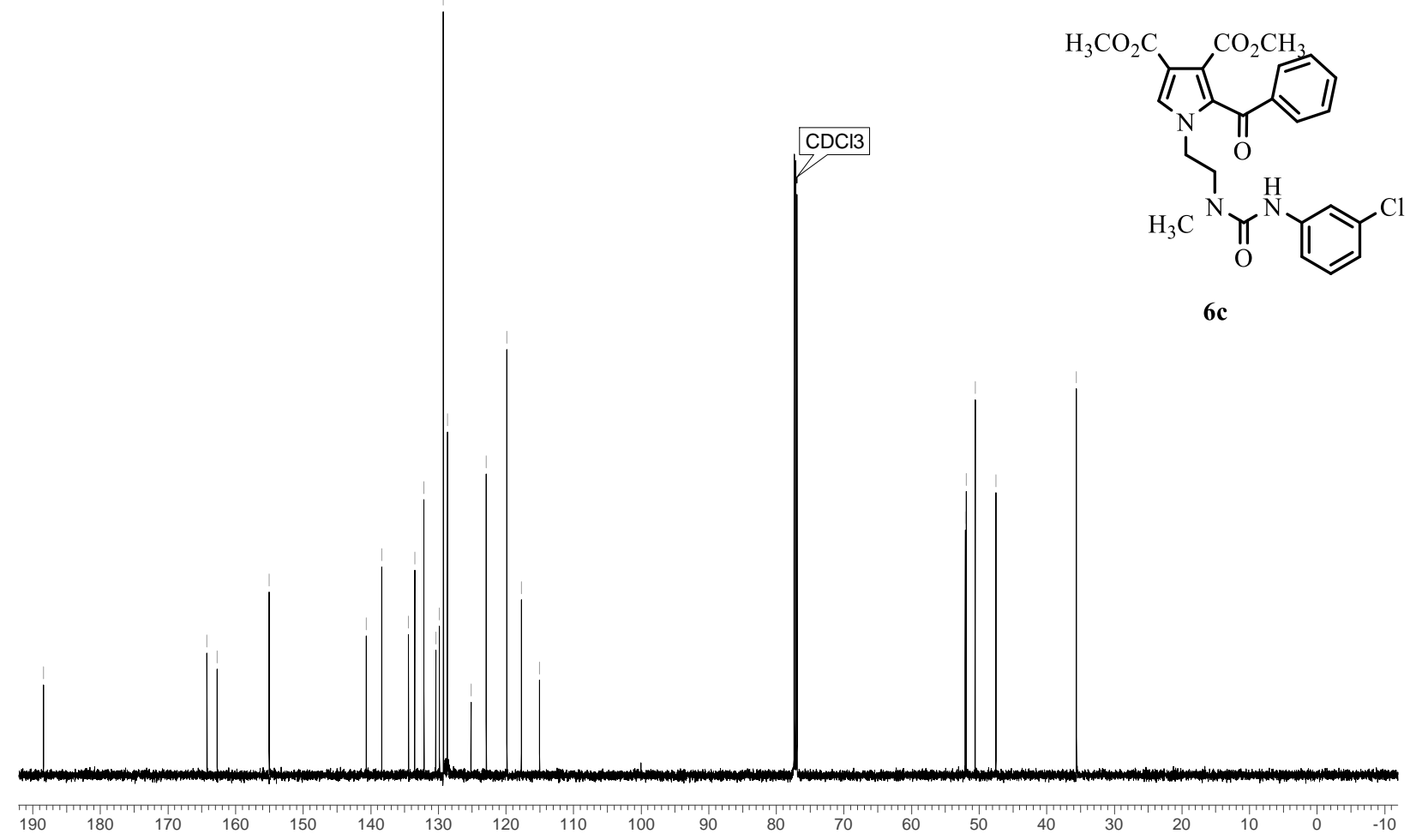


${ }^{1} \mathrm{H}$ NMR spectrum, $\mathrm{CDCl}_{3}, 600 \mathrm{MHZ}$

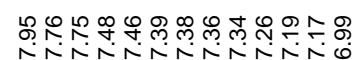

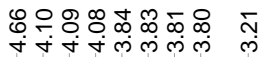

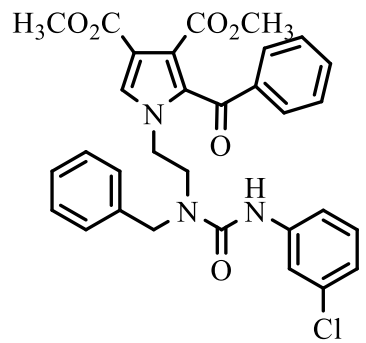

6d

$1.95 \quad 1.962 .063 .022 .98$

0.972 .981 .161 .012 .122 .193 .050 .871 .080 .97 $\forall \forall \forall \forall \forall$

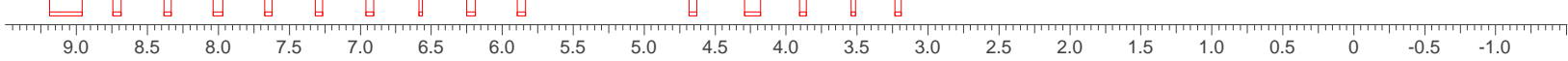

${ }^{13} \mathrm{C}$ NMR spectrum, $\mathrm{CDCl}_{3}, 150 \mathrm{MHz}$

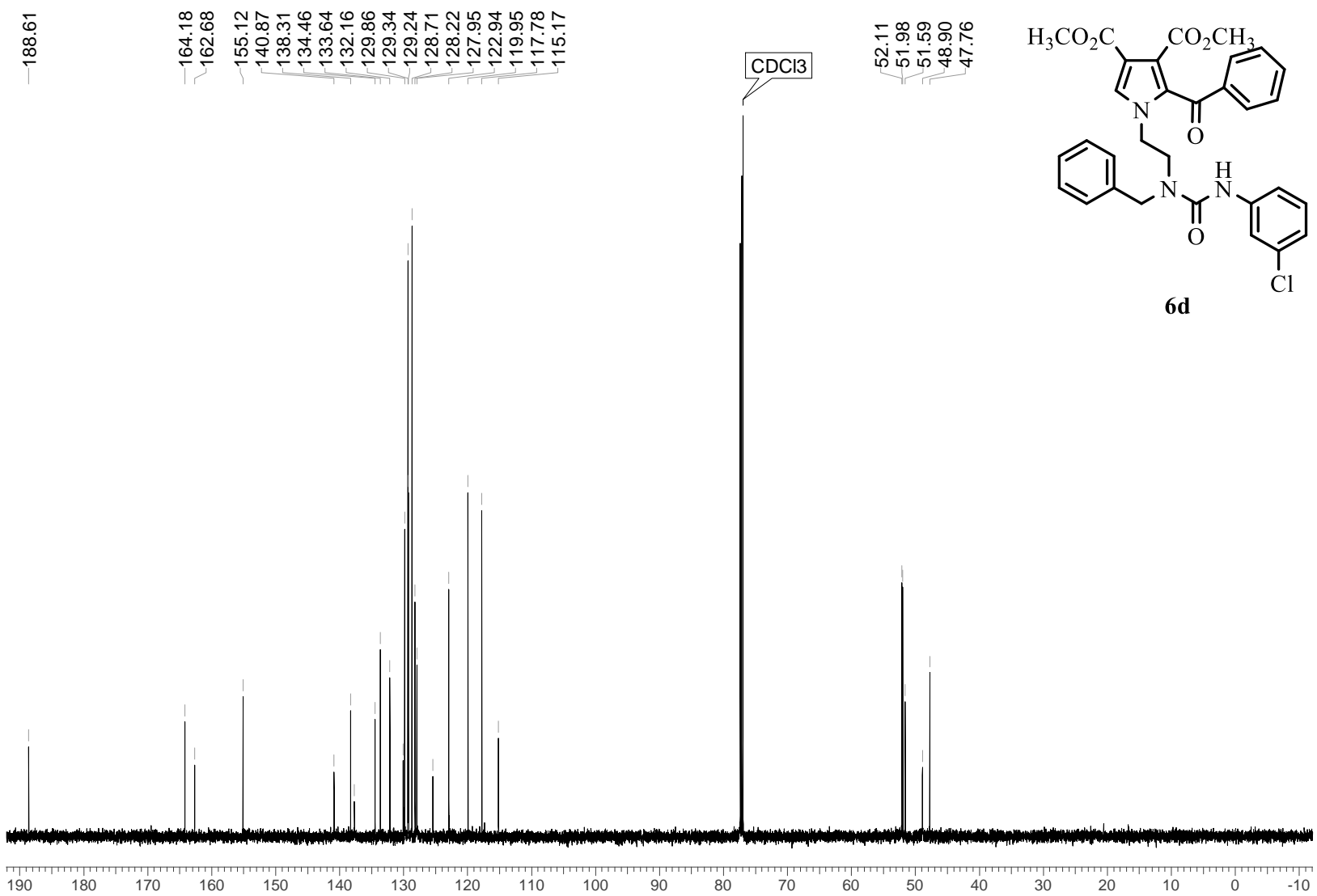


${ }^{1} \mathrm{H}$ NMR spectrum, $\mathrm{CDCl}_{3}, 600 \mathrm{MHZ}$

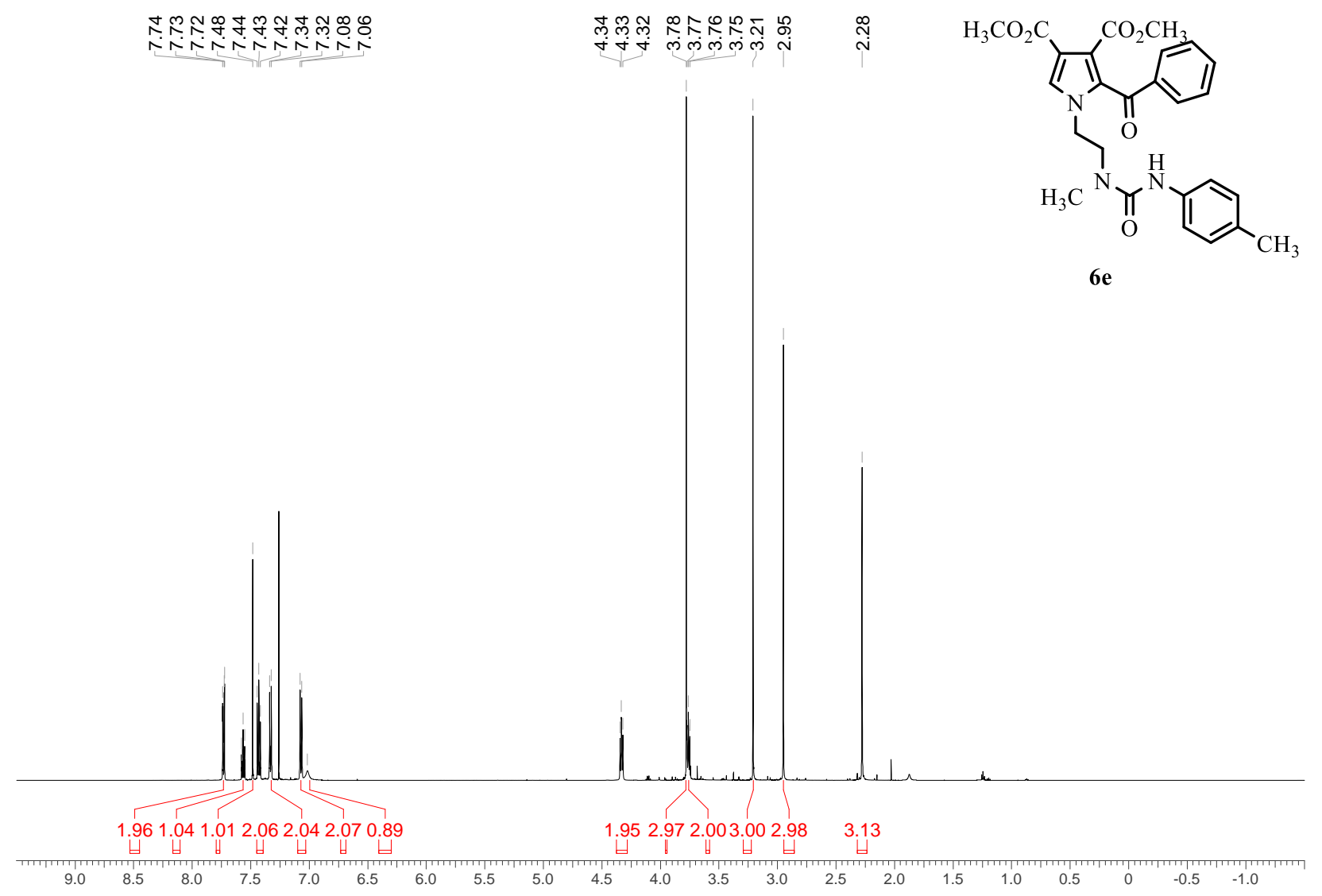

${ }^{13} \mathrm{C}$ NMR spectrum, $\mathrm{CDCl}_{3}, 150 \mathrm{MHz}$

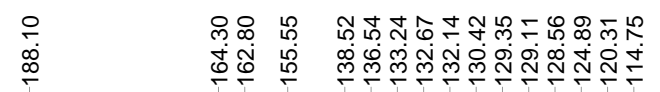

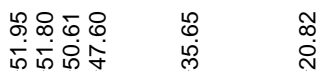

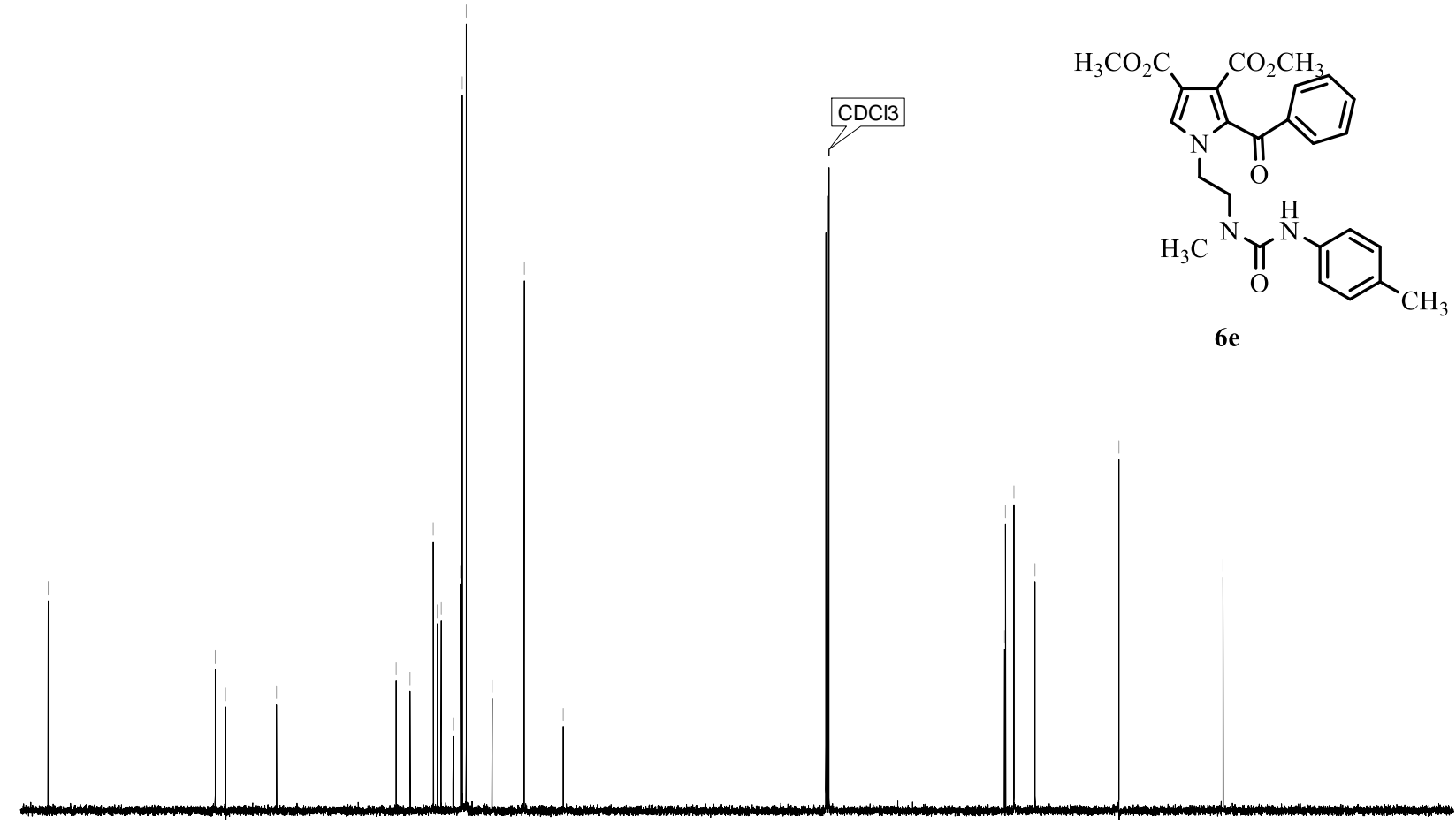

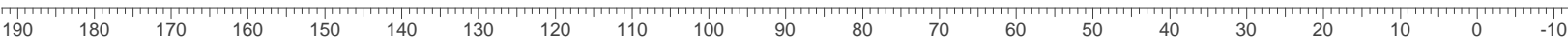


${ }^{1} \mathrm{H}$ NMR spectrum, DMSO-d 6 , 600 MHZ

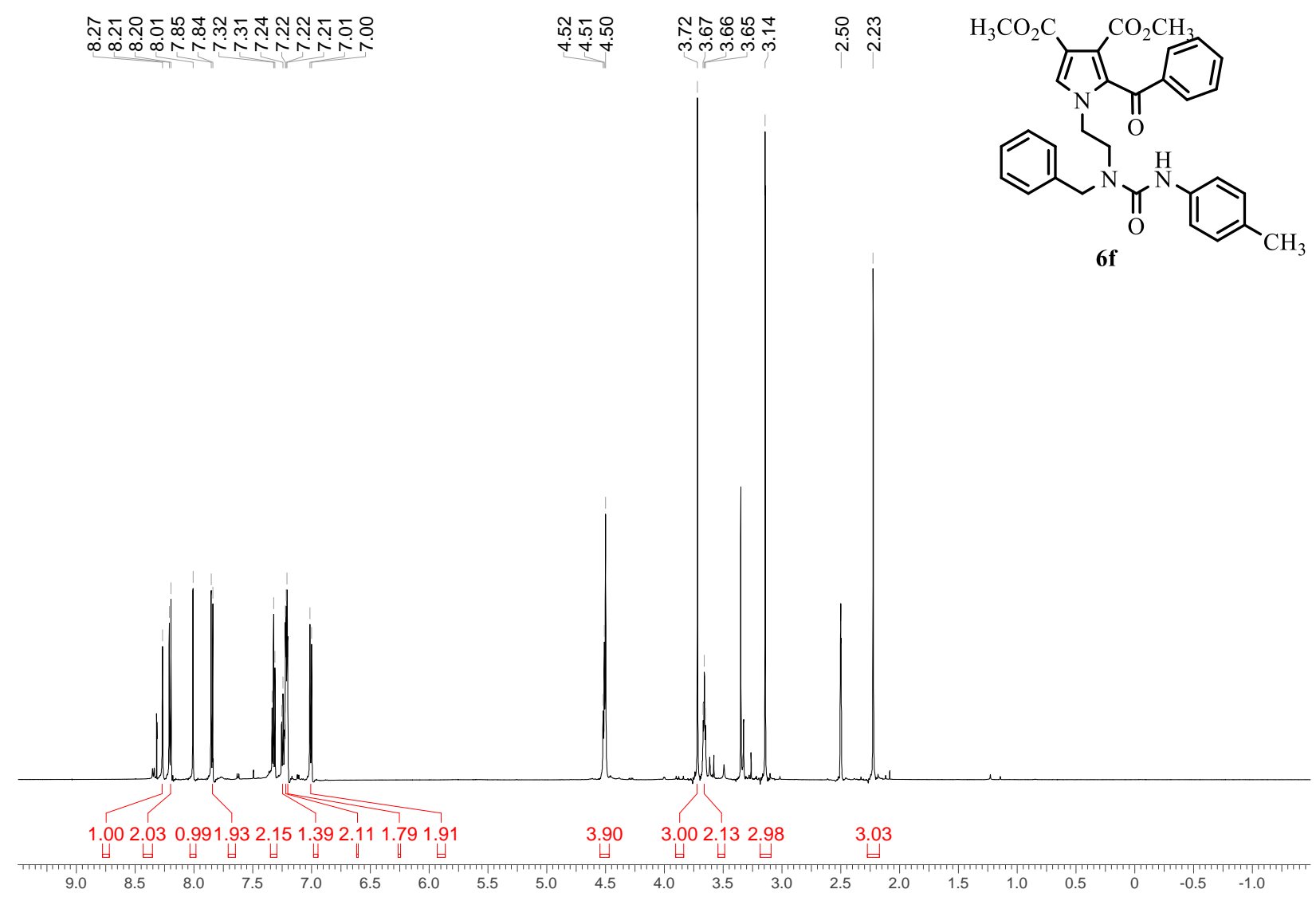

${ }^{13} \mathrm{C}$ NMR spectrum, DMSO-d $6,150 \mathrm{MHz}$

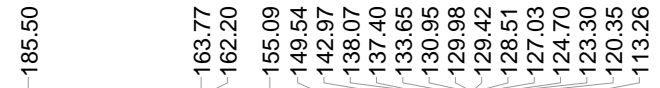

ญำ

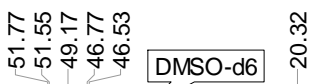
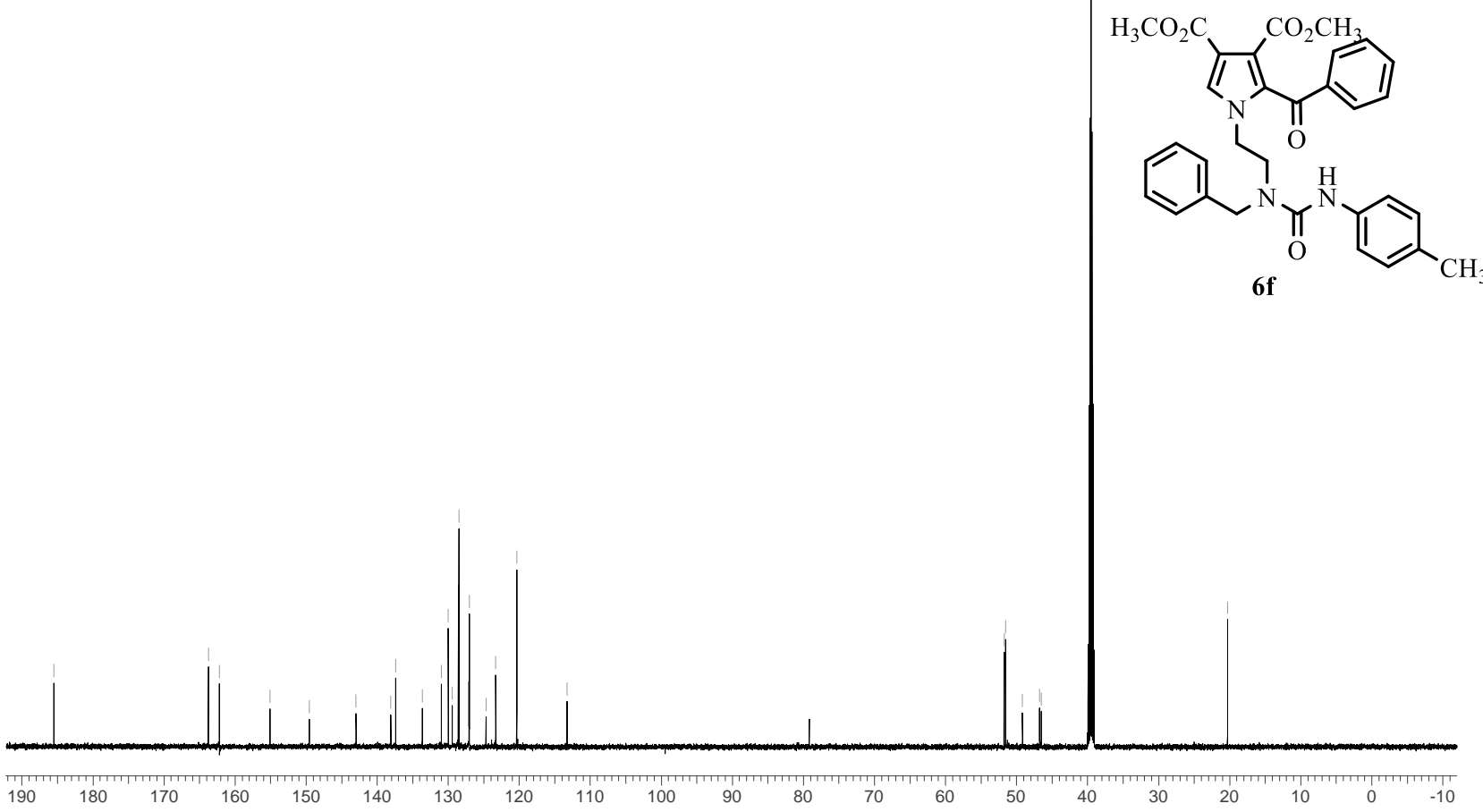
${ }^{1} \mathrm{H}$ NMR spectrum, $\mathrm{CDCl}_{3}, 600 \mathrm{MHZ}$

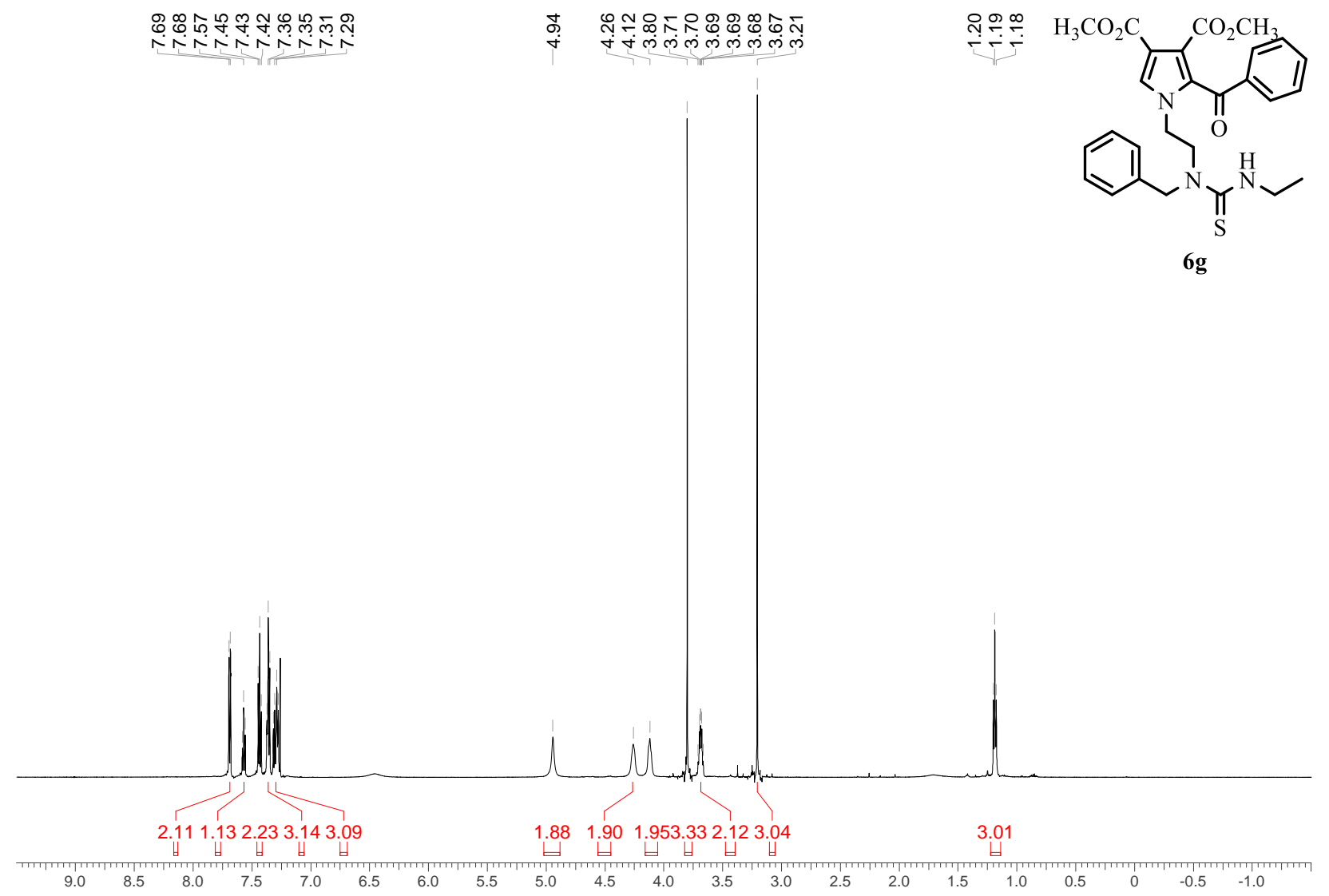

${ }^{13} \mathrm{C}$ NMR spectrum, $\mathrm{CDCl}_{3}, 150 \mathrm{MHz}$

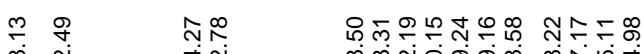

要

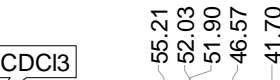

$\stackrel{\bar{m}}{\dot{j}}$

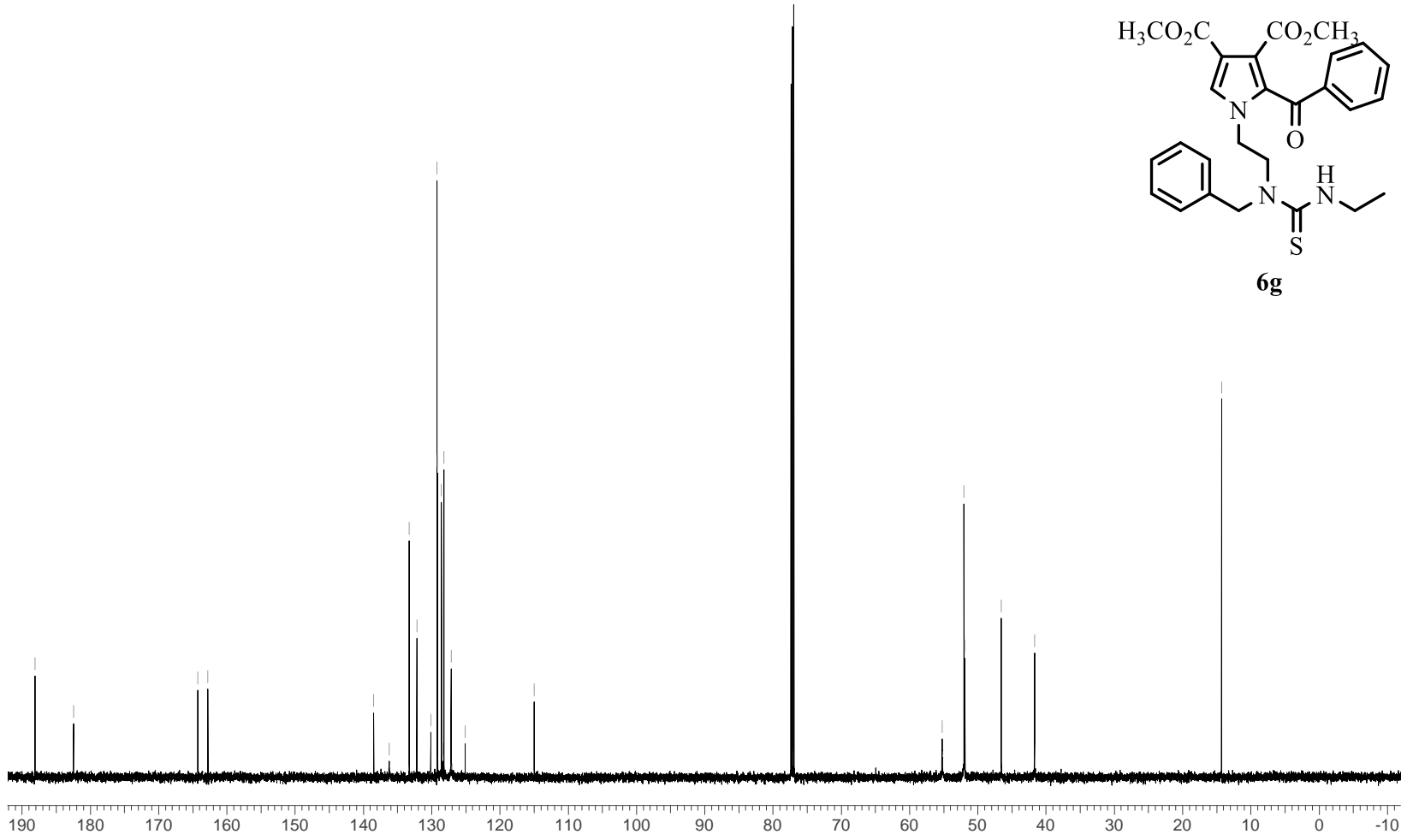


${ }^{1} \mathrm{H}$ NMR spectrum, $\mathrm{CDCl}_{3}, 600 \mathrm{MHZ}$

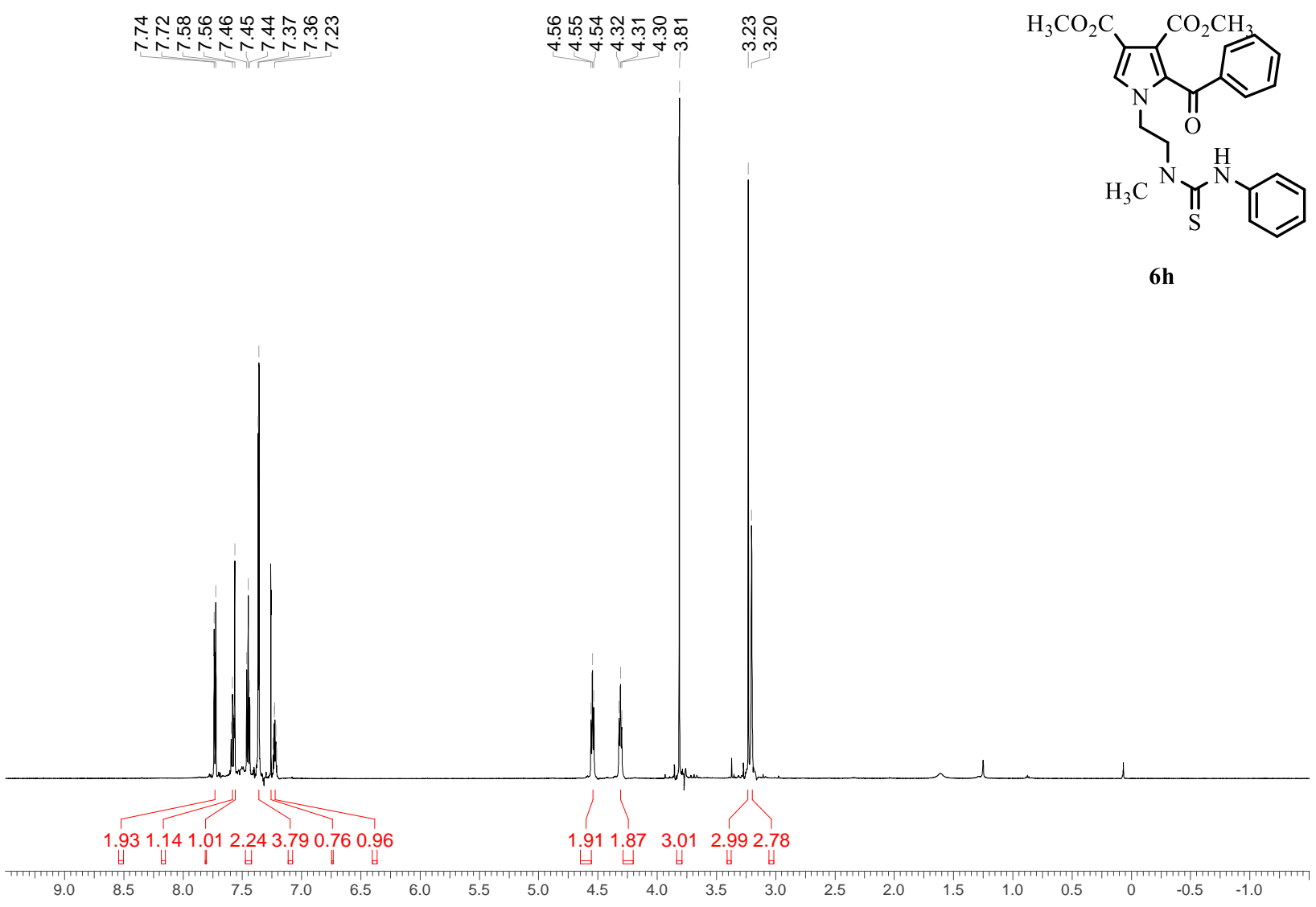

${ }^{13} \mathrm{C}$ NMR spectrum, $\mathrm{CDCl}_{3}, 150 \mathrm{MHz}$

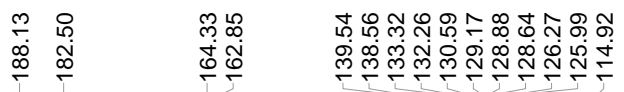

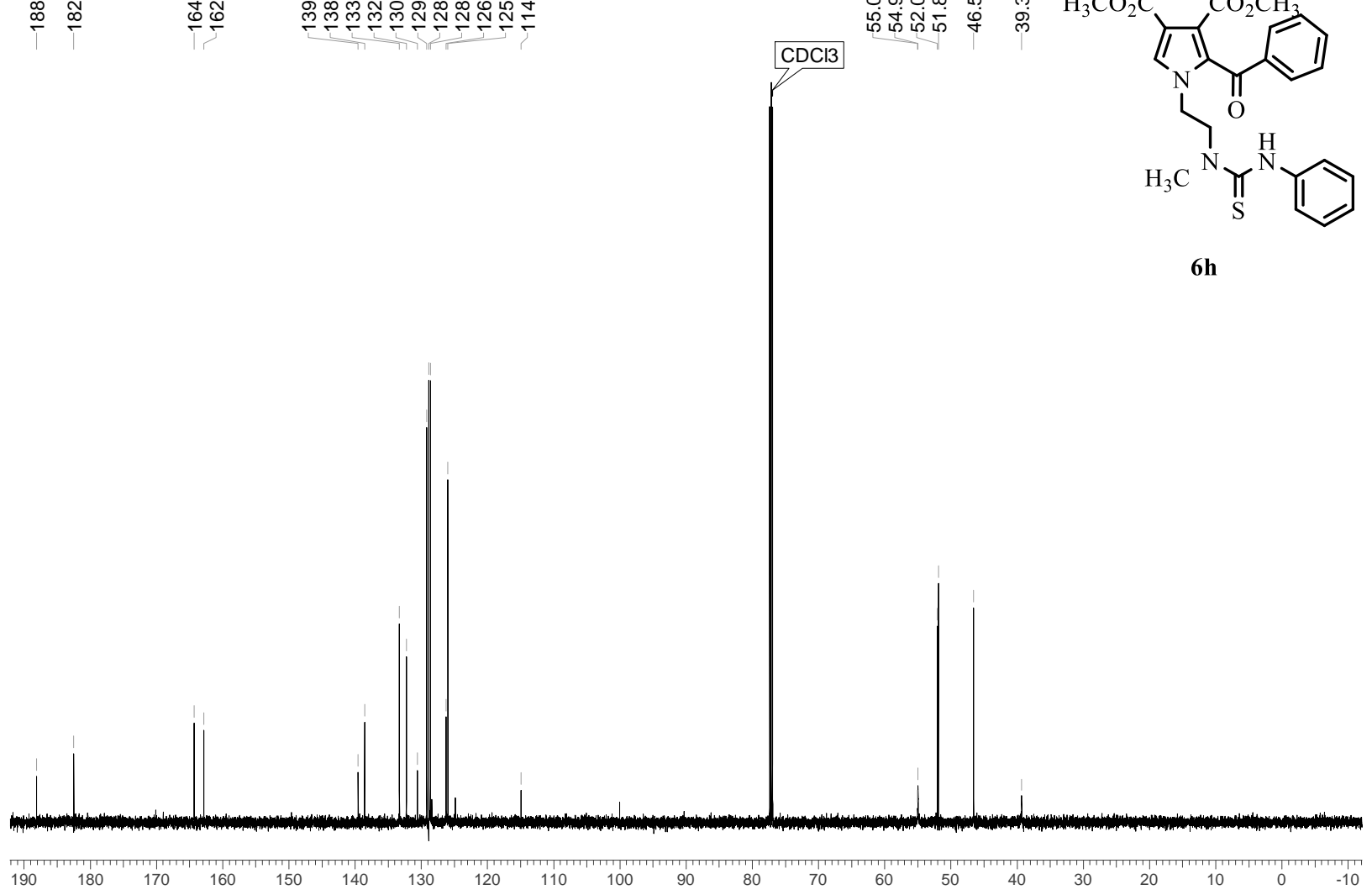


${ }^{1} \mathrm{H}$ NMR spectrum, $\mathrm{CDCl}_{3}, 600 \mathrm{MHZ}$

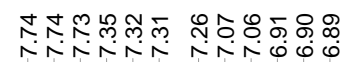

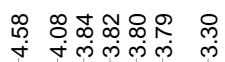

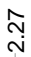

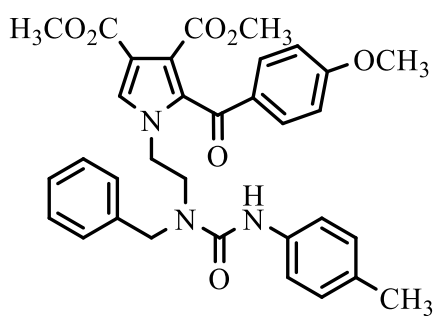

$6 \mathbf{i}$

2.011 .112 .312 .343 .151 .412 .141 .99

0.002 .001 .983 .155 .143 .00

3.29

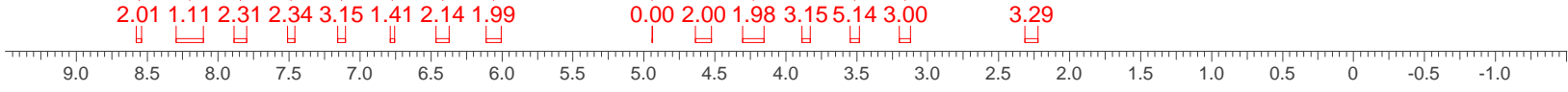

${ }^{13} \mathrm{C}$ NMR spectrum, $\mathrm{CDCl}_{3}, 150 \mathrm{MHz}$

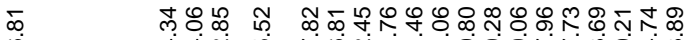

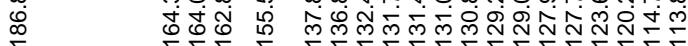

함ำ

$\stackrel{\infty}{\infty}$

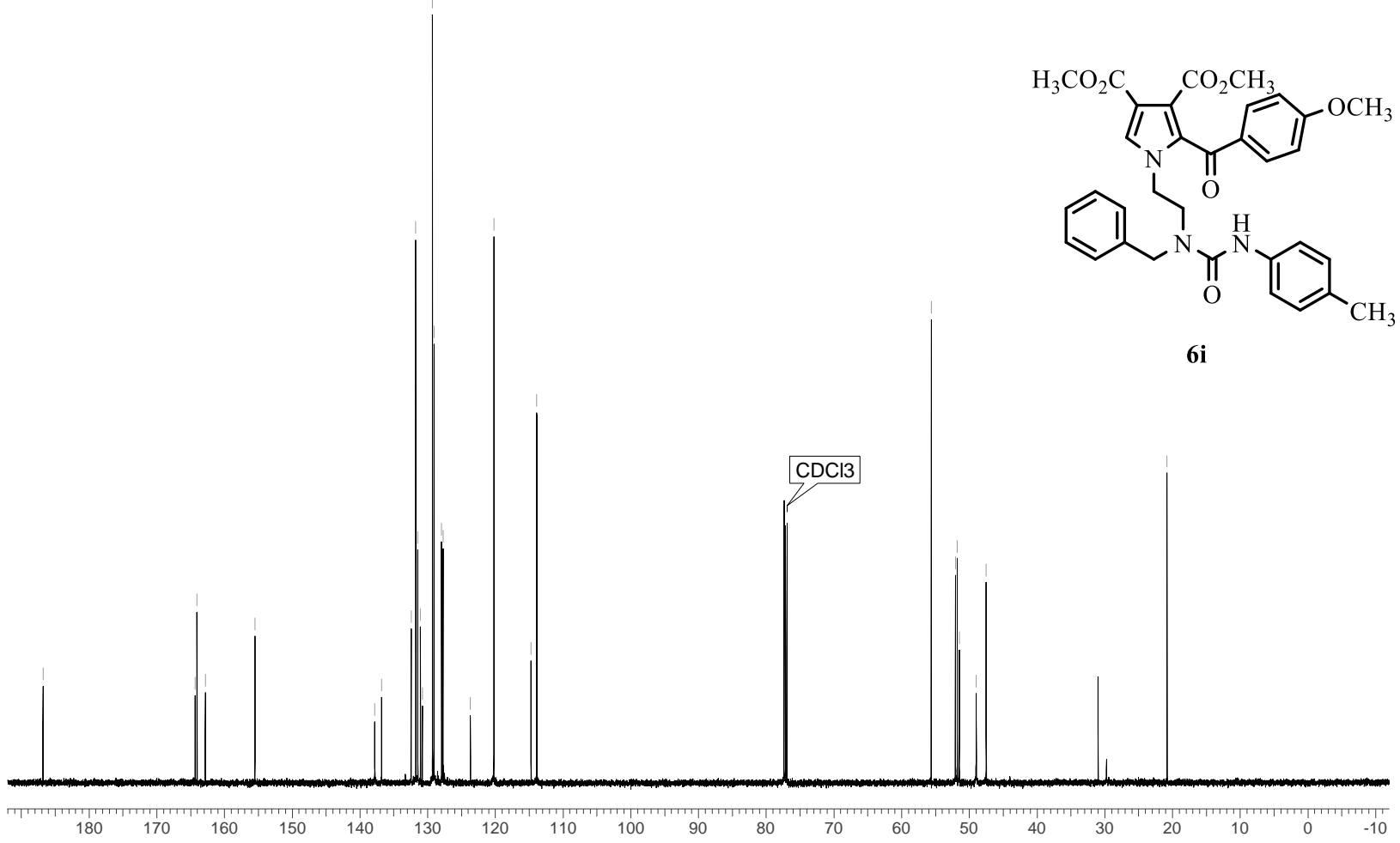


${ }^{1} \mathrm{H}$ NMR spectrum, $\mathrm{CDCl}_{3}, 600 \mathrm{MHZ}$

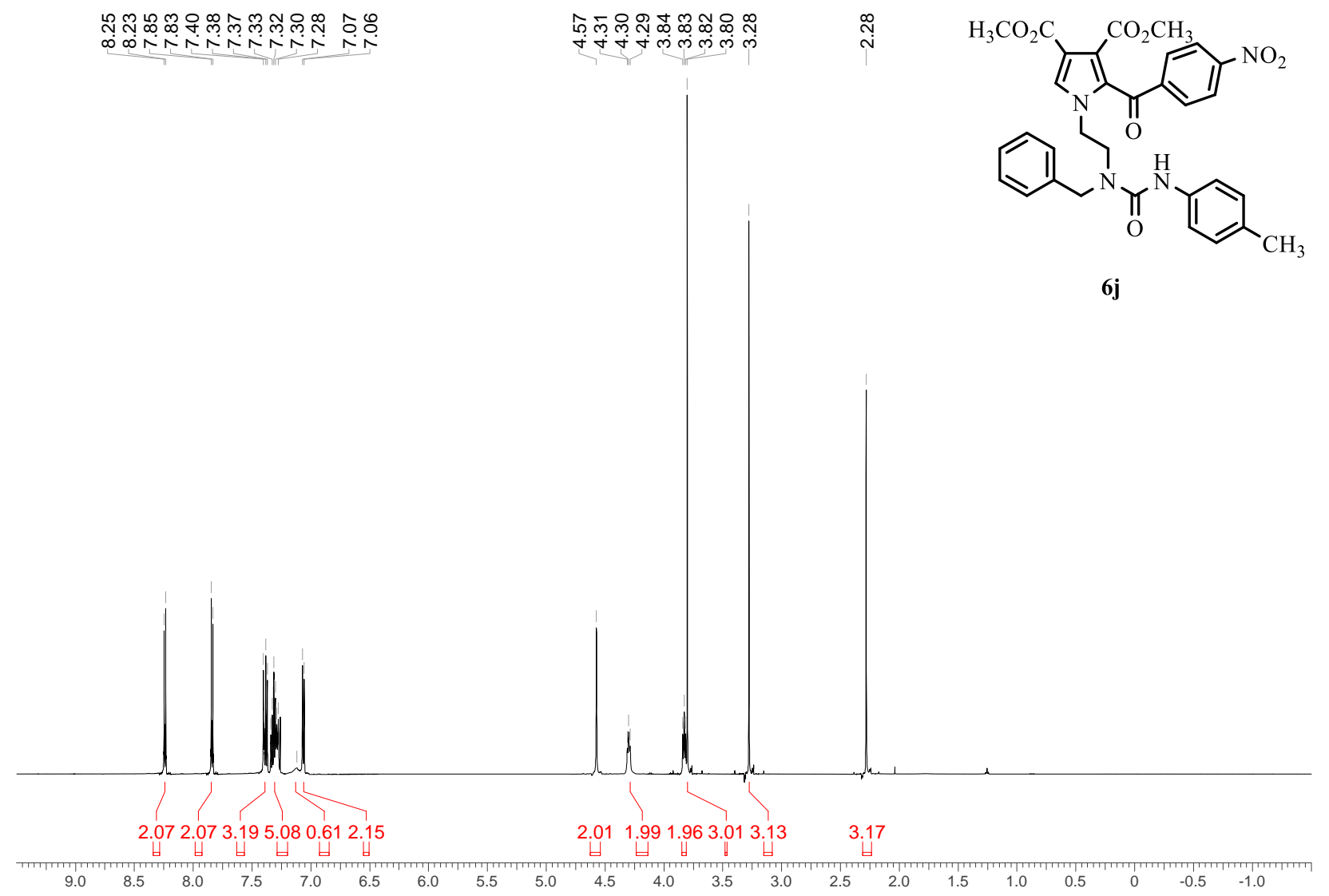

${ }^{13} \mathrm{C}$ NMR spectrum, $\mathrm{CDCl}_{3}, 150 \mathrm{MHz}$

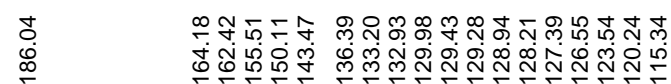

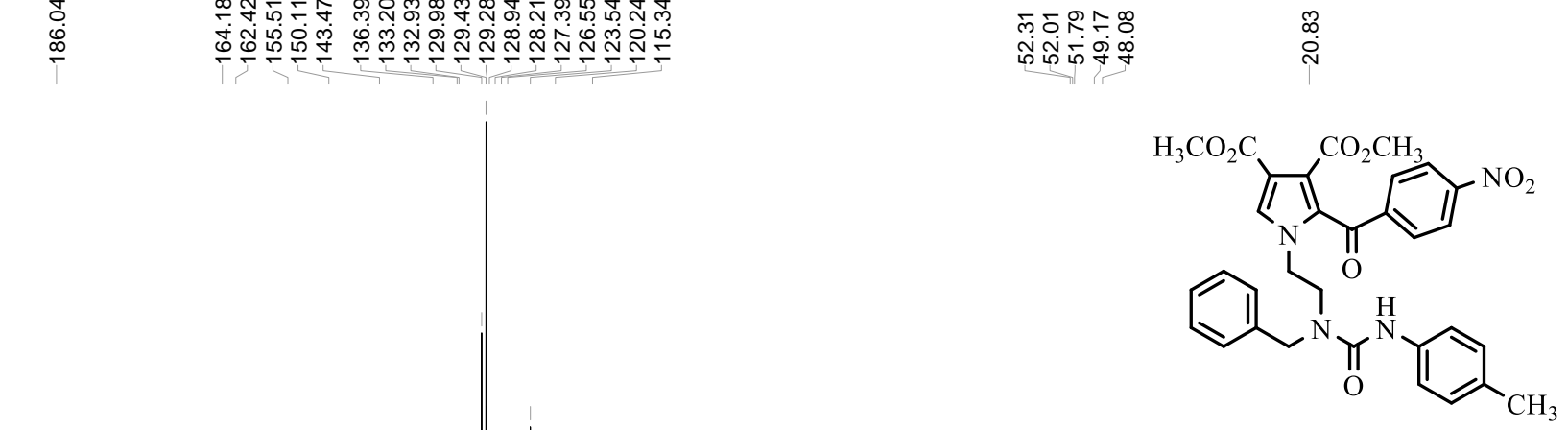

6j

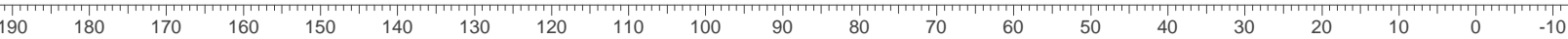


${ }^{1} \mathrm{H}$ NMR spectrum, $\mathrm{CDCl}_{3}, 600 \mathrm{MHZ}$

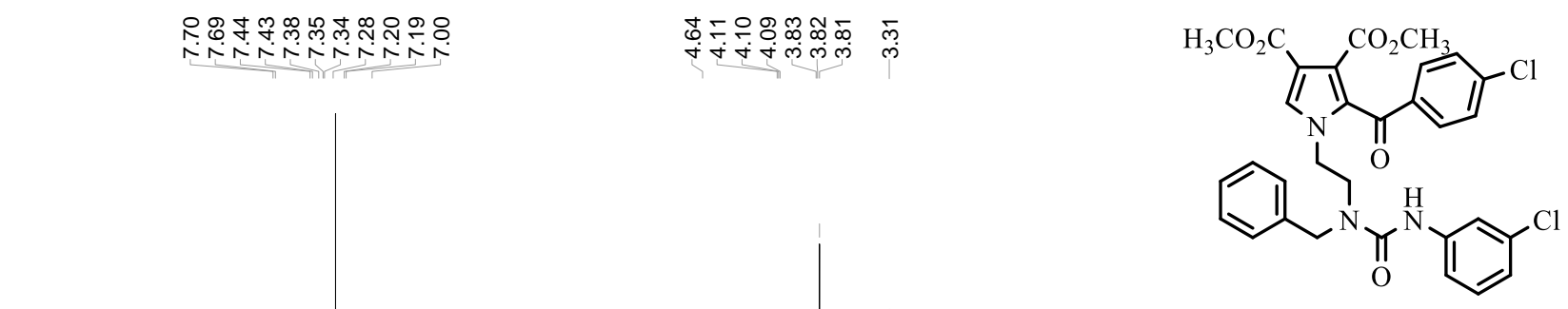

$6 \mathbf{k}$

2.811 .082 .062 .132 .963 .741 .060 .93

1.911 .871 .493 .352 .98

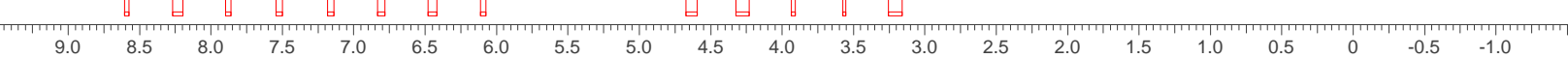

${ }^{13} \mathrm{C}$ NMR spectrum, $\mathrm{CDCl}_{3}, 150 \mathrm{MHz}$

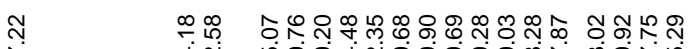

कon

$\mathrm{CDCl} 3$

लํํำ

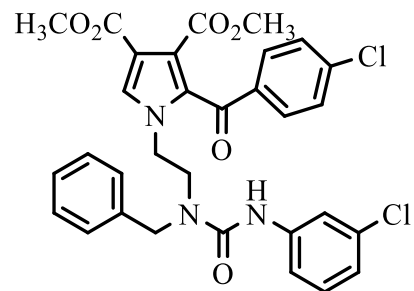

$6 \mathbf{k}$

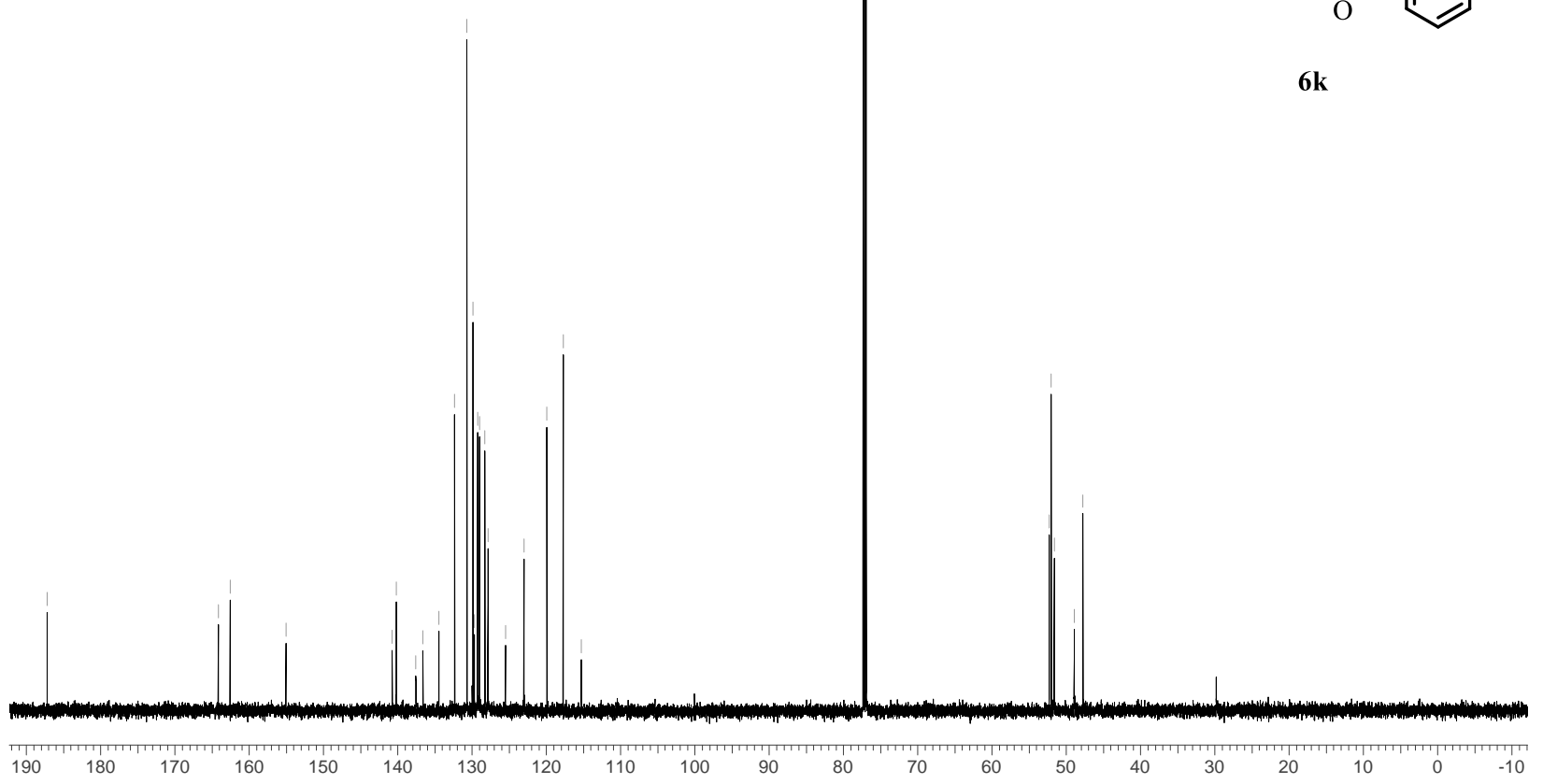


${ }^{1} \mathrm{H}$ NMR spectrum, $\mathrm{CDCl}_{3}, 600 \mathrm{MHZ}$

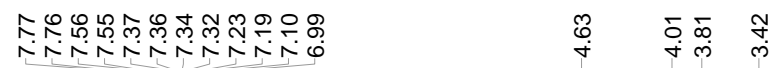

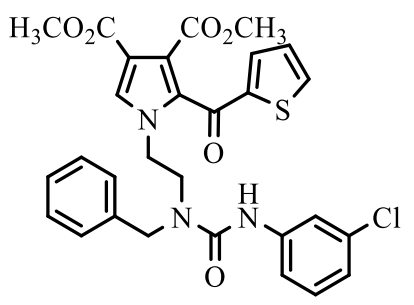

61

0.881 .130 .952 .022 .342 .871 .101 .121 .100 .93

$1.85 \quad 1.824 .803 .00$

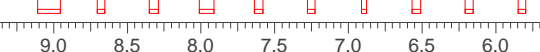 \\ ${ }^{13} \mathrm{C}$ NMR spectrum, $\mathrm{CDCl}_{3}, 150 \mathrm{MHz}$

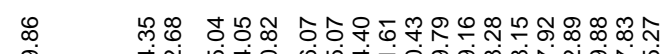

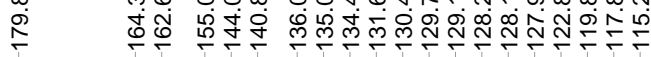

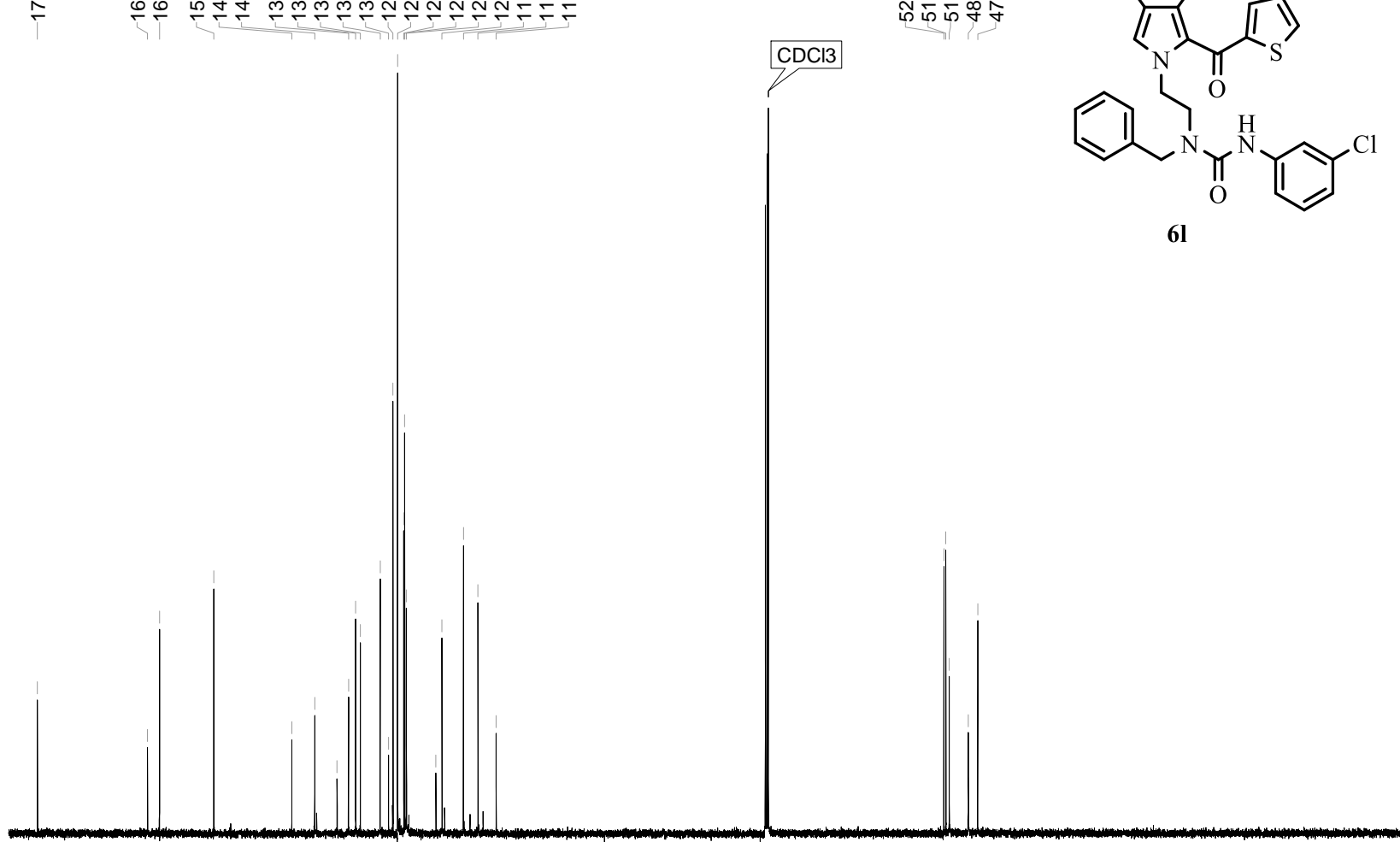

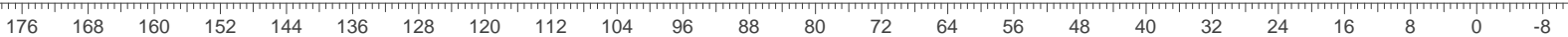


${ }^{1} \mathrm{H}$ NMR spectrum, $\mathrm{CDCl}_{3}, 600 \mathrm{MHZ}$

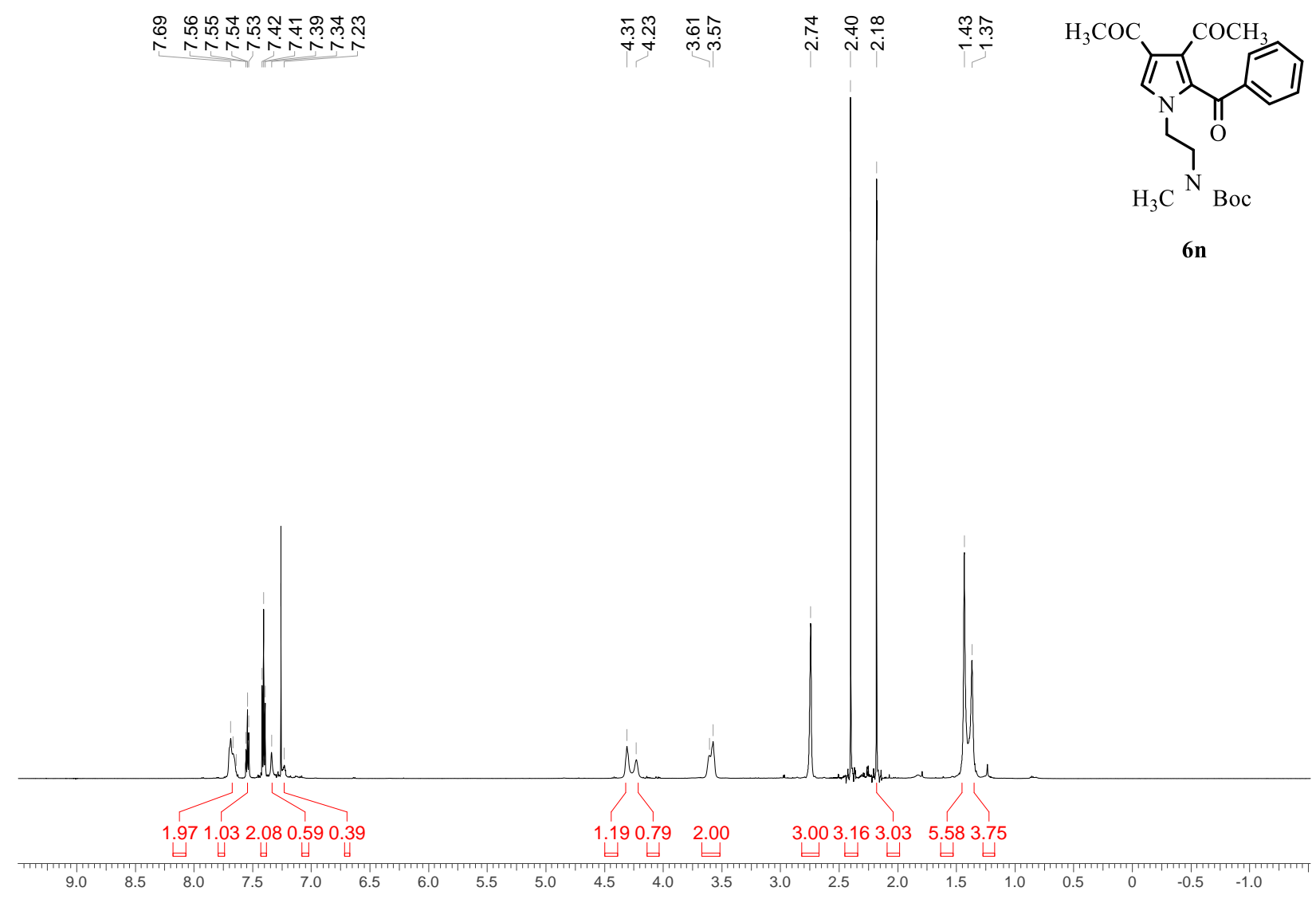

${ }^{13} \mathrm{C}$ NMR spectrum, $\mathrm{CDCl}_{3}, 150 \mathrm{MHz}$

\begin{tabular}{|c|c|}
\hline 员员 & 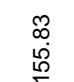 \\
\hline
\end{tabular}

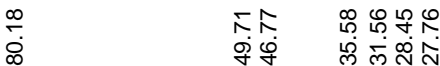

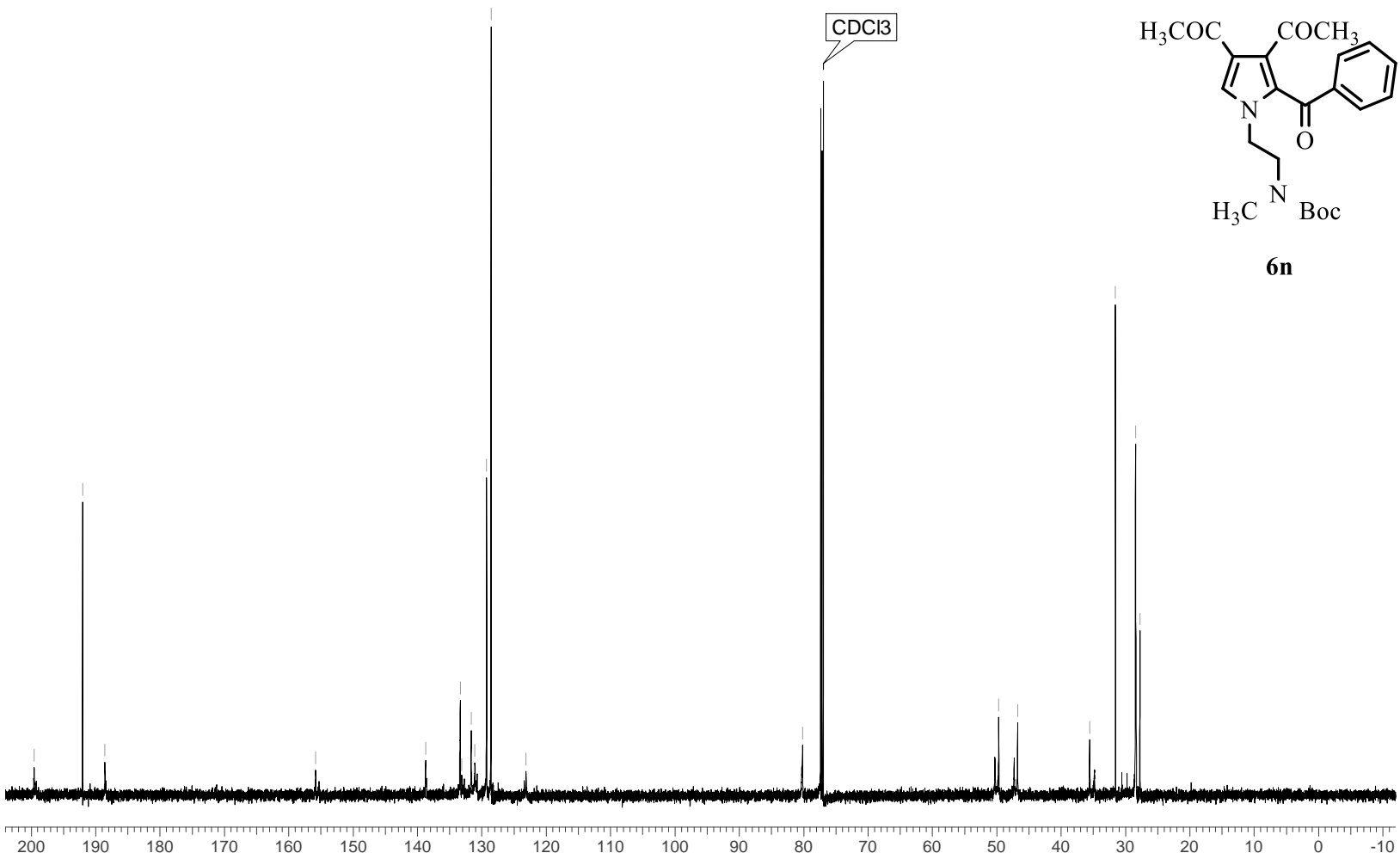


${ }^{1} \mathrm{H}$ NMR spectrum, $\mathrm{CDCl}_{3}, 600 \mathrm{MHZ}$

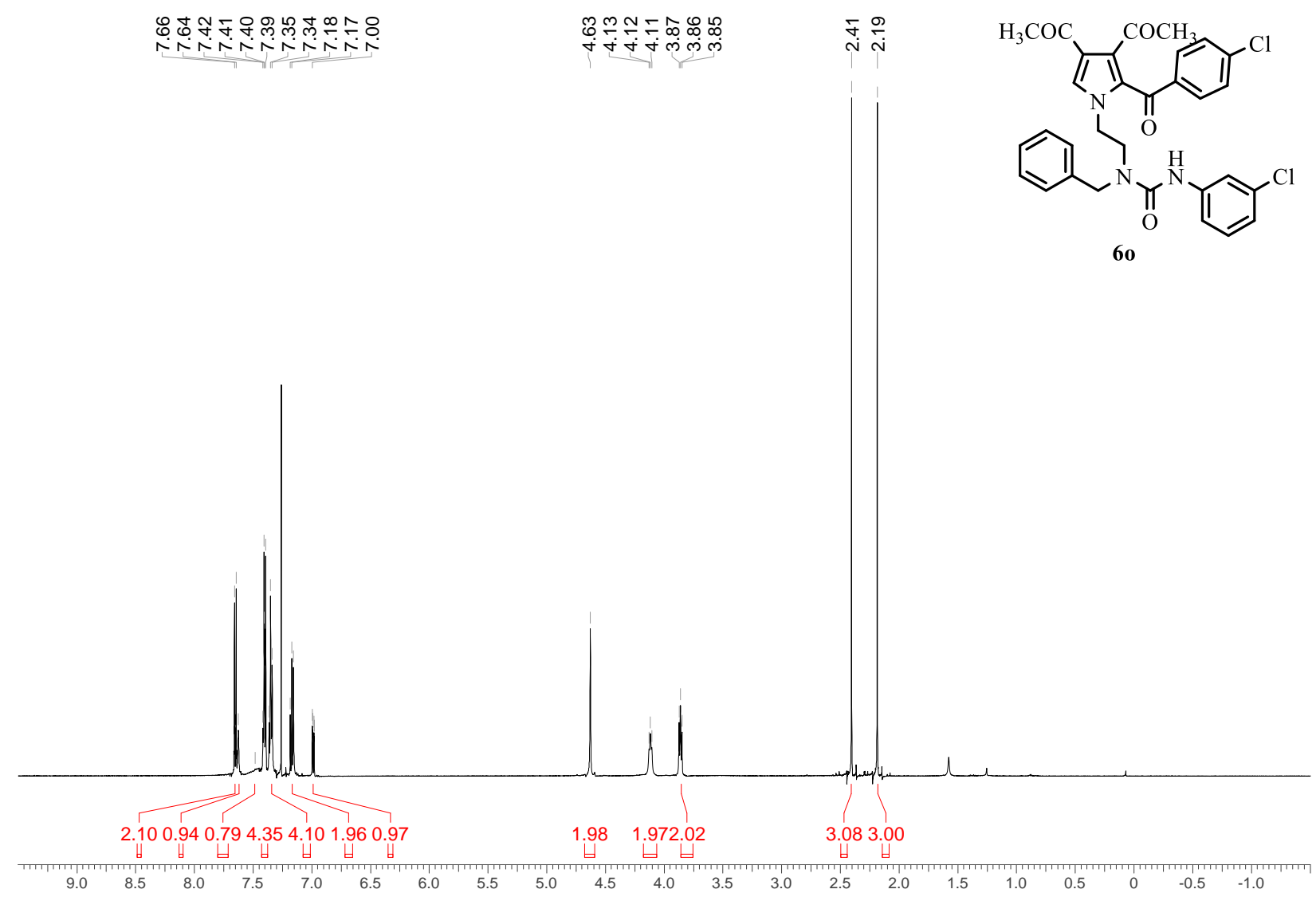

${ }^{13} \mathrm{C}$ NMR spectrum, $\mathrm{CDCl}_{3}, 150 \mathrm{MHz}$

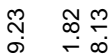

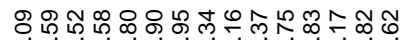

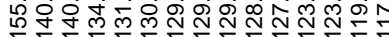

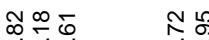

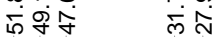<smiles>CC(=O)c1cn(CCN(Cc2ccccc2)C(=O)Nc2cccc(Cl)c2)c(C(=O)c2ccc(Cl)cc2)c1C(=O)O</smiles> 
${ }^{1} \mathrm{H}$ NMR spectrum, $\mathrm{CDCl}_{3}, 600 \mathrm{MHZ}$

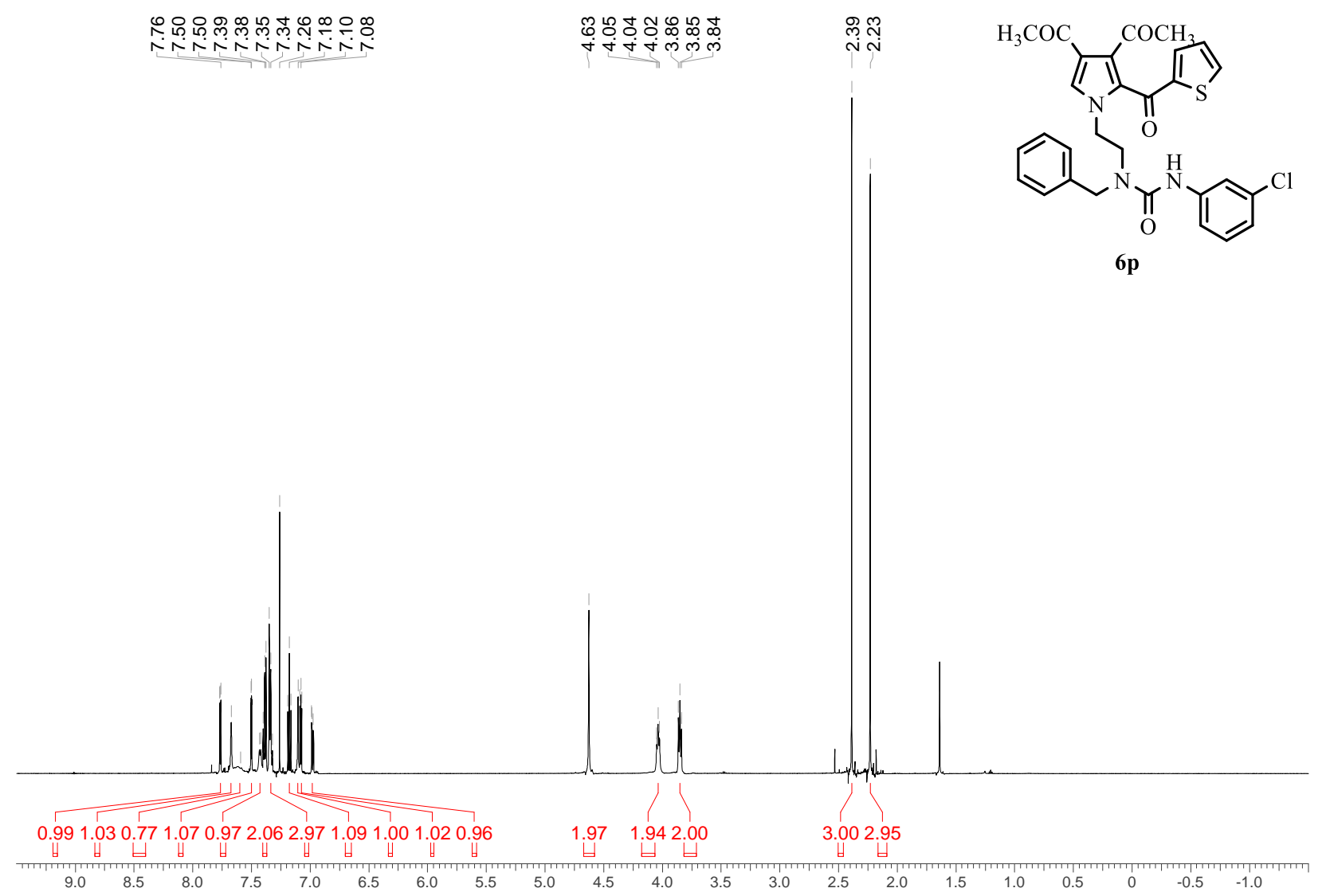

${ }^{13} \mathrm{C}$ NMR spectrum, $\mathrm{CDCl}_{3}, 150 \mathrm{MHz}$

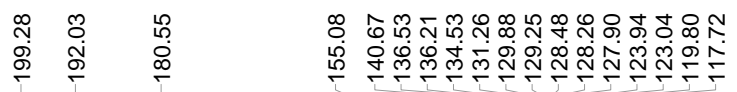

$\mathrm{CDCl} 3$

in

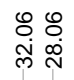

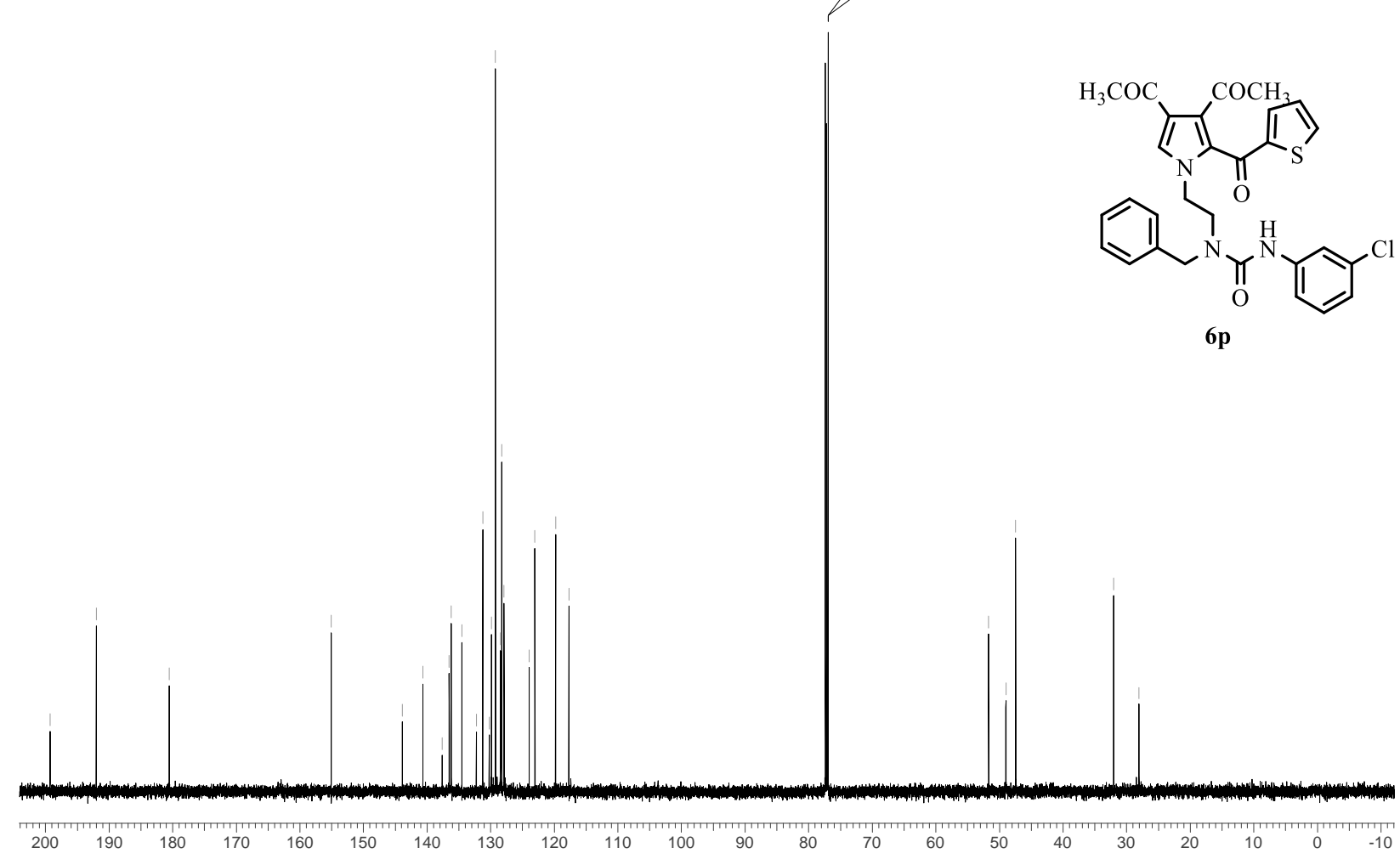


${ }^{1} \mathrm{H}$ NMR spectrum, $\mathrm{C}_{6} \mathrm{D}_{6}, 600 \mathrm{MHZ}$

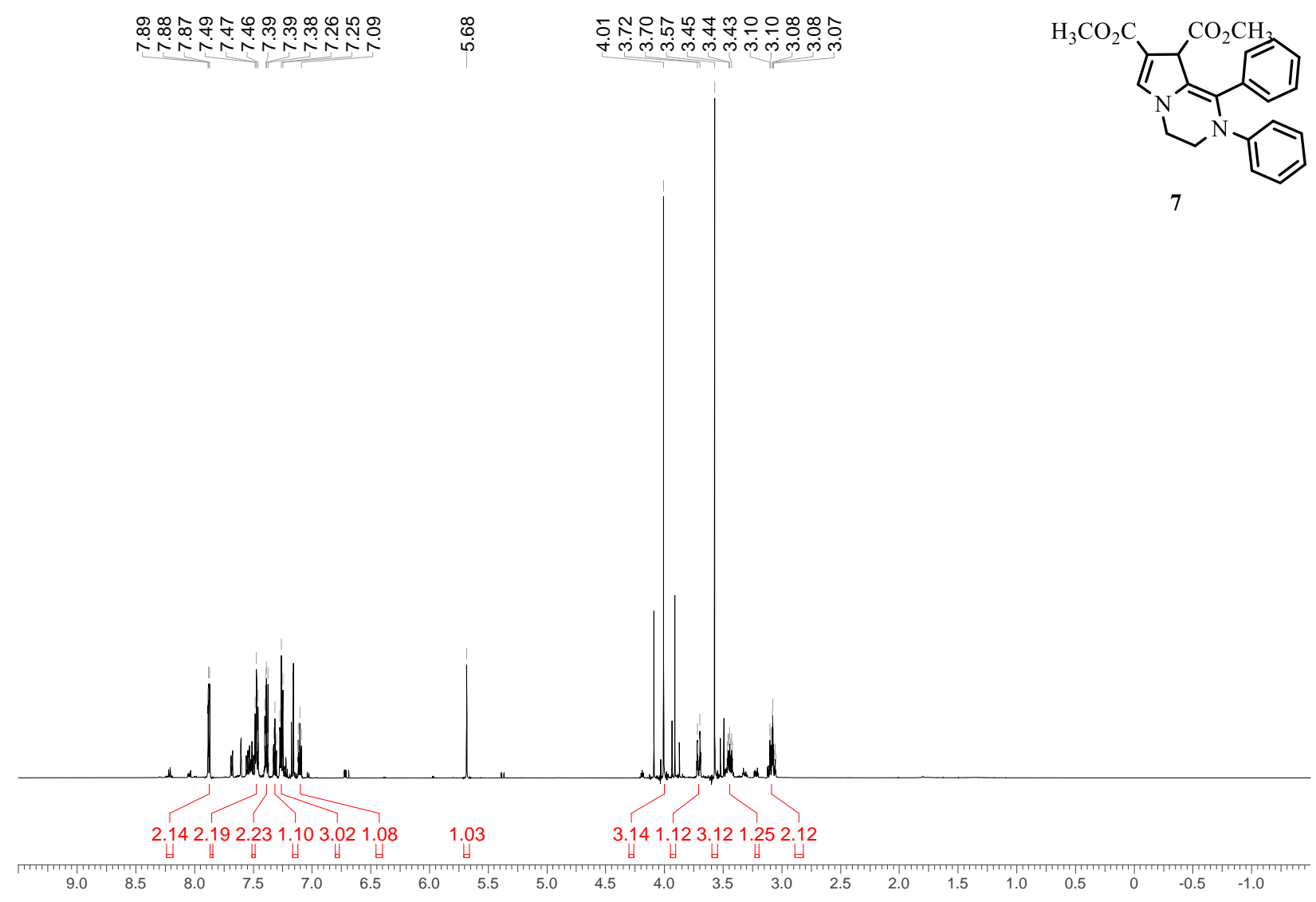

${ }^{13} \mathrm{C}$ NMR spectrum, $\mathrm{C}_{6} \mathrm{D}_{6}, 150 \mathrm{MHz}$

C6D6

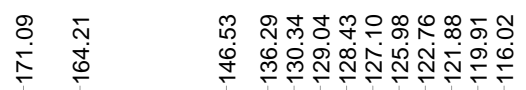

은

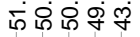

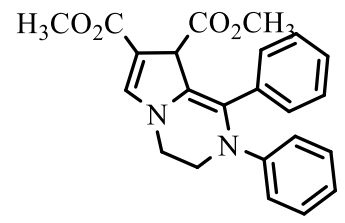

7

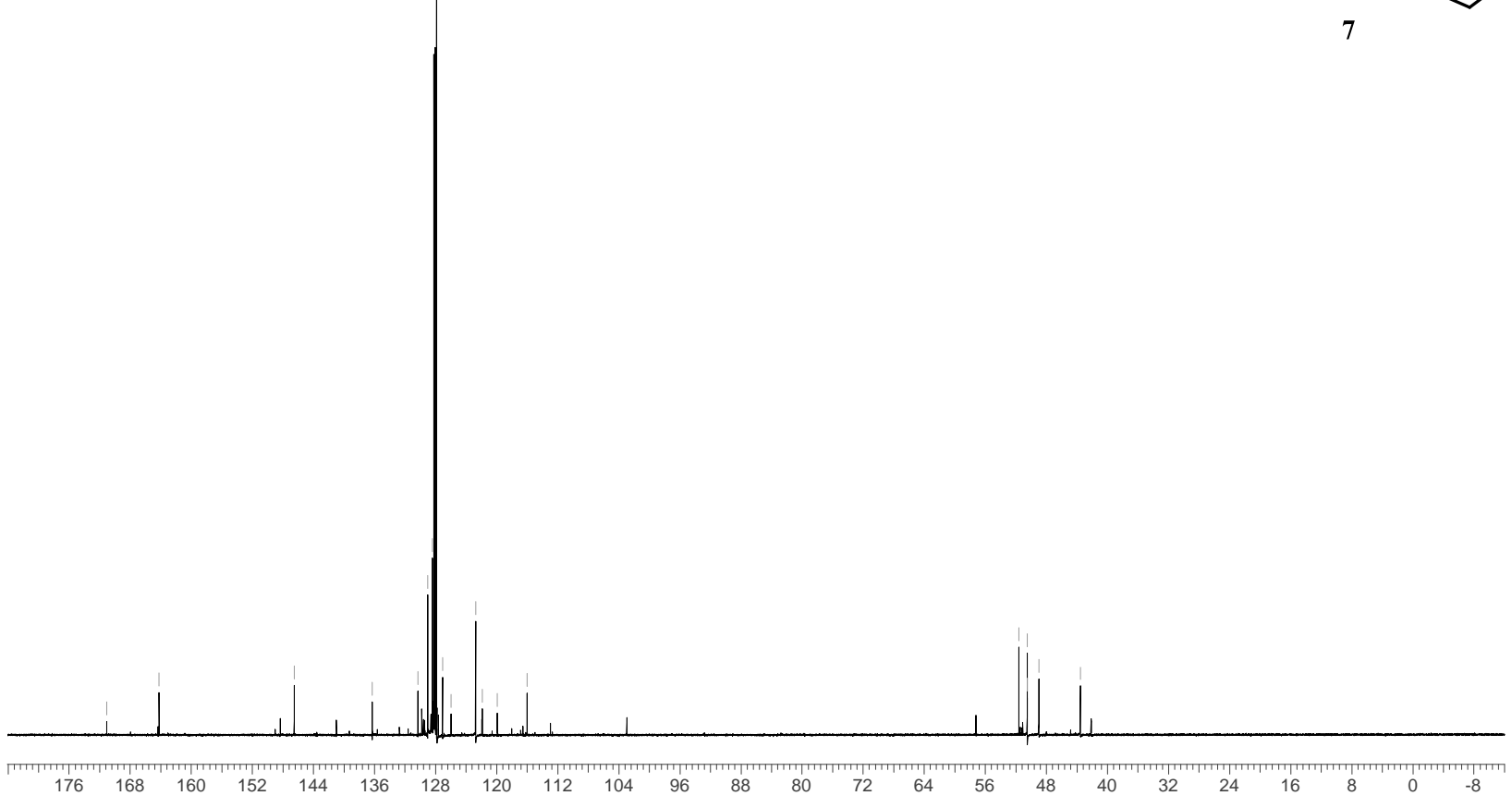

